\title{
Untersuchungen zur Variabilität und Kausalität des potentiellen Nitrataustrages beim Anbau von Zea mays in Deutschland
}

\author{
Dissertation \\ zur Erlangung des Doktorgrades \\ der Fakultät für Agrarwissenschaften \\ der Georg-August-Universität Göttingen
}

\author{
vorgelegt von \\ Thorsten Schiermann \\ geboren in Steinfurt-Borghorst
}

Göttingen, September 2004 
D 7

1. Referent: Prof. Dr. J. Isselstein

2. Korreferent: Prof. Dr. Ir. H. Van den Weghe

Tag der mündlichen Prüfung: 18.11.2004 


\section{Inhaltsverzeichnis}

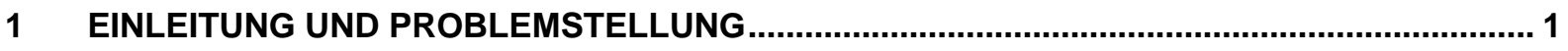

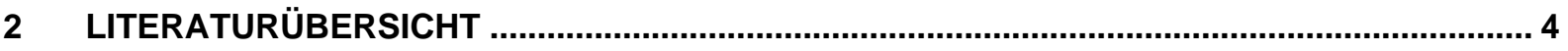

2.1 NITRATAUSTRAGSPROBLEMATIK BEIM ANBAU VON MAIS ...................................................... 4

2.2 MABNAHMEN ZUR MINDERUNG VON NITRATAUSTRÄGEN IM MAISANBAU ................................. 5

2.2.1 Düngung und Düngemittelapplikation im Maisanbau .................................................. 5

2.2.2 Acker- und pflanzenbauliche Maßnahmen zur Minderung von N-Austrägen................... 11

2.3 METHODEN ZUR ERFASSUNG DER NitRATAUSTRAgSGEFÄHRDUNG BEIM MAISANBAU................. 14

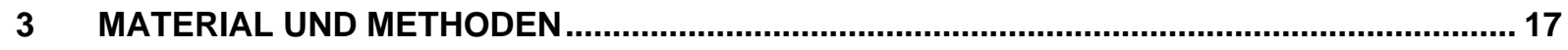

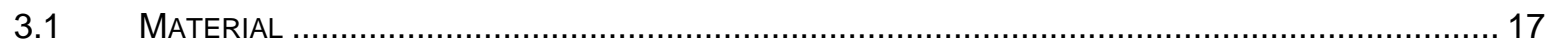

3.1.1 Daten aus der Wasserschutzgebietsberatung (Datenkategorie 1) .................................. 17

3.1.2 Daten aus Erhebungen (Datenkategorie 2) ............................................................. 20

3.1.3 Daten aus Exaktversuchen (Datenkategorie 3) ...................................................... 23

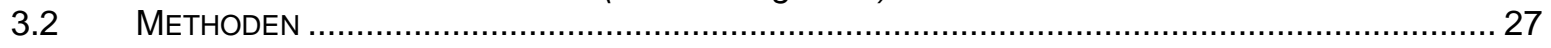

$4 \quad$ ERGEBNISSE

4.1 DATEN AUS DER WASSERSCHUTZGEBIETSBERATUNG (DATENKATEGORIE 1)........................... 32

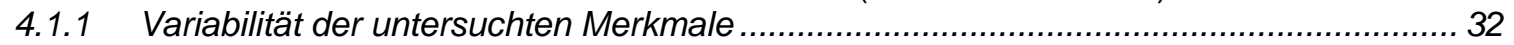

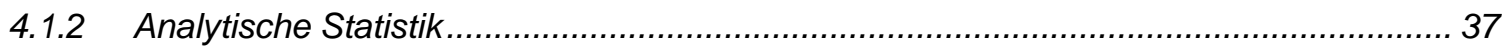

4.2 AUSWERTUNG DER DATEN AUS ERHEBUNGEN (DATENKATEGORIE 2) .................................... 51

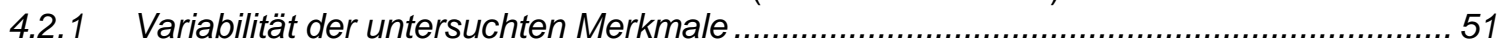

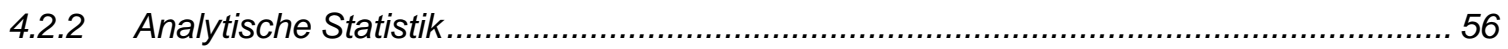

4.3 AUSWERTUNG DER DATEN AUS EXAKTVERSUCHEN (DATENKATEGORIE 3) ............................. 70

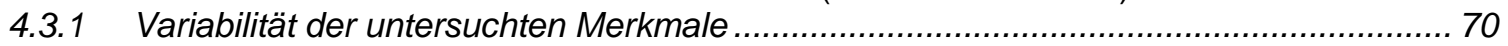

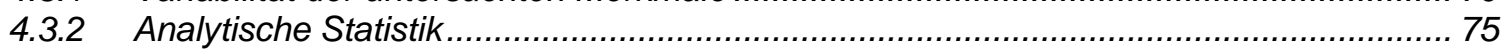

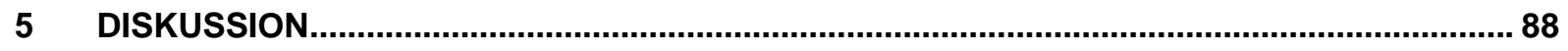

5.1 EINFLUSS DER DÜNGERMENGE AUF DEN HERBSt N MIN $^{-G E H A L T ~ I M ~ S T A N D O R T K O N T E X T ~ . . . . . . . . . . . .88 ~}$

5.2 EINFLUSS DES FLÄCHENBILANZSALDOS AUF DEN HERBST N MIN $_{\text {GEHALT } \ldots \ldots \ldots \ldots \ldots \ldots \ldots \ldots \ldots \ldots . . \ldots \ldots}$

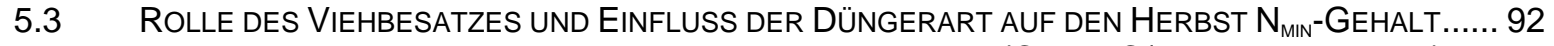

5.4 EINFLUSS MinERALISATIONSRELEVANTER BOdENMERKMALE $\left(\mathrm{C}_{\mathrm{T}}, \mathrm{N}_{\mathrm{T}}, \mathrm{C} / \mathrm{N}\right.$, FRÜHJAHRS $\left.\mathrm{N}_{\text {MIN }}\right)$ AUF

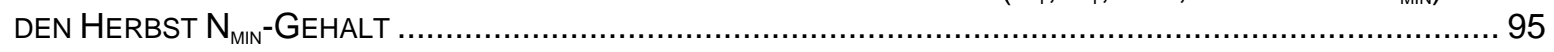

5.5 EINFLUSS DER FRUCHTFOLGEGESTALTUNG VOR DEM MAISANBAU AUF DEN FRÜHJAHRS UND

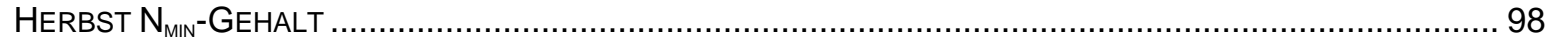

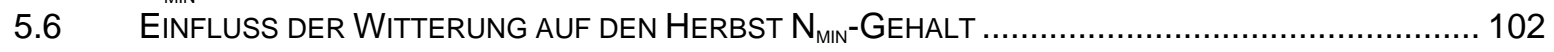

5.7 VERGLEICH DER AUSSAGEKRAFT VON ERHEBUNGSDATEN MIT ERKENNTNISSEN AUS

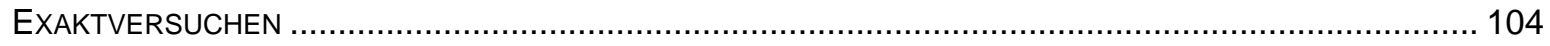

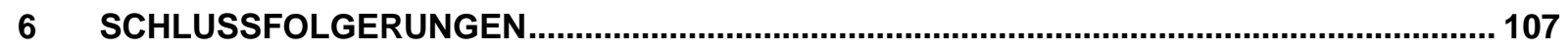

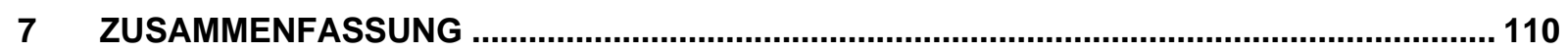

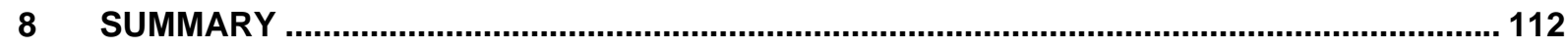

9 LITERATURVERZEICHNIS

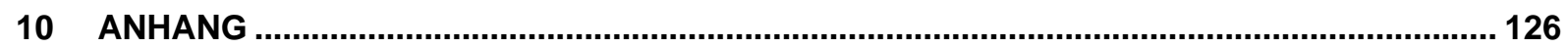




\section{Abbildungsverzeichnis}

Abb. 1: Übersicht zur räumlichen Verteilung der Daten aus den Wasserschutzgebieten. ................... 18

Abb. 2: Übersicht zur räumlichen Verteilung der Daten aus den Erhebungen. .................................. 21

Abb. 3: Lage der Exaktversuchstandorte in Deutschland ............................................................ 26

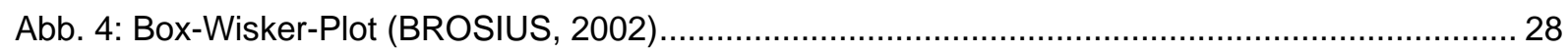

Abb. 5: Häufigkeitsverteilung der Herbst $\mathrm{N}_{\min }$-Gehalte (0-90cm / Datenkategorie 1) .......................... 32

Abb. 6: Häufigkeitsverteilung der Frühjahrs $\mathrm{N}_{\min }$-Gehalte $(0-90 \mathrm{~cm} /$ Datenkategorie 1) ...................... 33

Abb. 7: Häufigkeitsverteilung der aufgewendeten mineralischen und organischen Düngermenge in $\mathrm{kg}$

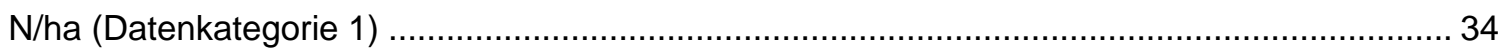

Abb. 8: Häufigkeitsverteilung der geschätzten N-Salden (kg N/ha / Datenkategorie 1) ..................... 34

Abb. 9: Häufigkeitsverteilung der Silo- und Körnermaisflächen nach gruppierten Bodenarten

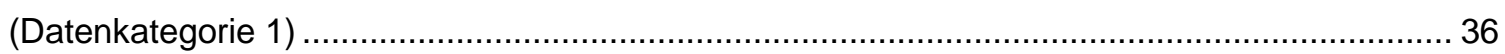

Abb. 10: Häufigkeitsverteilung der C/N-Verhältnisse (Datenkategorie 1) ......................................... 36

Abb. 11: Herbst $\mathrm{N}_{\min }$-Gehalte im Verhältnis zur ausgebrachten mineralischen und organischen Düngermenge (Korrelation nach Pearson / Datenkategorie 1) ............................................ 37

Abb. 12: Herbst $\mathrm{N}_{\min }$-Gehalte im Verhältnis zur ausgebrachten mineralischen Düngermenge ............ 39

Abb. 13: Mittlere ausgebrachte mineralische Düngermenge ( $\mathrm{kg} \mathrm{N} / \mathrm{ha})$ in Abhängigkeit vom Viehbesatz der Betriebe (< oder > 2 GV/ha / Datenkategorie 1) ............................................................. 40

Abb. 14: Herbst $\mathrm{N}_{\min }$-Gehalte im Verhältnis zur ausgebrachten organischen Düngermenge............... 40

Abb. 15: Ausgebrachte organische Düngermenge ( $\mathrm{kg} \mathrm{N} / \mathrm{ha}$ ) in Abhängigkeit vom Viehbesatz der

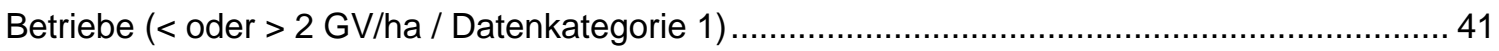

Abb. 16: Herbst $\mathrm{N}_{\text {min }}$-Gehalte von Silo- und Körnermaisflächen in Abhängigkeit vom N-Saldo (Korrelation nach Pearson / Datenkategorie 1)

Abb. 17: Herbst $N_{\min }$ Gehalte (kg N/ha) je nach Bodenart (Reichsbodenschätzung / Datenkategorie 1)

Abb. 18: Herbst $\mathrm{N}_{\min }$-Gehalte von Silo- und Körnermaisflächen in Abhängigkeit vom C/N-Verhältnis des Bodens (Korrelation nach Pearson / Datenkategorie 1)

Abb. 19: Herbst $\mathrm{N}_{\min }$-Gehalte von Silo- und Körnermaisflächen in Abhängigkeit vom Gesamt-N Gehalt des Bodens (Korrelation nach Pearson / Datenkategorie 1)

Abb. 20: Herbst $\mathrm{N}_{\min }$-Gehalte von Silo- und Körnermaisflächen in Abhängigkeit vom $\mathrm{C}_{\mathrm{t}}$-Gehalt des Bodens (Korrelation nach Pearson / Datenkategorie 1)

Abb. 21: Verteilung der Frühjahrs $\mathrm{N}_{\min }$-Gehalte nach dem Anbau von Zwischenfrüchten zur Hauptfrucht Mais (links/Probenahmetermin vor Mai), sowie Verteilung der Herbst $\mathrm{N}_{\min }$-Gehalte nach erfolgtem Maisanbau (rechts), jeweils in $\mathrm{kg} \mathrm{N} / \mathrm{ha}$ in 0-90 cm Bodentiefe (Datenkategorie 1)

Abb. 22: Verteilung der Frühjahrs $\mathrm{N}_{\text {min }}$-Gehalte zur Hauptfrucht Mais (links/Probenahmetermin vor Mai) sowie Verteilung der Herbst $\mathrm{N}_{\min }$-Gehalte nach erfolgtem Maisanbau (rechts), jeweils in $\mathrm{kg}$ $\mathrm{N} / \mathrm{ha}$ in 0-90cm Bodentiefe, in Abhängigkeit von der Art der Vorfrucht (Datenkategorie 1) 
Abb. 23: Häufigkeitsverteilung der Herbst $\mathrm{N}_{\min }$-Gehalte $(0-90 \mathrm{~cm})$ nach der Ernte und am Vegetationsende (Datenkategorie 2) 51

Abb. 24: Häufigkeitsverteilung der Frühjahrs $\mathrm{N}_{\min }$-Gehalte (0-90 cm / Datenkategorie 2)..... 52

Abb. 25: Häufigkeitsverteilung der aufgewendeten mineralischen und organischen Düngermenge in $\mathrm{kg}$ N/ha (Datenkategorie 2) 53

Abb. 26: Häufigkeitsverteilung der N-Salden (kg N/ha / Datenkategorie 2) .................................5 53

Abb. 27: Häufigkeitsverteilung der Viehbesatzdichten (GV/ha / Datenkategorie2) ..........................5 54

Abb. 28: Häufigkeitsverteilung der Silo- und Körnermaisflächen nach gruppierten Bodenarten

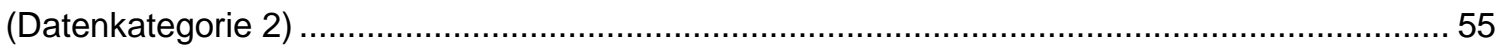

Abb. 29: Häufigkeitsverteilung der C/N-Verhältnisse (über alle Bodenarten / Datenkategorie 2) ........56

Abb. 30: Beziehung zwischen dem Herbst $\mathrm{N}_{\min }$-Gehalt (Vegetationsende) und den einzelnen Komponenten der Faktorenanalyse (partielle Korrelation / Datenkategorie 2) 58

Abb. 31: Herbst $\mathrm{N}_{\min }$-Gehalt (Vegetationsende) im Verhältnis zur ausgebrachten mineralischen und organischen Düngermenge (Korrelation nach Pearson / Datenkategorie 2)

Abb. 32: Herbst $\mathrm{N}_{\min }$-Gehalte (Vegetationsende) im Verhältnis zur ausgebrachten mineralischen Düngermenge (Korrelation nach Pearson / Datenkategorie 2)

Abb. 33: Herbst $\mathrm{N}_{\min }$-Gehalte (Vegetationsende) im Verhältnis zur ausgebrachten organischen Düngermenge (Korrelation nach Pearson / Datenkategorie 2)

Abb. 34: Herbst $\mathrm{N}_{\min }$-Gehalte (Vegetationsende) im Verhältnis zum N-Saldo (Korrelation nach Pearson / Datenkategorie2)

Abb. 35: Herbst $\mathrm{N}_{\min }$-Mengen (kg N/ha / Vegetationsende) je nach Bodenartgruppe (Datenkategorie 2)

Abb. 36: Herbst $\mathrm{N}_{\min }$-Gehalte (Vegetationsende) im Verhältnis zum C/N-Verhältnis des Bodens (Korrelation nach Pearson / Datenkategorie 2)

Abb. 37: Herbst $\mathrm{N}_{\min }$-Gehalte (Vegetationsende) im Verhältnis zum Gesamt-N Gehalt des Bodens (Korrelation nach Pearson / Datenkategorie 2)

Abb. 38: Herbst $\mathrm{N}_{\min }$-Gehalt (Vegetationsende) im Verhältnis zum $\mathrm{C}_{\mathrm{t}}$-Gehalt des Bodens (Korrelation nach Pearson / Datenkategorie 2)

Abb. 39: Herbst $\mathrm{N}_{\min }$-Gehalt (Vegetationsende) im Verhältnis zur Ackerzahl (Korrelation nach Pearson / Datenkategorie 2) 66

Abb. 40: Verteilung der Frühjahrs und Herbst $\mathrm{N}_{\text {min }}$-Gehalte (kg N/ha / Vegetationsende) in Abhängigkeit von der „Brache“ oder dem Einsatz einer „Nicht Leguminosen“ Zwischenfrucht vor dem Maisanbau (Datenkategorie 2)

Abb. 41: Verteilung der Frühjahrs und Herbst $\mathrm{N}_{\text {min }}$-Gehalte ( $\mathrm{kg} \mathrm{N} / \mathrm{ha} /$ Vegetationsende) in Abhängigkeit von der Vorfrucht vor dem Maisanbau (Datenkategorie 2).

Abb. 42: Häufigkeitsverteilung der Herbst $\mathrm{N}_{\text {min }}$-Gehalte $(0-90 \mathrm{~cm})$ nach der Ernte (Datenkategorie 3) 70

Abb. 43: Häufigkeitsverteilung der Frühjahrs $\mathrm{N}_{\text {min }}$-Gehalte $(0-90 \mathrm{~cm} /$ Datenkategorie 3). 71

Abb. 44: Häufigkeitsverteilung der aufgewendeten Düngermenge (mineralisch und organisch) in $\mathrm{kg} / \mathrm{ha}$ auf den gedüngten Flächen (Datenkategorie 3)

Abb. 45: Häufigkeitsverteilung der N-Salden bei den Flächen mit und ohne Düngung (Datenkategorie 3). 
Abb. 46: Häufigkeitsverteilung der Flächen nach gruppierter Bodenart (Datenkategorie 3) 74

Abb. 47: Häufigkeitsverteilung der C/N-Verhältnisse je nach Düngung (Datenkategorie 3)

Abb. 48: Beziehung zwischen dem Herbst $\mathrm{N}_{\min }$-Gehalt und der Komponente 1 der Faktorenanalyse (ohne Düngung / Korrelation nach Pearson / Datenkategorie 3)

Abb. 49: Beziehung zwischen dem Herbst $\mathrm{N}_{\min }$-Gehalt und den einzelnen Komponenten der Faktorenanalyse (mit Düngung / Korrelation nach Pearson / Datenkategorie 3)

Abb. 50: Herbst $\mathrm{N}_{\text {min }}$-Gehalt im Verhältnis zur ausgebrachten mineralischen und organischen Düngermenge (Korrelation nach Pearson / Datenkategorie 3)

Abb. 51: Herbst $\mathrm{N}_{\text {min }}$-Gehalte im Verhältnis zur ausgebrachten mineralischen Düngermenge (Korrelation nach Pearson / Datenkategorie 3)

Abb. 52: Herbst $\mathrm{N}_{\min }$-Gehalte im Verhältnis zur ausgebrachten organischen Düngermenge (Korrelation nach Pearson / Datenkategorie 3)

Abb. 53: Herbst $\mathrm{N}_{\min }$-Gehalte versus $\mathrm{N}$-Saldo bei den nicht gedüngten und gedüngten Flächen (Korrelation nach Pearson / Datenkategorie 3) 80

Abb. 54: Herbst $\mathrm{N}_{\min }$-Mengen je nach Bodenartgruppe (Datenkategorie 3)

Abb. 55: Herbst $\mathrm{N}_{\min }$-Gehalte in Abhängigkeit vom $\mathrm{C} / \mathrm{N}-$ Verhältnis des Bodens (Korrelation nach Pearson / Datenkategorie 3)

Abb. 56: Herbst $\mathrm{N}_{\min }$-Gehalte in Abhängigkeit vom Gesamt-N Gehalt des Bodens (Korrelation nach Pearson / Datenkategorie 3).

Abb. 57: Herbst $\mathrm{N}_{\min }$-Gehalte in Abhängigkeit vom $\mathrm{C}_{\mathrm{t}}$-Gehalt des Bodens (Korrelation nach Pearson / Datenkategorie 3)

Abb. 58: Herbst $\mathrm{N}_{\min }$-Gehalte in Abhängigkeit von der Ackerzahl (Korrelation nach Pearson / Datenkategorie 3)

Abb. 59: Verteilung der Herbst $\mathrm{N}_{\min }$-Gehalte (kg N/ha) in Abhängigkeit von der „Brache“ oder dem Einsatz einer „Nicht Leguminosen“ Zwischenfrucht vor dem Maisanbau (Datenkategorie 3)...... 85

Abb. 60: Verteilung der Herbst $\mathrm{N}_{\text {min }}$-Gehalte in Abhängigkeit von der Vorfrucht vor dem Maisanbau (Datenkategorie 3) 


\section{$\underline{\text { Tabellenverzeichnis }}$}

Tab. 1: Zusammensetzung der Daten aus den Wasserschutzgebieten nach der Randomisation (Datenkategorie 1)

Tab. 2: Zusammensetzung der Daten von den Erhebungsflächen nach der Randomisation

(Datenkategorie 2) 22

Tab. 3: Beschreibung der Daten aus Exaktversuchen.

Tab. 4: Zusammensetzung der Daten aus den Exaktversuchen nach der Randomisation (Datenkategorie 3)

Tab. 5: Mittelwert und mittlerer Rang (Kruskal-Wallis Test) der Herbst $\mathrm{N}_{\min }-$ Mengen und der $\mathrm{N}$ -

Düngung (Datenkategorie 1)

Tab. 6: Korrelation (Pearson) zwischen N-Düngermenge (mineralisch/organisch) und dem Herbst $\mathrm{N}_{\text {min }}{ }^{-}$ Gehalt (0-90cm / Datenkategorie 1) .

Tab. 7: Korrelation (Pearson) zwischen dem N-Saldo und dem Herbst $\mathrm{N}_{\min }-$ Gehalt $(0-90 \mathrm{~cm} /$

Datenkategorie 1)

Tab. 8: Charakteristika von Flächen mit besonders tiefen und auffallend hohen Herbst $\mathrm{N}_{\min }$-Gehalten

(Datenkategorie 1)

Tab. 9: Korrelation (Pearson) zwischen dem Frühjahrs und dem nachfolgenden Herbst $\mathrm{N}_{\min }$-Gehalt (0$90 \mathrm{~cm} /$ Datenkategorie 1)

Tab. 10: Korrelation (Kendall) zwischen der Witterung (Temperatur und Niederschlag) und dem Herbst $\mathrm{N}_{\text {min }}$-Gehalt (0-90cm / Datenkategorie 1)..... 50

Tab. 11: Eigenwerte und erklärte Varianzen der Faktorenanalyse (Datenkategorie 2) .......................56

Tab. 12: Rotierte Komponentenmatrix mit der entsprechenden Faktorladung (Datenkategorie 2) ...... 57

Tab. 13: Mittelwert und mittlerer Rang (Kruskal-Wallis Test) der Herbst $\mathrm{N}_{\min }$-Mengen

(Vegetationsende) und der N-Düngung (Datenkategorie 2)

Tab. 14: Korrelation (Pearson) zwischen der N-Düngermenge (mineralisch/organisch) und dem Herbst $\mathrm{N}_{\min }$-Gehalt (Vegetationsende / 0-90cm / Datenkategorie 2)..... 60

Tab. 15: Korrelation (Pearson) zwischen dem Frühjahrs und dem nachfolgenden Herbst $\mathrm{N}_{\min }$-Gehalt (0-90 cm / Datenkategorie 2) . 66

Tab. 16: Multiple Regression mit dem Herbst $\mathrm{N}_{\min }$-Gehalt (Vegetationsende) als abhängige Variable (Regressor / Datenkategorie 2)

Tab. 17: Korrelation (Kendall) zwischen der Witterung (Temperatur und Niederschlag) und dem Herbst $\mathrm{N}_{\min }$-Gehalt (0-90 cm / Vegetationsende / Datenkategorie 2)...

Tab. 18: Eigenwert und erklärte Varianz der Faktorenanalyse (ohne Düngung / Datenkategorie 3) ... 75

Tab. 19: Komponentenmatrix mit der entsprechenden Faktorladung (ohne Düngung / Datenkategorie 3)

Tab. 20: Eigenwerte und erklärte Varianzen der Faktorenanalyse (mit Düngung / Datenkategorie 3) 76

Tab. 21: Rotierte Komponentenmatrix mit der entsprechenden Faktorladung (mit Düngung / Datenkategorie 3)

Tab. 22: Korrelation (Pearson) zwischen der N-Düngermenge (mineralisch/organisch) und dem Herbst $\mathrm{N}_{\min }$-Gehalt (0-90cm / Datenkategorie 3)... 
Tab. 23: Korrelation (Pearson) zwischen dem Frühjahrs und dem nachfolgendem Herbst $\mathrm{N}_{\min }-$ Gehalt (0-90cm / Datenkategorie 3) . 83

Tab. 24: Multiple Regression mit dem Herbst $\mathrm{N}_{\min }$-Gehalt als abhängige Variable (Regressor /

Datenkategorie 3) 85

Tab. 25: Korrelation (Kendall) zwischen der Witterung (Temperatur und Niederschlag) und dem Herbst $\mathrm{N}_{\text {min }}$-Gehalt (0-90cm / Datenkategorie 3). 87 


\section{Anhangsverzeichnis}

Anhang 1: Häufigkeitsverteilung der Herbst $\mathrm{N}_{\min }-$ Gehalte $(\mathrm{kg} \mathrm{N} / \mathrm{ha} / 0-90 \mathrm{~cm})$ in den

Haupterhebungsjahren (1996-1999 / Datenkategorie 1).

Anhang 2: Häufigkeitsverteilung der Frühjahrs $\mathrm{N}_{\text {min }}$-Gehalte $(0-90 \mathrm{~cm})$ differenziert danach ob sie vor dem 1. Mai oder ab dem 1 Mai gezogen wurden (Datenkategorie 1) ......

Anhang 3: Häufigkeitsverteilung der Silo- und Körnermaiserträge (Datenkategorie 1) .....

Anhang 4: Häufigkeitsverteilung der Vorfrüchte, getrennt nach den Hauptfrüchten Silo- und Körnermais (Datenkategorie 1)

Anhang 5: Häufigkeitsverteilung der Zwischenfrüchte, getrennt nach den Hauptfrüchten Silo- und Körnermais (Datenkategorie 1)...

Anhang 6: Gruppierung der Bodenarten nach leicht, mittel und schwer aus Angaben nach DIN4220 und der Reichsbodenschätzung (LANZRATH und GOLDBACH, 2001) .

Anhang 7: Durchschnittlicher Temperaturverlauf $\left({ }^{\circ} \mathrm{C}\right)$ in den Haupterhebungsjahren (Datenkategorie 1)

Anhang 8: Durchschnittlicher Niederschlag ( $\mathrm{mm})$ in den Haupterhebungsjahren (Datenkategorie 1) 129

Anhang 9: Durchschnittliche potentielle Evapotranspiration ( $\mathrm{mm}$ ) nach HAUDE in den

Haupterhebungsjahren (Datenkategorie 1)

Anhang 10: Häufigkeitsverteilung der aufgewendeten Düngermenge (mineralisch und organisch) in $\mathrm{kg}$ N/ha. Das erste Diagramm (oben links) beschreibt alle Daten der Datenkategorie 2. Das Zweite (oben rechts), das Dritte (unten links) und das vierte Diagramm (unten rechts) beschreiben die Häufigkeitsverteilungen der Düngermenge je nach Versuchsansteller. (Die Versuchsansteller werden in der X-Achsen Bezeichnung genannt / Datenkategorie 2).

Anhang 11: Häufigkeitsverteilung der Silomaiserträge (links Frischmasse (FM)/ rechts Trockenmasse

(TM) / Datenkategorie 2)

Anhang 12: Häufigkeitsverteilung der Körnermaiserträge (links Frischmasse (FM)/ rechts

Trockenmasse (TM) / Datenkategorie 2).

Anhang 13: Häufigkeitsverteilung der Vorfrüchte, getrennt nach den Hauptfrüchten Silo- und Körnermais (Datenkategorie 2)...

Anhang 14: Häufigkeitsverteilung der Zwischenfrüchte, getrennt nach Silo- und Körnermais

(Datenkategorie 2)

Anhang 15: Häufigkeitsverteilung der Frühjahrs $\mathrm{N}_{\min }$-Gehalte $(0-90 \mathrm{~cm})$ differenziert danach ob sie vor

dem 1. Mai oder ab dem 1 Mai gezogen wurden (Datenkategorie 3) ..................................... 134

Anhang 16: Häufigkeitsverteilung der Silomaiserträge (Datenkategorie 3) ...................................... 135

Anhang 17: Häufigkeitsverteilung der Körnermaiserträge (Datenkategorie 3) ................................. 135

Anhang 18: Häufigkeitsverteilung der Vorfrüchte (Datenkategorie 3) ............................................. 136

Anhang 19: Häufigkeitsverteilung der Zwischenfrüchte (Datenkategorie 3) ...................................... 136

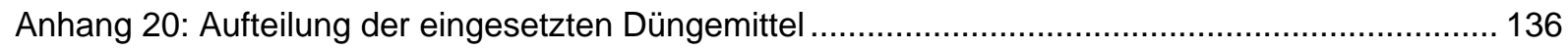




\section{Abkürzungsverzeichnis}

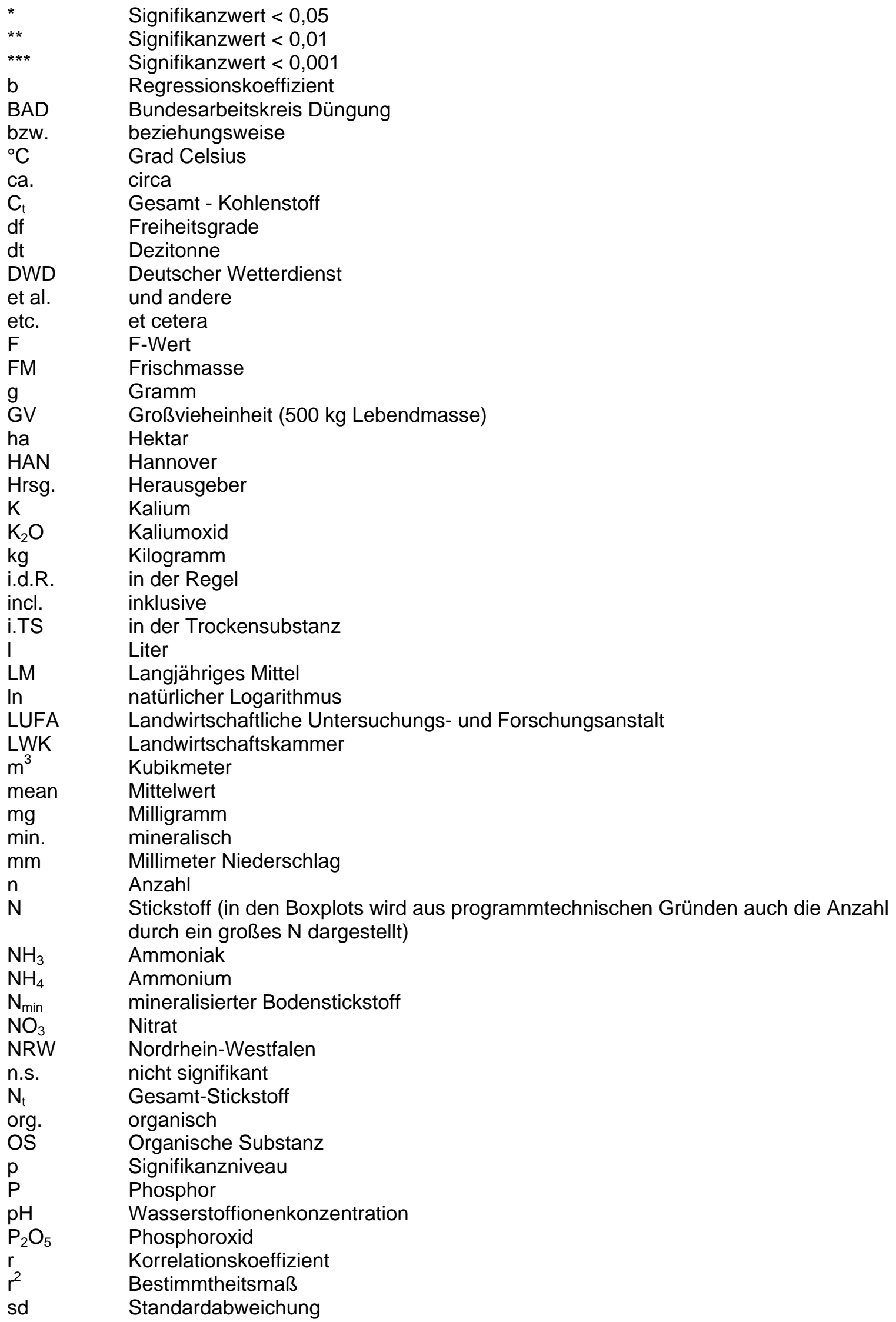




$\begin{array}{ll}\text { SF } & \text { Standardfehler } \\ \text { SH } & \text { Schleswig-Holstein } \\ \text { SPSS } & \text { Statistical Package for the Social Sciences } \\ \text { t } & \text { t-Wert } \\ \text { TM } & \text { Trockenmasse } \\ \text { TS } & \text { Trockensubstanz } \\ \text { u.a. } & \text { unter anderem } \\ \text { u.U. } & \text { unter Umständen } \\ \text { VDLUFA } & \text { Verband Deutscher Landwirtschaftlicher Untersuchungs- und Forschungsanstalten } \\ \text { vgl. } & \text { Vergleiche } \\ \text { WE } & \text { Weser-Ems } \\ \text { WL } & \text { Westfalen-Lippe } \\ \text { Z.B. } & \text { zum Beispiel } \\ \text { Zf } & \text { Zwischenfrucht } \\ \text { z.T. } & \text { zum Teil } \\ \text { Z. Zt. } & \text { zur Zeit } \\ \% & \text { Prozent }\end{array}$




\section{Einleitung und Problemstellung}

Weltweit hat sich der Mais zu einer der wichtigsten Kulturarten entwickelt. Unter den Getreidearten rangierte Mais 2002 mit 1,51 Mio. ha Anbaufläche nach Weizen und Gerste in der BRD an dritter Stelle (AGRARBERICHT, 2003).

Die Gründe für die starke Ausdehnung des Maisanbaues seit 1960 sind in der Züchtung leistungsfähiger Sorten, der Verbesserung der Produktionsverfahren sowie der betrieblichen Spezialisierung zu suchen.

Parallel zur steigenden Anbaufläche des Maises hat sich die Nitratproblematik in den landwirtschaftlich intensiv genutzten Regionen trotz umfangreicher Bemühungen eher verschärft als entspannt (HILMES et al., 1998; BISCHOFF et al., 1999). So können seit geraumer Zeit nur wenige Wasserversorger stagnierende oder gar rückläufige $\mathrm{NO}_{3}$-Konzentrationen im oberflächennahen Grundwasser beobachten (BERG et al., 1997; FREDE und DABBERT, 1999). Insbesondere die Nitratauswaschung unter Maisanbauflächen wird als hoch eingestuft (VETTER und STEFFENS, 1988; EULENSTEIN et al., 1993; STICKSEL, 1994; HEGE et al., 2001).

Seit den 80er Jahren beschäftigen sich zahlreiche Forschungsprojekte mit der Erarbeitung von Strategien zur Verminderung der Nitratauswaschung beim Maisanbau. So wurden insbesondere für landwirtschaftlich genutzte Wassergewinnungsgebiete verschiedene pflanzenbauliche Maßnahmen zur Verminderung der N-Austräge entwickelt (ANTONY et al., 2001). Produktionstechnische Maßnahmen betrafen in erster Linie das Düngesystem incl. der Bemessung von organischen und mineralischen Düngemitteln, den Anbau von Zwischenfrüchten und Untersaaten, die Anbautechnik (z.B. Reihenweite), oder auch die Art und den Zeitpunkt der Bodenbearbeitung. Viele dieser Methoden und Maßnahmen erwiesen sich auf verschiedenen Standorten als wirksam. Obwohl diese Maßnahmen für die landwirtschaftliche Praxis überwiegend verfügbar sind, sind deren Umsetzung und Wirkung in den Wasserschutzgebieten nicht immer gegeben (SCHULTHEIß et al., 1996; KRÄMER et al., 1999; MOHAUPT et al., 2000).

Ein Erklärungsansatz für diese widersprüchlichen Befunde zur Wirksamkeit produktionstechnischer Maßnahmen könnte die nicht hinreichende Beachtung von Interaktionen zwischen Standort- und Bewirtschaftungsfaktoren sein. In diesen Interaktionen liegen unter Umständen Informationen verborgen. Die Analyse dieser Interaktionen könnte den Aussagewert der Daten markant erhöhen, die Interpretation 
der kausalen Zusammenhänge verbessern und dadurch innovative Ansätze für die weiterführende Forschung ermöglichen (SCHNUG, 1985; BACKHAUS et al., 2000). Sowohl der Umfang der mit den ökologischen Zielgrößen im mutmaßlichen Zusammenhang stehenden Merkmale als auch deren vielschichtige gegenseitige Beeinflussung erfordern es, mit multivariaten statistischen Verfahren (wie z.B. Faktorenanalyse, Regressionsanalyse) zu arbeiten. Anderenfalls ist es nicht möglich, Zusammenhänge und Aussagen soweit von Wechselwirkungen zu befreien, dass sie unverfälscht zu Tage treten und verallgemeinert werden können.

Die Vereinheitlichung der Rahmenbedingungen im Versuchswesen (ceterius paribus - Prinzip) zur Aufdeckung der vornehmlich produktionstechnisch beeinflussten Haupteffekte verleitet im Zuge der Versuchsplanung zur Reduzierung bzw. Dimensionseinengung bei der Ergebnisinterpretation.

Um der Komplexität der Nitratproblematik im Maisanbau gerecht zu werden, ist es erforderlich, dass die Vielfalt der Einflussfaktoren gleichzeitig berücksichtigt wird. Das Ziel der vorliegenden Arbeit ist es, durch die Zusammenführung und gemeinsame Verrechnung einer großen Anzahl von Feldversuchen sowie von Daten, die im Rahmen der Wasserschutzgebietsberatung erhoben wurden, die Bedeutung wichtiger produktionstechnischer und standortbezogener Faktoren sowie deren Wechselwirkung für die Nitrataustragsgefahr im Maisanbau zu analysieren. Dazu wurden drei Datenkategorien gebildet. Kategorie 1 repräsentiert Daten aus der Wasserschutzgebietsberatung, die neben Messdaten auch Schätzwerte beinhaltet. Datenkategorie 2 stellt ebenfalls Praxiserhebungen dar, deren Merkmalserfassung jedoch ausschließlich auf Messdaten basiert. In die Datenkategorie 3 flossen ausschließlich wiederholte Feldversuche ein. Mit zunehmender Datenpräzision ging eine Reduktion der auswertbaren Datensätze einher. 
Folgende Fragestellungen wurden anhand der drei Datenkategorien untersucht:

- Welchen Einfluss hat die Düngermenge auf den Herbst $\mathrm{N}_{\min }$-Gehalt im Standortkontext?

- Wie wirkt sich der Flächenbilanzsaldo auf den Herbst $\mathrm{N}_{\text {min }}$-Gehalt aus?

- Beeinflusst der Viehbesatz und die Düngeart den Herbst $\mathrm{N}_{\text {min- }}$-Gehalt?

- Welchen Einfluss üben mineralisationsrelevante Bodenmerkmale $\left(C_{t}, N_{t}, C / N\right.$, Frühjahrs $\mathrm{N}_{\min }$-Gehalt) auf den Herbst $\mathrm{N}_{\text {min }}$-Gehalt aus?

- Welche Effekte hat die Fruchtfolgegestaltung vor dem Maisanbau auf den Frühjahrs und Herbst $\mathrm{N}_{\min }$-Gehalt?

- Welchen Einfluss hat die Witterung auf den Herbst $\mathrm{N}_{\min }$-Gehalt?

- Sind Erkenntnisse aus Erhebungsdaten vergleichbar mit Ergebnissen aus Exaktversuchen? 


\section{Literaturübersicht}

\subsection{Nitrataustragsproblematik beim Anbau von Mais}

Nach HAMM (1991) ist die Landwirtschaft ein Hauptverursacher der Nitratbelastung der Gewässer. Die Fruchtart Mais gilt hierbei als besonders gefährdend für das Grundwasser (BRANDHUBER und HEGE, 1991a). Dies zeigt sich durch die zumeist überdurchschnittlich hohen Nitratmengen im Boden nach der Ernte (APPEL et al., 1992, LWK-WE, 1997). Die dem Mais angelastete und auf vielen Standorten nachgewiesene Umweltbelastung des Nitrataustrages (ENGEL und MANGSTL, 1988; SIMON et al., 1988) liegt häufig an der nicht an den Bedarf angepassten organischen oder mineralischen N-Düngung (LÜTKE ENTRUP et al., 1995b). Neben der Düngung ist besonders die langsame Jugendentwicklung der Maispflanzen und die damit einhergehende zögerliche N-Aufnahme durch den Mais als problematisch anzusehen (MAIDL und FISCHBECK, 1989). Die Wurzeln erreichen im 5-6 Blattstadium erst eine Tiefe von 20-25 cm und erschließen den Reihenzwischenraum nicht hinreichend (HUGGER, 1995). Durch die einsetzende N-Mineralisation und die Düngung von Stickstoff kann dies zu sehr hohen $\mathrm{N}_{\min }$-Mengen und somit zu starken Auswaschungsverlusten führen (MAIDL, 1997; AUFHAMMER et al., 1991). Dieses spezielle Phänomen der sommerlichen Auswaschungsverluste tritt insbesondere auf flachgründigen Böden auf (STICKSEL et al., 1994a). Nach einer zögerlichen Jugendentwicklung mit geringem Bedarf setzt eine besonders intensive Phase des Massenwuchses ein, in der der Nährstoffbedarf erheblich steigt. Den höchsten Nährstoffbedarf hat der Mais zwischen dem 6-8 Blattstadium und dem Eintrocknen der Narbenfäden. In diesem Zeitraum werden rund 85\% des gesamten N-Bedarfes aufgenommen (HUGGER, 1995). In dieser Phase des intensiven Sprosswachstums bis zum Rispenschieben werden die Wurzeln im $30 \mathrm{~cm}$ Horizont stark vermehrt und einige bis zu einer Tiefe von $60 \mathrm{~cm}$ ausgebildet (HUGGER, 1995). Aber selbst nach Beginn der intensiven Stickstoffaufnahme kann es unter extremen Bedingungen zu Nitratauswaschungen im Bereich zwischen den Reihen kommen (MAIDL, 1990). Vom Rispenschieben bis zur Blüte wird das Wurzelvolumen nochmals erhöht und einige Wurzeln dringen in Tiefen von bis zu einem Meter vor. Laut WIESLER und HORST (1993) können die Wurzeln der Maispflanzen in manchen Jahren Stickstoff 
aus Bodentiefen von 120 bis $150 \mathrm{~cm}$ aufnehmen. Trotz der weiteren Wurzelausbildung kann es nach der Blüte, vor allem wegen der nachlassenden NAufnahme, zu steigenden N-Mengen im Oberboden kommen (HUGGER, 1992). Die Folge können erhöhte und damit potentiell austragsgefährdete Restnitratmengen im Boden sein. Aus diesem Grund halten ARNOLD et al. (1993) und KÖPPEN (1997) den Maisanbau in Wasserschutzgebieten für besonders kritisch. Hingegen beurteilen NITSCH (1991), GUTSER und WAGNER (2000) sowie LÜTKE ENTRUP (2002) den Mais als positive Fruchtart, da er in der Hauptbedarfsphase den mineralisierten Stickstoff des Bodens gut zu nutzen vermag.

\subsection{Maßnahmen zur Minderung von Nitratausträgen im Maisanbau}

Strategien zur Reduzierung der Nitrataustragsgefahr beim Maisanbau betreffen acker- und pflanzenbauliche Maßnahmen sowie die Anpassung der Düngung an den zeitlich und mengenmäßig ermittelten N-Bedarf des Maises (DINNES et al., 2002). Die wesentlichen Maßnahmen werden im Folgenden vorgestellt.

\subsubsection{Düngung und Düngemittelapplikation im Maisanbau}

Im Mittelpunkt vieler Forschungsarbeiten steht die Entwicklung von Verfahren zur bedarfsgerechten N-Ernährung der Pflanzen. Hierzu gehören neben der Ermittlung der dem Pflanzenbedarf angepassten Düngermenge auch Maßnahmen zur optimalen Applikation (Technik, Zeitpunkt).

\section{- Methoden der Düngebedarfsermittlung}

Im Maisanbau besteht im Gegensatz zum Getreide das Problem, dass eine Befahrbarkeit nach Reihenschluss nicht mehr gegeben ist. Deshalb spielt eine möglichst präzise Prognose der zu verabreichenden Menge an Stickstoff beim Anbau von Mais eine entscheidende Rolle. Hierfür stehen dem Landwirt verschiedene Methoden der Bemessung des N-Düngebedarfs der Maispflanze und zur Abschätzung der N-Nachlieferung des Bodens zur Verfügung. 


\section{Bilanzrechnungen}

Die einfache Bilanzierung (Düngung minus Entzug) eignet sich nur für die Quantifizierung von betrieblichen und schlagbezogenen N-Überschüssen (WERNER, 1995) und nicht zur Düngebedarfsermittlung, da sie das N-Nachlieferungspotential nicht berücksichtigt (APPEL et al., 1993). VON FISCHER $(1992,1993)$ hat ein modifiziertes Bilanzierungsverfahren vorgeschlagen, das für die Düngebedarfsermittlung verwendet werden kann. Es basiert auf Faustzahlen, die aus Versuchsergebnissen und Erhebungen im Rheinland gewonnen wurden. Daher hat das Verfahren nur regionale Gültigkeit, der Bilanzierungsansatz ist jedoch verallgemeinerbar. Berücksichtigt werden die N-Nachlieferung des Bodens, die NReserven aus Vorfrucht, Zwischenfrucht und Wirtschaftsdüngern, die zu einer Gesamt-N-Menge zusammengefasst werden. Von dieser kalkulierten Gesamt-NMenge wird der von der Ertragserwartung abhängige N-Entzug durch Mais abgezogen. Damit ist die zu ergänzende mineralische N-Düngung bestimmt. Nach Versuchen von LÜTKE ENTRUP et al. (1997a) liegen die Düngeempfehlungen bei diesem Verfahren bei geringem tatsächlichen Bedarf zu hoch und bei hohem Bedarf zu niedrig.

\section{EUF-Methode}

Ziel der EUF-Methode ist es, den mineralischen und nachlieferbaren Stickstoff im Boden $\mathrm{zu}$ erfassen und dadurch eine treffsichere Bedarfsprognose für Stickstoffdünger im Maisanbau abzugeben. Im Sommer oder Herbst wird eine Bodenprobe aus 0-30 cm Tiefe entnommen und getrocknet (KOLB, 1989). Nachfolgend wird Boden- $\mathrm{N}$ schrittweise extrahiert. In der ersten Fraktion wird der leicht verfügbare Stickstoff gewonnen; in der anschließenden zweiten Fraktion der Stickstoff, der im Frühjahr nachgeliefert werden kann. Aus dem Verhältnis (EUF- $\mathrm{N}_{\text {org- }}{ }^{-}$ Quotient) der beiden Fraktionen zueinander bekommt man Aufschluss über das Ausmaß und die Dauer der Stickstoffnachlieferung aus dem Boden (HORN, 1990a; FÜRSTENFELD und HETTERICH, 1992). Nach NEMETH (1988) ist das Nachlieferungspotential des Bodens umso geringer, je niedriger der EUF- $\mathrm{N}_{\text {org }}{ }^{-}$ Quotient ist. Auf Basis der Stickstoffnachlieferung des Bodens werden dann Düngeempfehlungen für den Mais erarbeitet (HORN, 1990b). HORN (1990b) belegt in seinen Untersuchungen, dass es bei einer N-Düngung nach EUF-Empfehlung nicht zu steigenden Restnitratgehalten nach der Ernte kommt. Die EUF-Methode ist 
laut GRÖBLINGHOFF (1992) eine Methode, für die ein sehr hoher Analyseaufwand betrieben werden muss.

\section{Nmin-Methode}

Mit der $\mathrm{N}_{\min }$-Methode werden die Menge und die Verteilung des pflanzenverfügbaren bzw. mineralischen Stickstoffs (Ammonium und Nitrat) im Boden $(0-90 \mathrm{~cm})$ gemessen (SCHARPF und WEHRMANN, 1979). Der ermittelte Wert erlaubt keine Aussage über die zu erwartende Mineralisierung. Die im Boden vorhandene $\mathrm{N}_{\text {min }^{-}}$ Menge wird durch Düngung auf einen kultur- und standortspezifischen N-Sollwert 'aufgefüllt'. Der N-Sollwert ist die N-Menge, die der Pflanze für optimale Erträge zur Verfügung stehen muss. Die Sollwerte wurden für jede Kultur durch Versuche ermittelt. Bei der klassischen $\mathrm{N}_{\text {min }}$-Methode wird die Beprobung der Fläche zwischen Anfang und Mitte April durchgeführt. Anschließend wird auf den Sollwert der jeweiligen Region (z.B. Rheinland $190 \mathrm{~kg} \mathrm{~N} / \mathrm{ha}$ ) aufgedüngt. Diese Methode hat den Nachteil, dass sie die erst später einsetzende N-Nachlieferung des Bodens nur unzureichend berücksichtigt und die Pflanzen nicht optimal mit Nährstoffen versorgt werden (LÜTKE ENTRUP et al., 1997b; BROCKERHOFF, 1994; LAURENZ, 1992).

Bei der sogenannten späten $\mathrm{N}_{\min }$-Methode werden Bodenproben zwischen Mitte Mai und Anfang Juni gezogen. Zu diesem Zeitpunkt ist die tatsächliche standort- und witterungsspezifisch mineralisierte N-Menge in der Ackerkrume am höchsten (LÜTKE ENTRUP et al., 1995a; RICHARDS et al., 1999). Je nach Standort benötigt der Mais Anfang Juni zur optimalen Ertragsbildung eine $\mathrm{N}_{\text {min }}$-Menge im Boden von 150 bis 200 $\mathrm{kg}$ pro ha (LAURENZ, 1998). Der niedrigere, als Sollwert (für Höchstertrag notwendige $\mathrm{N}$ - Menge) anzusetzende Wert gilt für stark mineralisierende Standorte. Für schwach mineralisierende Standorte sollte als Sollwert der hohe Wert eingesetzt werden. Bei der späten $\mathrm{N}_{\text {min }}$-Methode weist HEGE (1996) darauf hin, dass diese immer wieder unerklärlich hohe $\mathrm{N}_{\min }$-Gehalte liefert, weswegen seines Erachtens die klassische Methode zu bevorzugen ist.

\section{$\mathrm{CaCl}_{2}$-Norg-Methode}

Mit diesem noch in der Entwicklung befindlichen Verfahren wird der Gehalt an extrahierbarem organischen Stickstoff bestimmt. Bisher ist es trotz vieler Untersuchungen nicht gelungen, auf Basis der leicht löslichen organischen $\mathrm{N}$ Verbindungen einen eindeutigen Zusammenhang zwischen dieser Stickstofffraktion 
und dem Düngeoptimum der einzelnen Kulturen nachzuweisen (SIEPEN, 1998). Nach OLFS (1992) lässt die $N_{\text {org }}$-Fraktion nur eine eingeschränkte quantitative Abschätzung der N-Nachlieferung zu. Das bedeutet, dass die $\mathrm{N}_{\text {org-Methode sich nicht }}$ ohne weiteres für die Bedarfsprognose einsetzen lässt.

Ausgehend von dieser Situation schlagen WERNER und WODSAK (1992) einen verbesserten Ansatz vor. Dieser Ansatz besteht aus der Verbindung der $\mathrm{N}_{\text {org }}{ }^{-}$ Methode mit der von SCHARPF und WEHRMANN (1979) entwickelten $\mathrm{N}_{\text {min }}{ }^{-}$ Methode. In diesem Düngungskonzept geht es darum, Standortunterschiede bezüglich des Stickstoffnachlieferungspotentials zu ermitteln, die mit der frühen $\mathrm{N}_{\text {min- }}$ Methode (Probe im März) gar nicht und mit der späten $\mathrm{N}_{\min }$-Methode (Probe im Mai/Juni) nur teilweise erfasst werden (SIEPEN, 1998). WERNER (1991) wies durch zahlreiche Versuche im Rheinland und im westlichen Münsterland (Kreis Steinfurt) nach, dass die $\mathrm{N}_{\text {org}}$-Gehalte der Böden in einem Naturraum einer Normalverteilung entsprechen und die Aufstellung eines Gehaltsklassensystems möglich ist. Auf Basis dieser Gehaltsklassen untersuchte SIEPEN (1998), ob es sinnvoll ist, je nach $\mathrm{N}_{\text {org- }}{ }^{-}$ Gehaltsklasse Zu- oder Abschläge zur Düngung zu machen.

Die Ergebnisse der Untersuchung von SIEPEN (1998) im Frischhofsbachgebiet zeigten, dass der Zuschlag zur Düngung bei einer niedrigen $\mathrm{N}_{\text {org}}$-Gehaltsklasse problematisch ist. Hierbei stiegen nämlich die Rest $\mathrm{N}_{\text {min }}$-Gehalte im Herbst leicht an und die zusätzliche Stickstoff Düngermenge setzte sich nicht dementsprechend in Ertrag um. Bei Flächen, die in der mittleren $\mathrm{N}_{\text {org }}$-Gehaltsklasse liegen, reicht laut SIEPEN (1998) auch auf Dauer die $\mathrm{N}_{\min }$-Methode aus.

\section{Nitracheck}

Neben den Methoden, die den Boden untersuchen, gibt es solche, die die Pflanze analysieren. Hierzu gehört das Nitracheck-Verfahren. Dabei wird der Nitratgehalt im Pflanzensaft gemessen (NITSCH, 1992). Der Nitratgehalt eignet sich nach Meinung von JAKOB et al. (1986) besonders gut für die Bestimmung des Stickstoffversorgungsgrades bei Mais. Die zu untersuchenden Proben werden der Maispflanze an der Stengelbasis (NITSCH, 1991) oder an den Blattscheiden der basalen Blätter entnommen (GEYER und MARSCHNER, 1990). Sinkt der Nitratgehalt Z.B. im Entwicklungsstadium 22 des Maises unter das von NITSCH (1997) angegebene Optimum (<3000ppm), so war die Düngung vor der Saat nicht ausreichend und es kann im gewissen Rahmen nachgedüngt werden. Nach 
Aussagen von NITSCH $(1992,1999)$ ist es mit diesem Verfahren möglich, die NDüngermengen drastisch zu reduzieren.

\section{- Düngemittelapplikation und Wirkung}

Neben der Ermittlung der optimalen Düngermenge stehen auch Maßnahmen zur Verbesserung der räumlichen und zeitlichen Verfügbarkeit des Düngestickstoffs im Mittelpunkt vieler Forschungsarbeiten. Aufgrund der unterschiedlichen Beschaffenheit und den daraus resultierenden Besonderheiten der Applikationstechnik wird im Folgenden zwischen mineralischen und organischen $\mathrm{N}$ Düngern differenziert.

\section{Mineralische Düngung}

Hinsichtlich der mineralischen Düngung empfehlen ZSCHEISCHLER et al. (1990) auf bindigen Böden die Düngung in einer Gabe vor der Saat zu verabreichen und auf leichten Standorten die Düngung zu teilen. MAIDL (1997) berichtet darüber, dass eine Zweiteilung der Stickstoffdüngung (50\% vor der Saat und $50 \%$ im 6Blattstadium) zu höheren Aufnahmeraten des Maises und zur Reduzierung der Auswaschungsgefahr beitragen kann. Ein Einfluss der Teilung von

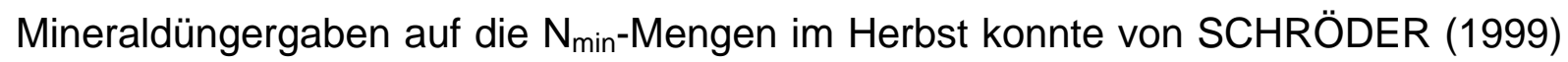
nicht festgestellt werden. Durch den Einsatz von stabilisierten N-Düngern (Alzon) lassen sich nach SCHEFFER (1994) die Nitratausträge in die Vorfluter verringern. SCHEFFER und BARTELS (1998) konnten keine permanente Reduzierung der Herbst $\mathrm{N}_{\min }$-Mengen durch den Einsatz von stabilisierten N-Düngern feststellen. Die mineralische Stickstoffdüngung wird gegenüber der organischen bezüglich der Nitratauswaschung als weniger gefährlich betrachtet, da diese gezielt zu den Aufnahmephasen der jeweiligen Kultur verabreicht werden kann (RIEß, 1993). Wie bei der Gülledüngung kann es auch bei der mineralischen Düngung zu Ammoniakverlusten kommen. Welche Düngemittel stärker Verlust gefährdet sind und nach der Ausbringung am besten eingearbeitet werden sollten, untersuchte AMBERGER (1989). Da sich der Maisanbau in den Veredelungsgebieten konzentriert, beschränkt sich dort die mineralische Düngung häufig auf die Unterfußdüngung (JACOBS, 1998). Nach Aussage von LÜTKE ENTRUP et al. (1995b) wird in nur 2,4\% der maisanbauenden Betriebe in NRW rein mineralisch gedüngt. 


\section{Organische Düngung}

Die organische Düngung zum Mais unterliegt erheblichen Stickstoffverlustrisiken (MAIDL et al., 1999). Insbesondere die falsche Terminierung der Ausbringung von Gülle gilt hinsichtlich der Nitratauswaschung als problematisch (VETTER und STEFFENS, 1986). Durch die Ausbringung der Gülle kurz vor der Saat ist nach RIEß (1993) die Nährstoffausnutzung am höchsten, entsprechend sind die N-Verluste am geringsten. Eine Teilung der Güllegaben (50\% vor der Saat / 50\% im 6 Blatt Stadium des Maises) wirkt sich nach MAIDL (1997) positiv auf die N-Aufnahme und Verwertung aus. Ein Einfluss der Teilung von Güllegaben (50\% vor der Saat / 50\% im 4-6 Blatt Stadium des Maises) auf die $\mathrm{N}_{\min }$-Mengen im Herbst konnte von SCHRÖDER (1999) nicht festgestellt werden. Nach HOEGEN und WERNER (1998) werden insbesondere auf den mit Gülle gedüngten Praxisflächen höhere Rest $\mathrm{N}_{\text {min- }}$ Gehalte gefunden als auf den mineralisch gedüngten. JOKELA (1992) fand dagegen bei einer Düngung mit Rindermist oder mineralischem Dünger ähnliche Herbst $\mathrm{N}_{\text {min- }}$ Gehalte. LORENZ et al. (1991) stellten fest, dass bei einer optimal applizierten Gülledüngung die $\mathrm{N}_{\min }$-Mengen nach der Maisernte bemerkenswert gering sind. So treten bei der Gülledüngung hauptsächlich Auswaschungen auf, wenn die Gülle zu einem ungünstigen Zeitpunkt ausgebracht oder aber das Düngeoptimum überschritten wird (VETTER und STEFFENS, 1986).

Die warme Witterung im April zur Maisaussaat begünstigt $\mathrm{NH}_{3}$-Verluste bei der Ausbringung von Flüssigmist (HORLACHER und MARSCHNER, 1990). MANNHEIM et al. (1995) und VALTA (1996) zeigten, wie wichtig eine sofortige Einarbeitung der ausgebrachten Gülle zur Reduzierung der gasförmigen N-Verluste ist. Zu ähnlichen Aussagen kamen KIRCHMANN und LUNDVALL (1998) bei der Ausbringung von Festmist. Wird Gülle hingegen nicht sofort eingearbeitet, so weisen $\mathrm{DOSCH}$ und GUTSER (1995a, 1995b) darauf hin, dass die $\mathrm{NH}_{3}$ Emissionen in Bezug auf die Ausbringungstechnik vom Prallteller über den Schleppschlauchverteiler hin zur Injektion abnehmen.

Der Stickstoff liegt in der Gülle in Abhängigkeit von Tierart, Lagerung und Aufbereitungsverfahren zu etwa 40-80\% in Form von Ammoniumstickstoff, der übrige Anteil in organischen Verbindungen vor (VETTER und STEFFENS, 1986). Der Ammoniumstickstoff ist unter vergleichbaren Anwendungsbedingungen genauso gut pflanzenverfügbar wie der in Mineraldüngern. Der organisch gebundene Stickstoff wird nur zum Teil im Anwendungsjahr pflanzenverfügbar (VETTER und STEFFENS, 
1986). Daher kann nach MAIDL et al. (1999) Gülle, die sofort eingearbeitet wird, eine sehr hohe Mineraldüngeräquivalenz (MDÄ) aufweisen. Ebenso zeigte sich in den Versuchen, dass eine Gülleausbringung vor der Saat mit unverzüglicher Einarbeitung am effektivsten ist. Laut VALTA (1999) und EBERDORFER (2002) ist es möglich, mineralischen Stickstoff komplett durch Gülle zu ersetzen. Durch eine sofortige Einarbeitung der Gülle ist auch beim Einsatz von unterschiedlicher Applikationstechnik kein Unterschied im Ertrag feststellbar (EBERDORFER, 2002).

\subsubsection{Acker- und pflanzenbauliche Maßnahmen zur Minderung von N- Austrägen}

\section{- Integration von Boden- und Wasserschutzmaßnahmen in die Fruchtfolge}

Mais ist eine der wenigen Früchte, die den Boden lediglich 5 Monate effektiv bedeckt halten. Gerade in der restlichen Zeit sind die Ackerflächen - je nach Fruchtfolgegestaltung - unbedeckt und der Stickstoff, der mineralisiert, ist auswaschungsgefährdet (PUHL, 1987; LÜTKE ENTRUP und ZERHUSEN, 1992).

Eine möglichst lange und wirksame Bodenbedeckung mit ausreichender Nitratkonservierung kann durch Zwischenfrüchte erzielt werden (ANTONY et al., 2001; LÜTKE ENTRUP, 2001). Zur Reduzierung der Nitratauswaschung vor dem Maisanbau erwiesen sich die winterharten Zwischenfrüchte gegenüber den abfrierenden Zwischenfrüchten als die bessere Wahl (PERETZKI, 1998). So können abfrierende Zwischenfrüchte im Zeitraum Vegetationsende der Zwischenfrucht bis Vegetationsbeginn des Maises bis zu 80\% der vor dem Winter gespeicherten NMenge wieder freisetzen (BERGER et al., 1993). Durch den Einsatz von winterharten Zwischenfrüchten (Grünroggen / Weidelgras) vor dem Maisanbau kam es bei MARTINEZ und GUIRAUD (1990) und TOLLENAAR et al. (1993) zu Ertragsminderungen beim Silomais. Sollte der Aussaattermin des Maises auf Grund der Aberntung der Zwischenfrucht nicht mehr optimal gestaltet werden können, ist laut OLNESS et al. (1990) auch mit Ertragseinbußen zu rechnen. RENIUS et al. (1992) und WEISE et al. (1994) stellten dagegen einen positiven Ertragseinfluss von Zwischenfrüchten auf die anschließende Hauptfrucht fest.

Nach JACOBS et al. (1996) lässt ein üppig entwickeltes Ausfallgetreide hinsichtlich der Stickstoffbindung ähnliche Effekte erwarten wie eine misslungene Zwischenfrucht vor dem Maisanbau. 
Zur Reduktion der Nitratwerte nach der Maisernte können Begrünungsmaßnahmen durchgeführt werden. Hierfür eignen sich Untersaaten oder Grünroggen (HUGGER, 1989; STEMANN, 1992; SCHRÖDER et al., 1996). Ein Vergleich der Grasuntersaat mit einer Grünroggenansaat nach Mais von VAN DIJK et al. (1997) zeigt, dass die Grasuntersaat die Nitratauswaschung stärker reduziert. Dieses ist nach VAN DIJK et al. (1997) dadurch zu erklären, dass zur Grasuntersaat keine Bodenbearbeitung mehr durchgeführt wird und die Graswurzeln mehr Stickstoff binden. Im Durchschnitt der Jahre errechnet sich unter niederländischen Bedingungen eine N-Aufnahme der Untersaat und des Grünroggens von ungefähr $40 \mathrm{~kg} / \mathrm{ha}$ (VAN DIJK et al., 1997). BÜCHTER (2003) stellt unter norddeutschen Bedingungen nur eine geringe Wirkung der Untersaaten im Hinblick auf die Reduzierung des N-Austrages fest. Für BECKER (1999) wiederum zählt der Einsatz von Zwischenfrüchten und Untersaaten zu einer der wichtigsten Maßnahmen zur Senkung der Herbst $\mathrm{N}_{\min }$-Gehalte.

In den Untersuchungen von HUGGER (1989), STEMANN und LÜTKE ENTRUP (1990) wurden keine Ertragsverluste durch die Untersaat beim Mais festgestellt. JOVANOVIC et al. (2000) stellten hingegen starke Ertragseinbußen fest. Die untersaatbedingten Ertragseinbußen traten bei JOVANOVIC et al. (1999) umso stärker auf, je geringer die N-Versorgung der Maisbestände war. Auf Standorten mit geringer Bodenkrume und eingeschränktem durchwurzelbaren Bereich kann es zur Wasserkonkurrenz zwischen der Untersaat und dem Mais kommen (STEMANN, 1992).

\section{- Variation der Pflanzenabstände}

Der Maisanbau erfolgt heute überwiegend mit einem Reihenabstand von $75 \mathrm{~cm}$, was unter anderem an der in der Praxis verfügbaren Sä- und Erntetechnik liegt (PEYKER, 2000). Untersuchungen von LORENZ (1992) zeigten, dass nach der Maisernte die $\mathrm{N}_{\text {min }}$-Gehalte zwischen den Reihen häufig höher waren als in den Reihen. Nach Ansicht von MAIDL (1990) liegt das an der geringen Wurzeldichte zwischen den Reihen. So fanden AUFHAMMER und KÜBLER (1997) heraus, dass die Bodendurchwurzelung und die N-Aufnahme von der Pflanzenzahl erheblich beeinflusst werden kann. In Versuchen mit Anbau von Mais in Doppelreihen zeigten sich über die gesamte Probennahmetiefe gleiche $\mathrm{N}_{\min }$-Gehalte in der Maisreihe und im Zwischenreihenbereich. Dieses deutet auf eine intensivere Durchwurzelung, verbunden mit einer umfangreichen Nitrataufnahme im Zwischenreihenbereich hin. In 
den Versuchen von PEYKER (1998 / 2000 / 2004) mit verringerten Reihenweiten zeigte sich eine eindeutige Reduzierung der $\mathrm{N}_{\min }$-Gehalte im Boden bei einer Reihenweite von $30 \mathrm{~cm}$ und 10 Pflanzen je $\mathrm{m}^{2}$. In Versuchen der LANDWIRTSCHAFTSKAMMER WESER-EMS (1999) trat bei einer Düngungsstufe von $130 \mathrm{~kg} \mathrm{~N}$ je ha ein positiver, die $\mathrm{N}_{\min }$-Gehalte reduzierender Effekt der $30 \mathrm{~cm}$ Reihenweite auf. Dieser Effekt verringerte sich bei einem hohen N-Angebot (möglicherweise auch bei starker N-Nachlieferung) sowie bei einer verminderten $\mathrm{N}$ Zufuhr. RIECKMANN et al. (2003) konnten anhand des herbstlichen $\mathrm{N}_{\min }$-Gehaltes kein klares Ergebnis aufzeigen. So wurden in den hoch gedüngten Varianten bei engeren Reihenweiten sogar höhere Restnitratwerte als bei der $75 \mathrm{~cm}$ Reihenweite gefunden. Gar keinen Einfluss zeigte die Reihenweitenvariation bei MÜLLER et al. (2003) auf einem stark mineralisierenden Standort. Bei Versuchen zur Gleichstandsaat von HAHNENKAMM et al. (2000) konnten bei einem Düngungsniveau von $160 \mathrm{~kg} \mathrm{~N} /$ ha keine Unterschiede bei dem herbstlichen $\mathrm{N}_{\text {min- }}$ Gehalt im Vergleich zu der $75 \mathrm{~cm}$ Reihenweite festgestellt werden.

\section{- Bodenbearbeitung}

Die Bodenbearbeitung hat nach LOCHMANN et al. (1989) einen großen Einfluss auf die Mineralisierungshöhe und den Mineralisierungsverlauf. Da die Mineralisationsrate von der Dichtlagerung des Bodens abhängt, ist bei einer Minimalbodenbearbeitung von einer geringeren Mineralisationsrate auszugehen (BAEUMER, 1992; ESTLER und KNITTEL, 1996). GUTSER und VILSMEIER (1988) weisen auf die Möglichkeiten der Minderung der Nitratauswaschung hin. Die Versuche von NEUBAUER und KOHLS (1994) zeigten, dass tiefes Pflügen im Herbst für den Anbau von Mais zu einer starken Stickstoffverlagerung führt. Dagegen bewirkt tiefes Pflügen im Frühjahr einen starken Mineralisationsschub zu Vegetationsbeginn gegenüber der ausgeglichenen N-Nachlieferung bei einer konservierenden, stark aufwandsgeminderten Bodenbearbeitung. Ein ähnliches Ergebnis stellte auch ROTTMANN (1996) fest. Bei der Mulchsaat von Mais ohne Bodenbearbeitung fand eine gleichmäßige und geringere Mineralisation statt. Laut THOMAS et al. (1973) ist bei Mulchsaat eine höhere N-Düngung als bei konventioneller Aussaat erforderlich. Beim Mulchsaatverfahren ist häufiger mit einem Ertragsrückgang im Vergleich zur Pflugbearbeitung zu rechnen (GUTSER und MANHART, 1990; BECKER et al., 1995; ROTTMANN, 1996). Dass eine konservierende Bodenbearbeitung generell auch zu 
einer Verminderung des $\mathrm{N}_{\min }$-Gehaltes im Herbst führt, konnte LANG (1992) in seinen Versuchen nicht nachweisen.

\section{- Sortenwahl}

Zur Minderung der Nitratbelastung kann auch der Einsatz N-effizienter Sorten beitragen. Nach PRESTERL und THIEMT (1999) ist die N-Effizienz eine genetisch verankerte Eigenschaft. In Versuchen von PRESTERL et al. (1994) zeigte sich, dass die N-effizienten Hybriden bei N-Mangel in der Lage sind, mehr Stickstoff aus dem Boden aufzunehmen. Durch diese Eigenschaft würden sich bei angepasster Düngung niedrigere Restnitratwerte und damit geringere Auswaschungsverluste erreichen lassen. Auch WIESLER und HORST (1988) fanden heraus, dass es sortenbedingte Unterschiede bei der Nutzung des Stickstoffangebotes durch den Mais und die darauf folgenden Nitrat-Restmengen gibt. MÜLLER et al. (2003) stellten Einflüsse des Sortentypes auf die N-Austräge fest, die sich nicht allein durch die NEntzüge erklären ließen. Eine Abschätzung des Minderungspotentials in der landwirtschaftlichen Praxis durch Sortenwahl ist derzeit jedoch nicht möglich, da die $\mathrm{N}$-Effizienz der verbreitet angebauten Sorten bisher nicht untersucht wurde (PRESTERL und THIEMT, 1999).

\subsection{Methoden zur Erfassung der Nitrataustragsgefährdung beim Maisanbau}

Zur Erfassung der Nitrataustragsgefährdung aus Ackerflächen über Winter gibt es verschiedene Ansätze.

Durch die $\mathrm{N}_{\min }$-Beprobung im Herbst lässt sich der Mineralstickstoffgehalt in der Wurzelzone vor der winterlichen Sickerwasserneubildung bestimmen. Aus dem Herbst $\mathrm{N}_{\text {min }}$-Gehalt und der Sickerwasserrate ist es möglich, die zu erwartende $\mathrm{NO}_{3}$ Anlieferung an das Grundwasser abzuschätzen (ANTONY et al., 2001). Dagegen ermöglicht die Verwendung von Saugkerzen und Lysimetern eine direkte stofflichanalytische Kontrolle der Sickerwasserqualität unter den Maisanbauflächen (ANTONY et al., 2001). Beim Einsatz von Saugkerzen und Lysimetern ist es möglich, Zeitreihen der Sickerwasserqualität zu erheben (ANTONY et al., 2001). 


\section{$\mathrm{N}_{\min }$-Methode}

Seit vielen Jahren wird die $\mathrm{N}_{\text {min }}$-Menge des Bodens im Herbst als Kontrollinstrument für die Austragsgefährdung durch Landbewirtschaftung eingesetzt (z.B. SchALVO). Der Herbst $\mathrm{N}_{\min }$-Gehalt beschreibt den Mineralstickstoffgehalt in der Wurzelzone vor Beginn der winterlichen Sickerwasserneubildung. $\mathrm{Er}$ ist abhängig vom Pflanzenentzug, der N-Mineralisation, der N-Immobilisation, der Denitrifikation, der Nitratauswaschung und eventueller Düngung (BÜCHTER, 2003; ANTONY, 2001). Die Erfassung des Herbst $\mathrm{N}_{\text {min}}$-Gehaltes ist das einzige großflächig einsetzbare stofflich-analytische Verfahren, um mit vertretbarem Aufwand Aussagen über die auswaschungsgefährdeten Stickstoffmengen in der Wurzelzone zu erhalten (ANTONY, 2001). Mit Hilfe von $\mathrm{N}_{\min }$-Daten ist nach SIMON et al. (1988) und BÜCHTER (2003) eine Abschätzung von Nitratausträgen möglich, die mit den Ergebnissen von Saugkerzen vergleichbar sind. Eine Korrelation $(r=0,74)$ zwischen dem herbstlichen $\mathrm{N}_{\text {min }}$-Gehalt und der Auswaschung (Dränwasser) konstatierten auch GÖLZ-HUWE et al. (1989). Nach KNOBLAUCH et al. (1999) ist die Beziehung zwischen der Herbst $\mathrm{N}_{\text {min-Menge }}$ und dem Nitratgehalt im Sickerwasser stark vom Standort abhängig. ALBERT und LIPPOLD (1997) stellten eine enge Beziehung zwischen dem $\mathrm{N}_{\min }$-Niveau und der Einwaschung ins Unterflurlysimeter fest.

\section{Saugkerzen-Methode}

Saugkerzen werden seit ca. 45 Jahren zur Gewinnung von versickerndem Bodenwasser eingesetzt (DVWK, 1990). Durch die Installation einer Saugkerze wird der Boden um die Kerze herum gestört (BECKMANN et al., 1991). Mittels eines erzeugten Unterdruckes wird die Bodenlösung durch die Keramik in das Innere der Saugkerze geleitet und kann so zur Analyse des Nitratgehaltes herangezogen werden (RIEß, 1993). Nach WEIHERMÜLLER et al. (2003) wird der Einflussbereich einer Saugkerze wesentlich von der Textur und der Heterogenität des Bodens sowie der Infiltration und der Transevaporation mitbestimmt. Die Saugkerzen können Bodenwasser auffangen, das sich in der Transportform des laminaren Flusses entlang der Bodenfestphase befindet, jedoch nicht durch Makroporen schnell versickerndes Wasser (SCHMIDT, 1992; KÜCKE et al., 2001). Insbesondere in strukturierten tonhaltigen Böden kann die Versickerung teilweise über Makroporen erfolgen (ADDISCOTT, 1996; GOULDING und WEBSTER, 1992). Zur Berechnung der Nitratfrachten wird die Sickerwassermenge benötigt, welche aus aktuellen 
Wetterdaten berechnet werden kann (BENKE, 1992; RIEß, 1993). Die mit der Saugkerzen-Methode ermittelten Nitratfrachten sind nach WEBSTER et al. (1993) mit denen von Lysimeteranlagen vergleichbar.

\section{Lysimeter}

Durch Lysimeter kann das perkolierende Sickerwasser an einer definierten Grenze aufgefangen werden (ANTONY et al., 2001). Lysimeter sind in der Regel unten und seitlich umwandete Bodensäulen mit einer am Boden befindlich Vorrichtung zur Entnahme von Sickerwasser (ROTH und WERNER, 2000). Durch die Entnahme von Sickerwasser ist somit auch die Nitratauswaschung einer Bodensäule direkt messbar. Die Sickerwassermenge ist von dem Speichervermögen des Bodens für Wasser und nicht zuletzt vom Pflanzenbestand abhängig (AMBERGER, 1996). Lysimeteranlagen gibt es in verschiedenen Größen. Sie können eine Grundfläche von 1-4 $\mathrm{m}^{2}$ und eine Tiefe von 2-3 $\mathrm{m}$ aufweisen (SCHILLING, 2000). Die Zylinderform wird heute bei den Lysimeterkörpern bevorzugt (LEIS et al., 2001). Es gibt wägbare und nicht wägbare Lysimeter und solche mit und ohne Grundwasser (ANTONY et al., 2001). Zu welchen seitlichen Randeffekten es beim Betreiben von Lysimetern kommen kann, untersuchten KRENN et al. (2003). Der Einsatz von Monolithlysimetern sollte den gestört befüllten Lysimetern vorgezogen werden, da eine Befüllung nicht die natürlichen Bodeneigenschaften repräsentiert (LEIS et al., 2001). Nach SEIDLER und KLOTZ (1999) bringen Lysimeteruntersuchungen nur bedingt quantitativ übertragbare Ergebnisse für die Region, da sie nicht in großräumige Messnetze eingebunden sind und häufig für den Beobachtungsort untypische Füllungen/Schüttungen enthalten. Zum Prozessverständnis leisten Lysimeteranlagen dennoch einen wesentlichen Beitrag. 


\section{Material und Methoden}

\subsection{Material}

\subsubsection{Daten aus der Wasserschutzgebietsberatung (Datenkategorie 1)}

\section{Datenherkunft}

In diesem Abschnitt der vorliegenden Arbeit wurden Daten aus der Wasserschutzgebietsberatung von den Landwirtschaftskammern Weser-Ems (LWKWE), Schleswig-Holstein (LWK-SH) und dem Ingenieurbüro Geries in eine Datenbank integriert und ausgewertet.

Von der Landwirtschaftskammer Weser-Ems wurden Daten von ca. 600 im Rahmen der Wasserschutzberatung beprobten Maisflächen aus der Region Weser-Ems für die Untersuchungen zur Verfügung gestellt. Der Zeitraum der Beprobung erstreckte sich von 1994-1999. Von den ca. 600 beprobten Flächen sind für die Mehrzahl der Auswertungen 466 Flächen herangezogen worden. Die Reduzierung um 140 Flächen ist dadurch zu erklären, dass auf vielen Flächen häufiger Mais angebaut wurde und die zugehörigen Standortumwelten damit mehrfach in der Datenbank vorkommen. Da diese Mehrfachaufführung eines gleichen Standortes zu einer Fallverzerrung führen kann, die bei vielen Auswertungen verfälschend wirken könnte, wurde jeweils das verwendete Jahr einer Fläche zufällig ausgewählt (KÖHLER et al., 1995). Auf diese Art und Weise kann die geforderte Unabhängigkeit der erfassten Primärdaten sichergestellt werden, die für viele statistische Tests eine Grundvoraussetzung darstellt.

Von der Landwirtschaftskammer Schleswig-Holstein wurden Daten aus dem Pilotprojekt „Flächendeckender Gewässerschutz“ zur Verfügung gestellt. Das Pilotprojekt wurde in der Region Oeversee (Schleswig-Holstein) durchgeführt. Da auch in diesem Fall Flächen über mehrere Jahre mehrfach beprobt worden sind, wurde das verwendete Jahr einer Fläche zufällig ausgewählt. Hiernach wurden 21 Flächen, die über die Jahre 1997 bis 2000 auf fünf Betrieben näher beobachtet worden sind, in die Datenbank aufgenommen.

Das Ingenieurbüro Geries stellte Daten aus der Wasserschutzgebietsberatung zur Verfügung. Die Daten wurden im Weser-Elbe-Dreieck über die Jahre 1995 - 2001 erhoben. 13 Flächen waren über diesen Zeitraum jährlich beprobt worden. Wie schon 
bei den zuvor genannten Datenherkünften wurde für jede Fläche nur ein Beprobungsjahr zufällig ausgewählt.

Anhand der folgenden Karte soll die räumliche Verteilung der Daten aus dieser Datenkategorie dargestellt werden.

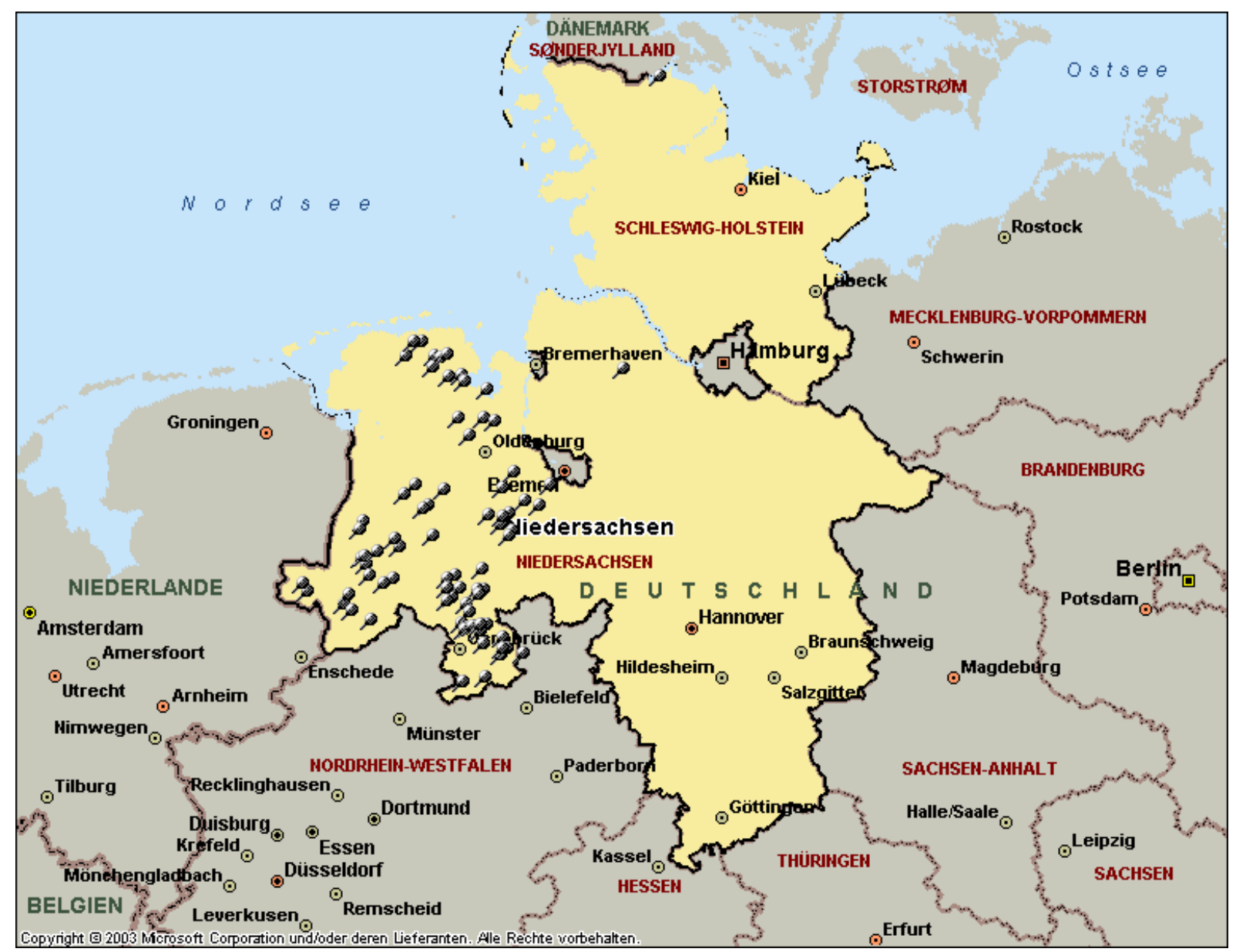

Abb. 1: Übersicht zur räumlichen Verteilung der Daten aus den Wasserschutzgebieten. 


\section{Variablen der verwendeten Daten:}

Die Daten der Landwirtschaftskammer Weser-Ems enthalten flächenbezogene Angaben über den Herbst und Frühjahrs $\mathrm{N}_{\min }$-Gehalt im Boden, den Ertrag (Schätzgröße der Berater), die Art und Höhe der Düngung (Schätzgröße aus der Düngeplanung für die Landwirte), die Vorfrucht, die Zwischenfrucht sowie die Körnung und das C/N-Verhältnis der Ackerkrume. Teilweise lagen Angaben über den GV-Besatz des die Erhebungsflächen bewirtschaftenden Betriebes vor. Zur Erhöhung der Aussagekraft der Daten wurden Nacherhebungen durchgeführt. So konnten 289 Flächen Bodenkennwerte der Reichsbodenschätzung zugeordnet werden. Des Weiteren sind 100 Flächen nachträglich auf deren Gehalte an GesamtStickstoff und Kohlenstoff in der Krume $(0-30 \mathrm{~cm})$ beprobt worden.

Den Daten der Landwirtschaftskammer Schleswig-Holstein fehlten die Angaben zu den Erträgen, dem Zwischenfruchtanbau und dem Viehbesatz. Ansonsten sind die gleichen Kenngrößen wie bei der Landwirtschaftskammer Weser-Ems erhoben worden. Die Düngermengen wurden in diesem Fall exakt aufgenommen.

Bei den Daten des Ingenieurbüros Geries sind die Kenngrößen der Landwirtschaftskammer Weser-Ems bis auf den Ertrag vorhanden. Die C/NVerhältnisse wurden auch hier nachträglich beprobt. Für alle Flächen wurden über den Deutschen Wetterdienst die täglichen Messwerte der Temperatur, des Niederschlages und der rel. Luftfeuchte der nächstgelegenen Klimastation beschafft. Die Variablen und die Anzahl der Werte je Variable für die verschiedenen Datenherkünfte werden in Tab. 1 gezeigt.

Tab. 1: Zusammensetzung der Daten aus den Wasserschutzgebieten nach der Randomisation (Datenkategorie 1)

\begin{tabular}{|c|c|c|c|}
\hline Variable & Weser-Ems & $\begin{array}{c}\text { Anzahl } \\
\text { Schleswig-Holstein }\end{array}$ & Weser-Elbe-Dreieck \\
\hline Frühjahr $\mathbf{N}_{\min }$ & 226 & 19 & 5 \\
\hline Herbst $\mathbf{N}_{\min }$ & 392 & 13 & 13 \\
\hline Ertrag (Schätzgröße) & 461 & 0 & 0 \\
\hline Düngung (Schätzgröße) & 466 & 21 & 13 \\
\hline C/N-Verhältnis & 141 & 19 & 13 \\
\hline $\begin{array}{l}\text { Bodenart (aus der } \\
\text { Reichsbodenschätzung) }\end{array}$ & 289 & 20 & 13 \\
\hline $\begin{array}{l}\text { Bodenart (Angabe des } \\
\text { Beraters) }\end{array}$ & 211 & 20 & 13 \\
\hline $\begin{array}{l}\text { GV-Besatz } \\
\text { (< oder }>2 \text { GV/ha) }\end{array}$ & 178 & 0 & 13 \\
\hline Vorfrucht & 419 & 16 & 13 \\
\hline Zwischenfrucht & 434 & 0 & 3 \\
\hline $\begin{array}{l}\text { Tägliche Klimadaten } \\
\text { (Temperatur I Nieder- } \\
\text { schlag I Luftfeuchte I } \\
\text { Evapoptranspiration) }\end{array}$ & 466 & 21 & 13 \\
\hline
\end{tabular}




\subsubsection{Daten aus Erhebungen (Datenkategorie 2)}

\section{Datenherkunft}

In die Datenkategorie 2 wurden drei überregionale bzw. bundesweite Erhebungen aufgenommen. Es wurden Daten aus dem $\mathrm{N}_{\text {mon-Projekt des }}$ Bundesarbeitskreises Düngung (BAD) und des Verbandes Deutscher Landwirtschaftlicher Untersuchungsund Forschungsanstalten (VDLUFA), dem Vergleichsflächenprogramm der Landwirtschaftlichen Untersuchungs- und Forschungsanstalt Augustenberg (LUFA) und dem Nitratrasterprogramm der Landwirtschaftskammern Weser-Ems (LWK-WE) und Hannover (LWK-HAN) für diese Untersuchung zur Verfügung gestellt. Im Gegensatz zum $\mathrm{N}_{\text {mon }}$-Projekt wurden die Erhebungen der LUFA Augustenberg und der Landwirtschaftskammern Hannover und Weser-Ems nicht bundesweit, sondern länderspezifisch durchgeführt.

Aus dem $\mathrm{N}_{\text {mon }}$-Projekt sind Daten von 278 Flächen, auf denen Mais in den Jahren 1999 oder 2000 angebaut wurde, bereitgestellt worden. Die LUFA Augustenberg stellte Daten aus den Jahren 1988 bis 2000 bereit. Diese stammten aus dem Vergleichsflächenprogramm. Insgesamt wurden Daten von 532 beprobten Flächen zur Verfügung gestellt. Dieses sind ungefähr 44 Flächen je Jahr. Von den Landwirtschaftskammern Hannover und Weser-Ems sind Daten aus der Nitratrastererhebung aus den Jahren 1985 bis 1987 eingebracht worden. Diese umfassten ca. 20-30 Erhebungsflächen je Jahr, auf denen Mais angebaut wurde.

Von den insgesamt 886 über die Jahre 1985 - 2000 beprobten Flächen wurden für die Auswertungen 414 Flächen herangezogen. Die Reduzierung um 472 Flächen ist dadurch zu erklären, dass auf manchen Flächen häufiger Mais angebaut wurde und diese damit doppelt in der Datenbank vorkamen. Da diese doppelten Flächen die Auswertungen bzgl. des Standortes beeinflussen und dadurch $\mathrm{zu}$ einer systematischen Verfälschung der Ergebnisse führen, wurde jede Fläche nur mit einem Beprobungsjahr in die Auswertung genommen. Die Auswahl erfolgte zufällig (KÖHLER et al., 1995). Hierdurch wird eine Unabhängigkeit erreicht, die in vielen statistischen Tests Voraussetzung ist. Einige weitere Flächen wurden aufgrund von unterschiedlichen Einflussfaktoren nicht einbezogen. Nach Aussagen der Institutionen, die Daten zur Verfügung stellten, traten auf manchen Flächen Maiszünsler- oder Wildschäden auf. Andere Flächen waren zeitweilig überschwemmt und einige durch den Flächennachbarn zusätzlich mit Stickstoff versorgt worden. Des 
Weiteren waren Flächen aus dem Vergleichsflächenprogramm der LUFA Augustenberg der BAD / VDLUFA im Rahmen des $\mathrm{N}_{\text {mon-Projektes zur Verfügung }}$ gestellt worden. Daher wurden nach Gesprächen mit beiden Institutionen diese aus dem Datensatz entfernt.

Die in Abb. 2 gezeigte Karte stellt die geografische Lage der Flächen, die in diese Datenkategorie eingeflossen sind, dar.

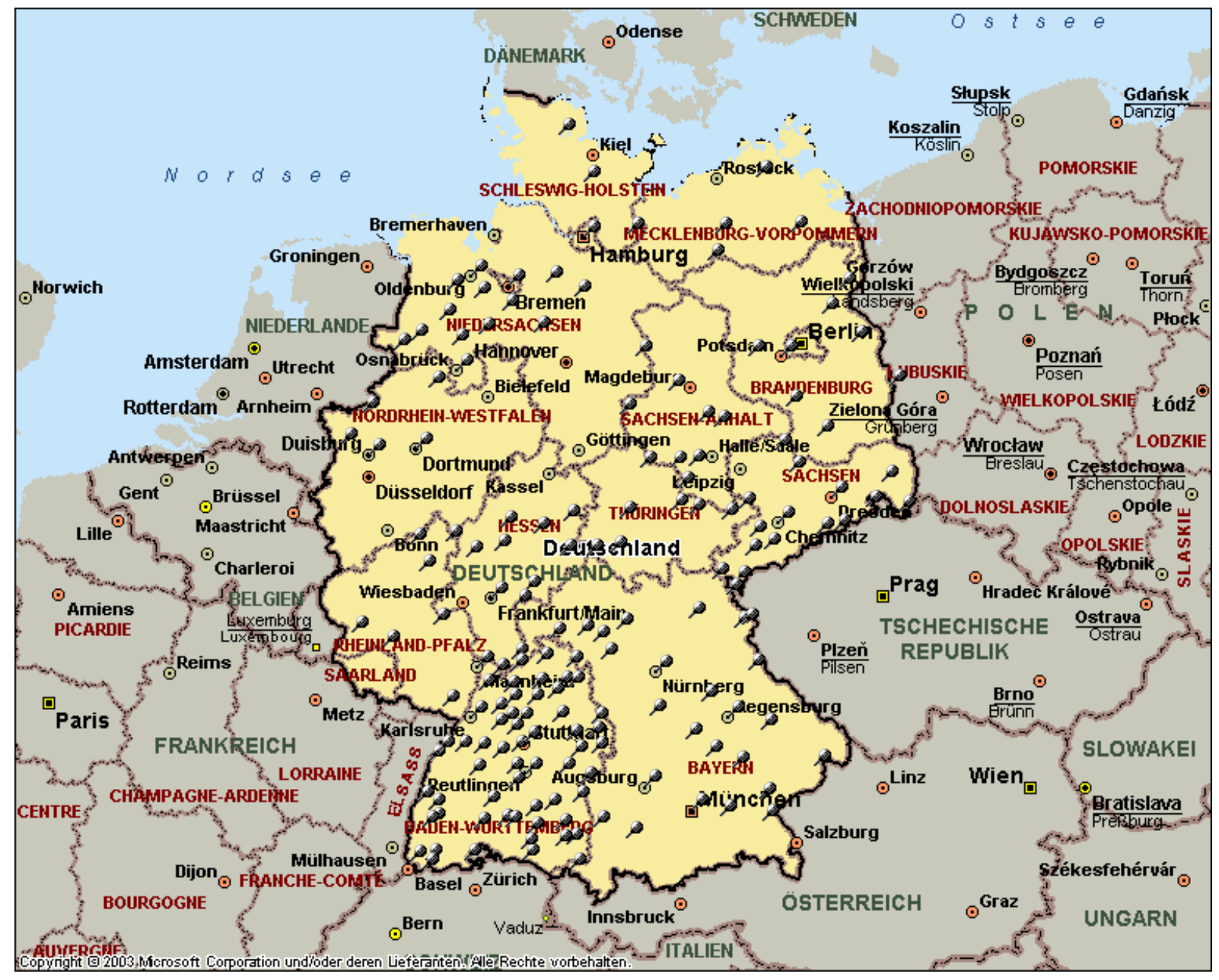

Abb. 2: Übersicht zur räumlichen Verteilung der Daten aus den Erhebungen.

\section{Variablen der verwendeten Daten}

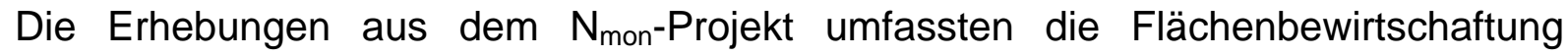
(Vorfrucht, Nebenernteprodukte, Zwischenfrucht, Erntemenge, Rohproteingehalt), Bodenuntersuchungen ( $\mathrm{pH}, \mathrm{P}_{2} \mathrm{O}_{5}, \mathrm{~K}_{2} \mathrm{O}, \mathrm{MgO}, \mathrm{C}_{\text {org }}, \mathrm{N}_{\mathrm{t}}, \mathrm{N}_{\text {min }}$ ), Angaben zur Bodenart und Ackerzahl, die mineralische und organische Düngung, sowie die Wetterdaten. Die mitgelieferten Wetterdaten aus dem $\mathrm{N}_{\text {mon }}$-Projekt wurden jedoch verworfen, da es sich um Monatswerte handelte. Allen drei Erhebungen wurden exakte tägliche 
Wetterdaten vom Deutschen Wetterdienst zugeordnet. Die Wetterdaten umfassten die Temperatur, den Niederschlag und die relative Luftfeuchte. Aus diesen Daten wurde dann die Verdunstung nach HAUDE (HÄCKEL, 1999) berechnet.

$\mathrm{Da}$ aus dem Vergleichsflächenprogramm auch Daten für das $\mathrm{N}_{\text {mon }}$-Projekt geliefert wurden, unterscheidet sich die Datenzusammensetzung nicht wesentlich. Sie differiert zumeist nur darin, dass im Vergleichsflächenprogramm keine Erhebungen des $\mathrm{C} / \mathrm{N}-$ Verhältnisses und der Grundnährstoffdüngung gemacht worden sind. Inwieweit die Daten aus dem Nitratrasterprogramm von dieser Datenzusammensetzung abweichen, geht aus der Tab. 2 hervor.

Tab. 2: Zusammensetzung der Daten von den Erhebungsflächen nach der Randomisation (Datenkategorie 2)

\begin{tabular}{|c|c|c|c|}
\hline Variable & $\begin{array}{c}\mathrm{N}_{\text {mon }} \text {-Projekt } \\
\text { BAD / VDLUFA }\end{array}$ & $\begin{array}{c}\text { Anzahl } \\
\text { Vergleichsflächen } \\
\text { LUFA Augustenberg }\end{array}$ & $\begin{array}{c}\text { Nitratraster } \\
\text { Landwirtschaftskammer }\end{array}$ \\
\hline Frühjahrs $\mathrm{N}_{\min }$ & 182 & 101 & 29 \\
\hline $\begin{array}{l}\text { Herbst } N_{\min } \\
\text { (nach Ernte) }\end{array}$ & 150 & 72 & 0 \\
\hline $\begin{array}{l}\text { Herbst } \mathrm{N}_{\min } \\
\text { (Vegetationsende) }\end{array}$ & 160 & 75 & 37 \\
\hline Ertrag & 186 & 77 & 28 \\
\hline Rohprotein & 13 & 66 & 0 \\
\hline $\begin{array}{l}\text { Düngung (Stickstoff) } \\
\text { (mineralisch + organisch) }\end{array}$ & 233 & 122 & 37 \\
\hline $\mathrm{C} / \mathrm{N}-$ Verhältnis & 159 & 0 & 37 \\
\hline Humusgehalt & 169 & 95 & 37 \\
\hline $\begin{array}{l}\text { Grundbodenuntersuchung } \\
\text { (P2O5, } \mathrm{K} 2 \mathrm{O}, \mathrm{MgO})\end{array}$ & 202 & 112 & 0 \\
\hline $\begin{array}{l}\text { Grunddüngung } \\
\text { (P2O5, } \mathrm{K} 2 \mathrm{O}, \mathrm{MgO}, \mathrm{CaO})\end{array}$ & 182 & 0 & 0 \\
\hline $\mathrm{pH}$-Wert & 201 & 113 & 0 \\
\hline Ackerzahl & 251 & 81 & 0 \\
\hline $\begin{array}{l}\text { Bodenart } \\
\text { (klassifiziert) }\end{array}$ & 251 & 120 & 37 \\
\hline GV-Besatz & 0 & 62 & 0 \\
\hline Vorfrucht & 243 & 124 & 29 \\
\hline Zwischenfrucht & 241 & 109 & 0 \\
\hline Tägliche Klimadaten & 240 & 112 & 37 \\
\hline
\end{tabular}




\subsubsection{Daten aus Exaktversuchen (Datenkategorie 3)}

\section{Datenherkunft}

Nachdem zwei Datenbestände aus Erhebungen vorgestellt wurden, enthält dieser Datenbestand die Ergebnisse aus Exaktversuchen. So wurden Versuche von den Landwirtschaftskammern aus Borken, Hannover, Münster, Oldenburg, Rheinland, Schleswig-Holstein und dem Ingenieurbüro Geries aus Göttingen aufgenommen. Des Weiteren flossen Daten aus den Dissertationen von Herrn Wieker (Fachhochschule Soest) und Herrn Siepen (Universität Bonn) ein. Ein Versuch wurde der Diplomarbeit von Herrn Rottmann (Fachhochschule Soest) entnommen. Das Forschungs- und Studienzentrum für Veredelungswirtschaft Weser-Ems der Universität Göttingen stellte ebenfalls Versuche für die Auswertung zur Verfügung. Insgesamt flossen $25 \mathrm{z}$. T. mehrortige Versuche von 137 Standorten ein.

Tab. 3: Beschreibung der Daten aus Exaktversuchen

\begin{tabular}{|c|c|c|c|}
\hline Versuchsansteller & Laufzeit & Versuchsbeschreibung & Umfang \\
\hline $\begin{array}{l}\text { Landwirtschaftskammer } \\
\text { Borken }\end{array}$ & $\begin{array}{l}1995- \\
2000\end{array}$ & $\begin{array}{l}\text { Sollwertfindungsversuche auf } 27 \\
\text { Standorten auf } 6 \text { Jahre verteilt }\end{array}$ & 3 - 4 N-Stufen \\
\hline $\begin{array}{l}\text { Landwirtschaftskammer } \\
\text { Borken }\end{array}$ & 1998 & Zwischenfruchtversuch (1 Standort) & $\begin{array}{l}3 \mathrm{~N} \text {-Stufen / } \\
4 \text { verschiedene } \\
\text { Zwischenfrüchte }\end{array}$ \\
\hline $\begin{array}{l}\text { Landwirtschaftskammer } \\
\text { Rheinland }\end{array}$ & $\begin{array}{l}1992- \\
1993\end{array}$ & $\begin{array}{l}\text { Beeinflussung des Ertrages und der } \\
\text { Restnitratwerte durch veränderte } \\
\text { Reihenweiten und } \mathrm{N} \text { - Düngung. }\end{array}$ & $\begin{array}{l}2 \text { verschiedene Sorten / } 2 \\
\text { Reihenweiten / } 3 \mathrm{~N} \text {-Stufen }\end{array}$ \\
\hline $\begin{array}{l}\text { Wieker, H. } \\
\text { (FH Soest) }\end{array}$ & $\begin{array}{l}1992- \\
1994\end{array}$ & $\begin{array}{l}\text { Untersuchung zur N-Mineralisation } \\
\text { des Bodens und N-Bedarf im } \\
\text { Maisanbau unter Berücksichtigung } \\
\text { zeitabhängiger N-Sollwerte und } \\
\text { verschiedener Standorte (55 } \\
\text { Standorte / 16 -19 je Jahr) }\end{array}$ & $\begin{array}{l}3 \mathrm{~N} \text {-Stufen bei } 6 \\
\text { zeitabhängigen } \mathrm{N} \text { - } \\
\text { Sollwerten }\end{array}$ \\
\hline $\begin{array}{l}\text { Siepen, A. } \\
\text { (Uni Bonn) }\end{array}$ & $\begin{array}{l}1993- \\
1994 \\
\end{array}$ & $\begin{array}{l}\text { Stickstoffdüngungsversuch auf } 13 \\
\text { Standorten (6 im Jahr 1993) }\end{array}$ & $4 \mathrm{~N}$-Stufen \\
\hline $\begin{array}{l}\text { Landwirtschaftskammer } \\
\text { Hannover }\end{array}$ & $\begin{array}{l}1999- \\
2000\end{array}$ & $\begin{array}{l}\text { Vers. } 647 \text { : Vergleich von } \\
\text { konservierender und konventioneller } \\
\text { Bodenbearbeitung } \\
\text { (1 gleich bleibender Standort) }\end{array}$ & $\begin{array}{l}8 \mathrm{~N} \text {-Stufen je } \\
\text { Bearbeitungsvariante }\end{array}$ \\
\hline $\begin{array}{l}\text { Landwirtschaftskammer } \\
\text { Hannover }\end{array}$ & $\begin{array}{l}1999- \\
2000\end{array}$ & $\begin{array}{l}\text { Vers. 646: Vergleich von } \\
\text { konservierender (mit Untersaat) und } \\
\text { konventioneller Bodenbearbeitung } \\
\text { (1 Standort pro Jahr) }\end{array}$ & $\begin{array}{l}6 \mathrm{~N} \text {-Stufen je } \\
\text { Bearbeitungsvariante }\end{array}$ \\
\hline $\begin{array}{l}\text { Landwirtschaftskammer } \\
\text { Hannover }\end{array}$ & $\begin{array}{l}1995 \\
1996 \\
1999 \\
2000\end{array}$ & $\begin{array}{l}\text { Vers. 642: Vergleich von } \\
\text { konservierender (mit Untersaat) und } \\
\text { konventioneller Bodenbearbeitung } \\
\text { (1 gleich bleibender Standort) }\end{array}$ & $\begin{array}{l}10 \mathrm{~N} \text {-Stufen je } \\
\text { Bearbeitungsvariante }\end{array}$ \\
\hline
\end{tabular}




\begin{tabular}{|c|c|c|c|}
\hline Versuchsansteller & Laufzeit & Versuchsfrage & Umfang \\
\hline $\begin{array}{l}\text { Landwirtschaftskammer } \\
\text { Hannover }\end{array}$ & $\begin{array}{l}1995- \\
1999\end{array}$ & $\begin{array}{l}\text { Vers. 834: Bedarfsgerechte Düngung } \\
\text { zu Silomais. ( } 5 \text { gleich bleibende } \\
\text { Standorte fast jedes Jahr). Wie wirkt } \\
\text { sich Düngermenge und Zeitpunkt auf } \\
\text { die } \mathrm{NO}_{3} \text {-Restgehalte und den Ertrag } \\
\text { aus? Einfluss der Untersaat auf den } \\
\text { Restnitratgehalt im Boden, den Ertrag } \\
\text { und die Qualität bei Silomais sowie } \\
\text { auf die optimale N-Düngermenge. }\end{array}$ & $\begin{array}{l}\text { bis zu } 10 \mathrm{~N} \text {-Stufen / } 2 \\
\text { Faktoren (Untersaat } \\
\text { ja/nein) }\end{array}$ \\
\hline $\begin{array}{l}\text { Landwirtschaftskammer } \\
\text { Hannover }\end{array}$ & $\begin{array}{l}1999- \\
2001\end{array}$ & $\begin{array}{l}\text { Vers. 835: N-Düngung zu Silomais. } \\
\text { Wie wirkt sich eine org. bzw. } \\
\text { mineralische N-Düngung auf den } \\
\text { Ertrag und den Restnitratgehalt aus? } \\
\text { (2 Standorte) }\end{array}$ & $6 \mathrm{~N}-$ Stufen \\
\hline $\begin{array}{l}\text { Landwirtschaftskammer } \\
\text { Hannover }\end{array}$ & $\begin{array}{l}1989- \\
2001\end{array}$ & $\begin{array}{l}\text { Vers. 838: Dauerversuch zur N- } \\
\text { Düngung bei Silomais. Auswirkung } \\
\text { einer Silomaismonokultur mit bzw. } \\
\text { ohne Stickstoffdüngung auf den } \\
\text { Maisertrag und einige } \\
\text { Bodenfruchtbarkeitsparameter. } \\
\text { (1 Standort) }\end{array}$ & $2 \mathrm{~N}$-Stufen \\
\hline Landwirtschaftskammer & $1998-$ & Vers. 880: Beeinflussung des & $3 \mathrm{~N}$-Stufen / 3 \\
\hline Hannover & 2001 & $\begin{array}{l}\text { Ertrages und der Restnitratwerte } \\
\text { durch veränderte Reihenweiten und } \\
\mathrm{N} \text { - Düngung. (3 Standorte) }\end{array}$ & Reihenweiten \\
\hline Geries & $\begin{array}{l}1998- \\
2000\end{array}$ & $\begin{array}{l}N \text { - Düngungsversuch mit } \\
\text { praxisüblicher N-Menge und } \\
\text { reduzierter N-Menge. }(1998=8 \\
\text { Standorte } / 1999=2 \text { Standorte } / \\
2000=1 \text { Standort) }\end{array}$ & $2 \mathrm{~N}-$ Stufen \\
\hline $\begin{array}{l}\text { Landwirtschaftskammer } \\
\text { Oldenburg }\end{array}$ & $\begin{array}{l}1995- \\
1999\end{array}$ & $\begin{array}{l}\text { Der Einfluss der Fruchtart und der N- } \\
\text { Düngung auf die Erträge, die } \mathrm{N}_{\text {min }^{-}} \\
\text {Verläufe und N-Salden. } \\
\text { ( } 6 \text { gleich bleibende Standorte) }\end{array}$ & $6 \mathrm{~N}-$ Stufen \\
\hline Landwirtschaftskammer & $1997-$ & Reihenabstände und N- & $4 \mathrm{~N}$-Stufen / 3 \\
\hline Oldenburg & 1998 & $\begin{array}{l}\text { Düngungsstufen Silomais. } \\
\text { (1 Standort je Jahr) }\end{array}$ & Reihenweiten \\
\hline Fosvwe (Uni Göttingen) & $\begin{array}{l}1998- \\
1999\end{array}$ & $\begin{array}{l}\text { N-Steigerungsversuch mit } \\
\text { mineralischer und organischer } \\
\text { Düngung (1 Standort) }\end{array}$ & $4 \mathrm{~N}$-Stufen je Düngemittel \\
\hline Fosvwe (Uni Göttingen) & $\begin{array}{l}1998- \\
1999\end{array}$ & $\begin{array}{l}\text { Inwieweit wirkt sich die Bemessung } \\
\text { der N-Düngung (mineralisch und } \\
\text { organisch) nach aktuellen } \mathrm{N}_{\text {min }}{ }^{-} \\
\text {Gehalten auf die Restnitratgehalte im } \\
\text { Boden aus? (1 Standort) }\end{array}$ & $2 \mathrm{~N}$-Stufen je Düngemittel \\
\hline Fosvwe (Uni Göttingen) & $\begin{array}{l}2000- \\
2001\end{array}$ & $\begin{array}{l}\text { Beeinflussung des Ertrages und der } \\
\text { Restnitratwerte durch veränderte } \\
\text { Reihenweiten und N - Düngung } \\
\text { (1 Standort) }\end{array}$ & 2 Reihenweiten / 1 N-Stufe \\
\hline Fosvwe (Uni Göttingen) & $\begin{array}{l}2000- \\
2001\end{array}$ & $\begin{array}{l}\text { Beeinflussung des Restnitratwertes } \\
\text { durch verschiedene Sorten. } \\
\text { (1 Standort) }\end{array}$ & 5 Sorten / 1 N-Stufe \\
\hline $\begin{array}{l}\text { Landwirtschaftskammer } \\
\text { Münster }\end{array}$ & $\begin{array}{l}1992- \\
1994\end{array}$ & $\begin{array}{l}\text { Sollwertprüfungsversuch. Wie lässt } \\
\text { sich die Stickstoffdüngung zu Mais } \\
\text { unter Berücksichtigung der N- } \\
\text { Nachlieferung aus dem Boden } \\
\text { optimieren? } \\
\text { (3 gleich bleibende Standorte) }\end{array}$ & $6 \mathrm{~N}$-Stufen \\
\hline
\end{tabular}




\begin{tabular}{|c|c|c|c|}
\hline Versuchsansteller & Laufzeit & Versuchsfrage & Umfang \\
\hline $\begin{array}{l}\text { Landwirtschaftskammer } \\
\text { Münster }\end{array}$ & 1996 & $\begin{array}{l}\text { Langfristiger } \mathrm{N} \text { - } \\
\text { Extensivierungsversuch. (1 Standort) }\end{array}$ & $6 \mathrm{~N}-S t u f e n$ \\
\hline $\begin{array}{l}\text { Landwirtschaftskammer } \\
\text { Münster }\end{array}$ & $\begin{array}{l}1990- \\
1991\end{array}$ & $\begin{array}{l}\text { Stickstoffdüngung zu Körnermais. } \\
\text { (6 Standorte teilweise gleich } \\
\text { bleibend) }\end{array}$ & 8-10 N-Stufen \\
\hline $\begin{array}{l}\text { Landwirtschaftskammer } \\
\text { Schleswig-Holstein }\end{array}$ & $\begin{array}{l}1999- \\
2001\end{array}$ & $\begin{array}{l}\text { N-Düngung zu Silomais. Wie hoch } \\
\text { sind die N-Gaben zu bemessen, um } \\
\text { optimale Erträge beim Silomais zu } \\
\text { erzielen und die Nitratgehalte zu } \\
\text { minimieren? Wie wirken organische } \\
\text { Dünger im Vergleich zu } \\
\text { Mineraldünger? (1 Standort) }\end{array}$ & $8 \mathrm{~N}-$ Stufen \\
\hline $\begin{array}{l}\text { Landwirtschaftskammer } \\
\text { Schleswig-Holstein }\end{array}$ & $\begin{array}{l}1993- \\
2001\end{array}$ & $\begin{array}{l}\text { Vergleich der N-Effizienz von } \\
\text { Rindergülle und Mineraldünger im } \\
\text { Langzeitversuch. } \\
\text { (1 Standort) }\end{array}$ & $8 \mathrm{~N}-$ Stufen \\
\hline $\begin{array}{l}\text { Rottmann, L. } \\
\text { (FH Soest) }\end{array}$ & $\begin{array}{l}1993- \\
1994\end{array}$ & $\begin{array}{l}\text { Der Anbau von Mais im System der } \\
\text { konservierenden Bodenbearbeitung. } \\
\text { Vergleich von konservierender (mit } \\
\text { Zwischenfrucht) und konventioneller } \\
\text { Bodenbearbeitung (mit } \\
\text { Zwischenfrucht) und deren } \\
\text { Auswirkungen auf die } \\
\text { Restnitratgehalte. (2 Standorte) }\end{array}$ & $\begin{array}{l}4 \mathrm{~N} \text {-Stufen / } 4 \\
\text { Zwischenfrüchte / } 4 \\
\text { Ansaatvarianten }\end{array}$ \\
\hline
\end{tabular}

Da eine gemeinsame Auswertung des kompletten Datensatzes auf Grund der unterschiedlichen Versuchsansätze nicht möglich war, werden im Folgenden die Nullparzellen und Düngeparzellen separat betrachtet. In der folgenden Auswertung werden die Nullparzellen (max. Unterfußdüngung) als „ohne Düngung“ und die Düngeparzellen als „mit Düngung“ bezeichnet. Es wurden nur Parzellen ausgewählt, welche einen Reihenabstand von $75 \mathrm{~cm}$ aufwiesen, nicht konservierend bestellt wurden und keine Untersaat enthielten. Dieses wurde vor dem Hintergrund der Vergleichbarkeit mit den ersten beiden Datenkategorien beschlossen. Die verwendete Variante „mit Düngung“ wurde per Zufall aus den unterschiedlichen Düngestufen ausgewählt. Somit sind Varianten mit hoher und niedriger Düngung eingeflossen. Die Auswertung der Daten erfolgt nicht auf Parzellenebene sondern auf Variantenebene, also im Mittel der Wiederholungen. Da die Versuche im wesentlichen über mehrere Jahre liefen, wurde auch hier nach dem Zufallsprinzip von jedem Standort eine Variante „ohne Düngung“ und eine Variante „mit Düngung“ aus einem Jahr ausgewählt, da die Mehrfachaufführung eines gleichen Standortes zu einer Fallverzerrung führen würde (KÖHLER et al., 1995).

Abb. 3 gibt einen Überblick über die geographische Lage der Versuchsstandorte. 


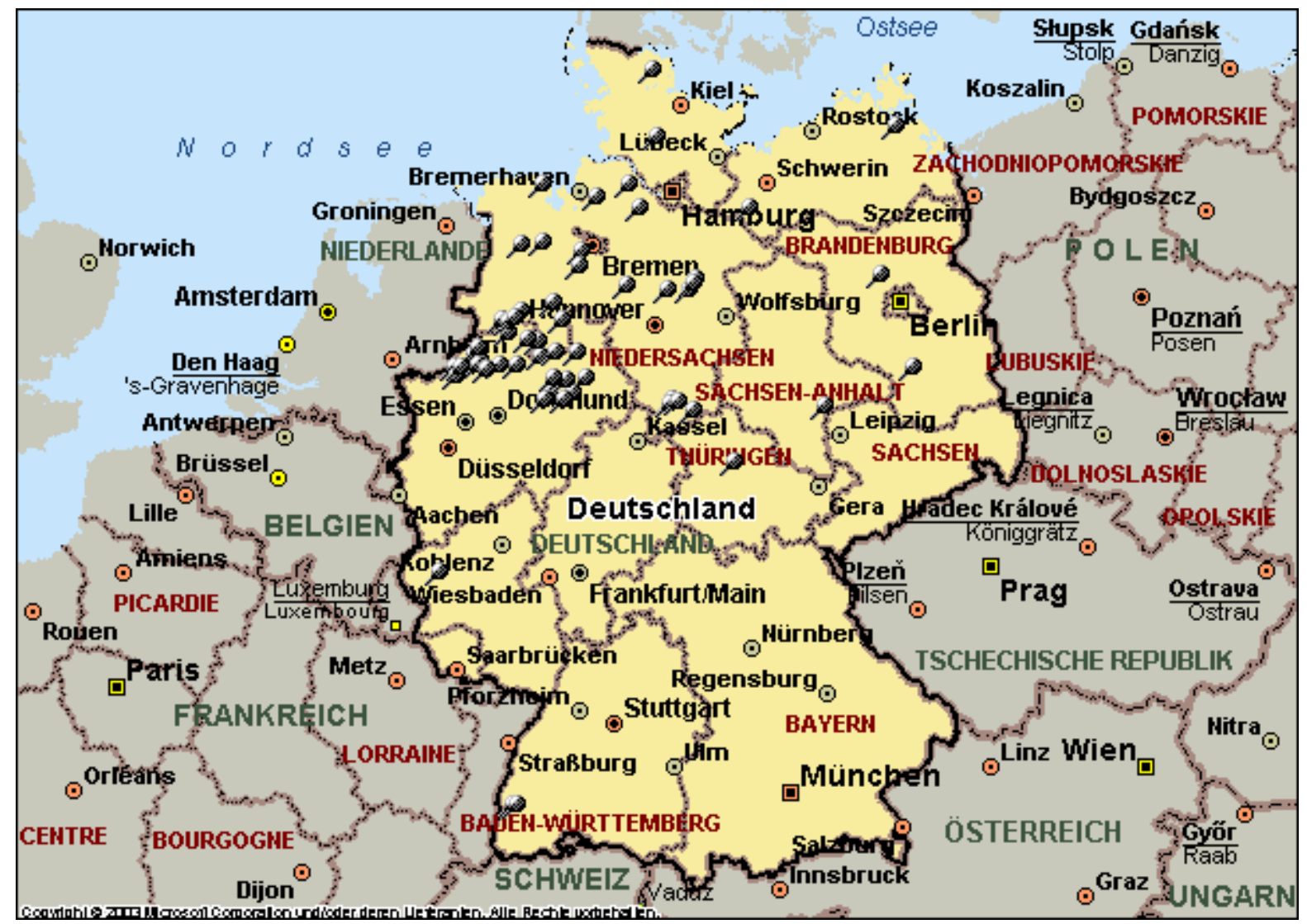

Abb. 3: Lage der Exaktversuchstandorte in Deutschland

\section{Variablen der verwendeten Daten}

Die Exaktversuchsdaten enthalten Angaben über die Herbst und Frühjahrs $\mathrm{N}_{\text {min- }}{ }^{-}$ Menge, den Ertrag, die Art und Höhe der Düngung, die Vorfrucht, die Vorvorfrucht, die Zwischenfrucht vor Mais, die Bodenart und das $\mathrm{C} / \mathrm{N}-$ Verhältnis in der Ackerkrume. Für alle Standorte wurden über den Deutschen Wetterdienst die täglichen Messwerte der Temperatur, des Niederschlages und der rel. Luftfeuchte der nächstgelegenen Klimastation beschafft. Die Struktur des Datensatzes ist in Tab. 4 dargestellt. In der Tabelle werden die Angaben der ausgewählten Varianten „ohne Düngung“ (78 Flächen) und „mit Düngung“ (135 Flächen) aufgezeigt. Auf Grund der Tatsache, dass nicht in jedem Versuch Varianten ohne Düngung angelegt worden sind, ist die Anzahl der Standorte unterschiedlich. 
Tab. 4: Zusammensetzung der Daten aus den Exaktversuchen nach der Randomisation (Datenkategorie 3)

\begin{tabular}{lcc}
\hline Variable & \multicolumn{2}{c}{ Anzahl } \\
& Ohne Düngung & Mit Düngung \\
\hline Frühjahrs $\mathrm{N}_{\text {min }}$ & 59 & 116 \\
${\text { Herbst } \mathrm{N}_{\min }}_{\text {Ertrag }}$ & 75 & 116 \\
Rohprotein & 77 & 126 \\
Düngung (Stickstoff) & 13 & 28 \\
(mineralisch + organisch) & 78 & 135 \\
C/N-Verhältnis & & \\
Humusgehalt & 22 & 46 \\
Grundbodenuntersuchung & 22 & 46 \\
(P2O5, K2O) & 53 & 76 \\
Grunddüngung & & \\
(P2O5, K2O) & 18 & 32 \\
pH-Wert & & \\
Ackerzahl & 66 & 110 \\
Bodenart & 68 & 121 \\
(klassifiziert) & 78 & 117 \\
Vorfrucht & & 71 \\
Vorvorfrucht & 76 & 62 \\
Zwischenfrucht & 31 & 133 \\
Tägliche Klimadaten & 20 & \\
\hline
\end{tabular}

\subsection{Methoden}

\section{Statistische Methoden}

Die statistische Datenauswertung wurde mit Hilfe des Softwarepakets SPSS (Version 11.5) durchgeführt. Zur Bestimmung von Ausreißern wurde auf das Programm WINSTAT zurückgegriffen, da die „Allgemeine 4-Sigma-Regel“ (SACHS, 2002) in SPSS nicht implementiert war. Mit dieser Regel wurden zunächst alle Ausreißer aufgezeigt, eine nachträgliche individuelle, inhaltliche Plausibilitätsprüfung entschied über einen Ausschluss. Hierbei sollte man immer berücksichtigen, dass durch das Herausnehmen von Daten auch wahre Informationen vernichtet werden können (IGLEWICZ und HOAGLIN, 1993). Das Verfahren der Ausreißerbestimmung fand nur in der analytischen Auswertung der Daten Anwendung. In der beschreibenden Auswertung wurden sämtliche Werte einbezogen. Die Anforderungen an die Prüfmerkmale nach Normalverteilung und Homogenität der Varianzen sind nach WEBSTER (2001) geprüft worden. Je nach Eingangsvoraussetzung der angewendeten statistischen Verfahren wurde eine Transformierung der Daten vorgenommen. Die teilweise unterschiedlichen Datenumfänge in den Auswertungen sind darin begründet, dass nicht von jeder 
Fläche alle Angaben vorhanden waren. Grundsätzlich wurde mit der maximal verfügbaren Flächenanzahl gerechnet.

Zur grafischen Darstellung von Ergebnissen wurden Histogramme und Boxplots verwendet. Die Histogramme stellen Häufigkeitsverteilungen in Form eines Balkendiagramms dar. Durch die schwarze geschwungene Linie (Normalverteilungskurve) in den Histogrammen wird die Normalverteilung dargestellt. Hierdurch lässt sich ein Eindruck davon gewinnen, ob die Daten normalverteilt sind. Durch einen Boxplot (Box-Whisker-Plot) werden Mediane, Perzentile, Ausreißer und Extremwerte von skalierten Variablen aufgezeigt. Den Aufbau eines Boxplots zeigt die Abb. 4.

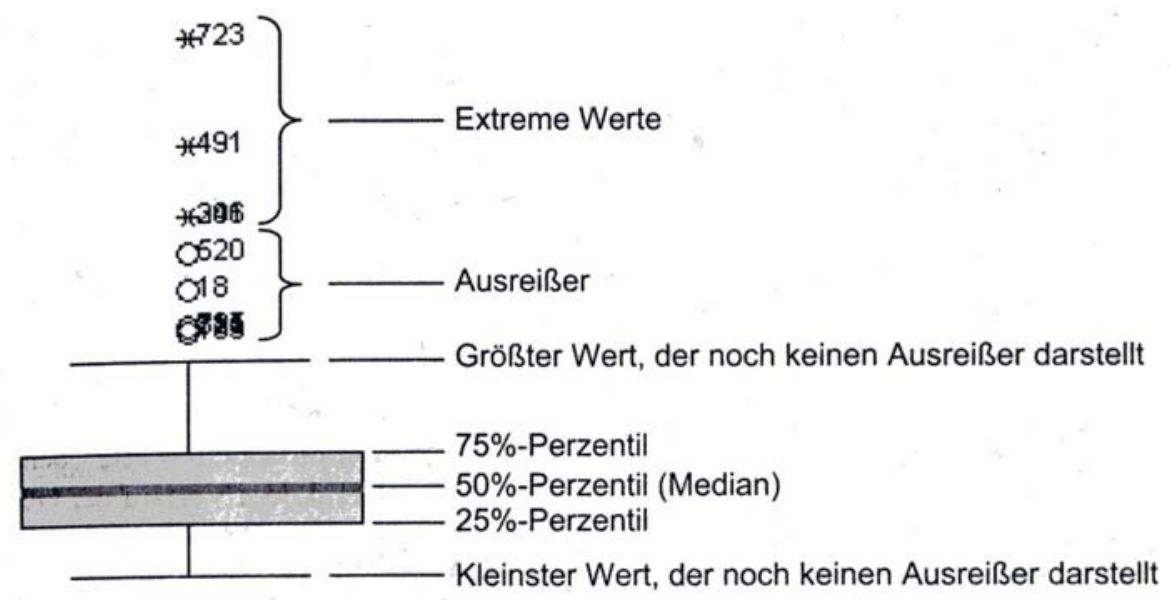

Abb. 4: Box-Wisker-Plot (BROSIUS, 2002)

Als struktur-entdeckendes Verfahren ist die Faktorenanalyse eingesetzt worden. Diese dient zur Bestimmung zugrunde liegender Faktoren, welche die Korrelationsmuster innerhalb eines Satzes beobachteter Variablen erklären. Die Anwendung erfolgt zur Identifikation weniger Faktoren, die den größten Teil der in den Variablen auftretenden Varianz erklären und zur Generierung von Hypothesen über kausale Mechanismen oder zur Sichtung von Variablen für sich anschließende Analysen führen (POSPESCHILL, 2001). Für die Durchführung einer ordnungsgemäßen Faktorenanalyse wurden der Bartlett-Test auf Sphärizität, das Kaiser-Meyer-Olkin-Maß (KMO) der Stichprobenadäquanz und der Measure of Sampling Adequacy (MSA) für die getesteten Variablen vorher untersucht. Die in die Faktorenanalyse eingeflossenen Variablen wurden anhand des KMO-Maßes und des MSA-Wertes ausgewählt. So wurde die Faktorenanalyse generell nur mit einem KMO-Maß von größer 0,5 durchgeführt. Variablen mit einem MSA-Wert von kleiner 
0,5 wurden dementsprechend ausgeschlossen (BROSIUS, 2002). Als Rotationsmethode diente die Varimax-Rotation. Zur Entscheidung, welche Faktoren relevant sind, wurde ein so genannter Screeplot gezeichnet. Es wurden nur Faktoren mit einem Eigenwert von größer 1 berücksichtigt. Zur Untersuchung des Einflusses der Faktoren (Komponenten) auf die abhängige Variable wurde die partielle Korrelation eingesetzt. Hierdurch war es möglich den Einfluss der weiteren Faktoren (Komponenten) auf die abhängige Variable zu berücksichtigen (BROSIUS, 2002).

Für den Vergleich von zwei Mittelwerten wurde bei normalverteilten Daten auf den tTest und bei nicht-normalverteilten Daten auf den Mann-Whitney-U-Test zurückgegriffen. Beim Vergleich mehrerer Mittelwerte (mehrere Teilgruppen der Grundgesamtheit) wurde bei den normalverteilten Daten die einfaktorielle Varianzanalyse (ANOVA) angewendet. Bei nicht-normalverteilten Daten wurde dementsprechend der Kruskal-Wallis Test, der die mittleren Rangwerte vergleicht, eingesetzt. Zur Prüfung von Zusammenhängen zweier normalverteilter Variablen wurden die Korrelationskoeffizienten nach Pearson und bei nicht-normalverteilten nach Kendall-Tau-B berechnet. Da bei der Berechung einer einfachen Korrelation der Fall eintreten kann, dass der Zusammenhang zwischen den beiden verwendeten Variablen durch einen nicht berücksichtigten Faktor verschleiert wird, wurde bei vorliegendem metrischen Skalenniveau auch die schrittweise multiple lineare Regressionsanalyse eingesetzt (BÜHL und ZÖFEL, 2002). Diese berechnet die Beziehung jeder einzelnen unabhängigen Variablen zur abhängigen Variable unter der Bedingung, dass die anderen unabhängigen Variablen konstant sind (LAMPRECHT, 1999). Vor der Durchführung einer multiplen Regression wurde zum Aufspüren einer Autokorrelation der Durbin-Watson-Test durchgeführt. Werte zwischen 1,5 und 2,5 wurden als akzeptabel angesehen (BROSIUS, 2002). Zur Kollinearitätprüfung wurden die Toleranz und der Varianzinflationsfaktor (VIF) ermittelt. Kollinearität kann ab einem Toleranzwert von $<0,1$ und einem VIF Wert von $>10$ auftreten. Auftretender Kollinearität kann durch das Entfernen von Variablen aus der Regressionsgleichung begegnet werden (BACKHAUS et al. 2000). 


\section{Variablen - Neuberechnung und Kategorisierungsmethoden}

In diesem Abschnitt werden Berechnungen von Variablen und Kategorisierungen von Daten näher erläutert.

\section{Organische Düngung}

Die Anrechenbarkeit der organischen Düngemittel wurde über die verschiedenen Datensätze angeglichen. So wurde die Stickstoff-Anrechenbarkeit für die organischen Düngemittel aus dem Datensatz der Wasserschutzgebietsberatung der Landwirtschaftskammer Weser-Ems auf die anderen Daten übertragen. Gülle wurde zu 80\% und Mist zu 40\% angerechnet.

\section{N-Entzug}

Da von sehr wenigen Flächen der wirkliche N-Gehalt (mit dem Faktor 6,25) aus dem Rohproteingehalt in der Pflanze bestimmt werden konnte, wurde von allen Flächen der N-Gehalt bzw. der Nährstoffentzug anhand der Richtwerte von ZSCHEISCHLER et al. (1990) berechnet. Es wurde davon ausgegangen, dass Silomais je dt Trockenmasse 1,3 kg N und Körnermais (ohne Stroh) je dt Trockenmasse 1,74 kg N entzieht. Hierdurch soll eine bessere Vergleichbarkeit mit den anderen Datenkategorien erzielt werden.

\section{N-Saldo}

Der N-Saldo wurde aus dem berechneten N-Entzug der geernteten Biomasse und der ausgebrachten Düngermenge berechnet (Düngung - N-Entzug).

\section{Trockenmasseertrag}

Da der Trockenmasseertrag nicht generell vorlag, wurde dieser zum Teil aus dem Frischmasseertrag berechnet. Überwiegend konnte die Umrechnung anhand der bei der Ernte vorliegenden Trockensubstanzgehalte vorgenommen werden. Die in wenigen Fällen nicht vorliegenden Trockensubstanzgehalte wurden durch festgelegte Werte berechnet. Bei der Ernte des Silomaises wurde von einem Trockensubstanzgehalt von 32\% und beim Körnermais von 68\% ausgegangen. Der Trockensubstanzgehalt des Silomaises entspricht der Empfehlung des MAISKOMITEE (1999), beim Körnermais wurde ein Kompromiss aus der 
Empfehlung des MAISKOMITEE (1999) und den gemessenen Daten der LANDWIRTSCHAFTKAMMER WESTFALEN-LIPPE (2003) gebildet.

\section{Zwischenfruchtkategorisierung}

Da die Angaben zur Zwischenfrucht teilweise kategorisiert vorlagen, sind die anderen Daten dementsprechend angepasst worden. Die Angaben zur Zwischenfrucht wurden in die Kategorien „Brache“, „Leguminose“ und „Nicht Leguminose“ eingeteilt. In die Kategorie „Brache“ wurden alle Flächen aufgenommen, von denen bekannt war, dass keine Zwischenfrucht vorhanden war. Die Kategorie „Leguminose“ enthält nur leguminose Zwischenfrüchte. Hingegen enthält die Kategorie „Nicht Leguminose“ nur Zwischenfrüchte, die keine Leguminose waren.

\section{Bodenartenkategorisierung}

Da die Bodenarten von einigen Institutionen gruppiert überliefert worden sind, wurde diese Gruppierung übernommen. Die Bodenarten wurden nach dem Tonanteil gruppiert. Es wurden die drei Kategorien „leicht“, „mittel“ und „schwer" gebildet. Genaue Angaben zur Aufteilung der Bodenarten sind dem Anhang 6 zu entnehmen.

\section{Gesamtkohlenstoffgehalt des Bodens ( $\left.\mathrm{C}_{t}\right)$}

In den Untersuchungen ist $\mathrm{C}_{\mathrm{t}}=\mathrm{C}_{\text {org }}$ gesetzt worden. Dies geschah vor dem Hintergrund, dass nicht alle Versuchsansteller die Kohlenstoffgehalte der Böden nach Gesamtkohlenstoff oder organischen Kohlenstoff unterschieden haben. Nach

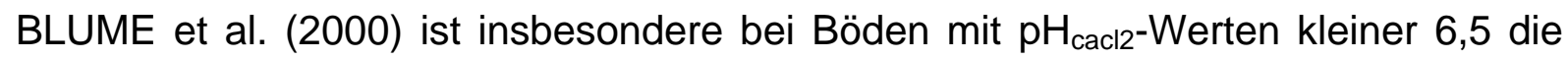
Anwesenheit von Carbonaten generell unwahrscheinlich, daher ist diese Gleichsetzung vertretbar. 


\section{Ergebnisse}

\subsection{Daten aus der Wasserschutzgebietsberatung (Datenkategorie 1)}

\subsubsection{Variabilität der untersuchten Merkmale}

Für die Auswertung der Daten aus den Wasserschutzgebieten stehen je nach Merkmal von 173 bis 500 Werte zur Verfügung. Zunächst soll mit Hilfe der beschreibenden Statistik die Variabilität einiger Merkmale dargestellt werden. Abb. 5 zeigt die Häufigkeitsverteilung der Herbst $\mathrm{N}_{\min }$-Gehalte. Im Mittel wurden $\mathrm{N}_{\text {min- }}$ Mengen von $77 \mathrm{~kg} \mathrm{~N} / \mathrm{ha}$ im Bodenprofil 0 bis $90 \mathrm{~cm}$ festgestellt. Die Beprobung der Herbst $N_{\text {min }}$-Menge fand hauptsächlich im Zeitraum von Ende Oktober bis Mitte November statt. Inwieweit die einzelnen Jahre einen Effekt auf den Herbst $\mathrm{N}_{\text {min}}{ }^{-}$ Gehalt haben, wird im Anhang gezeigt (Anhang 1). Hier wurden die Jahre ausgewählt, in denen die höchsten Beprobungsdichten vorhanden waren. Unter den Häufigkeitsverteilungen der Herbst $\mathrm{N}_{\min }$-Gehalte hebt sich besonders das Jahr 1998 mit niedrigen Herbst $\mathrm{N}_{\text {min }}$-Mengen hervor. Durch den Einsatz des Kruskal-Wallis Tests konnten Jahreseffekte mit einem Signifikanzniveau von <0,001 bestätigt werden. Inwieweit die generell niedrigeren Herbst $\mathrm{N}_{\min }$-Gehalte sich hier durch die sehr starken Niederschläge im Herbst des Jahres 1998 erklären lassen, wird in einem späteren Abschnitt untersucht.

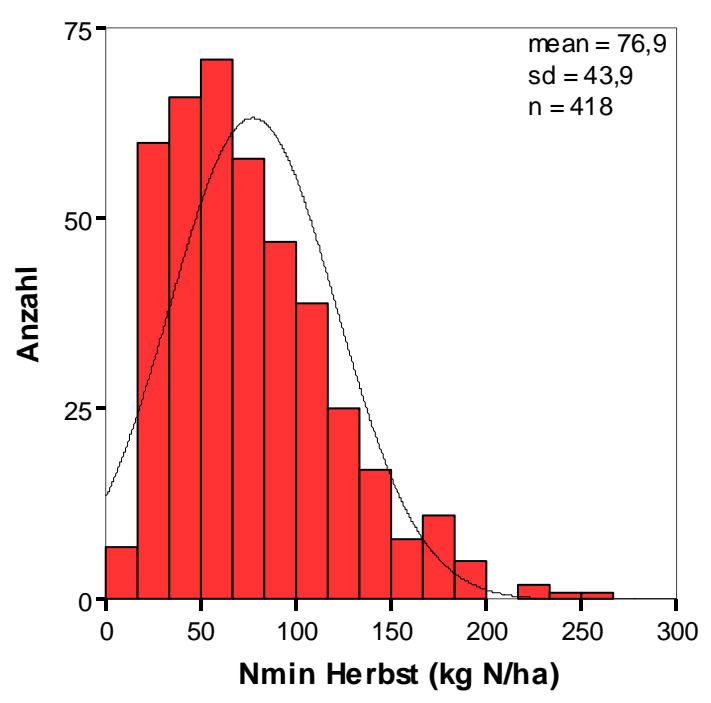

Abb. 5: Häufigkeitsverteilung der Herbst $\mathrm{N}_{\min }$-Gehalte (0-90cm / Datenkategorie 1) 
Die Frühjahrs $\mathrm{N}_{\min }$-Gehalte $(0-90 \mathrm{~cm})$ wurden über einen relativ weiten Beprobungszeitraum ermittelt. So lagen die meisten Beprobungstermine zwischen März und Ende April oder zwischen Mitte Mai und Mitte Juni. Aus diesem Grund sollte bei der Betrachtung des Histogrammes (Abb. 6) der zeitliche Einfluss auf die $\mathrm{N}_{\text {min }}$-Menge berücksichtigt werden. Innerhalb des Pools der Frühjahrs $\mathrm{N}_{\min }$-Gehalte wurden 167 Flächen vor dem 1. Mai mit $52 \mathrm{~kg} \mathrm{~N} \mathrm{~N}_{\text {min }} /$ ha und 63 Flächen ab dem 1. Mai mit $161 \mathrm{~kg} \mathrm{~N} \mathrm{~N}_{\min } /$ ha im Mittel beprobt (Anhang 2).

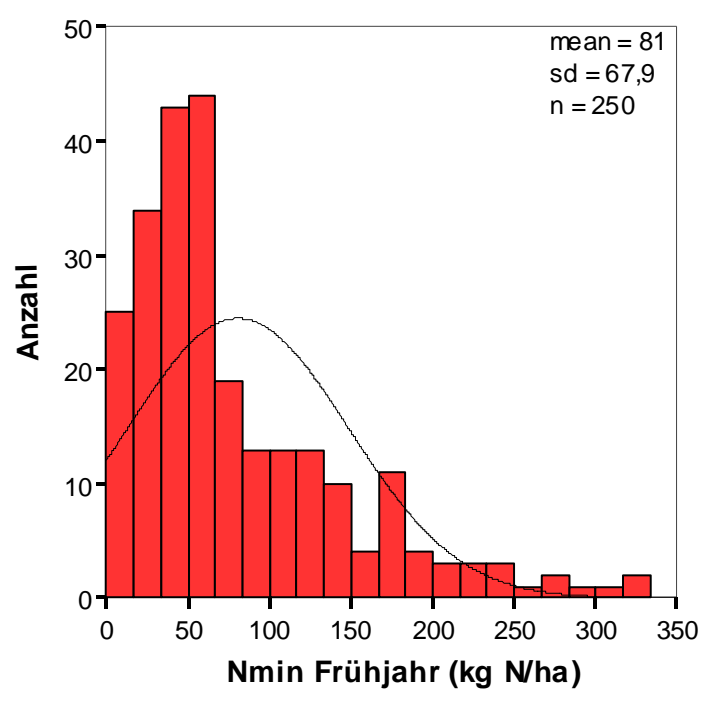

Abb. 6: Häufigkeitsverteilung der Frühjahrs $\mathrm{N}_{\min }$-Gehalte (0-90cm / Datenkategorie 1)

Die ausgebrachte Düngermenge (mineralisch und organisch) lag im Mittel bei $196 \mathrm{~kg}$ $\mathrm{N} / \mathrm{ha}$ (Abb. 7). Bei den Angaben zu den hier verwendeten Mengen handelt es sich um Schätzungen der Wasserschutzgebietsberater im Zuge der Düngeplanung. Nur die Daten der Landwirtschaftskammer Schleswig-Holstein sind exakt auf den Betrieben erhoben worden. Die applizierten organischen Düngemittel waren Gülle (85,2\%), Rinderfestmist (9,5\%), Hähnchenmist (3,7\%), Hühnertrockenkot $(0,9 \%)$, Klärschlamm $(0,4 \%)$ und Jauche $(0,2 \%)$. 


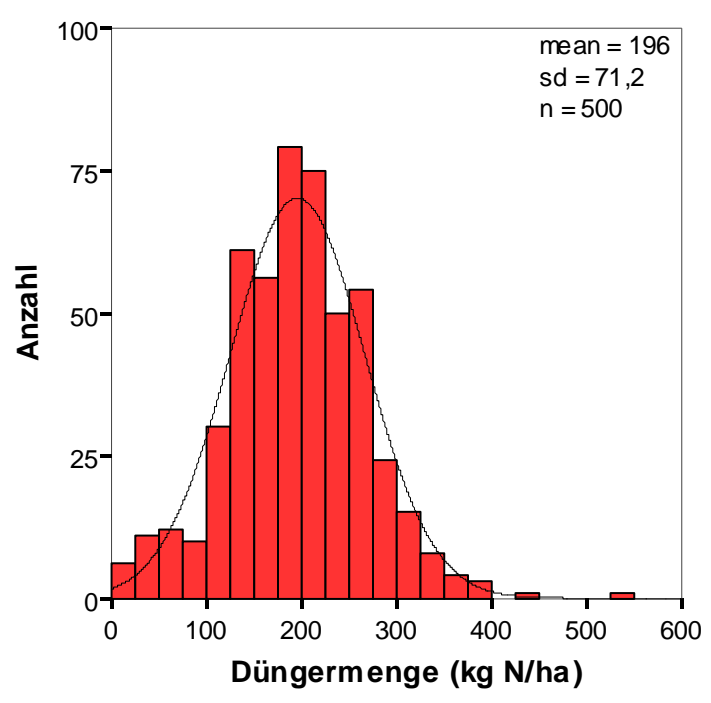

Abb. 7: Häufigkeitsverteilung der aufgewendeten mineralischen und organischen Düngermenge in kg N/ha (Datenkategorie 1)

Die geschätzten Silo- und Körnermaiserträge werden im Anhang dargestellt (Anhang 3). Insgesamt wurde von 461 Flächen der Ertrag durch Schätzung ermittelt. 322 Flächen waren hiervon mit Silomais bestellt und brachten im Mittel einen geschätzten Frischmasseertrag von $444 \mathrm{dt} / \mathrm{ha}$. Des Weiteren sind von 139 Flächen die Körnermaiserträge bekannt. Hier wurden nach der Schätzung im Mittel $85 \mathrm{dt} / \mathrm{ha}$ bei einer Trockensubstanz von 86\% geerntet.

Von 461 Flächen wurden auf Basis der Ertragschätzungen N-Salden berechnet. Diese lagen im Mittel über die Jahre bei etwa $39 \mathrm{~kg} \mathrm{~N} / \mathrm{ha}$.

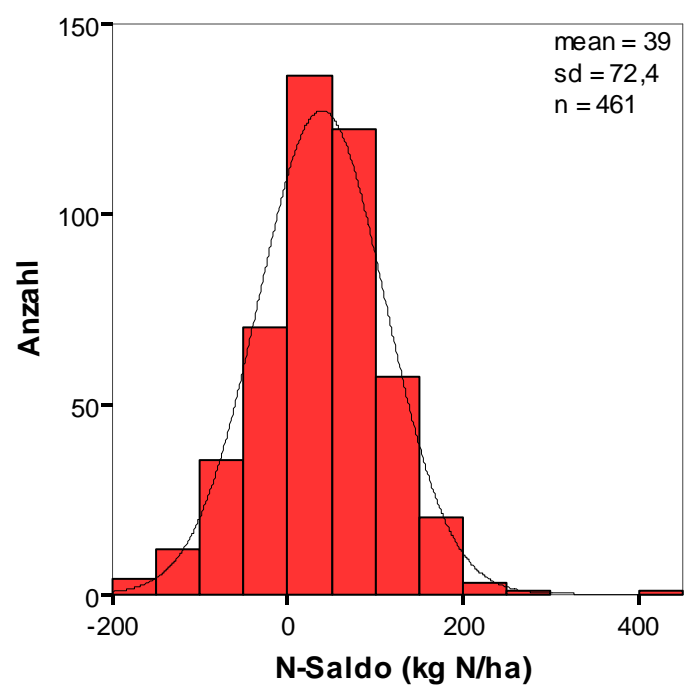

Abb. 8: Häufigkeitsverteilung der geschätzten N-Salden (kg N/ha / Datenkategorie 1) 
Die Vorfrüchte der Maisflächen sind von 444 Flächen bekannt (Anhang 4). Auf 65\% dieser Flächen standen Wintergerste (124 Flächen), Silomais (108 Flächen) und Winterroggen (57 Flächen) als Vorfrucht vor dem Maisanbau. Angaben zum Zwischenfruchtanbau vor dem Mais liegen von 433 Flächen vor (Anhang 5). So lagen $19,6 \%$ der Flächen vor dem Anbau von Mais brach. Als Zwischenfrucht dominierte der über Winter abfrierende Senf mit einem Anteil von nahezu 44\%.

\section{Daten zu der Betriebsstruktur}

Daten zur Struktur der Betriebe, auf deren Flächen die Untersuchungen durchgeführt wurden, stehen für die hier vorgenommene Auswertung nur in geringem Umfang zur Verfügung. Dies ist $u$ a. darauf zurückzuführen, dass die Wasserschutzgebietsberatung einen Schwerpunkt auf die Düngung und Nutzung der bewirtschafteten Flächen legt. Es kann vermutet werden, dass Angaben zum betrieblichen Viehbesatz in enger Beziehung zur Praxis der organischen Düngung zu Mais stehen. Aus diesem Grund werden die Betriebsstrukturdaten - soweit vorhanden - in die Auswertung einbezogen. Dabei ist jedoch zu berücksichtigen, dass diese Auswertung erst im Zusammenhang mit weiteren betriebsbezogenen Daten wie beispielsweise zum Fruchtartenverhältnis und zur Stall-Feld-Entfernung ein vollständiges Bild ergeben. Insgesamt 191 Flächen konnte eine Angabe zum Viehbesatz des bewirtschaftenden Betriebes zugeordnet werden. Hier zeigt sich wie erwartet, dass viele der Maisflächen in den veredelungsstarken Regionen liegen. So ist bei $69 \%$ der zur Verfügung gestellten Flächen, von denen der Viehbesatz bekannt ist, ein Viehbesatz von über 2 Großvieheinheiten je Hektar anzutreffen. 


\section{Standortbezogene Daten und deren Struktur}

Die dominierende Bodenart in den Daten aus der Wasserschutzgebietsberatung ist der Sandboden. Die Bodenarten nach Reichsbodenschätzung wurden nach einem festen Schema gruppiert (Anhang 6).

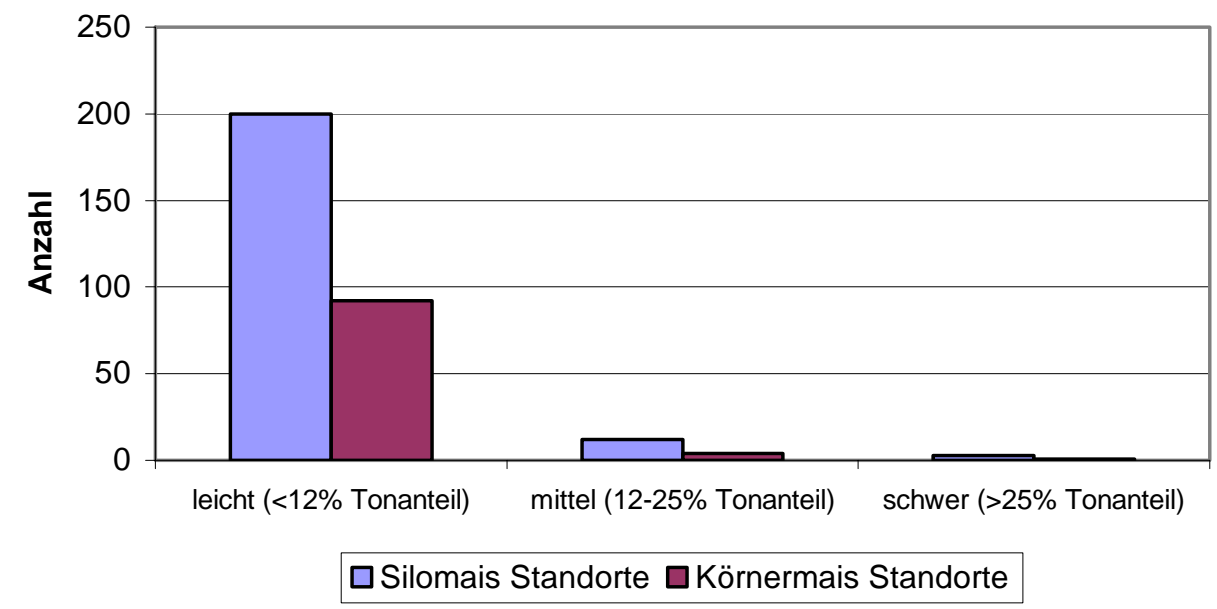

Abb. 9: Häufigkeitsverteilung der Silo- und Körnermaisflächen nach gruppierten Bodenarten (Datenkategorie 1)

Als weiteres quantitatives Bodenmerkmal liegt das C/N-Verhältnis für 173 Flächen vor (Abb. 10). Von 100 dieser Flächen wurden zur Bestimmung der $\mathrm{C}_{t^{-}}$und $\mathrm{N}_{\mathrm{t}}-$ Werte Bodenproben nachträglich erhoben. Im Mittel der Flächen liegt das $\mathrm{C} / \mathrm{N}-$ Verhältnis bei 14. Für die drei Datenherkünfte beträgt das C/N-Verhältnis 12,3 (Weser-ElbeDreieck), 14,5 (Schleswig-Holstein) und 14,1 (Weser-Ems).

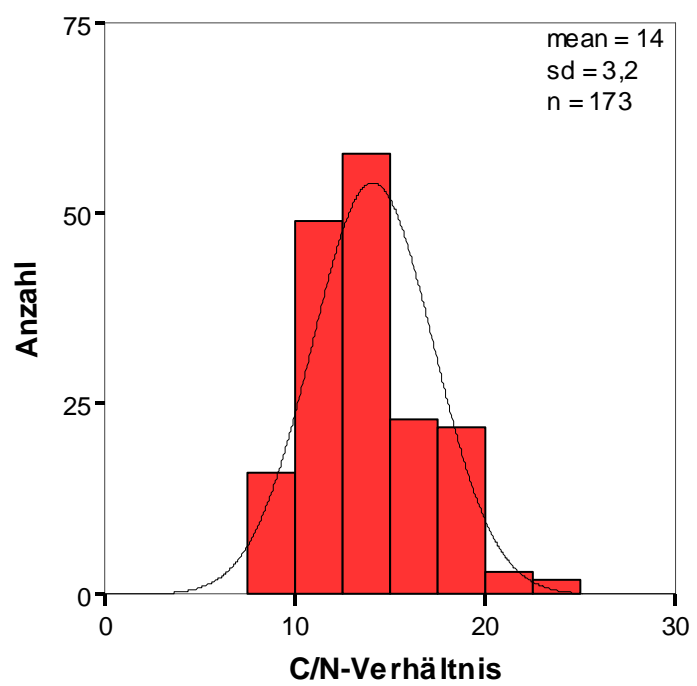

Abb. 10: Häufigkeitsverteilung der C/N-Verhältnisse (Datenkategorie 1) 


\subsubsection{Analytische Statistik}

Für die weitere Analyse der Struktur der Daten werden multivariate Verfahren angewendet. Mit Hilfe des faktoranalytischen Ansatzes sollte versucht werden, Hintergrundvariablen zu extrahieren, welche im Kern standörtliche bzw. betriebsstrukturelle Charakteristika beinhalten würden. Doch einige Voraussetzungen für eine ordnungsgemäße Faktorenanalyse können nicht erreicht werden. So erfüllt für die getesteten Variablen zwar der Bartlett-Test auf Sphärizität mit einem Signifikanzniveau von <0,001 die Voraussetzungen für die Durchführung einer Faktorenanalyse, nicht jedoch das Kaiser-Meyer-Olkin-Maß (KMO) der Stichprobenadäquanz und der Measure of Sampling Adequacy (MSA). Aus diesem Grund wird für diese Datenkategorie keine Faktorenanalyse durchgeführt.

Nachdem bisher struktur-entdeckende Verfahren eingesetzt wurden, sollen in den anschließenden Diagrammen ausgewählte Variablen gegenüber gestellt werden. Das in Abb. 11 dargestellte Verhältnis zeigt, dass sowohl die ausgebrachte Düngermenge als auch der Herbst $\mathrm{N}_{\min }$-Gehalt stark streut, und dass es keinen Zusammenhang zwischen diesen beiden Merkmalen gibt. Die mittlere Düngermenge in Abb. 11 liegt bei $190 \mathrm{~kg} \mathrm{N/ha}$ und der mittlere Herbst $N_{\min }$-Gehalt bei $76 \mathrm{~kg} \mathrm{~N}$ min $/$ ha.

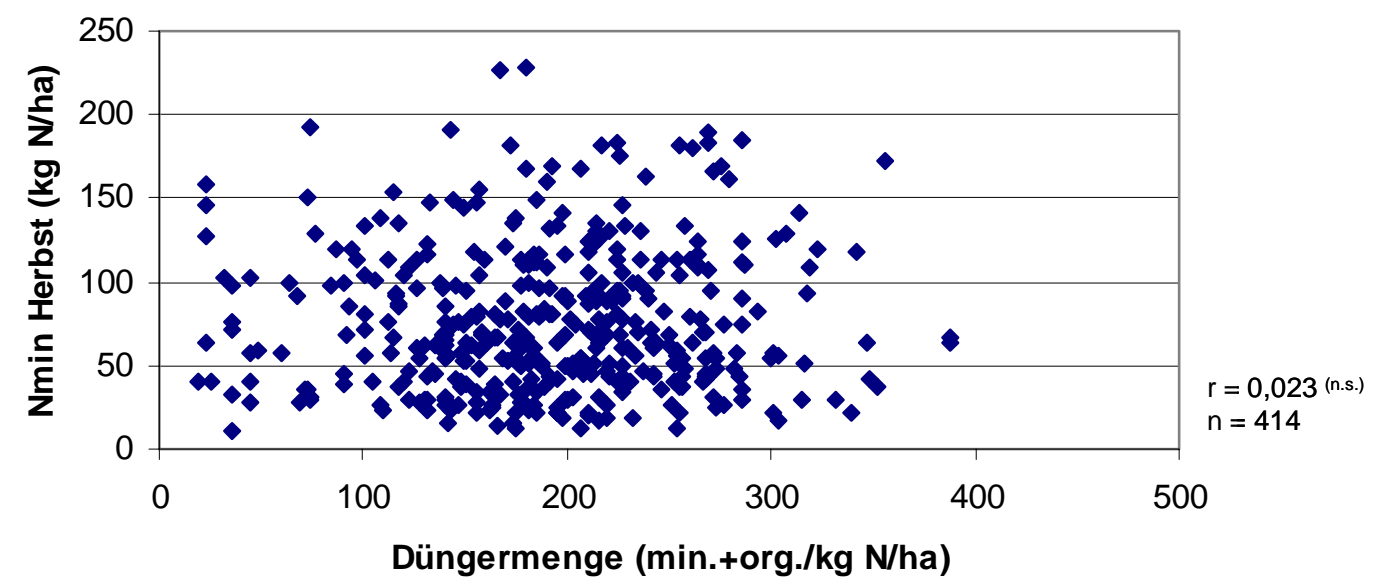

Abb. 11: Herbst $\mathrm{N}_{\min }$-Gehalte im Verhältnis zur ausgebrachten mineralischen und organischen Düngermenge (Korrelation nach Pearson / Datenkategorie 1)

Eine weitere Differenzierung der Abb. 11 nach der Nutzungsart bringt keine weiteren Erkenntnisse. So unterscheiden sich die mittlere Düngermenge zwischen Körnerund Silomais um ca. 14 kg N/ha. Der Mittelwert für den Silomais (291 Flächen) liegt bei $194 \mathrm{~kg} \mathrm{~N} / \mathrm{ha}$ und der für den Körnermais (120 Flächen) bei $180 \mathrm{~kg} \mathrm{~N} / \mathrm{ha}$. Die $\mathrm{N}_{\text {min- }}$ 
Gehalte betragen im Falle des Silomaises $75 \mathrm{~kg} \quad \mathrm{~N}_{\min } / \mathrm{ha}$ und bei der Körnermaisnutzung $78 \mathrm{~kg} \mathrm{~N} \mathrm{~min}_{\text {ma }} / \mathrm{ha}$. Auf den fehlenden drei Flächen, von denen die Düngung und der Herbst $\mathrm{N}_{\min }$-Gehalt bekannt sind (insgesamt 414 Flächen), wird der Mais einer anderen Nutzung zugeführt.

In der Tab. 5 werden die Daten nach ihren Herkunftsregionen untergliedert. Auf Grund der stark unterschiedlichen Gruppengrößen werden die Fallgruppen mittels des Kruskal-Wallis Tests anhand der mittleren Ränge untersucht. Jedem Variablenwert wird dabei ein Rangwert gemäß seiner Position in der Rangordnung zugewiesen, anschließend werden die durchschnittlichen Rangwerte getrennt für die Kategorien errechnet. Hier zeigt sich, dass die Herbst $\mathrm{N}_{\min }$-Menge zwischen den Datenherkünften nicht signifikant unterschiedlich ist. Hingegen unterscheiden sich die Düngermengen je nach Datenherkunft mit einem Signifikanzniveau von $<0,001$.

Tab. 5: Mittelwert und mittlerer Rang (Kruskal-Wallis Test) der Herbst $\mathrm{N}_{\min }-$ Mengen und der $\mathrm{N}$ Düngung (Datenkategorie 1)

\begin{tabular}{llccc}
\hline & Datenherkunft & $\mathbf{n}$ & Mittelwert & Mittlerer Rang \\
\hline $\mathrm{N}_{\text {min }}$-Gehalt & Weser-Ems & 390 & 77 & 211,8 \\
$(\mathrm{~kg} \mathrm{~N} / \mathrm{ha})$ & Weser-Elbe-Dreieck & 13 & 61 & 175,1 \\
& Schleswig-Holstein & 13 & 51 & 142,2 \\
\hline org. + min. & Weser-Ems & 464 & 198 & 259,3 \\
Düngermenge & Weser-Elbe-Dreieck & 13 & 153 & 140,7 \\
(kg N/ha) & Schleswig-Holstein & 21 & 137 & 100,7 \\
\hline
\end{tabular}

Um den Einfluss der Bodenart auf den Zusammenhang zwischen der Düngermenge und der Herbst $\mathrm{N}_{\min }$-Menge zu prüfen, werden Korrelationen nach Pearson für die häufigsten Bodenarten, die 92\% aller Flächen umfassten, berechnet (Tab. 6). Die berechneten Korrelationskoeffizienten zeigen keine signifikanten Zusammenhänge auf. Lediglich auf lehmigen Sand ist eine Tendenz zu etwas höheren Korrelationskoeffizienten zwischen der Düngermenge und dem Herbst $\mathrm{N}_{\min }$-Gehalt zu erkennen. Doch auch diese ist mit einem Signifikanzniveau von 0,064 nicht abzusichern.

Tab. 6: Korrelation (Pearson) zwischen N-Düngermenge (mineralisch/organisch) und dem Herbst $\mathrm{N}_{\min }$-Gehalt (0-90cm / Datenkategorie 1)

\begin{tabular}{lcc}
\hline Bodenart & Korrelationskoeffizient (r) & Anzahl (n) \\
\hline Sand & $0,012^{\text {(n.s.) }}$ & 155 \\
anlehmiger Sand & $0,030^{(\text {n.s. }}$ & 45 \\
lehmiger Sand & $0,273^{\text {(n.s.) }}$ & 47 \\
Sand / anlehmiger Sand / & $0,067^{\text {(n.s.) }}$ & 247 \\
lehmiger Sand (zusammen) & & \\
\hline
\end{tabular}


Die Bodenart hatte einen Einfluss auf die vom Landwirt ausgebrachte Düngermenge. Auf den Sandböden wurde im Mittel $183 \mathrm{~kg} \mathrm{~N} / \mathrm{ha}$ ausgebracht, auf den anlehmigen Sanden und den lehmigen Sanden $207 \mathrm{~kg} \mathrm{~N} / \mathrm{ha}$. Dieser Unterschied ist signifikant (Kruskal-Wallis Test $/ p=0,019$ ). Der Zusammenhang zwischen der applizierten mineralischen Düngermenge und dem Herbst $\mathrm{N}_{\min }$-Gehalt wird in Abb. 12 gezeigt. In diesem Streudiagramm lässt sich kein Trend erkennen. Das Streudiagramm zeigt eine starke Variation der Herbst $\mathrm{N}_{\min }$-Menge bei einer ausgebrachten Düngermenge von weniger als $50 \mathrm{~kg} \mathrm{~N} / \mathrm{ha}$. Im Mittel werden $51 \mathrm{~kg} \mathrm{~N} / \mathrm{ha}$ mineralisch gedüngt. Das führt zu einem mittleren Herbst $\mathrm{N}_{\text {min }}$-Gehalt von $76 \mathrm{~kg} \mathrm{~N} / \mathrm{ha}$. Die große Anzahl von Flächen, die mit einer mineralischen Düngermenge von weniger als $50 \mathrm{~kg} \mathrm{~N} / \mathrm{ha}$ gedüngt wurden, lässt den Schluss zu, dass die mineralische Düngung durch organische ergänzt wurde.

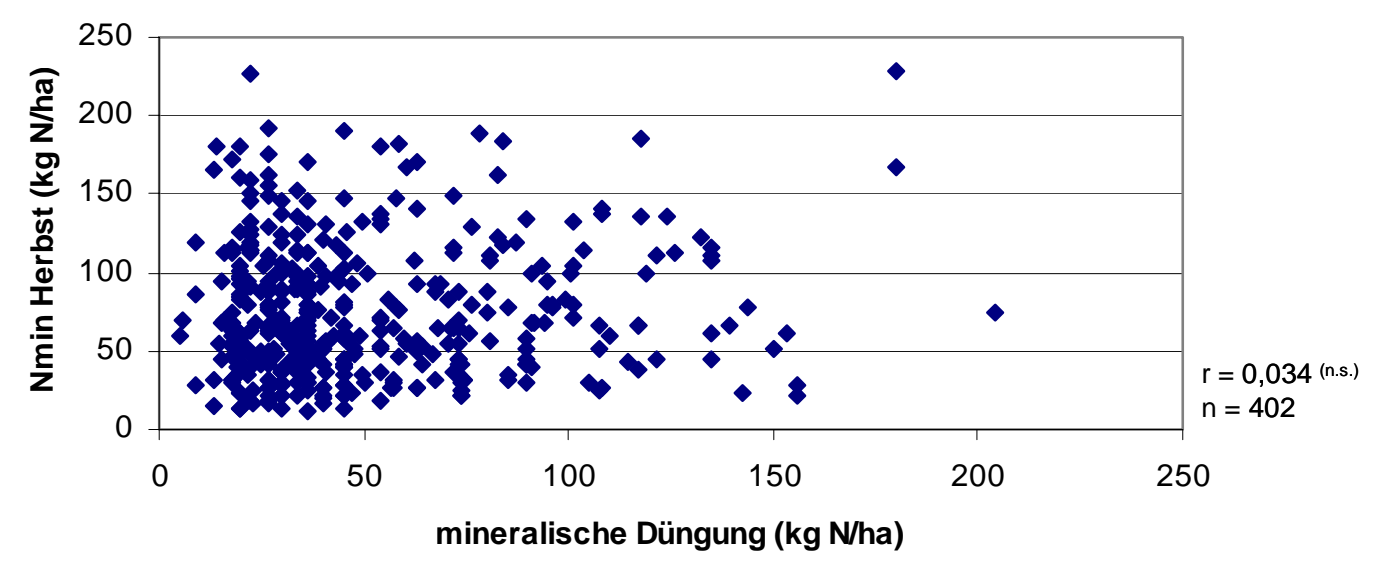

Abb. 12: Herbst $\mathrm{N}_{\min }$-Gehalte im Verhältnis zur ausgebrachten mineralischen Düngermenge (Korrelation nach Pearson / Datenkategorie 1)

Inwieweit Betriebe mit einem höheren Viehbesatz weniger mineralischen Dünger einsetzen, lässt sich bei den nicht normalverteilten Daten mittels des U-Tests nach Mann und Whitney $(p=0,085)$ nicht bestätigen (Abb. 13). Somit besteht offenbar die Tendenz, auf eine mineralische Ergänzungsdüngung auch bei stärkerem Einsatz organischer Dünger nicht adäquat zu verzichten. 


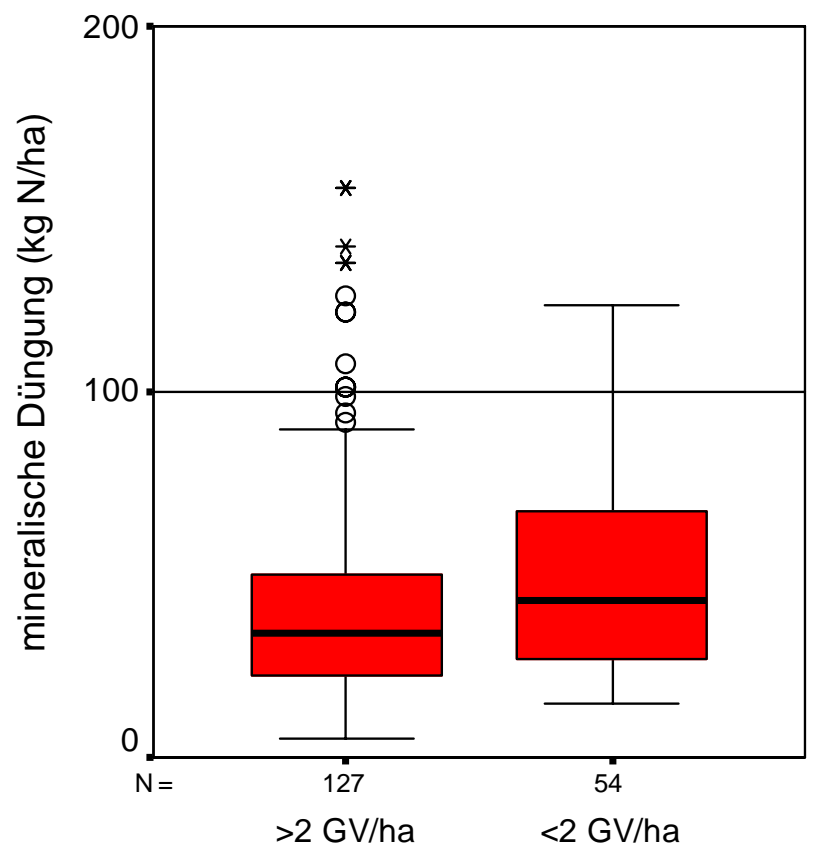

Abb. 13: Mittlere ausgebrachte mineralische Düngermenge ( $\mathrm{kg} \mathrm{N} / \mathrm{ha}$ ) in Abhängigkeit vom Viehbesatz der Betriebe (< oder > 2 GV/ha / Datenkategorie 1)

Dass kein Zusammenhang zwischen der Menge an organischem Dünger und der Herbst $\mathrm{N}_{\min }$-Menge im Boden besteht, zeigt Abb. 14. Der Mittelwert an ausgebrachter organischer Düngermenge liegt bei $162 \mathrm{~kg} \mathrm{~N} / \mathrm{ha}$ und der korrespondierende Herbst $\mathrm{N}_{\min }$-Gehalt bei $76 \mathrm{~kg} \mathrm{~N}$ min $/$ ha.

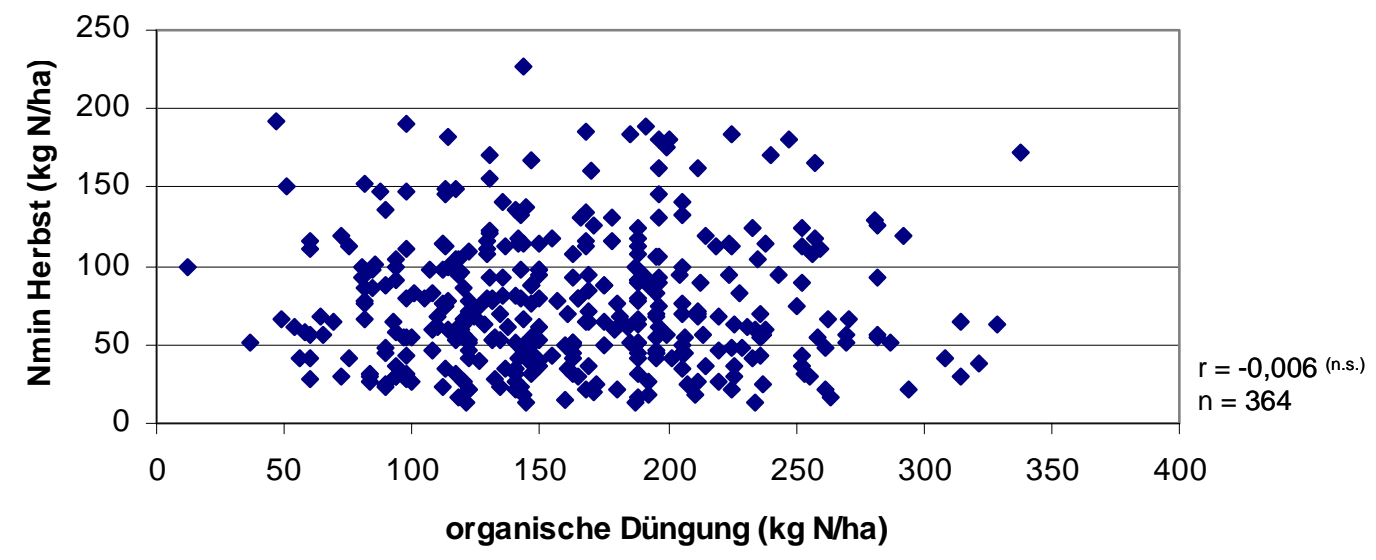

Abb. 14: Herbst $\mathrm{N}_{\text {min }}$-Gehalte im Verhältnis zur ausgebrachten organischen Düngermenge (Korrelation nach Pearson / Datenkategorie 1) 
Durch den Viehbesatz wird die ausgebrachte organische Düngermenge beeinflusst (Abb. 15). Bei einem Vergleich der beiden Mittelwerte ist auf Grund der normalverteilten Daten der $\mathrm{t}$-Test angewendet worden. Dieser weist einen signifikanten Unterschied $(p=0,032)$ der ausgebrachten organischen Düngermenge zwischen den Viehbesatzdichten auf. Betriebe mit geringerem Viehbesatz (<2GV/ha) düngen weniger organischen Dünger als Betriebe mit höherem Viehbesatz $(>2 \mathrm{GV} / \mathrm{ha})$.

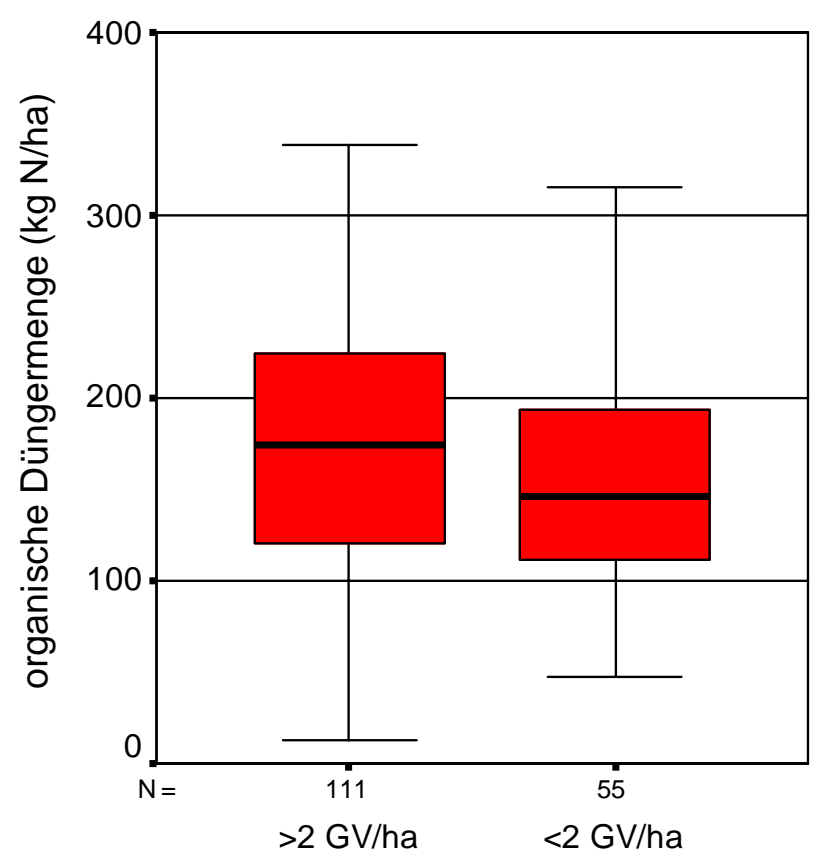

Abb. 15: Ausgebrachte organische Düngermenge (kg N/ha) in Abhängigkeit vom Viehbesatz der Betriebe (< oder > 2 GV/ha / Datenkategorie 1)

Zwischen dem Viehbesatz und den $\mathrm{N}_{\min }$-Mengen im Frühjahr und Herbst ist kein Zusammenhang festzustellen.

Auf den Flächen, die nur mineralisch (53 Flächen) oder organisch (16 Flächen) gedüngt wurden, zeigen sich ebenfalls keine korrelativen Beziehungen zum Herbst $\mathrm{N}_{\text {min- }}$-Gehalt. Die weiteren 431 Flächen wurden kombiniert gedüngt (Anhang 20).

Zwischen dem N-Saldo und der Herbst $\mathrm{N}_{\text {min }}$-Menge besteht keine Beziehung. Dieses wird anhand eines Streudiagrammes in Abb. 16 für 384 Flächen dargestellt. Der NSaldo beträgt im Mittel $33 \mathrm{~kg} \mathrm{~N} / \mathrm{ha}$ und die Herbst $\mathrm{N}_{\min }-$ Menge $77 \mathrm{~kg} \mathrm{~N}$ min $/$ ha. 


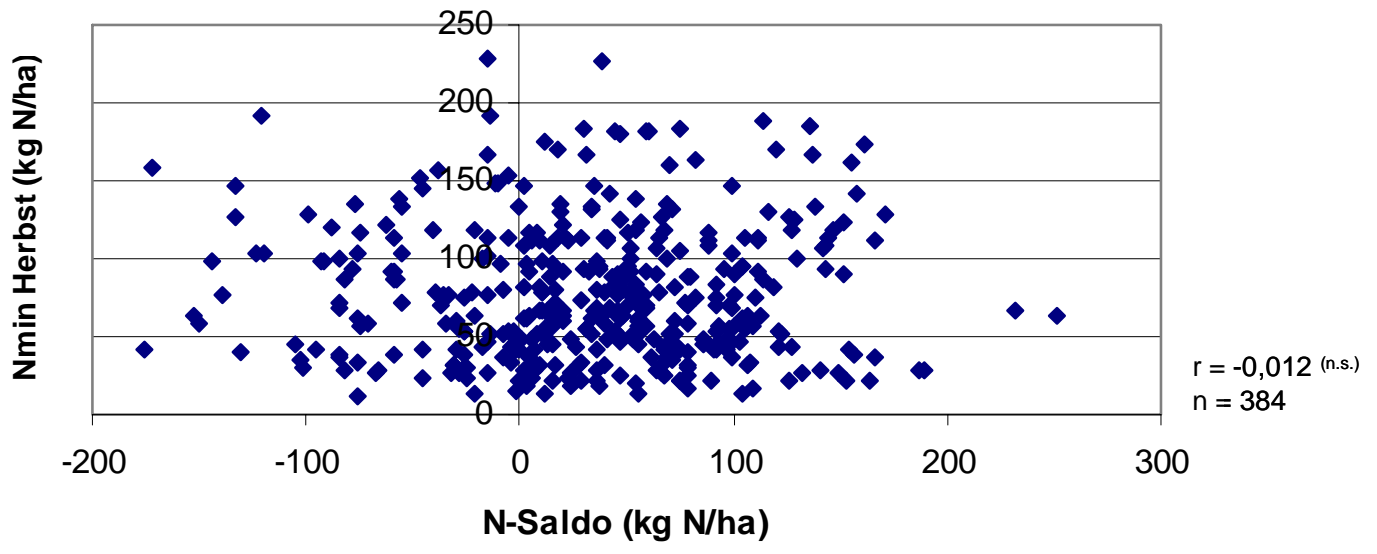

Abb. 16: Herbst $\mathrm{N}_{\min }$-Gehalte von Silo- und Körnermaisflächen in Abhängigkeit vom N-Saldo (Korrelation nach Pearson I Datenkategorie 1)

Auch bei einer Klassifizierung der Daten (Tab. 7) nach Standort und Nutzungsart kann kein signifikanter Zusammenhang zwischen dem N-Saldo und den Herbst $\mathrm{N}_{\text {min }}{ }^{-}$ Mengen festgestellt werden.

Tab. 7: Korrelation (Pearson) zwischen dem N-Saldo und dem Herbst $\mathrm{N}_{\text {min }}$-Gehalt $(0-90 \mathrm{~cm} \mathrm{I}$ Datenkategorie 1)

\begin{tabular}{|c|c|c|c|}
\hline & Sand & anlehmiger Sand & lehmiger Sand \\
\hline Silo- und Körnermais & $\begin{array}{c}-, 071^{(\text {(n.s.) }} \\
(\mathrm{n}=129)\end{array}$ & $\begin{array}{c}, 018^{(\text {n.s. })} \\
(\mathrm{n}=217) \\
, 026^{(\text {n.s. })} \\
(\mathrm{n}=45)\end{array}$ & $\begin{array}{c}, 289^{\text {(n.s. })} \\
(\mathrm{n}=43)\end{array}$ \\
\hline Silomais & $\begin{array}{c}-, 019^{\text {(n.s.) }} \\
(n=86)\end{array}$ & $\begin{array}{c}, 021^{\text {(n.s.) }} \\
(n=136) \\
-, 058^{(\text {(n.s. })} \\
(n=23)\end{array}$ & $\begin{array}{c}, 321^{\text {(n.s.) }} \\
(\mathrm{n}=27)\end{array}$ \\
\hline Körnermais & $\begin{array}{c}-, 110^{(\text {n.s. })} \\
(\mathrm{n}=43)\end{array}$ & $\begin{array}{c}, 023^{(\text {n.s.s. }} \\
(n=81) \\
, 123^{(\text {(n.s. })} \\
(n=22)\end{array}$ & $\begin{array}{c}107^{(\text {n.s. })} \\
(n=16)\end{array}$ \\
\hline
\end{tabular}

Die Bodenart übte keinen Einfluss auf die Herbst $\mathrm{N}_{\min }$-Menge aus (Abb. 17). So lassen sich mittels des Kuskal-Wallis Tests keine Unterschiede zwischen den verschiedenen Bodenarten und der Herbst $\mathrm{N}_{\min }$-Menge feststellen. 


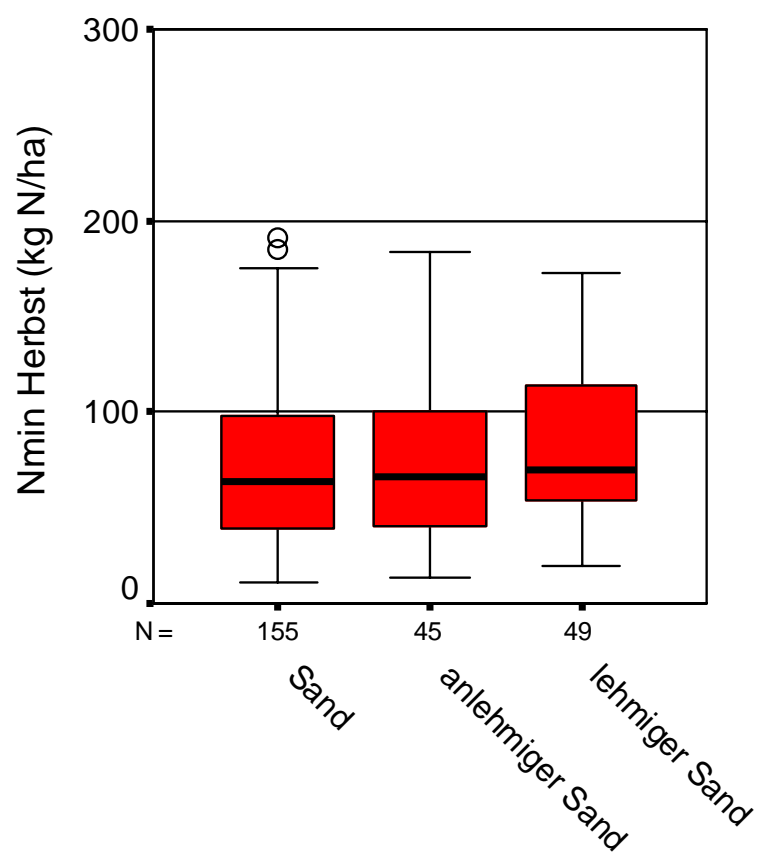

Abb. 17: Herbst $\mathrm{N}_{\min }$ Gehalte ( $\mathrm{kg} \mathrm{N} / \mathrm{ha}$ ) je nach Bodenart (Reichsbodenschätzung I Datenkategorie 1)

In der Abb. 18 wird das Verhältnis von der Herbst $\mathrm{N}_{\min }-$ Menge zum C/N-Verhältnis dargestellt. Es kann keine Beziehung zwischen der herbstlichen $\mathrm{N}_{\min }$-Menge und dem C/N-Verhältnis festgestellt werden. Mittels des Kruskal-Wallis Tests sind auch keine signifikanten Unterschiede zwischen den Datenherkünften nachweisbar.

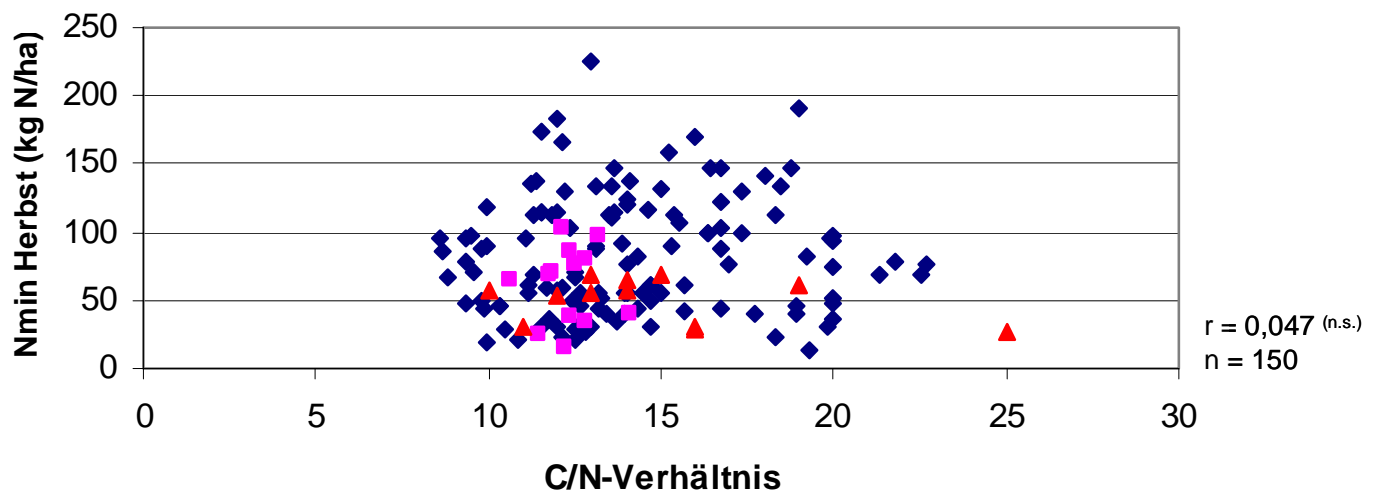

- Weser-Ems $\approx$ Weser-Elbe-Dreieck $\Delta$ Schleswig-Holstein

Abb. 18: Herbst $\mathrm{N}_{\min }$-Gehalte von Silo- und Körnermaisflächen in Abhängigkeit vom $\mathrm{C} / \mathrm{N}$ Verhältnis des Bodens (Korrelation nach Pearson I Datenkategorie 1) 
Einen Einfluss der Viehdichte (< oder $>2 \mathrm{GV} / \mathrm{ha}$ ) auf das $\mathrm{C} / \mathrm{N}$-Verhältnis des Bodens lässt sich per Mann-Whitney Test nicht signifikant belegen. Ob die organische Düngung einen Einfluss auf das $\mathrm{C} / \mathrm{N}-$ Verhältnis hat, wird mittels der Korrelationsanalyse nach Pearson untersucht. Diese kann keine Beziehung zwischen der organischen Düngermenge und dem C/N-Verhältnis finden. Inwieweit das C/N-Verhältnis von der Bodenart abhängig ist, wird für die häufigsten Bodenarten (Sand, anlehmiger Sand, lehmiger Sand), die 92\% aller Flächen umfassten, untersucht. Mit Hilfe des Kruskal-Wallis Tests kann signifikant $(p<0,001)$ festgestellt werden, dass das $\mathrm{C} / \mathrm{N}$-Verhältnis je nach Bodenart unterschiedlich ist. Die Bodenarten mit höheren Tonanteilen weisen ein engeres $\mathrm{C} / \mathrm{N}$-Verhältnis auf.

Der Gesamt-N Gehalt des Bodens (Abb. 19) steht in einem Zusammenhang mit der Herbst $\mathrm{N}_{\min }$-Menge. Die Prüfung der Beziehungen zwischen den Variablen ,Herbst $\mathrm{N}_{\text {min }}$-Gehalt' und ,Gesamt-N' mittels einer Korrelation (Pearson) ergibt einen Korrelationskoeffizienten von 0,229 bei einem Signifikanzniveau von 0,037.

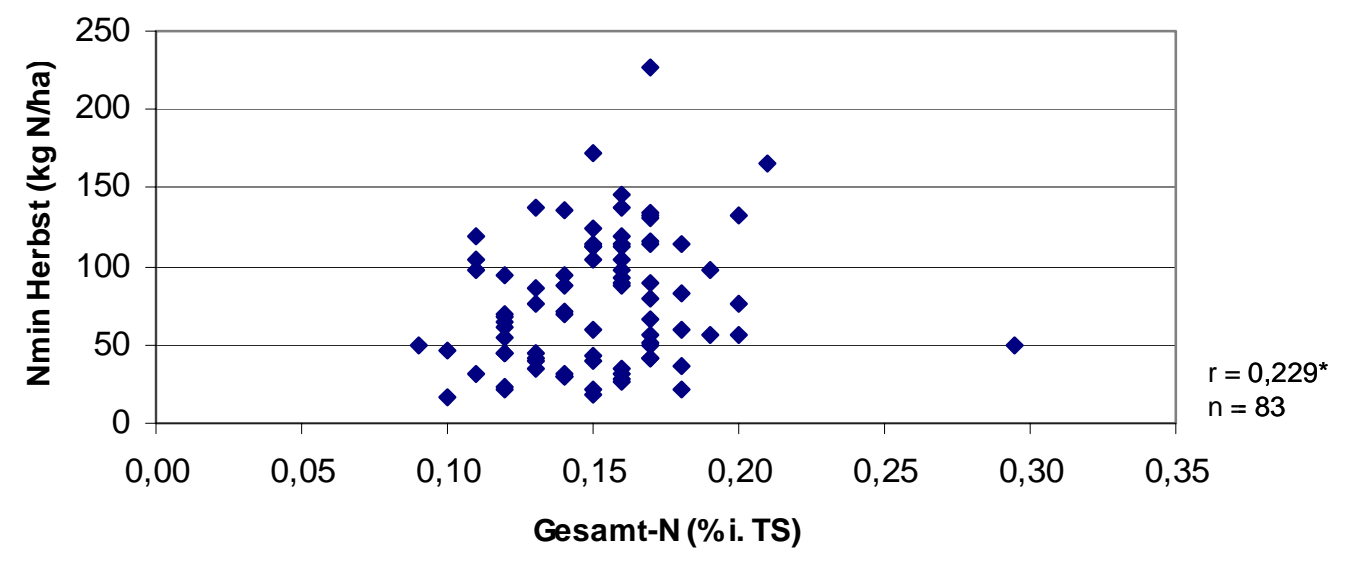

Abb. 19: Herbst $\mathrm{N}_{\min }$-Gehalte von Silo- und Körnermaisflächen in Abhängigkeit vom Gesamt-N Gehalt des Bodens (Korrelation nach Pearson I Datenkategorie 1)

Eine weitere Kategorisierung nach dem Besatz an Großvieheinheiten führt zu keiner weitergehenden Erklärung der Streuung. Der Gesamt-N Gehalt des Bodens zeigt mit einem Korrelationskoeffizienten (Pearson) von 0,288 $(p=0,017)$ eine Beziehung zur ausgebrachten organischen Düngung. Eine Beziehung zwischen dem Viehbesatz und den Gesamt N-Gehalten ist nicht nachzuweisen. Die Untergliederung nach Bodenarten aus der Reichsbodenschätzung führt bei Sandböden $(n=25)$ zu einem höheren Korrelationskoeffizienten (Pearson) von 0,464 ( $p=0,019)$, zwischen den Herbst $\mathrm{N}_{\min }$-Mengen und dem Gesamt- $\mathrm{N}$ Gehalt des Bodens, als bei den anderen 
Bodenarten. Die Gesamt-N Gehalte des Bodens sind zwischen den Bodenarten nach Reichsbodenschätzung nicht signifikant unterschiedlich.

Zwischen dem $\mathrm{C}_{\mathrm{t}}$-Gehalt des Bodens und den Herbst $\mathrm{N}_{\min }$-Mengen besteht ein schwacher Zusammenhang (Abb. 20). Die Korrelation nach Pearson ist mit einem Korrelationskoeffizient (Pearson) von 0,234 signifikant $(p=0,033)$.

Die Streuung nimmt mit steigendem $\mathrm{C}_{\mathrm{t}}$-Gehalt des Bodens zu. Das bedeutet, dass bei höheren Kohlenstoff- bzw. Humusgehalten die Abschätzung des tatsächlichen Herbst $\mathrm{N}_{\min }$-Gehaltes erheblich schwerer fällt. Bei einer Gruppierung nach Standorten aus der Reichsbodenschätzung ist der höchste Korrelationskoeffizient beim Sandboden $(n=25)$ mit $0,453(p=0,023)$ zu finden.

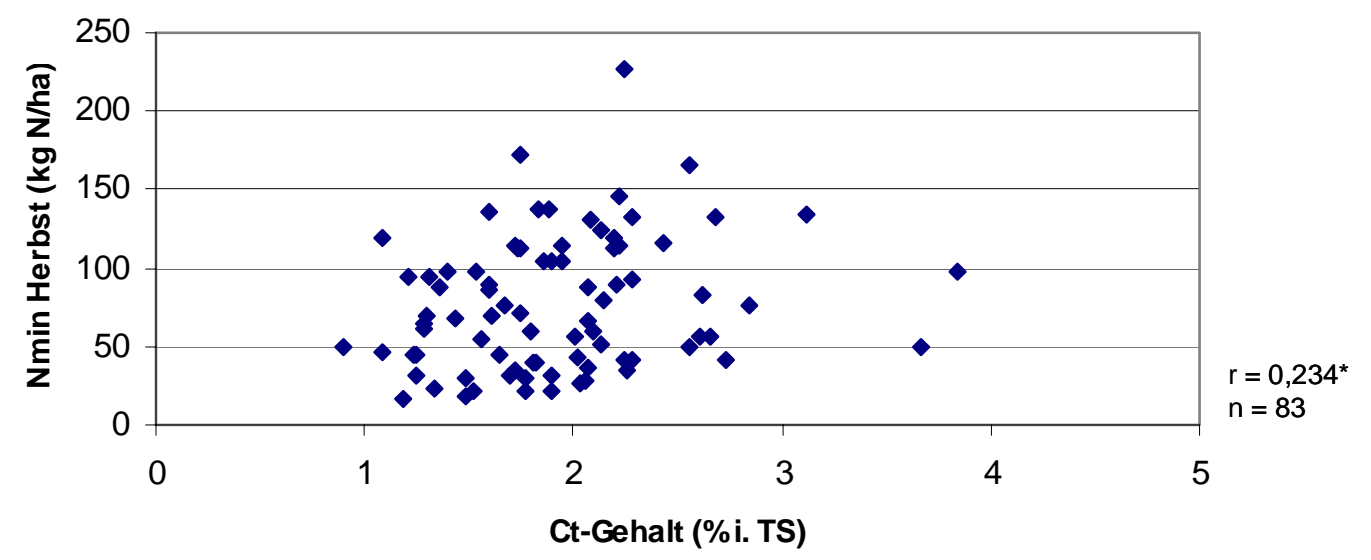

Abb. 20: Herbst $\mathrm{N}_{\min }$-Gehalte von Silo- und Körnermaisflächen in Abhängigkeit vom $\mathrm{C}_{\mathrm{t}}$-Gehalt des Bodens (Korrelation nach Pearson I Datenkategorie 1)

Eine Beziehung zwischen der ausgebrachten organischen Düngermenge und dem $\mathrm{C}_{\mathrm{t}}$-Gehalt kann nicht festgestellt werden. Auch der Viehbesatz zeigt keinen Einfluss auf den $\mathrm{C}_{\mathrm{t}}$-Gehalt des Bodens auf. Die $\mathrm{C}_{\mathrm{t}}$-Gehalte des Bodens variieren zwischen den anderen Bodenarten nach Reichsbodenschätzung nur geringfügig.

Einen praxisbezogenen Auswertungsansatz stellt die Gegenüberstellung der Charakteristika von Flächen dar, die mit ausgesprochen tiefen und ausgesprochen hohen herbstlichen $\mathrm{N}_{\min }$-Gehalten aufwarteten (Tab. 8). Auffallend ist hier, dass die Flächen mit einem niedrigen $\mathrm{N}_{\text {min }}$-Gehalt im Herbst bereits im Frühjahr geringe Mengen mineralisierten Stickstoffs im Boden aufwiesen. Anscheinend sind diese Flächen etwas humusärmer. Im Hinblick auf die Düngung und die Bodenart gibt es keine gerichteten Differenzen zwischen den 25 Flächen mit den höchsten und den geringsten Herbst $\mathrm{N}_{\text {min }}-$ Mengen. 
Tab. 8: Charakteristika von Flächen mit besonders tiefen und auffallend hohen Herbst $\mathbf{N}_{\min }{ }^{-}$ Gehalten (Datenkategorie 1)

\begin{tabular}{|c|c|c|c|}
\hline Variablen & $\begin{array}{l}25 \text { Flächen mit dem } \\
\text { tiefsten Herbst } N_{\text {min }^{-}} \\
\text {Gehalt }\end{array}$ & Mittel & $\begin{array}{l}25 \text { Flächen mit dem } \\
\text { höchsten Herbst } \\
\mathrm{N}_{\min }-\text { Gehalt }\end{array}$ \\
\hline $\begin{array}{l}\text { Herbst } N_{\min } \text {-Gehalt } \\
\text { (kg N/ha) }\end{array}$ & $\begin{array}{c}19 \\
(n=25)\end{array}$ & $\begin{array}{c}76 \\
(n=416)\end{array}$ & $\begin{array}{c}178 \\
(n=25)\end{array}$ \\
\hline $\begin{array}{l}\text { Frühjahrs } N_{\min } \text {-Gehalt } \\
\text { (bis 1. Mai / } \\
\text { kg N/ha)) }\end{array}$ & $\begin{array}{c}32 \\
(n=11)\end{array}$ & $\begin{array}{c}52 \\
(n=167)\end{array}$ & $\begin{array}{c}44 \\
(n=6)\end{array}$ \\
\hline $\begin{array}{l}\text { mineralische Düngung } \\
\text { (kg N/ha) }\end{array}$ & $\begin{array}{c}41 \\
(n=25)\end{array}$ & $\begin{array}{c}50 \\
(n=484)\end{array}$ & $\begin{array}{c}55 \\
(n=24)\end{array}$ \\
\hline $\begin{array}{l}\text { organische Düngung } \\
\text { (kg N/ha) }\end{array}$ & $\begin{array}{c}177 \\
(n=23)\end{array}$ & $\begin{array}{c}162 \\
(n=447)\end{array}$ & $\begin{array}{c}178 \\
(n=22)\end{array}$ \\
\hline Silomaisertrag (dt/ha) & $\begin{array}{c}456 \\
(n=14)\end{array}$ & $\begin{array}{c}444 \\
(n=322)\end{array}$ & $\begin{array}{c}472 \\
(n=18)\end{array}$ \\
\hline Körnermaisertrag (dt/ha) & $\begin{array}{c}89 \\
(n=10)\end{array}$ & $\begin{array}{c}85 \\
(n=138)\end{array}$ & $\begin{array}{c}86 \\
(n=7)\end{array}$ \\
\hline $\begin{array}{l}\text { Bodenart nach } \\
\text { Beraterangaben }\end{array}$ & $\begin{array}{c}11 \times \text { Sand } \\
4 \times \text { anlehmiger Sand } \\
4 x \text { lehmiger Sand }\end{array}$ & $\begin{array}{c}\text { 149x Sand } \\
\text { 50x anlehmiger Sand } \\
60 x \text { lehmiger Sand } \\
\text { 8x stark lehmiger } \\
\text { Sand } \\
\text { 8x sandiger Lehm } \\
\text { 7x Sandmischkultur } \\
\text { 1x Lehm } \\
\text { 1x schwerer Lehm } \\
\text { 2x Moor }\end{array}$ & $\begin{array}{c}6 \times \text { Sand } \\
1 \times \text { anlehmiger Sand } \\
3 \times \text { lehmiger Sand } \\
2 x \text { Sandmischkultur }\end{array}$ \\
\hline C/N-Verhältnis & $\begin{array}{l}13,61 \\
(n=7)\end{array}$ & $\begin{array}{c}14,04 \\
(n=173) \\
\end{array}$ & $\begin{array}{l}14,13 \\
(n=7)\end{array}$ \\
\hline $\mathrm{N}_{\mathrm{t}}$ & $\begin{array}{l}0,14 \\
(n=5)\end{array}$ & $\begin{array}{c}0,15 \\
(\mathrm{n}=90)\end{array}$ & $\begin{array}{l}0,18 \\
(n=3)\end{array}$ \\
\hline $\mathrm{C}_{\mathrm{t}}$ & $\begin{array}{c}1,57 \\
(n=5) \\
\end{array}$ & $\begin{array}{c}1,92 \\
(n=90)\end{array}$ & $\begin{array}{l}2,18 \\
(n=3) \\
\end{array}$ \\
\hline GV-Besatz & $\begin{array}{l}1 x<2 G V / h a \\
5 x>2 G V / h a\end{array}$ & $\begin{array}{c}59 x<2 G V / h a \\
132 x>2 G V / h a\end{array}$ & $\begin{array}{l}4 x<2 G V / h a \\
4 x>2 G V / h a\end{array}$ \\
\hline
\end{tabular}

Inwieweit die Frühjahrs $\mathrm{N}_{\min }$-Gehalte auf die Herbst $\mathrm{N}_{\min }$-Gehalte schließen lassen, zeigt die Tab. 9. In den Jahren 1996 und 1997 besteht ein gesicherter linearer Zusammenhang zwischen den Werten der späten $\mathrm{N}_{\min }$-Beprobungstermine im

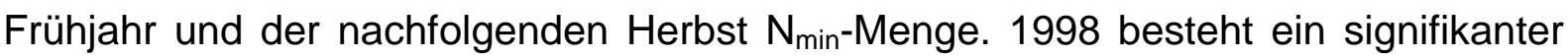
Zusammenhang zwischen der $\mathrm{N}_{\min }$-Menge im Frühjahr bei früher Beprobung und dem Herbst $\mathrm{N}_{\min }$-Gehalt. In diesem Jahr sind nur wenige Flächen spät im Frühjahr beprobt worden, so dass kein signifikanter Zusammenhang zum Herbst $\mathrm{N}_{\min }$-Gehalt festgestellt werden kann.

Bei der Berechnung der Korrelation über alle Erhebungsjahre ergibt sich zwischen der frühen $\mathrm{N}_{\min }$-Beprobung vor Mai und dem Herbst $\mathrm{N}_{\min }$-Gehalt ein Korrelationskoeffizient von $0,200(p=0,028)$ und bei der späten $N_{\text {min }}$-Beprobung ab Mai ein Korrelationskoeffizient von 0,374 $(p=0,006)$. 
Tab. 9: Korrelation (Pearson) zwischen dem Frühjahrs und dem nachfolgenden Herbst $\mathbf{N}_{\min }{ }^{-}$ Gehalt (0-90cm I Datenkategorie 1)

\begin{tabular}{|c|c|c|c|c|}
\hline $\begin{array}{l}\text { Probenahme } \\
\text { im Frühiahr }\end{array}$ & 1996 & 1997 & 1998 & 1999 \\
\hline vor 1. Mai & $\begin{array}{l}133^{(\text {n.S.) }} \\
(n=21)\end{array}$ & $\begin{array}{c}183^{(\text {n.S. })} \\
(n=42)\end{array}$ & $\begin{array}{l}, 402^{*} \\
(n=26)\end{array}$ & $\begin{array}{l}-, 022^{(\text {n.S. })} \\
(n=27)\end{array}$ \\
\hline ab 1. Mai & $\begin{array}{l}, 653^{*} \\
(n=13)\end{array}$ & $\begin{array}{l}, 493^{\star *} \\
(n=28)\end{array}$ &, $466^{(\text {n.s. })}$ & $\begin{array}{c}2222^{(\text {n.s. })} \\
(\mathrm{n}=9)\end{array}$ \\
\hline
\end{tabular}

Eine schrittweise multiple lineare Regression mit dem Herbst $\mathrm{N}_{\min }$-Gehalt als abhängige Variable und den bisher in Streudiagrammen dargestellten unabhängigen Variablen kann auf Grund der nicht erfüllten Eingangsvoraussetzungen nicht durchgeführt werden.

\section{Einfluss der Vor- und Zwischenfrüchte auf die Frühjahrs und Herbst $\mathbf{N}_{\text {min}^{-}}$ Menge}

Zur Aufdeckung der Wirkung des Zwischenfruchtbaus vor dem Maisanbau werden die zur Verfügung stehenden Daten in zwei Fallgruppen geteilt, mit einer Bodenbedeckung (Zwischenfruchtanbau) oder ohne eine Bodenbedeckung (Brache). Ein sich anschließender Vergleich zwischen Brache und Bodenbedeckung mittels Mann-Whitney Test zeigt keine Unterschiede in den zugehörigen Frühjahrs oder darauf folgenden Herbst $\mathrm{N}_{\min }$-Gehalten. In Abb. 21 werden die $\mathrm{N}_{\min }$-Gehalte vom Frühjahr und Herbst mittels Boxplot von den mit Zwischenfrüchten bestellten Flächen dargestellt, welche auf mindestens 2 Prozent der erhobenen Flächen standen. Da nicht von allen Flächen, auf denen Zwischenfrüchte angebaut worden sind, Bodenproben gezogen wurden, ist die Anzahl der in der Abb. 21 dargestellten Werte je Zwischenfrucht Z. T. niedrig.

In der Abb. 21 weist die Brache, der Winterroggen, der Winterraps und das Feldgras im Frühjahr die geringsten $\mathrm{N}_{\text {min }}$-Gehalte, der Ölrettich hingegen die höchsten Werte auf. Der Einfluss der Zwischenfrüchte auf die Frühjahrs $\mathrm{N}_{\min }$-Mengen ist signifikant unterschiedlich (Kruskal-Wallis Test; $p=0,013$ ).

Im Herbst sind kaum Unterschiede zwischen den Zwischenfrüchten und den Herbst $\mathrm{N}_{\text {min }}$-Gehalt nach dem Anbau von Mais auszumachen. Lediglich die Flächen mit Winterraps weisen einen geringfügig höheren Herbst $\mathrm{N}_{\min }$-Gehalt auf. Mit dem Kruskall-Wallis Test kann kein Unterschied in den Herbst $\mathrm{N}_{\text {min }}$-Mengen zwischen den verschiedenen Zwischenfrüchten nachgewiesen werden. Die ausgebrachte Düngermenge zur Hauptfrucht Mais ist je nach der Zwischenfrucht bzw. Brache signifikant unterschiedlich (Kruskal-Wallis Test / p $<0,001$ ). Die geringsten 
Düngermengen werden nach der Brache mit ungefähr 162kg N/ha im Mittel appliziert. Die höchsten Düngermengen werden nach Gras und Ölrettich ausgebracht. Die mittlere Düngermenge liegt bei Gras mit $247 \mathrm{~kg} \mathrm{~N} / \mathrm{ha}$ unter der von Ölrettich mit $280 \mathrm{~kg} \mathrm{~N} / \mathrm{ha}$. Die weiteren ausgebrachten Düngermengen liegen im Mittel zwischen $186 \mathrm{~kg} \mathrm{~N} / \mathrm{ha}$ (Phacelia) und $209 \mathrm{~kg} \mathrm{~N} / \mathrm{ha}$ (Winterroggen). Die Maisflächen nach Senf erhielten eine mittlere Düngermenge von $190 \mathrm{~kg} \mathrm{~N} / \mathrm{ha}$ und nach Winterraps von $207 \mathrm{~kg} \mathrm{~N} / \mathrm{ha}$.
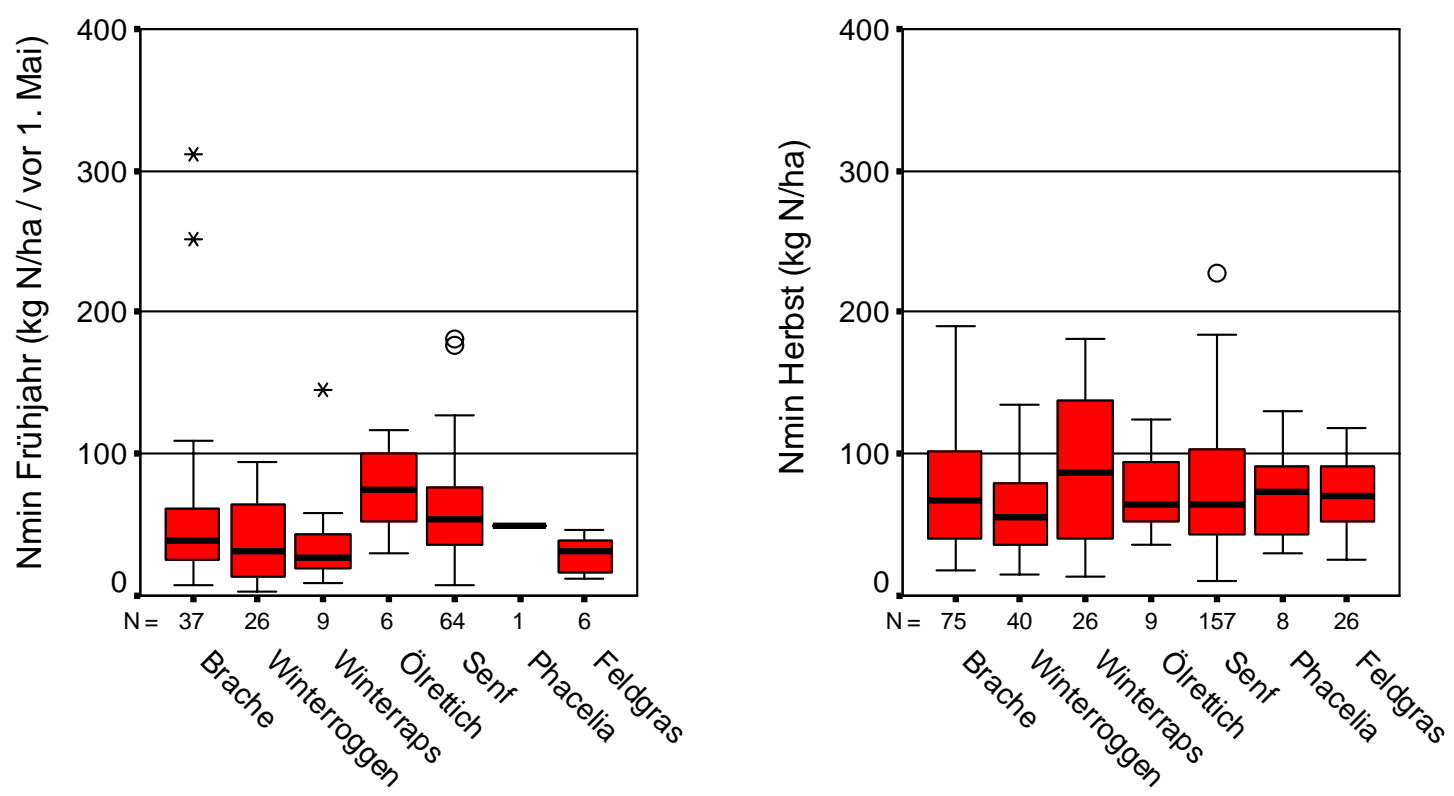

Abb. 21: Verteilung der Frühjahrs $\mathbf{N}_{\min }$-Gehalte nach dem Anbau von Zwischenfrüchten zur Hauptfrucht Mais (links/Probenahmetermin vor Mai), sowie Verteilung der Herbst $\mathbf{N}_{\min }$-Gehalte nach erfolgtem Maisanbau (rechts), jeweils in $\mathrm{kg} \mathrm{N} / \mathrm{ha}$ in $0-90 \mathrm{~cm}$ Bodentiefe (Datenkategorie 1)

Der Einfluss der Vorfrucht auf den Frühjahrs und Herbst $\mathrm{N}_{\mathrm{min}}$-Gehalt wird in der Abb. 22 dargestellt. Im Fall von Getreide wird nicht danach differenziert, ob das Stroh auf dem Feld verblieben oder abgefahren worden war. Ein Effekt des Strohs kann nicht festgestellt werden. Die Vorfrucht hat weder auf die $\mathrm{N}_{\min }$-Mengen im Frühjahr noch im Herbst einen signifikanten Einfluss. Es gibt bei den $\mathrm{N}_{\text {min }}$-Vorräten sowohl im Frühjahr wie im Herbst keinen Hinweis darauf, dass der Mais in der Selbstfolge größere Rest $\mathrm{N}_{\text {min }}$-Mengen hinterlässt als bei Fruchtwechsel. 

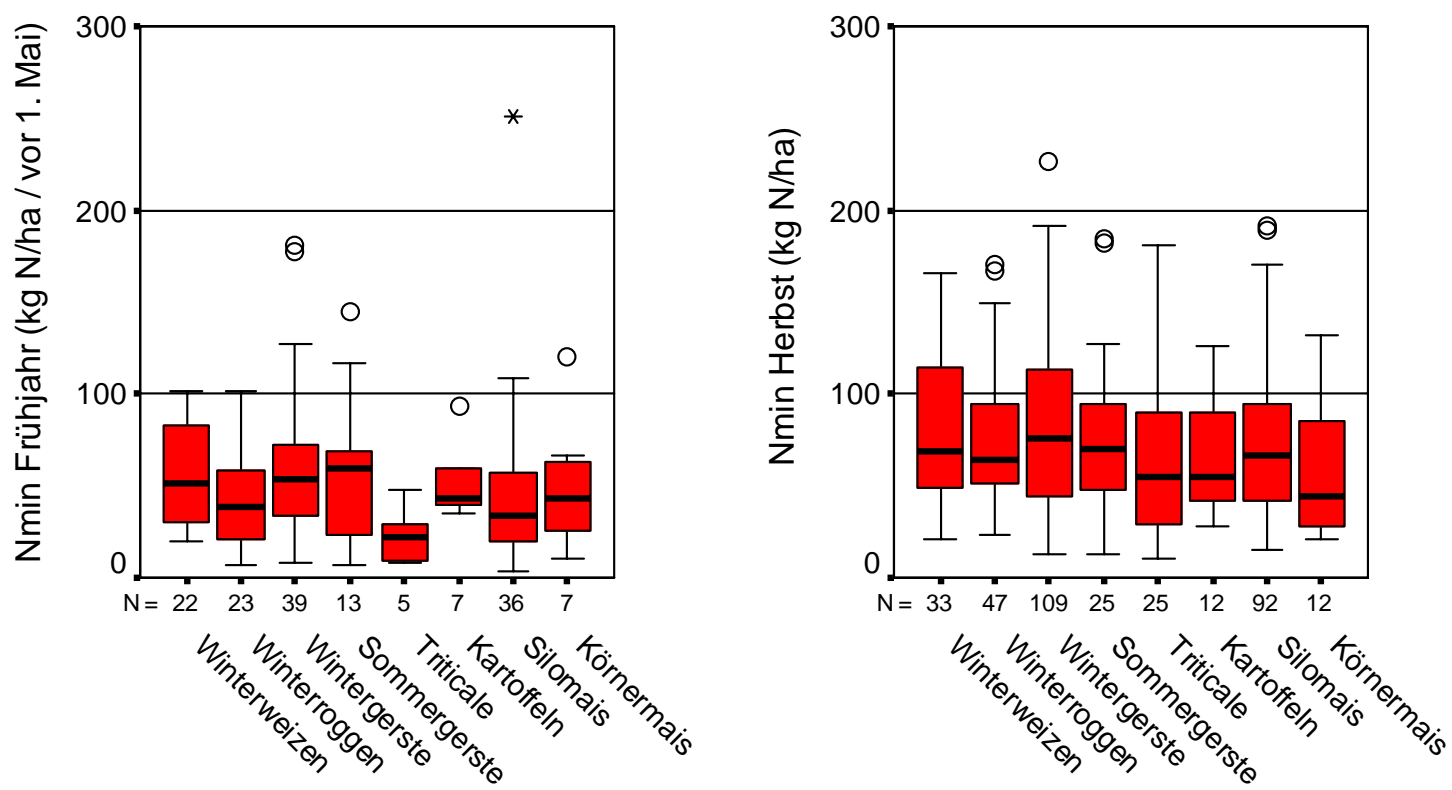

Abb. 22: Verteilung der Frühjahrs $\mathrm{N}_{\min }$-Gehalte zur Hauptfrucht Mais (links/Probenahmetermin vor Mai) sowie Verteilung der Herbst $\mathbf{N}_{\min }$-Gehalte nach erfolgtem Maisanbau (rechts), jeweils in $\mathrm{kg} \mathrm{N} / \mathrm{ha}$ in $0-90 \mathrm{~cm}$ Bodentiefe, in Abhängigkeit von der Art der Vorfrucht (Datenkategorie 1)

\section{Einfluss der Witterung auf die Herbst $\mathrm{N}_{\text {min }}$-Menge}

Welchen Einfluss die Witterung auf die Ausprägung der Herbst $\mathrm{N}_{\text {min }}-$ Mengen hat, wird anhand der Tab. 10 dargestellt. Die Witterungsverläufe in den Untersuchungsjahren auf den verschiedenen Standorten sind im Anhang 7 und Anhang 8 zusammengestellt. Die Temperaturen schwanken über die Jahre und Standorte von $8^{\circ} \mathrm{C}$ im Mittel des Jahres 1996 bis zu 10,5 C im Jahr 1999. Im Jahr 1996 war das Frühjahr relativ kalt. Das Jahr 1996 war mit 614 mm das niederschlagsärmste. In den Jahren 1997 und 1999 fielen 738 bzw. 819 mm Niederschlag. Das Jahr 1998 war dagegen extrem niederschlagsreich. So fielen über das Jahr im Mittel über 1099 mm. Im Diagramm (Anhang 8) der Niederschläge fällt im Jahr 1998 besonders der Juni und Oktober auf. Die Niederschläge in diesen Monaten waren überdurchschnittlich ergiebig.

Inwieweit die Temperaturen und die Niederschläge einzelner Monate vor der Beprobung Auswirkungen auf die Herbst $\mathrm{N}_{\min }$-Gehalte hatten, wird in Tab. 10 dargestellt. Die Temperatur weist im Mai und September schwache negative und im Juli und August schwache positive Korrelationskoeffizienten auf. Die Korrelationskoeffizienten der Niederschlagsmonate vor der Herbst $\mathrm{N}_{\min }$-Beprobung sind generell negativ. Das bedeutet, dass mit zunehmenden Niederschlägen die vorgefundene $\mathrm{N}_{\min }$-Menge im Herbst niedriger ist. Dies wird durch den negativen 
Korrelationskoeffizienten $(r=-0,217)$ mit einem Signifikanzniveau von $<0,001$ bestätigt, der sich zwischen dem Herbst $\mathrm{N}_{\min }$-Gehalt und der Summe der Niederschläge von Mai bis einschließlich September ergibt.

Tab. 10: Korrelation (Kendall) zwischen der Witterung (Temperatur und Niederschlag) und dem Herbst $\mathrm{N}_{\text {min }}$-Gehalt (0-90cm / Datenkategorie 1)

\begin{tabular}{lcccccc}
\hline & & \multicolumn{5}{c}{ Monat vor der Beprobung } \\
& Mai & Juni & Juli & August & September & Oktober \\
\hline Temperatur &,$- 267^{* *}$ &, $023^{(n . s .)}$ &, $159^{* *}$ &, $262^{* *}$ &,$- 129 * *$ &, $021^{(n . s .)}$ \\
& $(n=362)$ & $(n=362)$ & $(n=362)$ & $(n=362)$ & $(n=337)$ & $(n=214)$ \\
Niederschlag &,$- 225^{* *}$ &,$- 257^{* *}$ &,$- 132^{* *}$ &,$- 001^{(n . s .)}$ &,$- 221^{* *}$ &,$- 226^{* *}$ \\
& $(n=362)$ & $(n=362)$ & $(n=357)$ & $(n=359)$ & $(n=337)$ & $(n=214)$ \\
\hline
\end{tabular}

Wie hoch die potentielle Verdunstung (nach HAUDE) in den einzelnen Jahren war, ist dem Anhang $9 \mathrm{zu}$ entnehmen. Es zeigt sich, dass das Jahr 1998 besonders im Hochsommer eine geringere Evapotranspiration aufwies als die anderen drei Haupterhebungsjahre. Im Juli 1999 sorgten die höheren Temperaturen für eine stärkere Evapotranspiration. 


\subsection{Auswertung der Daten aus Erhebungen (Datenkategorie 2)}

\subsubsection{Variabilität der untersuchten Merkmale}

Bei der Auswertung der Daten aus bundesweiten Erhebungen werden zunächst die verschiedenen Merkmale und deren Variabilität mittels der beschreibenden Statistik dargestellt. Je nach Merkmal stehen zwischen 62 und 408 Werte zur Verfügung. Zunächst werden einige Häufigkeitsverteilungen von den wesentlichen Prüfmerkmalen der 414 Flächen dargestellt. Die Beprobung der Herbst $\mathrm{N}_{\min }$-Gehalte $(0-90 \mathrm{~cm})$ fand nach der Ernte im Zeitraum der 39. Kalenderwoche und bei Vegetationsende in der 47. Kalenderwoche statt. Die Mittelwerte der beiden $\mathrm{N}_{\text {min- }}$ Beprobungstermine liegen mit 75 und $74 \mathrm{~kg} \mathrm{~N} \mathrm{~min}_{\text {/ha }}$ nahe beieinander (Abb. 23). Da in dem Beprobungszeitraum von 1985 bis in das Jahr 2000 die Anzahl der zur Verfügung stehenden Flächen je Jahr stark unterschiedlich waren, werden die Jahreseffekte auf den Herbst $\mathrm{N}_{\min }$-Gehalt nicht untersucht.
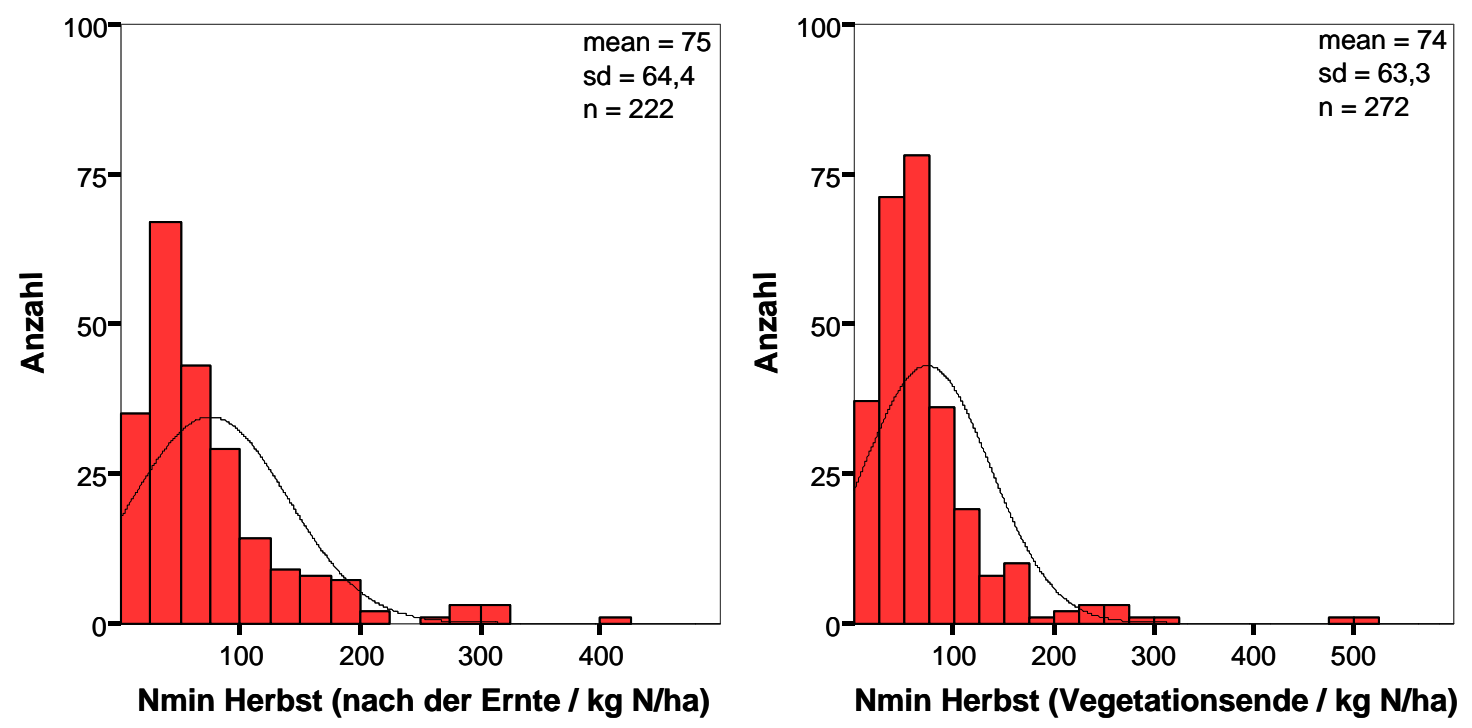

Abb. 23: Häufigkeitsverteilung der Herbst $\mathrm{N}_{\min }-$ Gehalte $(0-90 \mathrm{~cm})$ nach der Ernte und am Vegetationsende (Datenkategorie 2)

Die Frühjahrs $\mathrm{N}_{\text {min }}$-Gehalte $(0-90 \mathrm{~cm})$ wurden im Zeitraum der 10. Kalenderwoche von 312 Flächen ermittelt. Diese weisen im Mittel eine Frühjahrs $N_{\min }-$ Menge von $53 \mathrm{~kg}$ $\mathrm{N}_{\text {min }} /$ ha auf. Die Häufigkeitsverteilung wird in Abb. 24 dargestellt. 


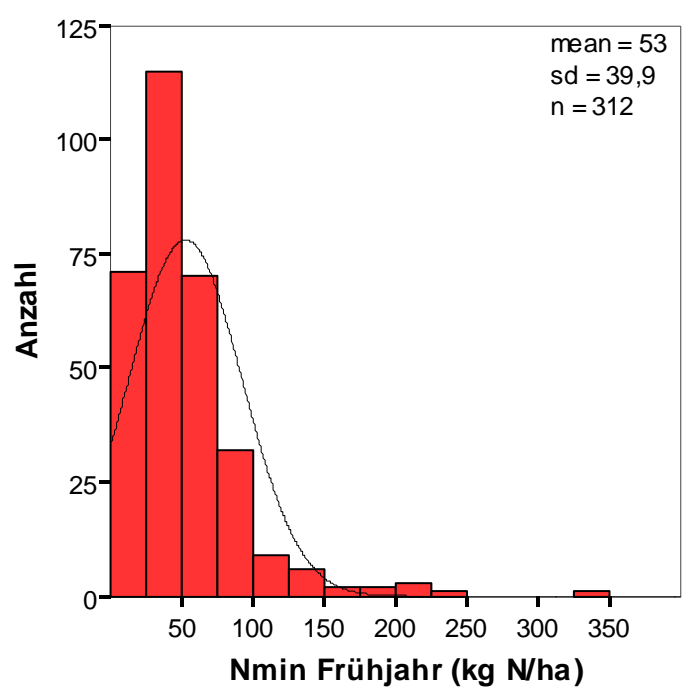

Abb. 24: Häufigkeitsverteilung der Frühjahrs $\mathrm{N}_{\min }$-Gehalte (0-90cm / Datenkategorie 2)

Die ausgebrachte Düngermenge (mineralisch und organisch) liegt von 392 Flächen vor. Im Mittel wurde $143 \mathrm{~kg} \mathrm{~N} / \mathrm{ha}$ gedüngt (Abb. 25).

Die organische Düngung der Flächen gliedert sich anteilig in Gülle $(73,5 \%)$, Festmist $(21,4 \%)$, Jauche $(1,5 \%)$, Klärschlamm (1\%) und Sonstige (2,6\%). Eine separate Betrachtung der mineralischen und organischen Düngung zeigt, dass die Flächen im Mittel mit $89 \mathrm{~kg} \mathrm{~N} / \mathrm{ha}$ mineralisch und $114 \mathrm{~kg} \mathrm{~N} / \mathrm{ha}$ organisch gedüngt wurden. Die mineralische und organische Düngermenge unterscheidet sich zwischen den Institutionen, die die Daten zur Verfügung stellen. Im Nmon-Projekt wurden im Mittel $149 \mathrm{~kg} \mathrm{~N} / \mathrm{ha}$, auf den Vergleichsflächen im Mittel $109 \mathrm{~kg} \mathrm{~N} / \mathrm{ha}$ und bei den Nitratraster-Daten $212 \mathrm{~kg} \mathrm{~N} / \mathrm{ha}$ ausgebracht (Anhang 10). 


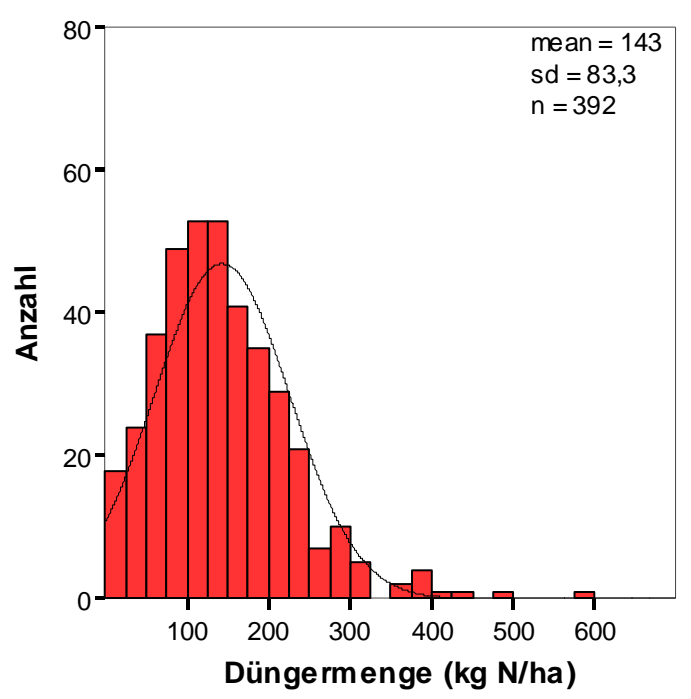

Abb. 25: Häufigkeitsverteilung der aufgewendeten mineralischen und organischen Düngermenge in $\mathrm{kg} \mathrm{N} / \mathrm{ha}$ (Datenkategorie 2)

Die Silo- und Körnermaiserträge wurden durch die Institutionen präzise erhoben. Es liegen von 219 Flächen die Silomaiserträge vor (Anhang 11). Im Mittel wurden 478 $\mathrm{dt} / \mathrm{ha}$ Frischmasse bzw. $154 \mathrm{dt} / \mathrm{ha}$ Trockenmasse geerntet. Des Weiteren sind von 72 Flächen die Körnermaiserträge bekannt (Anhang 12). Hier wurden im Mittel $113 \mathrm{dt} / \mathrm{ha}$ Frischmasse bzw. $77 \mathrm{dt} / \mathrm{ha}$ Trockenmasse geerntet. Die berechneten N-Salden von 286 Flächen lagen im Mittel bei -30 kg N/ha (Abb. 26).

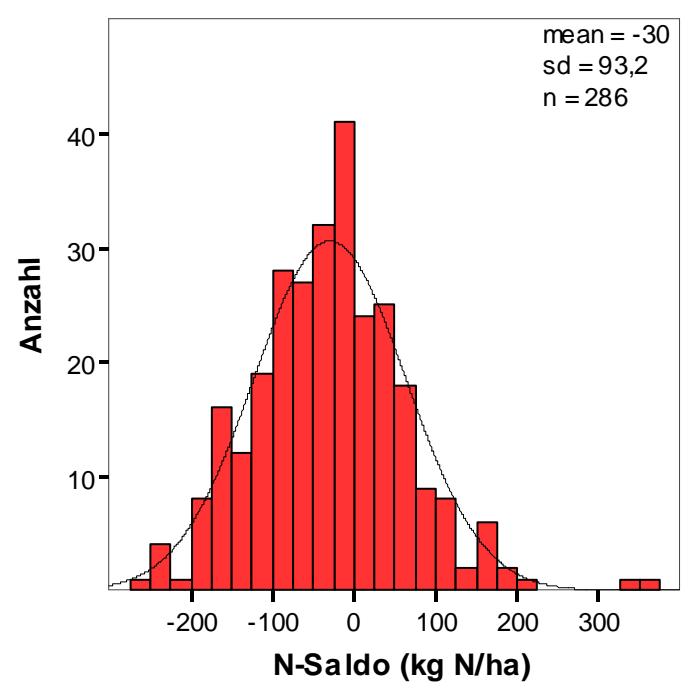

Abb. 26: Häufigkeitsverteilung der N-Salden (kg N/ha / Datenkategorie 2) 
Die Vorfrüchte der Maisflächen sind von 289 Silomais- und 98 Körnermaisflächen bekannt (Anhang 13). Von 9 weiteren Flächen (vergleiche Tab. 2) ist die exakte Nutzungsart (Silomais oder Körnermais) des Maises nicht bekannt. Auf 69\% der 387 Flächen von denen die Vorfrucht bekannt ist, standen Winterweizen (99 Flächen), Wintergerste (69 Flächen), Sommergerste (51 Flächen) und Silomais (49 Flächen) als Vorfrucht vor dem Maisanbau.

Die Art der Zwischenfrucht vor dem Maisanbau wird in dieser Datenkategorie danach unterschieden, ob es eine Brache, keine Leguminose oder eine Leguminose ist. Von 253 Silomais- und 82 Körnermaisflächen liegen Angaben hierzu vor (Anhang 14). Von 15 weiteren Flächen (vergleiche Tab. 2) ist die Nutzungsart des Maises nicht bekannt oder aber ein Gemenge aus undefinierten Früchten wurde angebaut. 59\% der 335 Flächen lagen vor dem Anbau von Mais brach. Lediglich auf einer Fläche stand eine Leguminose.

\section{Daten zu der Betriebsstruktur}

In dieser Datenkategorie stehen nur einige Angaben zum Viehbesatz als Betriebsstruktur beschreibendes Kriterium zur Verfügung. Dieses ist darin begründet, dass die Untersuchungsansätze im Schwerpunkt bei der Düngung und Nutzung der bewirtschafteten Flächen liegen. Zu 62 Flächen können Angaben zum Viehbesatz des bewirtschaftenden Betriebes zugeordnet werden. Der mittlere Viehbesatz lag bei 1,41 GV/ha (Abb. 27).

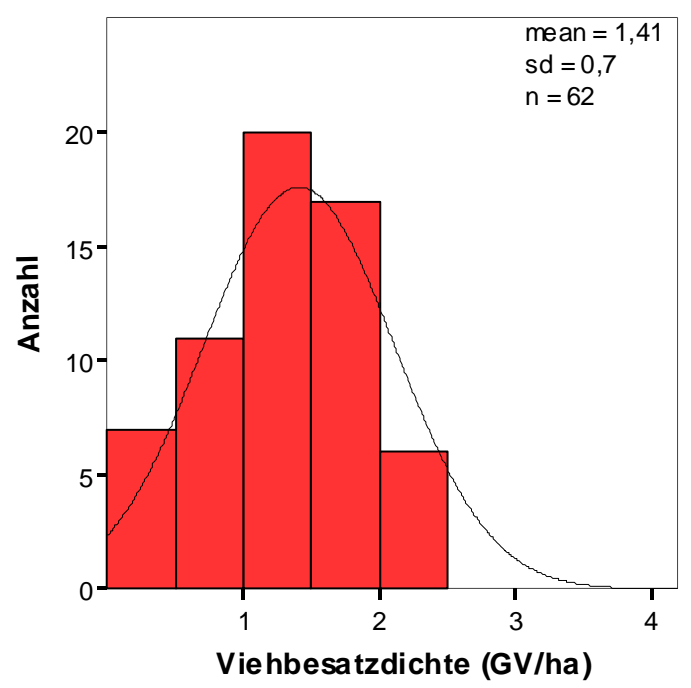

Abb. 27: Häufigkeitsverteilung der Viehbesatzdichten (GV/ha / Datenkategorie 2) 


\section{Standortbezogene Daten und deren Struktur}

Die Bodenarten werden in dieser Kategorie nach einem festen Schema gruppiert (Anhang 6). Insgesamt liegen von 386 Silo- und Körnermaisflächen Angaben zu der Bodenartgruppe vor. Von 22 weiteren Flächen (Tab. 2) liegt entweder die Nutzungsart des Maises nicht vor oder es handelt sich um eine nur schwer in dieses Schema einzusortierende Bodenart (Moor $=6$ Flächen). Auf 210 Flächen liegt der Tonanteil zwischen 12 und 25\%. 101 Flächen weisen einen Tonanteil von mehr als $25 \%$ und 75 Flächen von kleiner $12 \%$ auf (Abb. 28).

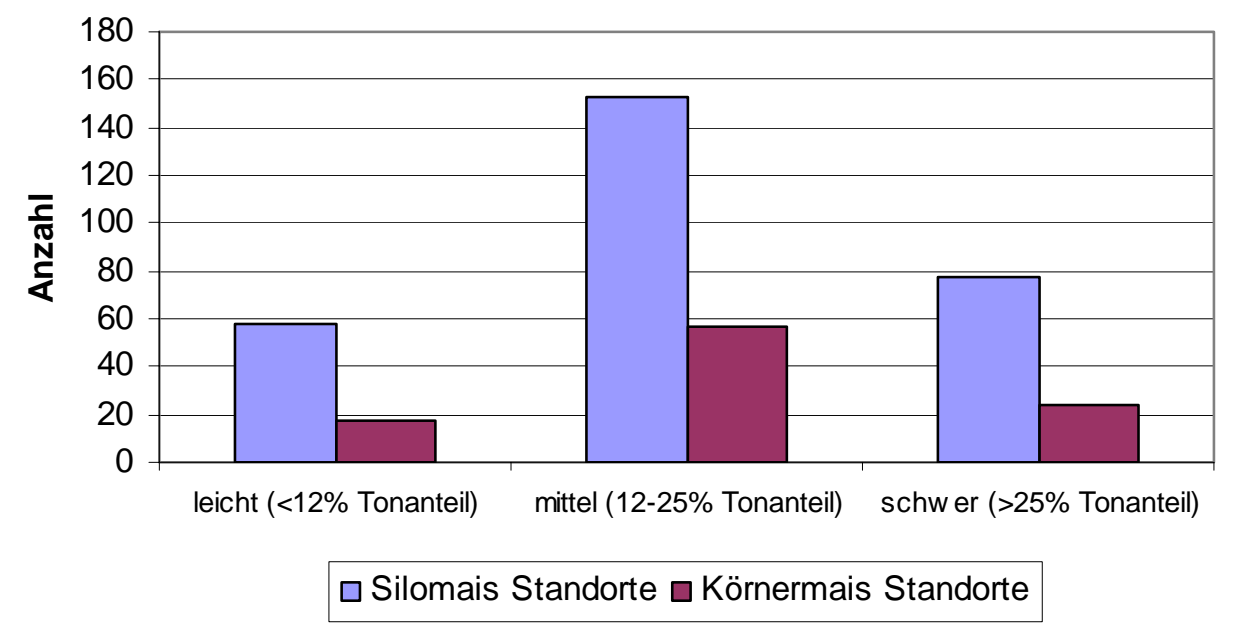

Abb. 28: Häufigkeitsverteilung der Silo- und Körnermaisflächen nach gruppierten Bodenarten (Datenkategorie 2)

Auf 196 Flächen wurde das C/N-Verhältnis des Bodens in der Ackerkrume erhoben. Das Mittel des C/N-Verhältnisses liegt bei 9,8 (Abb. 29). Die aus dem $N_{m o n}$-Projekt eingeflossen $\mathrm{C} / \mathrm{N}-$ Verhältnisse liegen im Mittel bei 8,3 und die aus den NitratrasterDaten bei 16,4 . 


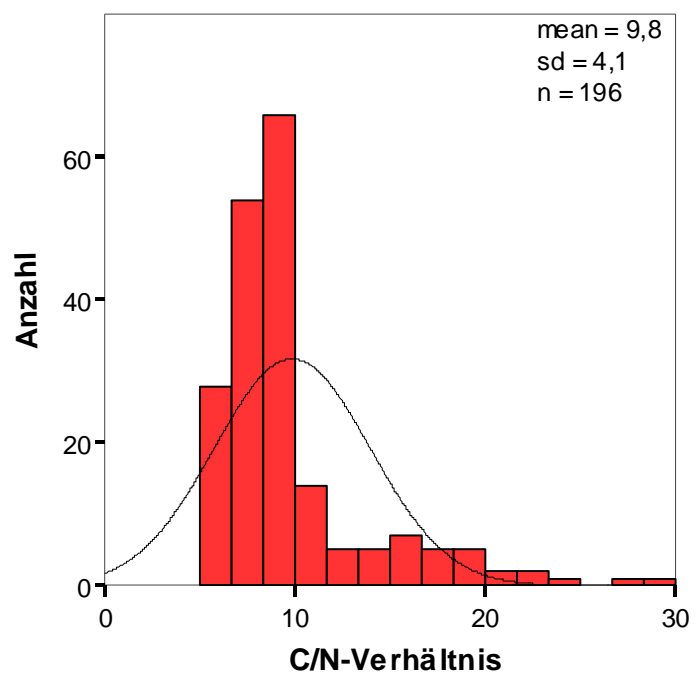

Abb. 29: Häufigkeitsverteilung der C/N-Verhältnisse (über alle Bodenarten / Datenkategorie 2)

Als weiteres Boden beschreibendes Merkmal liegt der pH-Wert von 314 Flächen vor. Dieser beträgt im Mittel 6,6 (sd =0,7). Der $\mathrm{P}_{2} \mathrm{O}_{5}$ und $\mathrm{K}_{2} \mathrm{O}-$ Gehalt ist ebenfalls von 314 Flächen bekannt und liegt im Mittel bei $21 \mathrm{mg} \mathrm{P}_{2} \mathrm{O}_{5}(\mathrm{sd}=11,4)$ und $24 \mathrm{mg} \mathrm{K}_{2} \mathrm{O}$ $(\mathrm{sd}=11)$ je $100 \mathrm{~g}$ Boden.

\subsubsection{Analytische Statistik}

In der analytischen Statistik wird zunächst die Faktorenanalyse, die Aufschluss über die Struktur der Daten geben soll, eingesetzt. Die in die Faktorenanalyse eingeflossenen Variablen wurden anhand des KMO-Maßes und des MSA-Wertes ausgewählt. Aus der Tab. 11 geht hervor, dass die ersten beiden Komponenten (Faktoren) 68\% der Varianz beschreiben.

Tab. 11: Eigenwerte und erklärte Varianzen der Faktorenanalyse (Datenkategorie 2)

\begin{tabular}{lcc}
\hline & Komponente 1 & Komponente 2 \\
\hline Eigenwert & 2,18 & 1,91 \\
\% der Varianz & 36,36 & 31,77 \\
Kumulierte \% & 36,36 & 68,13 \\
\hline
\end{tabular}

Aus welchen Variablen sich die Komponenten zusammensetzen zeigt die rotierte Komponentenmatrix (Tab. 12). Die dargestellte Faktorladung entspricht der Korrelation zwischen einer Variablen und einem Faktor. Je höher diese Ladung ist, desto besser misst diese Variable (Item) den Faktor (relevante = fett gedruckt). Die 
mineralische und organische Düngermenge, der N-Saldo und die Ackerzahl weisen in der Komponente 1 die höchsten Faktorladungen auf. In der Komponente 2 sind dieses der Frühjahrs $\mathrm{N}_{\min }$-Gehalt, der Gesamt-N Gehalt des Bodens und die organische Düngung.

Tab. 12: Rotierte Komponentenmatrix mit der entsprechenden Faktorladung (Datenkategorie 2)

\begin{tabular}{|c|c|c|}
\hline \multirow[t]{2}{*}{ Variablen } & \multicolumn{2}{|c|}{ Komponente } \\
\hline & 1 & 2 \\
\hline $\mathrm{N}_{\min }$ Frühjahr (0-90cm) & 0,006 & 0,878 \\
\hline Gesamt-N (Boden) & 0,140 & 0,647 \\
\hline Düngung (min.+org.) & 0,768 & 0,458 \\
\hline Düngung (org.) & 0,553 & 0,675 \\
\hline N-Saldo & 0,848 & 0,219 \\
\hline Ackerzahl & $-0,741$ & 0,060 \\
\hline
\end{tabular}

Aus diesen ermittelten Komponenten sind mittels Regression Faktorwerte geschätzt worden. Diese dienen als Variablen in der weiteren Untersuchung. Der Einfluss der Faktorwerte auf den Herbst $\mathrm{N}_{\min }$-Gehalt wurde dann mit der partiellen Korrelation untersucht. Der partielle Korrelationskoeffizient zwischen Komponente 1 und dem Herbst $\mathrm{N}_{\min }$-Gehalt, kontrolliert von Komponente 2, ist 0,102 und nicht signifikant. Zwischen Komponente 2 und dem Herbst $\mathrm{N}_{\min }$-Gehalt $(r=0,534)$ besteht unter Kontrolle von Komponente 1 ein signifikanter Zusammenhang $(p<0,001)$. 


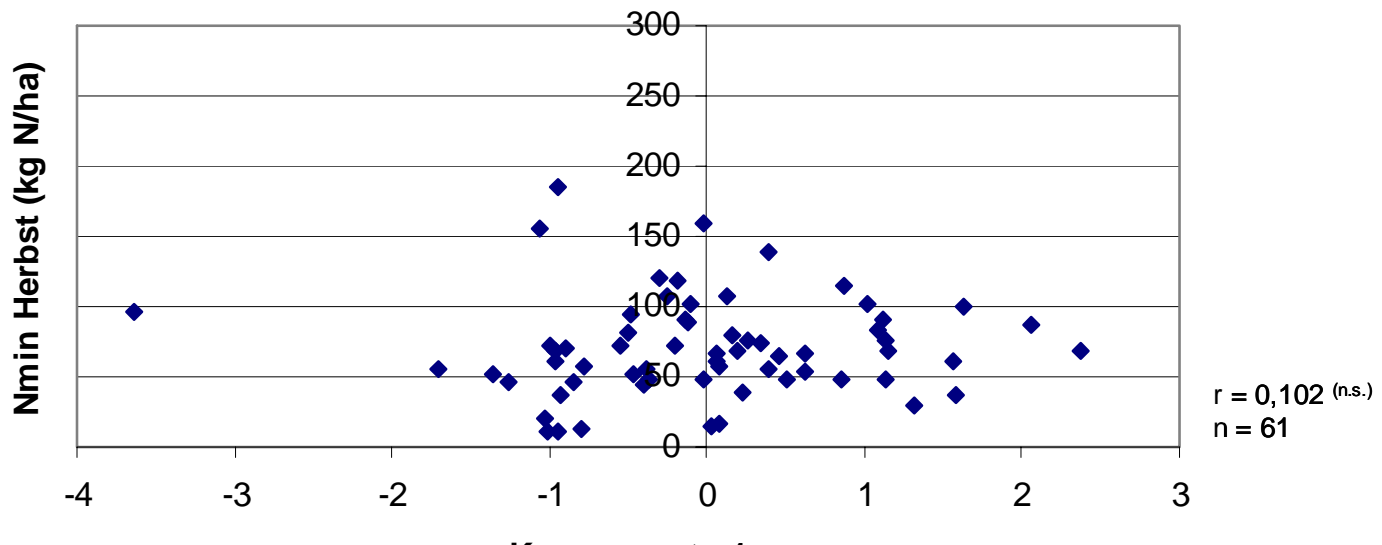

Komponente 1

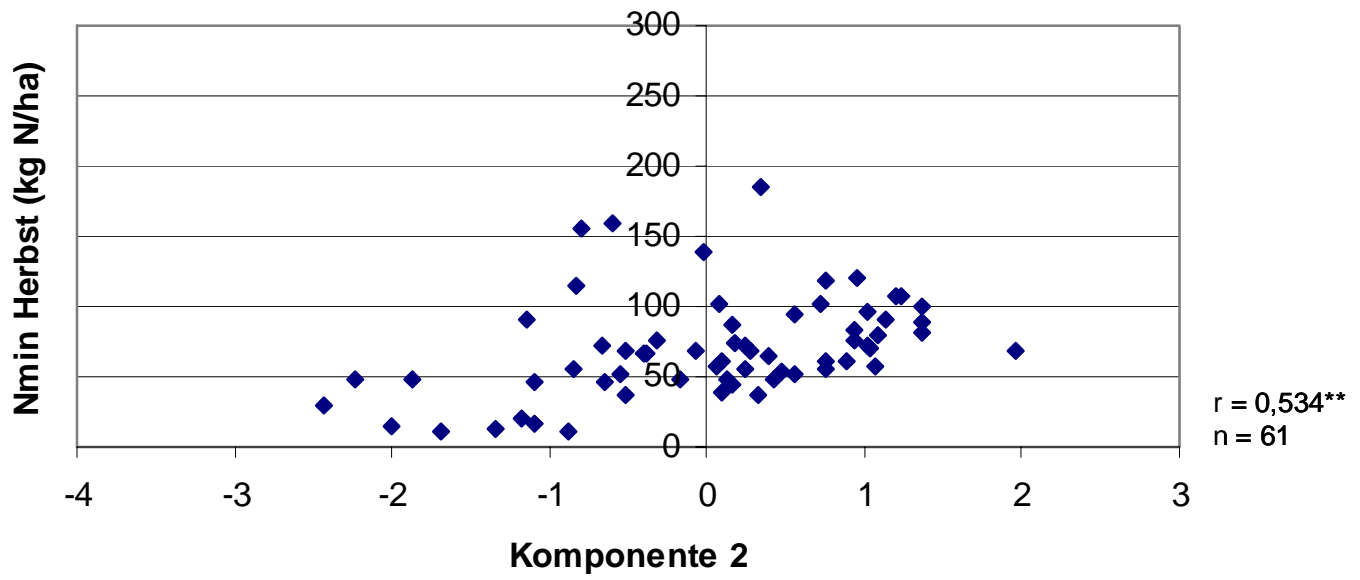

Abb. 30: Beziehung zwischen dem Herbst $\mathrm{N}_{\min }$-Gehalt (Vegetationsende) und den einzelnen Komponenten der Faktorenanalyse (partielle Korrelation I Datenkategorie 2)

Nach der Analyse der Struktur der Daten mittels der Faktorenanalyse werden die Daten in den sich anschließenden Diagrammen ausgewählten Variablen gegenübergestellt.

In Abb. 31 wird das Verhältnis zwischen Herbst $\mathrm{N}_{\min }$-Gehalt zum Vegetationsende und der ausgebrachten mineralischen und organischen Düngermenge von 258 Flächen dargestellt. Zwischen der Düngermenge und dem Herbst $\mathrm{N}_{\min }$-Gehalt besteht keine Beziehung. 


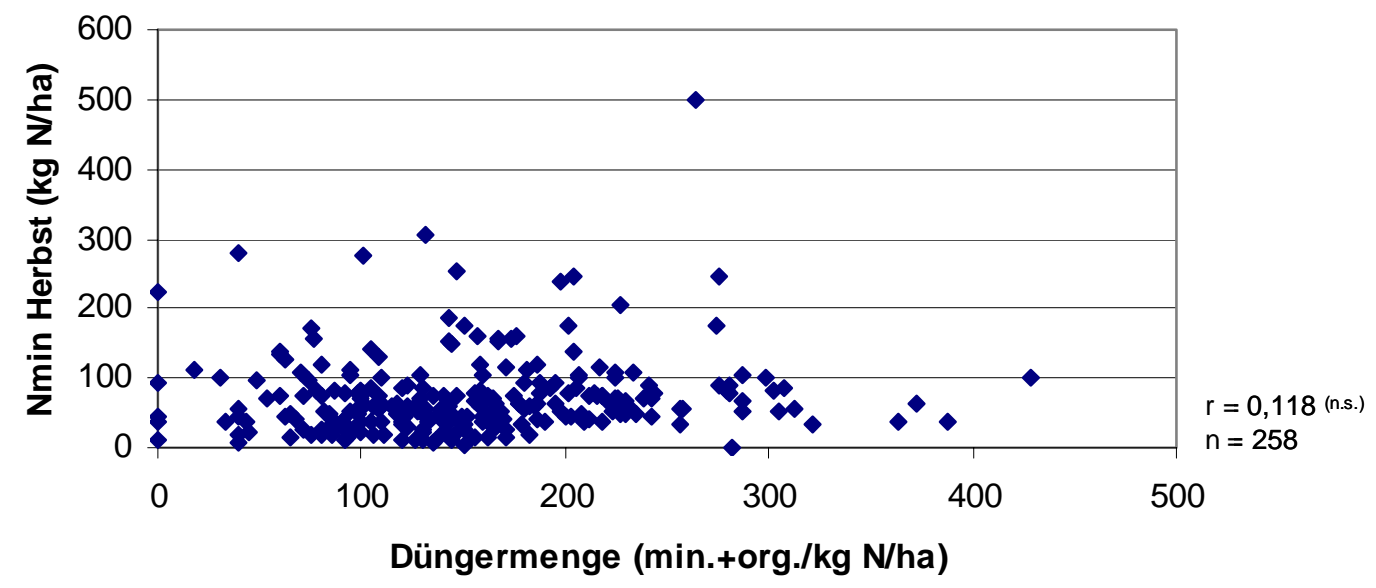

Abb. 31: Herbst $\mathrm{N}_{\min }$-Gehalt (Vegetationsende) im Verhältnis zur ausgebrachten mineralischen und organischen Düngermenge (Korrelation nach Pearson I Datenkategorie 2)

Eine weitere Differenzierung der Abb. 31 nach der Nutzungsart führt nicht zu signifikanten Zusammenhängen.

In der Tab. 13 werden die Daten nach ihrer Herkunft untergliedert. Auf Grund der unterschiedlichen Gruppengrößen werden die Fallgruppen mittels des Kruskal-Wallis Tests anhand der mittleren Ränge untersucht. Es kann nicht nachgewiesen werden, dass die Herbst $\mathrm{N}_{\min }$-Gehalte je nach Datenherkunft unterschiedlich sind. Die Düngermengen unterscheiden sich mit einem Signifikanzniveau von $<0,001$ zwischen den Datenherkünften.

Tab. 13: Mittelwert und mittlerer Rang (Kruskal-Wallis Test) der Herbst $\mathbf{N}_{\min }$-Mengen (Vegetationsende) und der N-Düngung (Datenkategorie 2)

\begin{tabular}{llccc}
\hline & Datenherkunft & $\mathbf{n}$ & Mittelwert & Mittlerer Rang \\
\hline $\mathbf{N}_{\text {min }}$-Herbst & $\mathrm{N}_{\text {mon }}$-Projekt & 159 & 67 & 133,6 \\
(Vegetationsende) & $\mathrm{VGL}$ & 75 & 68 & 128,6 \\
(kg N/ha) & Nitratrater & 37 & 104 & 161,2 \\
\hline org. + min. & $\mathrm{N}_{\text {mon }}$-Projekt & 232 & 147 & 204,7 \\
Düngermenge & VGL & 122 & 109 & 146,3 \\
(kg N/ha) & Nitratrater & 37 & 212 & 305,6 \\
\hline
\end{tabular}

Zur Prüfung des Einflusses der Bodenart auf den Zusammenhang zwischen Düngermenge und Herbst $\mathrm{N}_{\text {min }}$-Menge werden Korrelationen nach Pearson berechnet (Tab. 14). Die Bodenarten werden kategorisiert betrachtet (Anhang 6). Der berechnete Korrelationskoeffizient zeigt über alle Bodenartgruppen einen schwachen positiven Zusammenhang zwischen der Düngermenge und dem Herbst $\mathrm{N}_{\min }$-Gehalt 
auf. In Abb. 31 kann dieser Zusammenhang nicht signifikant abgesichert werden, da die zusätzlichen 6 Flächen, von denen die Bodenartgruppen nicht bekannt sind, einen zu großen Einfluss ausübten. In den einzelnen Bodenartgruppen (Tab. 14 ) sind keine signifikanten Zusammenhänge zu erkennen.

Tab. 14: Korrelation (Pearson) zwischen der N-Düngermenge (mineralisch/organisch) und dem Herbst $\mathbf{N}_{\min }$-Gehalt (Vegetationsende / 0-90cm / Datenkategorie 2)

\begin{tabular}{lcc}
\hline Bodenart (gruppiert) & Korrelationskoeffizient (r) & Anzahl (n) \\
\hline leicht & $-0,092^{(\text {n.s. })}$ & 62 \\
mittel & $0,097^{(\text {n.s. }}$ & 121 \\
schwer & $0,167^{(\text {n.s. })}$ & 69 \\
leicht / mittel / schwer & $\mathbf{0 , 1 2 4}^{*}$ & 252 \\
(zusammen) & & \\
\hline
\end{tabular}

Die Bodenartgruppen (leicht - schwer) zeigen einen Einfluss auf die ausgebrachte mineralische und organische Düngermenge. In der leichten Bodenartgruppe wurden im Mittel $181 \mathrm{~kg} \mathrm{~N} / \mathrm{ha}$, in der mittleren $142 \mathrm{~kg} \mathrm{~N} / \mathrm{ha}$ und in der schweren $114 \mathrm{~kg} \mathrm{~N} / \mathrm{ha}$ gedüngt. Dieser Unterschied ist mittels des Kruskal-Wallis Tests auf dem Niveau von $<0,001$ signifikant abzusichern.

Der Zusammenhang zwischen dem Herbst $\mathrm{N}_{\min }$-Gehalt und dem Anteil der ausgebrachten mineralischen Düngung an der gesamten Düngermenge wird in Abb. 32 verdeutlicht. Das Streudiagramm zeigt keinen Zusammenhang auf. Der Mittelwert der mineralischen Düngung liegt in Abb. 32 bei 85 kg N/ha. Auf 29 Flächen wurde keine mineralische Düngung ausgebracht.

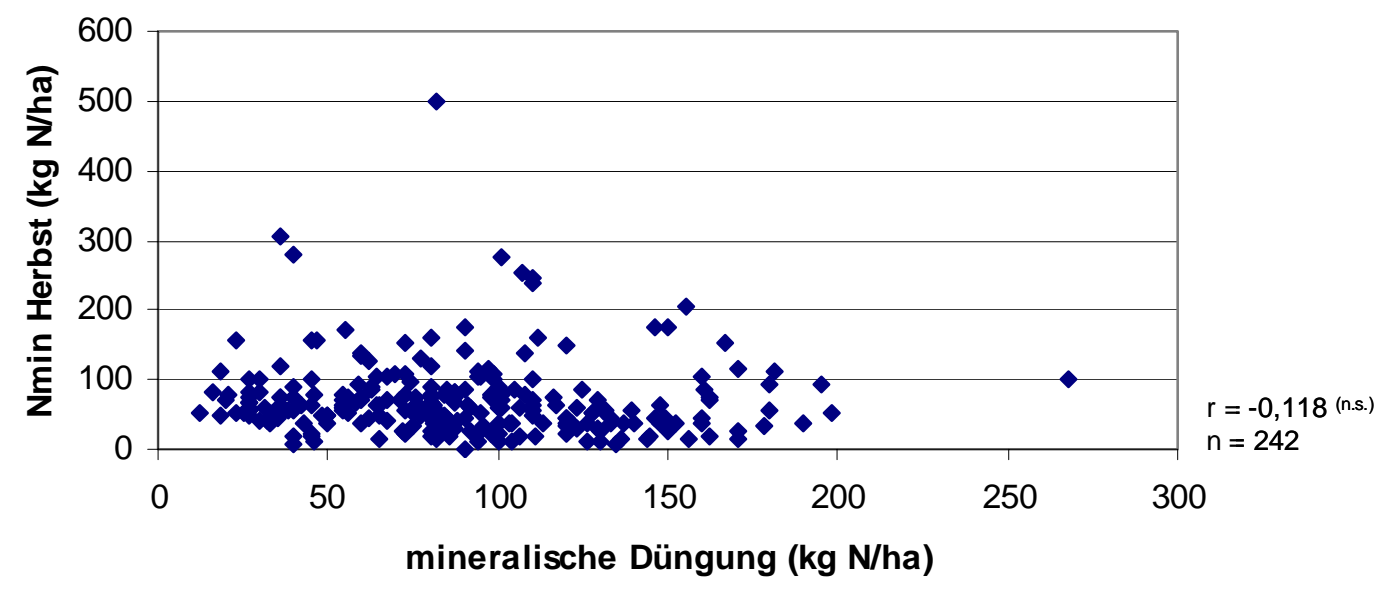

Abb. 32: Herbst $\mathrm{N}_{\min }$-Gehalte (Vegetationsende) im Verhältnis zur ausgebrachten mineralischen Düngermenge (Korrelation nach Pearson I Datenkategorie 2)

Eine Beziehung zwischen dem Viehbesatz und dem Einsatz von mineralischer Düngung besteht nicht, die Korrelation nach Pearson ist nicht signifikant. In Abb. 33 
wird die Beziehung zwischen dem Herbst $\mathrm{N}_{\min }$-Gehalt und dem Anteil der organischen Düngung an der gesamten Düngung dargestellt. Ein höherer Herbst

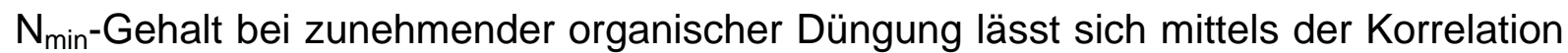
nach Pearson $(r=0,155)$ bei einer Irrtumswahrscheinlichkeit von $p=0,051$ nur in der Tendenz bestätigen. Im Mittel wurden $113 \mathrm{~kg} \mathrm{~N} / \mathrm{ha}$ an organischer Düngung appliziert. Auf 136 Flächen wurden keine organischen Dünger ausgebracht.

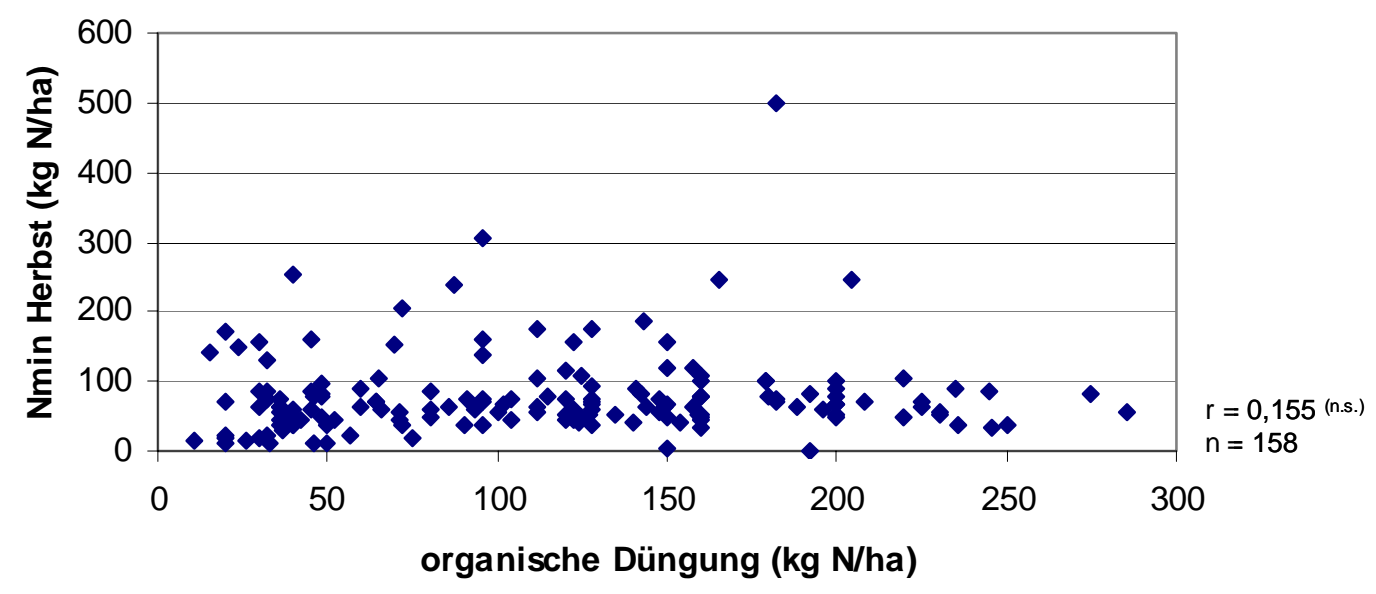

Abb. 33: Herbst $\mathrm{N}_{\min }$-Gehalte (Vegetationsende) im Verhältnis zur ausgebrachten organischen Düngermenge (Korrelation nach Pearson / Datenkategorie 2)

Dass der Viehbesatz einen Einfluss $(r=0,427)$ auf die organische Düngung hat, konnte mittels Korrelation nach Pearson auf 30 Flächen mit einer Irrtumswahrscheinlichkeit von $p=0,019$ bestätigt werden. Zwischen den Viehbesatzdichten und den $\mathrm{N}_{\text {min }}$-Mengen im Frühjahr und Herbst ist kein Zusammenhang festzustellen.

Auf den Flächen, die nur mineralisch (160 Flächen) oder organisch (25 Flächen) gedüngt wurden, zeigen sich ebenfalls keine korrelativen Beziehungen zum Herbst $\mathrm{N}_{\text {min- }}$-Gehalt. 190 Flächen wurden kombiniert gedüngt und auf 39 Flächen wurde keine Düngung appliziert (Anhang 20).

Der Zusammenhang zwischen $\mathrm{N}$-Saldo und Herbst $\mathrm{N}_{\min }$-Menge ist in der Abb. 34 dargestellt. Es besteht keine Beziehung zwischen dem Herbst $\mathrm{N}_{\text {min }}$-Gehalt und dem $\mathrm{N}$-Saldo. Der Mittelwert des N-Saldos liegt in der Abb. 34 bei $-23 \mathrm{~kg} \mathrm{~N} / \mathrm{ha}$. Eine weitere Differenzierung nach Bodenartgruppen (leicht - schwer) führt auch nicht zu signifikanten Korrelationen zwischen dem N-Saldo und dem Herbst $\mathrm{N}_{\min }$-Gehalt. 


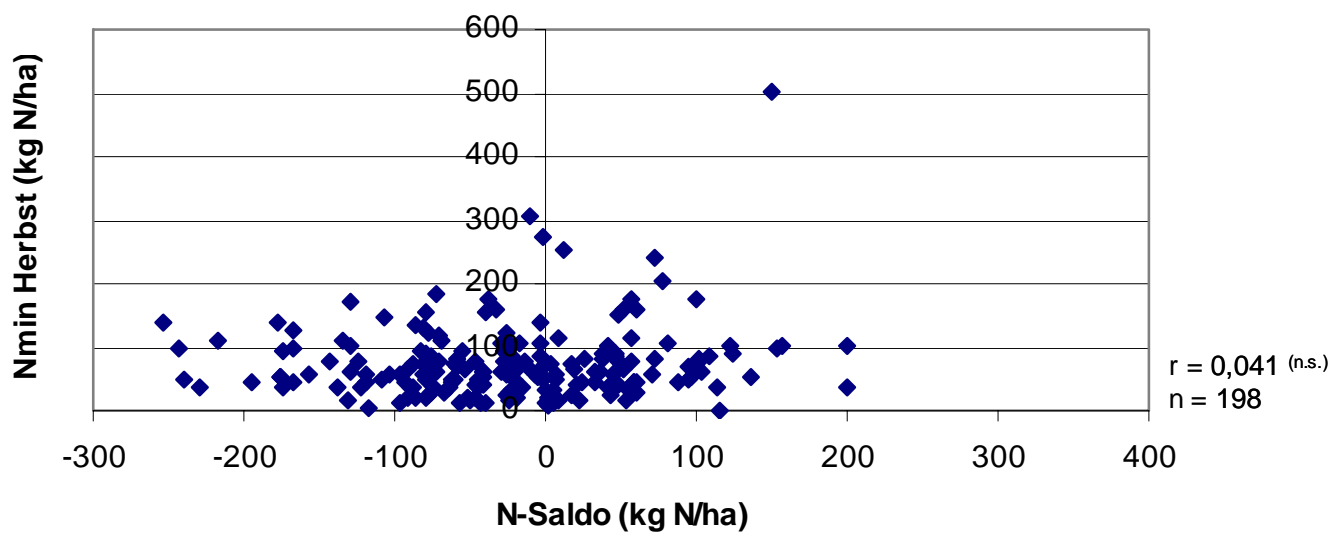

Abb. 34: Herbst $\mathrm{N}_{\min }$-Gehalte (Vegetationsende) im Verhältnis zum N-Saldo (Korrelation nach Pearson / Datenkategorie2)

Der Einfluss der gebildeten Bodenartgruppen (leicht - schwer) auf den Herbst $\mathrm{N}_{\text {min }}{ }^{-}$ Gehalt ist mit dem Kruskal-Wallis Test untersucht worden. Es besteht ein signifikanter Unterschied $(p=0,008)$ zwischen den Bodenartgruppen und dem Herbst $\mathrm{N}_{\min }$-Gehalt.

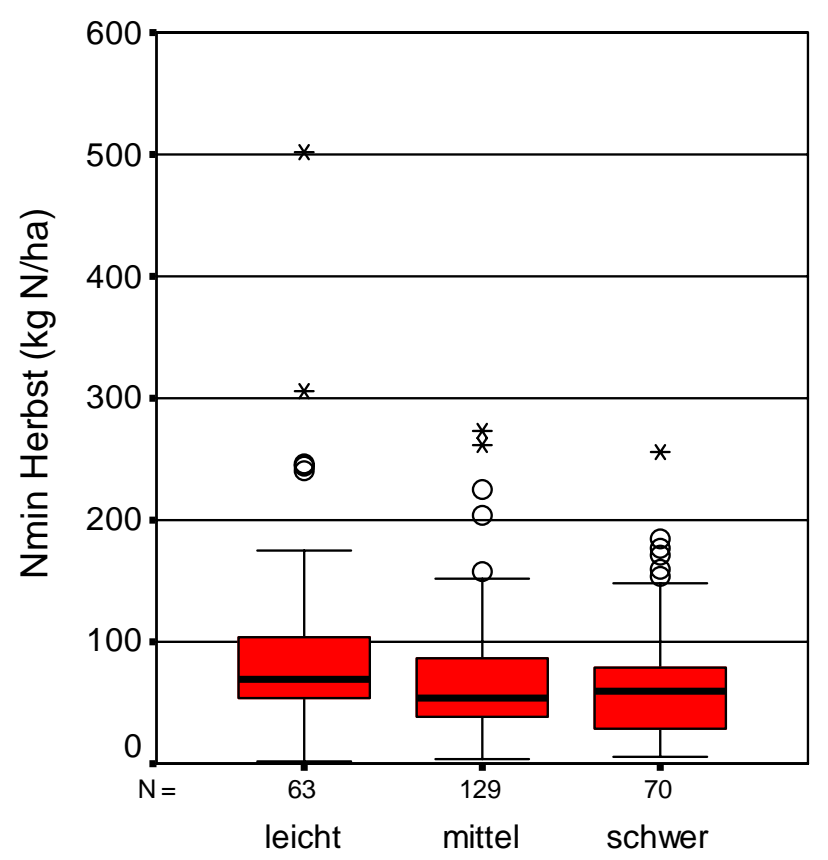

Abb. 35: Herbst $\mathrm{N}_{\min }$-Mengen (kg N/ha I Vegetationsende) je nach Bodenartgruppe (Datenkategorie 2)

In der Abb. 36 wird die Beziehung zwischen dem C/N-Verhältnis und dem Herbst $\mathrm{N}_{\text {min }}$-Gehalt dargestellt. Da im Vergleichsflächenprogramm kein $\mathrm{C} / \mathrm{N}$-Verhältnis des Bodens ermittelt worden ist, bezieht sich die folgende Grafik nur auf die Daten aus 
dem $\mathrm{N}_{\text {mon-Projekt }}$ und dem Nitratraster. Eine Beziehung zwischen dem $\mathrm{C} / \mathrm{N}$ Verhältnis und der Herbst $\mathrm{N}_{\min }$-Menge kann nicht festgestellt werden. Bei einer

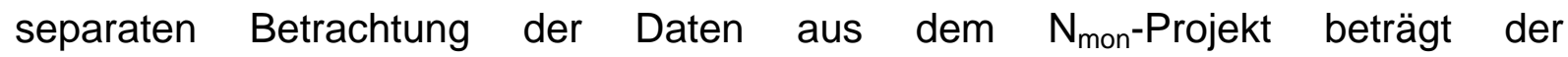
Korrelationskoeffizient nach Pearson -0,224 bei einem Signifikanzniveau von 0,011. Dieses bedeutet, dass der Herbst $\mathrm{N}_{\min }$-Gehalt mit enger werdendem C/N-Verhältnis steigt.

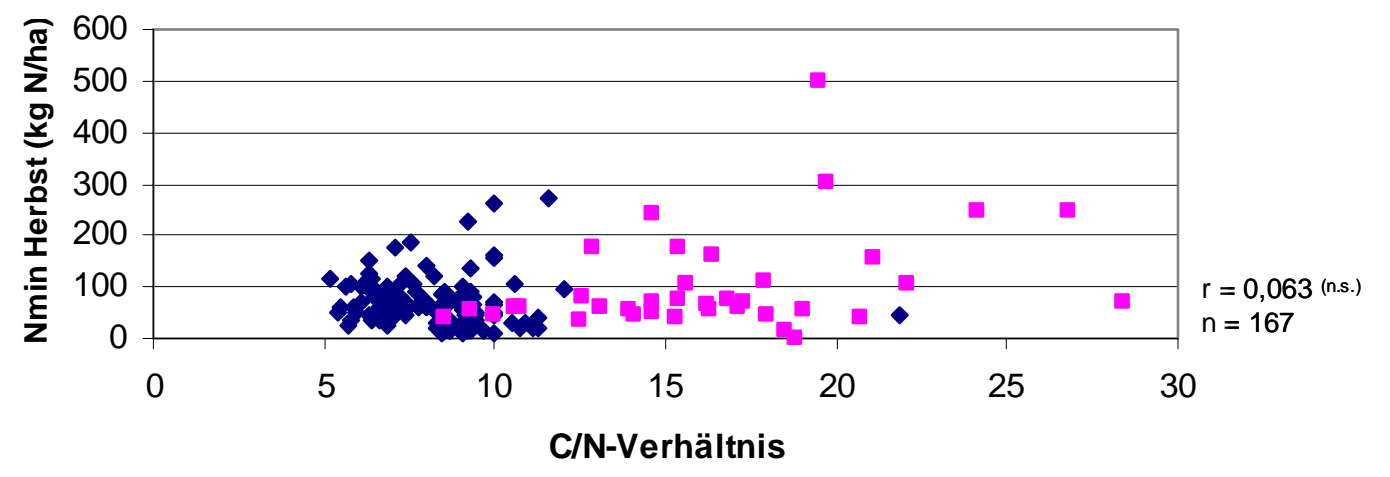

- Nmon-Projekt $\approx$ Nitratraster

Abb. 36: Herbst $\mathrm{N}_{\min }$-Gehalte (Vegetationsende) im Verhältnis zum $\mathrm{C} / \mathrm{N}-$ Verhältnis des Bodens (Korrelation nach Pearson / Datenkategorie 2)

Eine Untersuchung der Beziehung zwischen dem Viehbesatz und dem $\mathrm{C} / \mathrm{N}$ Verhältnis ist aus Ermangelung an Daten nicht möglich. Ein Einfluss der organischen Düngung auf das C/N-Verhältnis kann mittels der Korrelationsanalyse nach Pearson nicht festgestellt werden. Ob das C/N-Verhältnis eine Abhängigkeit mit den gebildeten Bodenartgruppen aufweist, ist mit dem Kruskal-Wallis Test untersucht worden. So kann mit einem Signifikanzniveau von <0,001 davon ausgegangen werden, dass das $\mathrm{C} / \mathrm{N}-$ Verhältnis je nach Bodengruppe unterschiedlich ist. Die Bodenartgruppen mit mehr als 12\% Tonanteil weisen engere $\mathrm{C} / \mathrm{N}$-Verhältnisse auf als die leichte Bodenartgruppe.

Die Beziehung zwischen dem Gesamt-N Gehalt und der Herbst $\mathrm{N}_{\min }$-Menge (Abb. 37) ist mit einem Korrelationskoeffizienten nach Pearson von 0,240 auf dem Niveau von 0,002 signifikant. Bei einer Untergliederung nach den gebildeten Bodenartgruppen hebt sich besonders die mittlere Fraktion (12-25\% Tonanteil) mit einem Korrelationskoeffizienten von 0,395 bei einem Signifikanzniveau von $<0,001$ hervor. 


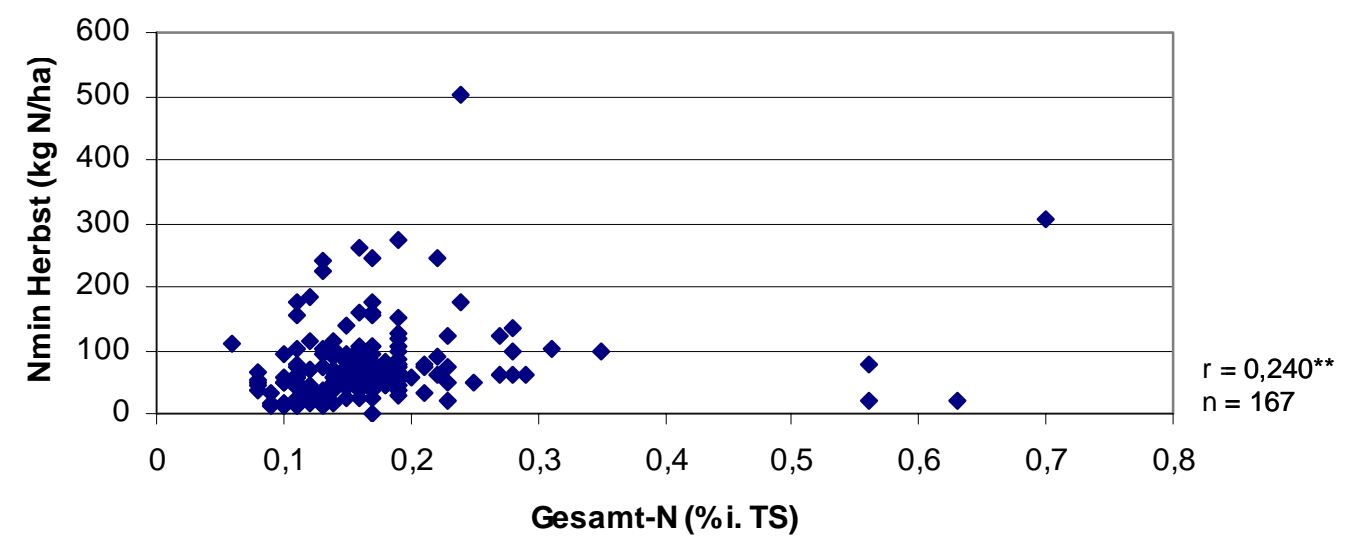

Abb. 37: Herbst $\mathrm{N}_{\min }$-Gehalte (Vegetationsende) im Verhältnis zum Gesamt-N Gehalt des Bodens (Korrelation nach Pearson I Datenkategorie 2)

Eine Beziehung zwischen der ausgebrachten organischen Düngermenge und dem Gesamt-N Gehalt des Bodens kann nicht festgestellt werden. Der Einfluss der Viehbesatzdichte auf den Gesamt-N Gehalt des Bodens kann aus Ermangelung an zur Verfügung stehenden Daten nicht untersucht werden. Der Gesamt-N Gehalt des Bodens nimmt mit dem Tonanteil im Boden signifikant zu. Dies bedeutet, dass die Bodenartgruppe mit einem Tonanteil von kleiner 12\% den niedrigsten Mittelwert mit 0,15\% Gesamt-N i. TS und die Bodenartgruppe mit mehr als 25\% Tonanteil den höchsten Mittelwert mit 0,20\% Gesamt-N i. TS aufweist.

Zwischen den Herbst $\mathrm{N}_{\min }$-Mengen und den $\mathrm{C}_{\mathrm{t}}$-Gehalten besteht ein schwacher Zusammenhang (Abb. 38). Die Korrelation nach Pearson weist einen Korrelationskoeffizienten von 0,191 bei einem Signifikanzniveau von 0,004 aus. Die Streuung nimmt mit steigenden $\mathrm{C}_{\mathrm{t}}$-Gehalten des Bodens zu. Dieses bedeutet, dass auf Standorten mit höherem Humusgehalt die Abschätzung von Herbst $\mathrm{N}_{\text {min }^{-}}$ Gehalten erheblich schwerer fällt. Durch die Gruppierung nach den drei Bodenartgruppen können keine deutlich höheren Korrelationskoeffizienten erzielt werden. 


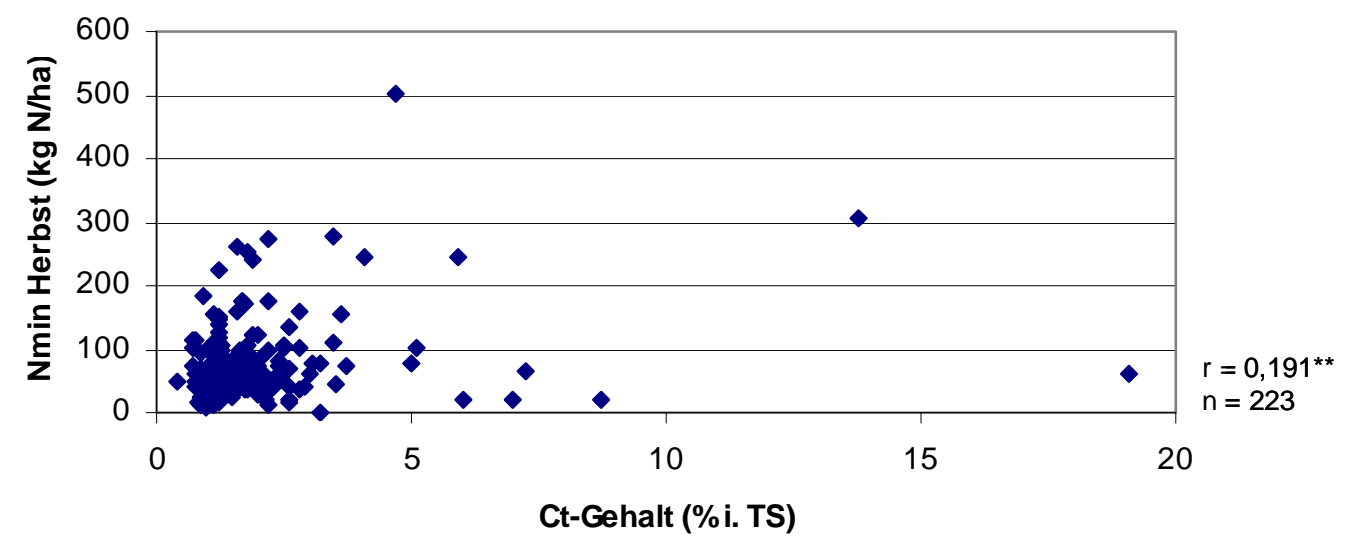

Abb. 38: Herbst $\mathrm{N}_{\min }$-Gehalt (Vegetationsende) im Verhältnis zum $\mathrm{C}_{\mathrm{t}}$-Gehalt des Bodens (Korrelation nach Pearson I Datenkategorie 2)

Eine Beziehung zwischen der ausgebrachten organischen Düngermenge und dem $\mathrm{C}_{\mathrm{t}}$-Gehalt des Bodens kann nicht festgestellt werden. Auch der Viehbesatz zeigt keinen Einfluss auf den $\mathrm{C}_{\mathrm{t}}$-Gehalt des Bodens. Die Differenzierung der $\mathrm{C}_{\mathrm{t}}$-Gehalte nach Bodenarten zeigt keine eindeutige Tendenz.

Die Korrelation der Frühjahrs $\mathrm{N}_{\min }$-Gehalte und der folgenden Herbst $\mathrm{N}_{\min }$-Gehalte zeigt die Tab. 15. Des Weiteren werden in der Tabelle die Zusammenhänge der Herbst $\mathrm{N}_{\text {min }}$-Menge direkt nach der Ernte und vor Vegetationsende mit der Korrelationsanalyse untersucht. Die Korrelationen werden in Abhängigkeit von den drei gebildeten Bodenartgruppen ermittelt. Wie aus der Tabelle hervorgeht, stehen die Frühjahrs und Herbst $\mathrm{N}_{\min }$-Gehalte in einer Beziehung. Der Herbst $\mathrm{N}_{\min }$-Gehalt nach der Ernte und vor Vegetationsende weist eine relativ enge Korrelation auf. 
Tab. 15: Korrelation (Pearson) zwischen dem Frühjahrs und dem nachfolgenden Herbst $\mathbf{N}_{\min }{ }^{-}$ Gehalt (0-90cm I Datenkategorie 2)

\begin{tabular}{|c|c|c|c|}
\hline Bodengruppe & $\mathbf{N}_{\min }$ Termin & $\mathbf{N}_{\min }$ Frühjahr & $\begin{array}{r}\mathrm{N}_{\min } \text { Herbst } \\
\text { (nach Ernte) }\end{array}$ \\
\hline $\begin{array}{l}\text { Leicht } \\
(<12 \%\end{array}$ & $\begin{array}{l}\mathbf{N}_{\min } \text { Herbst } \\
\text { (nach Ernte) }\end{array}$ & $\begin{array}{c}-, 133^{(\text {n.s. })} \\
(n=23)\end{array}$ & - \\
\hline Tonanteil) & $\begin{array}{l}\mathrm{N}_{\min } \text { Herbst } \\
\text { (Vegetationsende) }\end{array}$ & $\begin{array}{l}, 627^{\star *} \\
(n=48)\end{array}$ & $\begin{array}{l}, 698^{* *} \\
(\mathrm{n}=27)\end{array}$ \\
\hline $\begin{array}{l}\text { Mittel } \\
(12-25 \%\end{array}$ & $\begin{array}{l}\mathrm{N}_{\min } \text { Herbst } \\
\text { (nach Ernte) }\end{array}$ & $\begin{array}{l}, 378^{* *} \\
(n=123)\end{array}$ & - \\
\hline Tonanteil) & $\begin{array}{l}\mathrm{N}_{\min } \text { Herbst } \\
\text { (Vegetationsende) }\end{array}$ & $\begin{array}{c}, 498 * * \\
(n=119)\end{array}$ & $\begin{array}{l}, 736 * * \\
(n=95)\end{array}$ \\
\hline $\begin{array}{l}\text { Schwer } \\
(>25 \%\end{array}$ & $\begin{array}{l}N_{\min } \text { Herbst } \\
\text { (nach Ernte) }\end{array}$ & $\begin{array}{l}, 521^{* *} \\
(\mathrm{n}=57)\end{array}$ & - \\
\hline Tonanteil) & $\begin{array}{l}\mathrm{N}_{\min } \text { Herbst } \\
\text { (Vegetationsende) }\end{array}$ & $\begin{array}{l}, 448^{* *} \\
(n=66)\end{array}$ & $\begin{array}{l}, 778^{* *} \\
(\mathrm{n}=49)\end{array}$ \\
\hline $\begin{array}{l}\text { (Leicht, } \\
\text { Mittel, }\end{array}$ & $\begin{array}{l}\mathrm{N}_{\min } \text { Herbst } \\
\text { (nach Ernte) }\end{array}$ & $\begin{array}{c}, 340 * * \\
(n=203)\end{array}$ & \\
\hline Schwer) & $\begin{array}{l}\mathrm{N}_{\min } \text { Herbst } \\
\text { (Vegetationsende) }\end{array}$ & $\begin{array}{c}, 483^{* *} \\
(n=233)\end{array}$ & $\begin{array}{c}, 736 * * \\
(\mathrm{n}=171)\end{array}$ \\
\hline
\end{tabular}

In Abb. 39 ist der Einfluss der Ackerzahl auf die Herbst $\mathrm{N}_{\min }$-Menge dargestellt. Eine Beziehung zwischen der Ackerzahl und den Herbst $\mathrm{N}_{\min }$-Mengen kann nicht festgestellt werden.

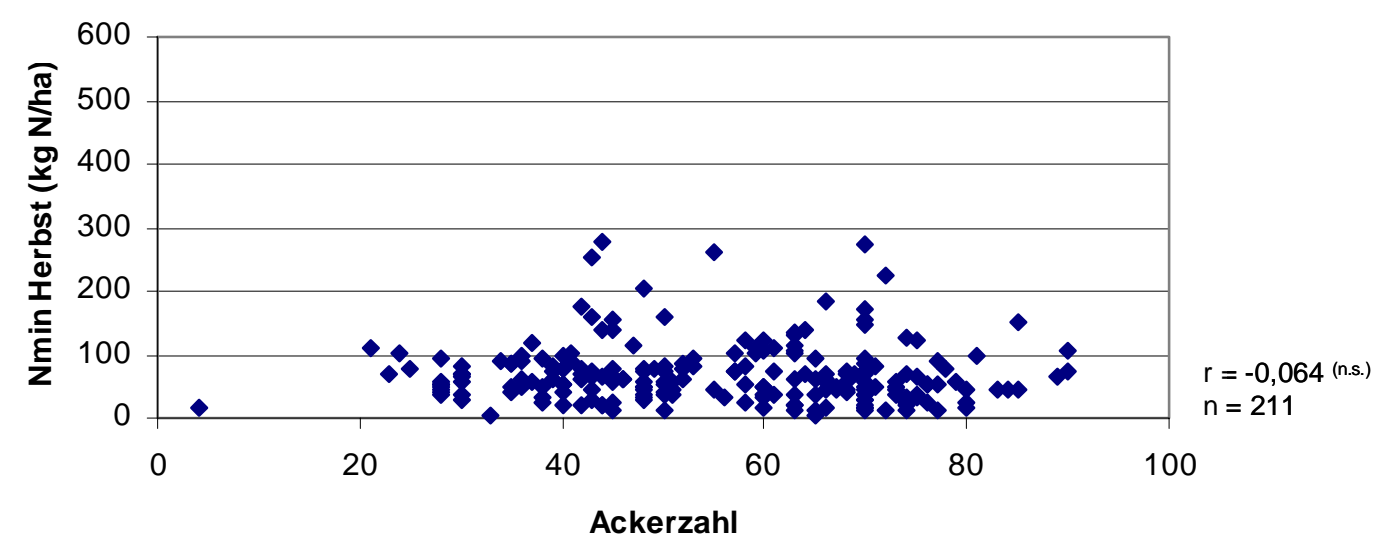

Abb. 39: Herbst $\mathrm{N}_{\min }$-Gehalt (Vegetationsende) im Verhältnis zur Ackerzahl (Korrelation nach Pearson / Datenkategorie 2)

Eine Beziehung zwischen dem $\mathrm{pH}$-Wert des Bodens und dem $\mathrm{N}_{\min }$-Gehalt im Herbst ist mittels der Korrelation nach Pearson nicht nachzuweisen. Die $\mathrm{P}_{2} \mathrm{O}_{5}$ Gehalte des Bodens zeigen ebenfalls keinen Einfluss auf den Herbst $\mathrm{N}_{\min }$-Gehalt.

Die bisher mit Korrelationen untersuchten Zusammenhänge zwischen der abhängigen Variable Herbst $\mathrm{N}_{\min }$-Gehalt und den unabhängigen Variablen werden nun mittels der multiplen Regression untersucht. Die multiple Regression wird deshalb eingesetzt, weil der Einfluss eines Faktors in den Korrelationen durch einen 
anderen verschleiert werden kann. In die schrittweise multiple Regression sind die folgenden unabhängigen Variablen eingeflossen: $\mathrm{N}_{\text {min }}$-Frühjahr, Gesamt-N Gehalt, $\mathrm{C}_{\mathrm{t}}-\mathrm{Gehalt}, \mathrm{C} / \mathrm{N}-$ Verhältnis, organische Düngung, mineralische Düngung, N-Saldo und die Ackerzahl. Die Regression weist mit einem $r^{2}$-Wert von 0,364 eine Beziehung zwischen der abhängigen Variable und den unabhängigen Variablen (organische Düngermenge / Gesamt-N Gehalt) nach.

Tab. 16: Multiple Regression mit dem Herbst $\mathrm{N}_{\min }$-Gehalt (Vegetationsende) als abhängige Variable (Regressor / Datenkategorie 2)

\begin{tabular}{|c|c|c|c|c|}
\hline & b & SF & $t$ & p \\
\hline Konstante & 3,628 & 0,773 & 4,694 & $<0,001$ \\
\hline $\begin{array}{l}\text { Düngermenge } \\
\text { (organisch) }\end{array}$ & 0,354 & 0,094 & 3,762 & $<0,001$ \\
\hline $\begin{array}{l}\text { Gesamt-N Gehalt } \\
\text { (Boden) }\end{array}$ & 0,632 & 0,262 & 2,410 & 0,019 \\
\hline
\end{tabular}

$r^{2}=\mathbf{0 , 3 6 5} \quad \mathrm{F}=16,67 \quad \mathrm{p}<0,001 \quad \mathrm{n}=61$

\section{Einfluss der Vor- und Zwischenfrüchte auf den Frühjahrs und Herbst $\mathrm{N}_{\text {min}^{-}}$} Gehalt

Der Zwischenfruchtanbau vor dem Maisanbau wird in dieser Datenkategorie in „Brache“, „Leguminose“ oder "Nicht Leguminose“ unterschieden, da diese Vorgabe durch die Daten aus dem $\mathrm{N}_{\text {mon }}$-Projekt gegeben ist. In der Fraktion "Leguminose“ steht nur eine Fläche für Auswertungen zur Verfügung; diese Gruppe wird daher nicht weiter berücksichtigt. Ein sich anschließender Vergleich mittels des t-Tests zwischen Brache und einer Bodenbedeckung (Nicht Leguminose) zeigt, dass sich die Mittelwerte der Herbst $\mathrm{N}_{\min }$-Mengen mit einer Irrtumswahrscheinlichkeit von $\mathrm{p}=0,045$ unterscheiden. So sind die Herbst $\mathrm{N}_{\text {min }}$-Mengen bei dem Anbau einer Zwischenfrucht vor Mais niedriger. Ein ähnlicher Unterschied kann bei den Frühjahrs $\mathrm{N}_{\min }$-Mengen nicht festgestellt werden. 

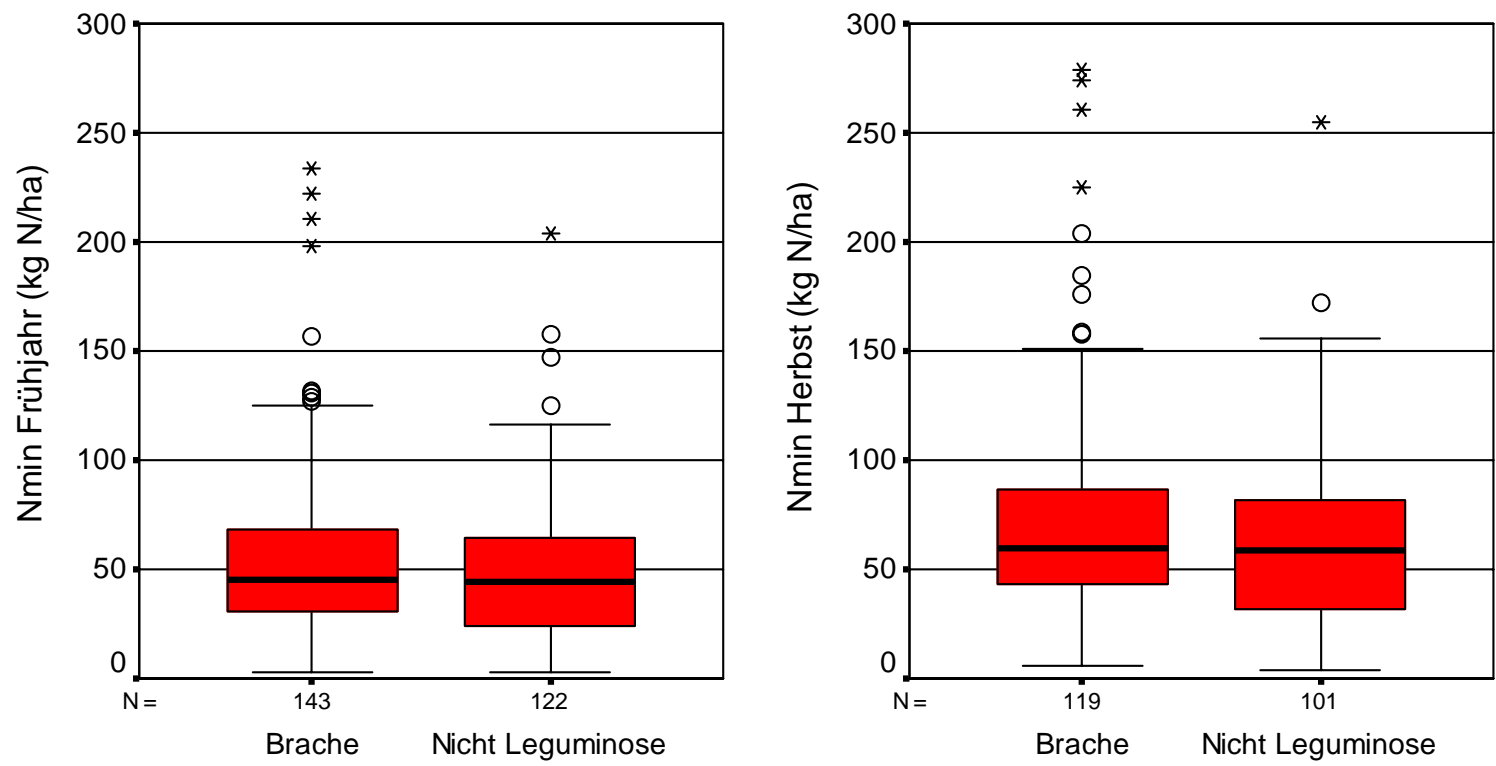

Abb. 40: Verteilung der Frühjahrs und Herbst $\mathrm{N}_{\min }$-Gehalte ( $\mathrm{kg} \mathrm{N} / \mathrm{ha} /$ Vegetationsende) in Abhängigkeit von der „Brache“ oder dem Einsatz einer „Nicht Leguminosen“ Zwischenfrucht vor dem Maisanbau (Datenkategorie 2)

$\mathrm{Ob}$ die ausgebrachte Düngermenge zum Mais in Abhängigkeit von der Zwischenfrucht variiert und damit einen Einfluss auf den Herbst $\mathrm{N}_{\min }$-Gehalt hat, wird im Weiteren untersucht. Die zu Mais ausgebrachte Düngermenge ist bei Flächen mit einem Zwischenfruchtanbau vor Mais um $30 \mathrm{~kg}$ N/ha niedriger als bei den Flächen, die brach lagen. Dieser Unterschied lässt sich durch den Mann-Whitney Test mit einem Signifikanzniveau von $<0,001$ belegen. Es wurde auf Flächen, die Zwischenfrüchte vor dem Mais aufwiesen, im Mittel $129 \mathrm{~kg} \mathrm{~N} / \mathrm{ha}$ und auf Flächen die vorher brach lagen $159 \mathrm{~kg} \mathrm{~N} / \mathrm{ha}$ ausgebracht. Die Anzahl der Daten in dieser Berechnung ist leicht abweichend zu der Darstellung mit den Herbst $\mathrm{N}_{\min }$-Gehalten in Abb. 40, da nicht von jeder Fläche die Düngermenge bekannt ist. Insgesamt liegen von 110 Flächen mit Brache und 100 Flächen mit einer nicht leguminosen Zwischenfrucht die Düngermengen vor.

Der Einfluss der Vorfrucht auf die Frühjahrs und Herbst $\mathrm{N}_{\min }$-Gehalte wird mittels Boxplot in Abb. 41 dargestellt. Auf Grund der Vielzahl an Vorfrüchten werden nur jene dargestellt, die mindestens auf 2\% der Flächen vorkamen. Da trotz dieser Einschränkung einige Vorfrüchte nur auf 7 Flächen vorhanden waren, wird auf eine statistische Untersuchung verzichtet, da die meisten dafür geeigneten Analysen die Prozedur aus Ermangelung an Daten abbrechen. So zeigen die Boxplots aber auch 
hier, dass der in Selbstfolge stehende Mais nicht besonders hohe Rest $\mathrm{N}_{\min }$-Gehalte hinterlässt. Zu Vegetationsbeginn weist nur der Winterraps höhere $\mathrm{N}_{\min }$-Gehalte auf.
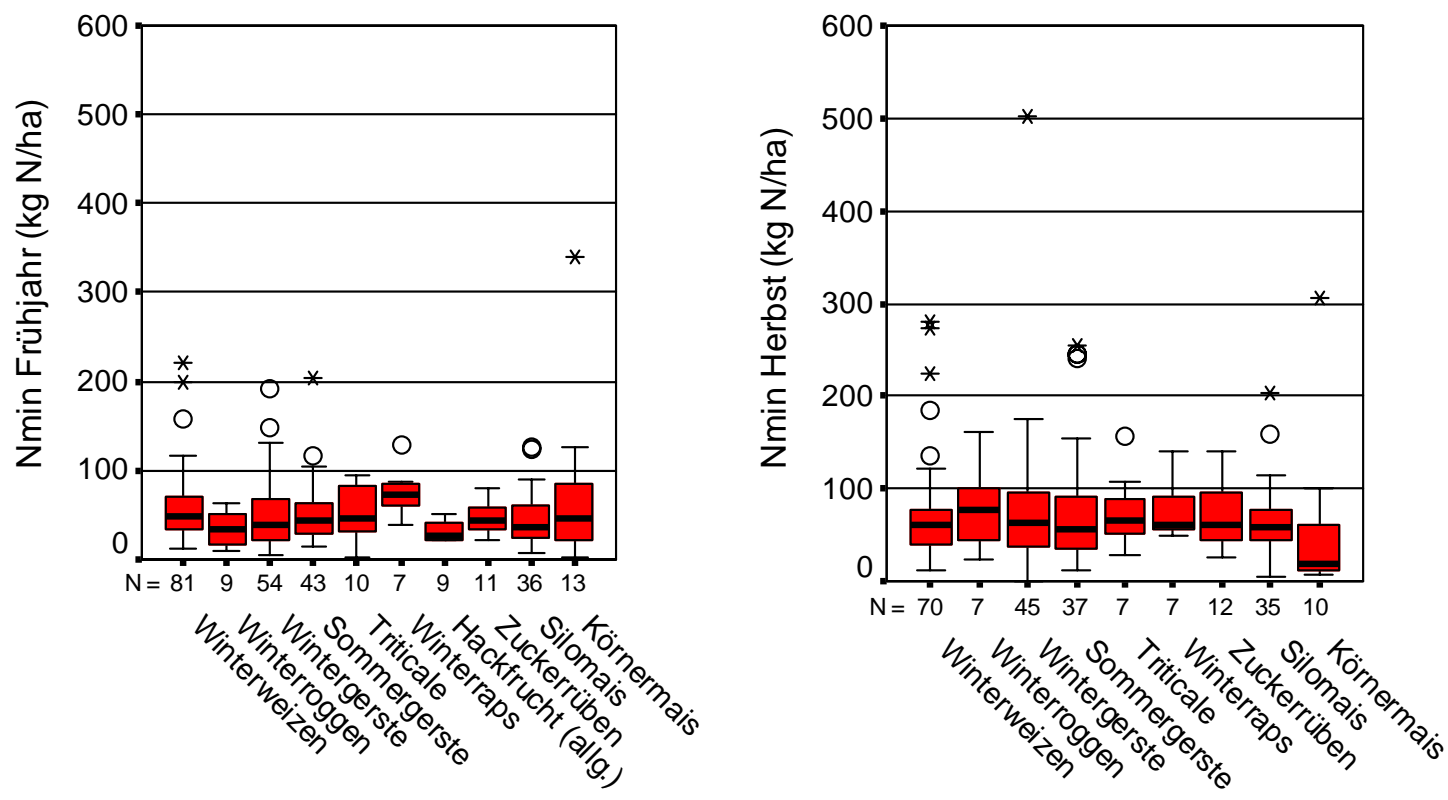

Abb. 41: Verteilung der Frühjahrs und Herbst $\mathrm{N}_{\min }$-Gehalte ( $\mathrm{kg} \mathrm{N} / \mathrm{ha} /$ Vegetationsende) in Abhängigkeit von der Vorfrucht vor dem Maisanbau (Datenkategorie 2)

\section{Einfluss der Witterung auf den Herbst $\mathrm{N}_{\min }$-Gehalt}

Tab. 17 zeigt den Einfluss der Witterung auf die Ausprägung der Herbst $\mathrm{N}_{\text {min- }}$ Mengen. In der Tab. 17 zeigt sich ein durchgehender schwacher Einfluss der Temperatur vor der Herbst $\mathrm{N}_{\min }$-Beprobung auf den Herbst $\mathrm{N}_{\min }$-Gehalt. So nehmen die Herbst $\mathrm{N}_{\min }$-Gehalte mit steigender Temperatur ab. Der Niederschlag in den Monaten vor der Herbst $\mathrm{N}_{\min }$-Beprobung steht in einigen Monaten ebenfalls in schwacher Beziehung zum Herbst $\mathrm{N}_{\min }$-Gehalt. Dieser nimmt mit zunehmendem Niederschlag ab. Der negative Korrelationskoeffizient $(r=-0,203 / p<0,001)$ zwischen dem Herbst $\mathrm{N}_{\min }$-Gehalt und der Summe der Niederschläge von Mai bis einschließlich September bestätigt diese Feststellung.

Tab. 17: Korrelation (Kendall) zwischen der Witterung (Temperatur und Niederschlag) und dem Herbst $\mathrm{N}_{\min }$-Gehalt (0-90cm / Vegetationsende / Datenkategorie 2)

\begin{tabular}{lllcccc}
\hline & & \multicolumn{5}{c}{ Monat vor der Beprobung } \\
& Mai & Juni & Juli & August & September & Oktober \\
\hline Temperatur &,,$- 157^{* *}$ &,$- 160^{* *}$ &,$- 091^{*}$ &,$- 153^{* *}$ &,$- 053^{* *}$ &,$- 139^{* *}$ \\
& $(n=255)$ & $(n=255)$ & $(n=254)$ & $(n=254)$ & $(n=254)$ & $(n=212)$ \\
Niederschlag &,$- 185^{* *}$ &, $007^{(n . s .)}$ &,$- 110^{* *}$ &,$- 104^{*}$ &,$- 193^{* *}$ &,$- 048^{(n . s .)}$ \\
& $(n=255)$ & $(n=255)$ & $(n=254)$ & $(n=254)$ & $(n=254)$ & $(n=212)$ \\
\hline
\end{tabular}




\subsection{Auswertung der Daten aus Exaktversuchen (Datenkategorie 3)}

\subsubsection{Variabilität der untersuchten Merkmale}

Für die Auswertung der Daten aus den Exaktversuchen stehen je nach Merkmal 13 bis 135 Werte aus den zufällig ausgewählten Null- und Düngeparzellen zur Verfügung. Zunächst wird die Variabilität der Merkmale dargestellt. Auf den Nullparzellen (ohne Düngung / Abb. 42) werden im Mittel Herbst $\mathrm{N}_{\text {min }}$-Mengen von 33 $\mathrm{kg} \mathrm{N} / \mathrm{ha}$ im Bodenprofil 0 bis $90 \mathrm{~cm}$ festgestellt. Die gedüngten Parzellen (mit Düngung / Abb. 42) weisen im Mittel eine Herbst $\mathrm{N}_{\min }$-Menge von $55 \mathrm{~kg} \mathrm{~N} / \mathrm{ha}$ (0-90 $\mathrm{cm}$ ) auf. Die $\mathrm{N}_{\min }$-Beprobung im Herbst fand zu über 95\% direkt nach der Ernte statt.
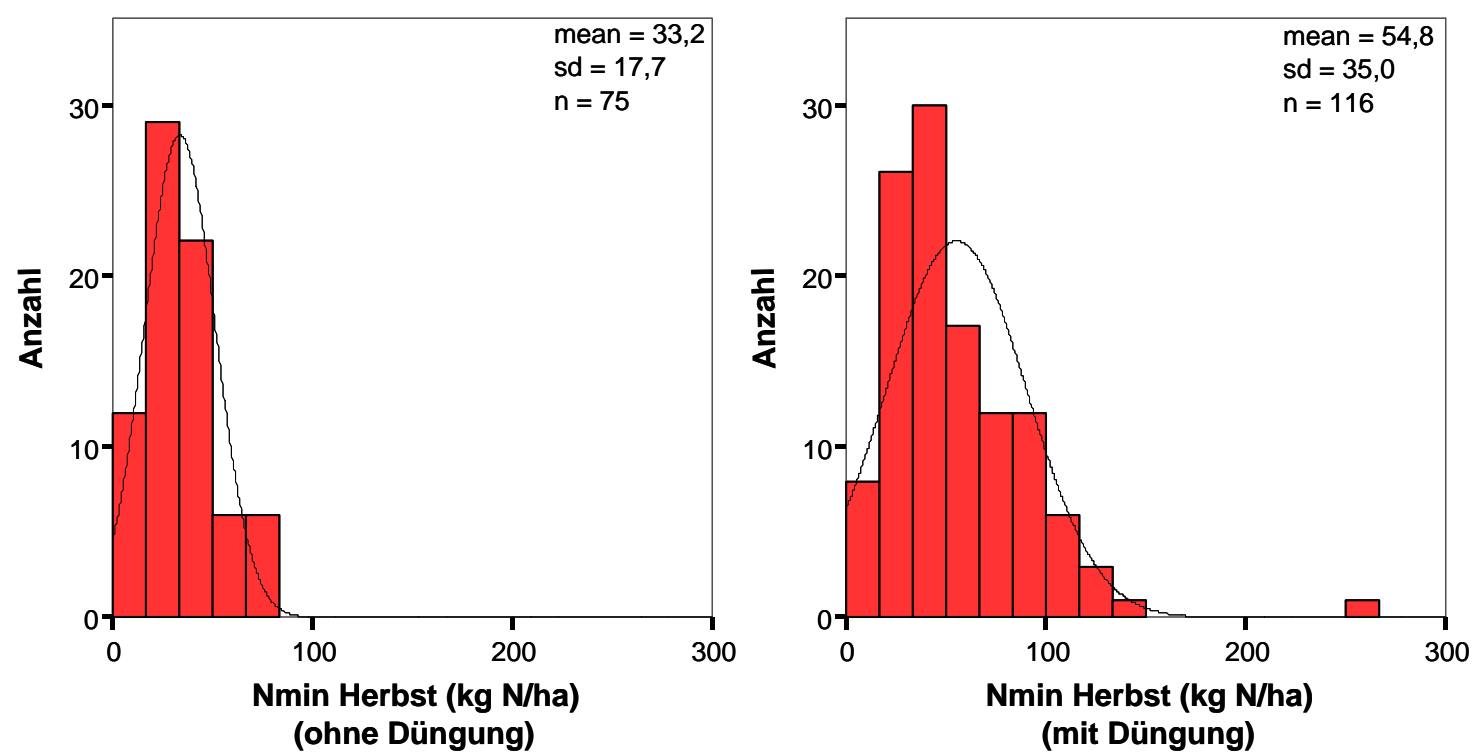

Abb. 42: Häufigkeitsverteilung der Herbst $\mathrm{N}_{\min }$-Gehalte $(0-90 \mathrm{~cm})$ nach der Ernte (Datenkategorie 3)

Die Frühjahrs $\mathrm{N}_{\min }$-Gehalte wurden über einen relativ weiten Beprobungszeitraum ermittelt. Es liegen nahezu 50\% der Beprobungen vor dem ersten Mai. Die weiteren $50 \%$ der Beprobungen wurden dann Größtenteils ab dem 15. Mai vorgenommen. Auf 23 ungedüngten Flächen und auf 53 gedüngten Flächen fand eine Frühjahrs $\mathrm{N}_{\text {min }}{ }^{-}$ Beprobung vor dem ersten Mai statt (Anhang 15). Die Mittelwerte liegen bei den nicht gedüngten Flächen bei $55 \mathrm{~kg} \mathrm{~N}$ min $/$ ha und bei den gedüngten Flächen bei $62 \mathrm{~kg}$ $\mathrm{N}_{\text {min }} /$ ha. 27 ungedüngte Flächen und 54 gedüngte Flächen wurden ab dem ersten Mai beprobt. Im Mittel werden auf den ungedüngten Flächen $94 \mathrm{~kg} \mathrm{~N} \mathrm{Nin}_{1} / \mathrm{ha}$ und auf den gedüngten Flächen $90 \mathrm{~kg} \mathrm{~N}$ min $/$ ha gefunden. In der Abb. 43 werden alle 
vorliegenden Frühjahrs $\mathrm{N}_{\min }$-Gehalte der gedüngten und ungedüngten Flächen gezeigt. Die Anzahl der eingeflossen Frühjahrs $\mathrm{N}_{\text {min }}$-Gehalte erhöht sich in diesem Histogramm, da nicht von jeder Beprobung ein exaktes Datum vorliegt.
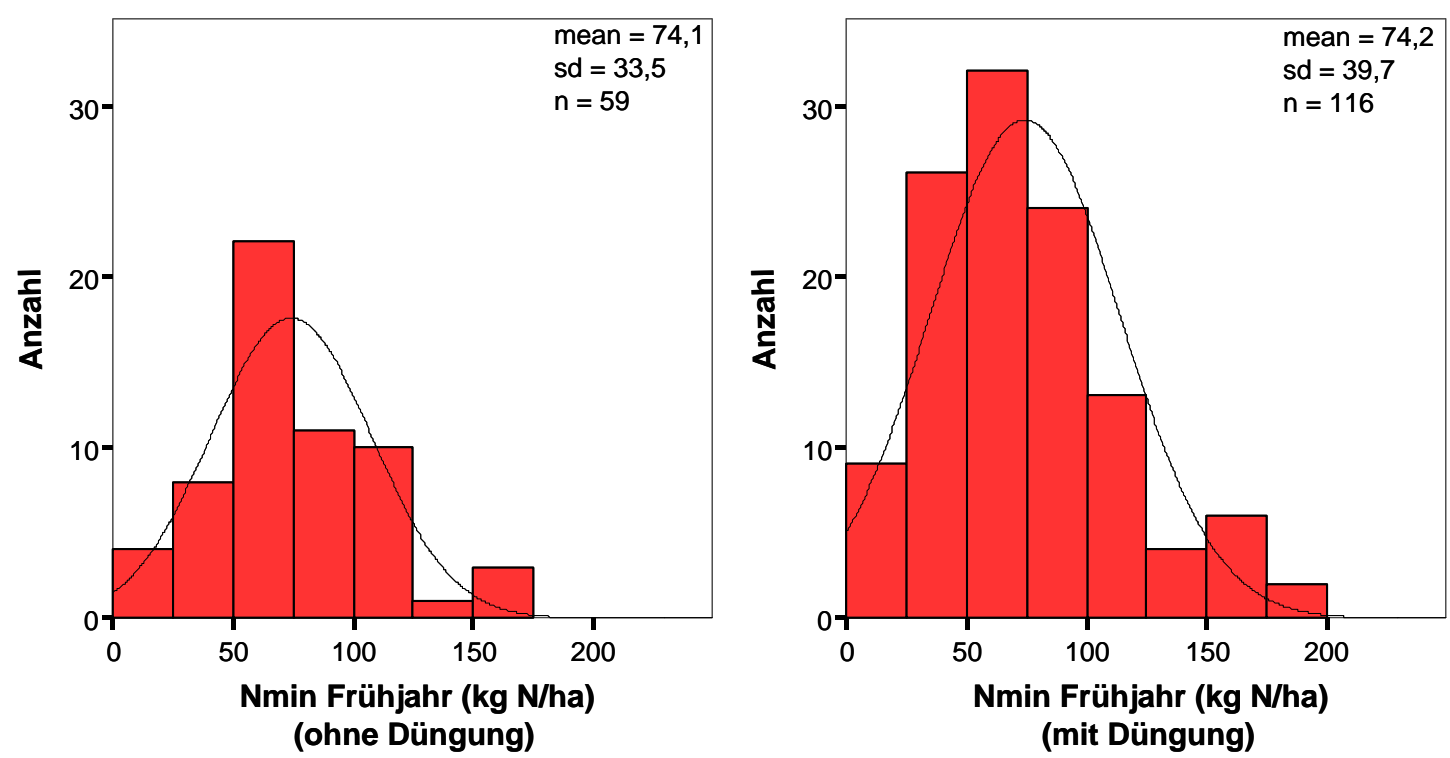

Abb. 43: Häufigkeitsverteilung der Frühjahrs $\mathrm{N}_{\min }$-Gehalte (0-90cm / Datenkategorie 3)

Die auf den 135 gedüngten Flächen ausgebrachte mineralische und organische Düngermenge lag im Mittel bei $97 \mathrm{~kg} \mathrm{~N} / \mathrm{ha}$ (Abb. 44). Auf 22 der gedüngten Flächen wurden organische Düngemittel alleine oder als Ergänzung zur mineralischen Düngung ausgebracht. Die Ausbringmenge variierte von 33 bis $131 \mathrm{~kg} \mathrm{~N} / \mathrm{ha}$. Die organisch gedüngten Flächen wurden mittels Gülle und 3 Flächen zusätzlich mit Festmist gedüngt. Auf 110 Flächen fand eine rein mineralische Düngung statt. Drei Flächen wurden auf Grund der hohen Frühjahrs $\mathrm{N}_{\min }$-Gehalte nicht mit Stickstoff gedüngt. 33 der gedüngten Flächen erhielten keine Unterfußdüngung. Die 102 verbleibenden Flächen wurden mit bis zu $45 \mathrm{~kg} \mathrm{~N} / \mathrm{ha}$ Unterfußdünger versorgt. Als Unterfußdünger wurde hauptsächlich Diamonphosphat eingesetzt.

Auf 17 der 78 ungedüngten Flächen wurde kein Unterfußdünger ausgebracht. Die weiteren 61 Flächen erhielten größtenteils 1 bis 2 dt/ha Diamonphosphat. 


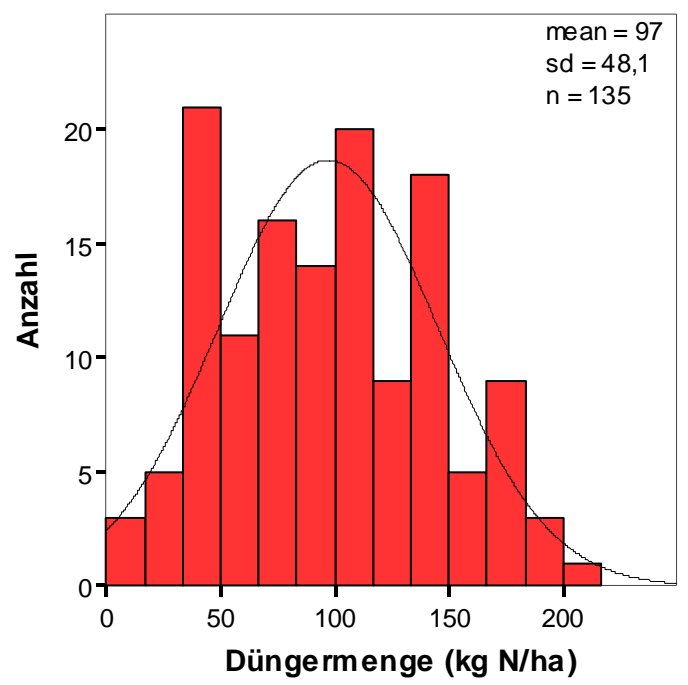

Abb. 44: Häufigkeitsverteilung der aufgewendeten Düngermenge (mineralisch und organisch) in $\mathrm{kg} / \mathrm{ha}$ auf den gedüngten Flächen (Datenkategorie 3)

Die Erträge der Silo- und Körnermaisflächen werden im Anhang (Anhang 16 und Anhang 17) dargestellt. Insgesamt wurde von 39 nicht gedüngten und 89 gedüngten Flächen der Silomaisertrag ermittelt. Auf den ungedüngten Flächen wurde im Mittel $139 \mathrm{dt} / \mathrm{ha}$ TM und auf den gedüngten $154 \mathrm{dt} / \mathrm{ha}$ TM geerntet. Die Körnermaiserträge unterscheiden sich zwischen den nicht gedüngten und den gedüngten Flächen im Mittel um ungefähr $10 \mathrm{dt} / \mathrm{ha}$. Auf den 38 ungedüngten Flächen wurde im Mittel 84 $\mathrm{dt} / \mathrm{ha}$ TM und auf den 37 gedüngten Flächen $94 \mathrm{dt} / \mathrm{ha}$ TM geerntet.

Von 77 ungedüngten und 126 gedüngten Flächen wurden N-Salden berechnet (Abb. 45). Die berechneten $\mathrm{N}$-Salden waren bei den nicht gedüngten Flächen mit $-130 \mathrm{~kg}$ $\mathrm{N} / \mathrm{ha}$ um $46 \mathrm{~kg} \mathrm{~N} / \mathrm{ha}$ niedriger als bei den gedüngten Flächen. 

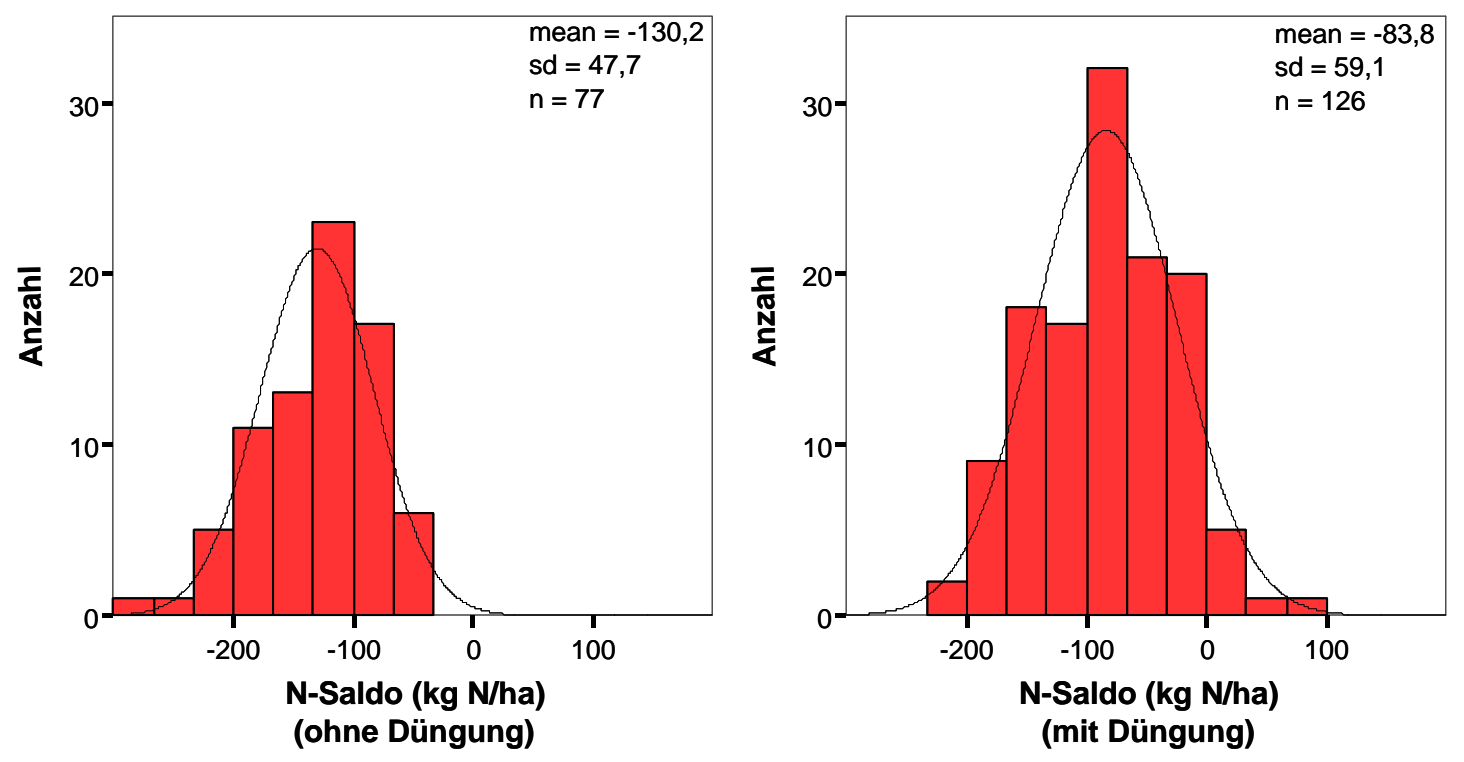

Abb. 45: Häufigkeitsverteilung der $\mathrm{N}$-Salden bei den Flächen mit und ohne Düngung (Datenkategorie 3)

Die Vorfrüchte der Maisflächen waren von 76 ungedüngten und 117 gedüngten Flächen bekannt (Anhang 18). Auf $61 \%$ der ungedüngten und auf $56 \%$ der gedüngten Flächen standen die Vorfrüchte Winterweizen, Wintergerste oder Silomais vor dem Maisanbau.

Angaben zum Zwischenfruchtanbau vor dem Mais lagen von 20 ungedüngten und 62 gedüngten Flächen vor (Anhang 19). 65\% der ungedüngten und 53\% der gedüngten Flächen lagen vor dem Anbau von Mais brach. Raps wurde auf 20\% der ungedüngten Flächen und Senf auf 19\% der gedüngten Flächen als Zwischenfrucht angebaut.

\section{Standortbezogene Daten und deren Struktur}

Die dominierende Bodenart in den Daten dieser Datenkategorie ist der Sandboden. Die Bodenarten sind von 78 ungedüngten und 121 gedüngten Flächen bekannt (Abb. 46). Zur Vergleichbarkeit mit den anderen Datenkategorien ist auch hier eine Gruppierung der Bodenarten vorgenommen worden (Anhang 6). 


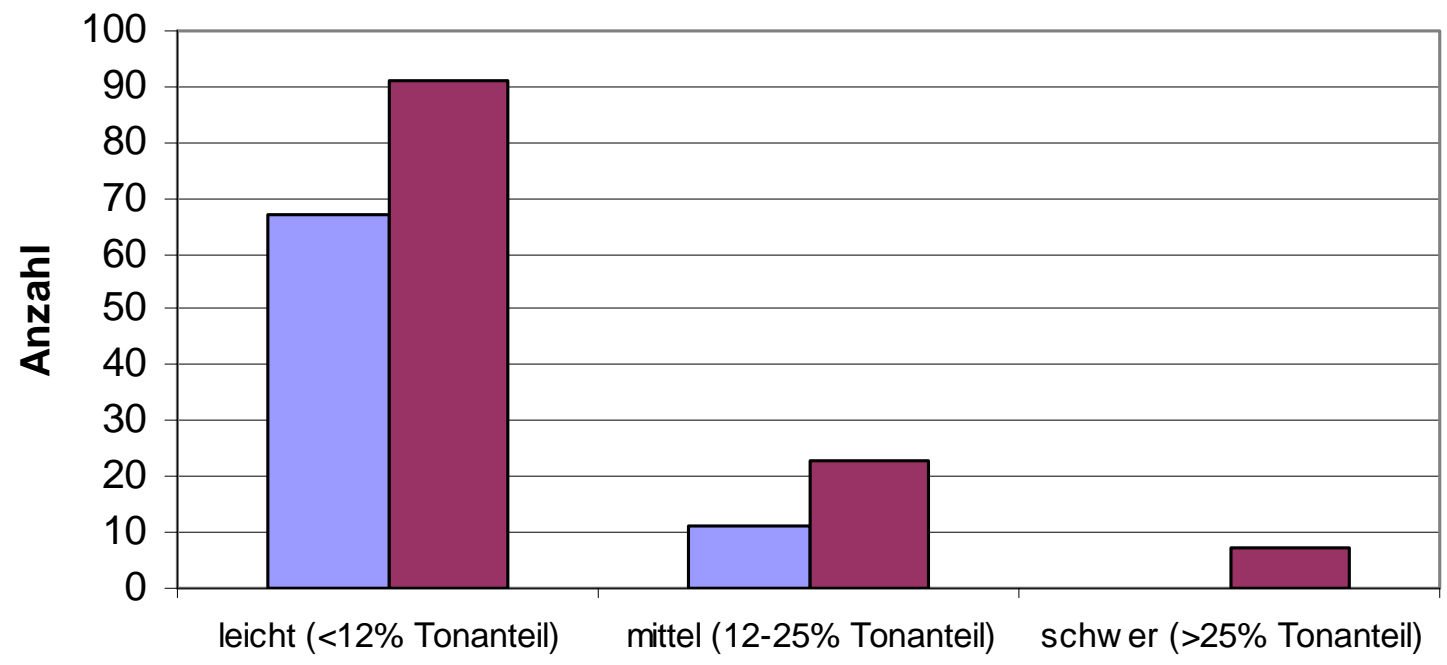

$\square$ Ohne Düngung $\square$ Mit Düngung

Abb. 46: Häufigkeitsverteilung der Flächen nach gruppierter Bodenart (Datenkategorie 3)

Als weiteres Bodenmerkmal liegt das C/N-Verhältnis von 68 Flächen vor. Das C/NVerhältnis ist bei den ungedüngten Flächen mit 18,4 im Mittel ein wenig weiter als auf den gedüngten Flächen mit 15,9.
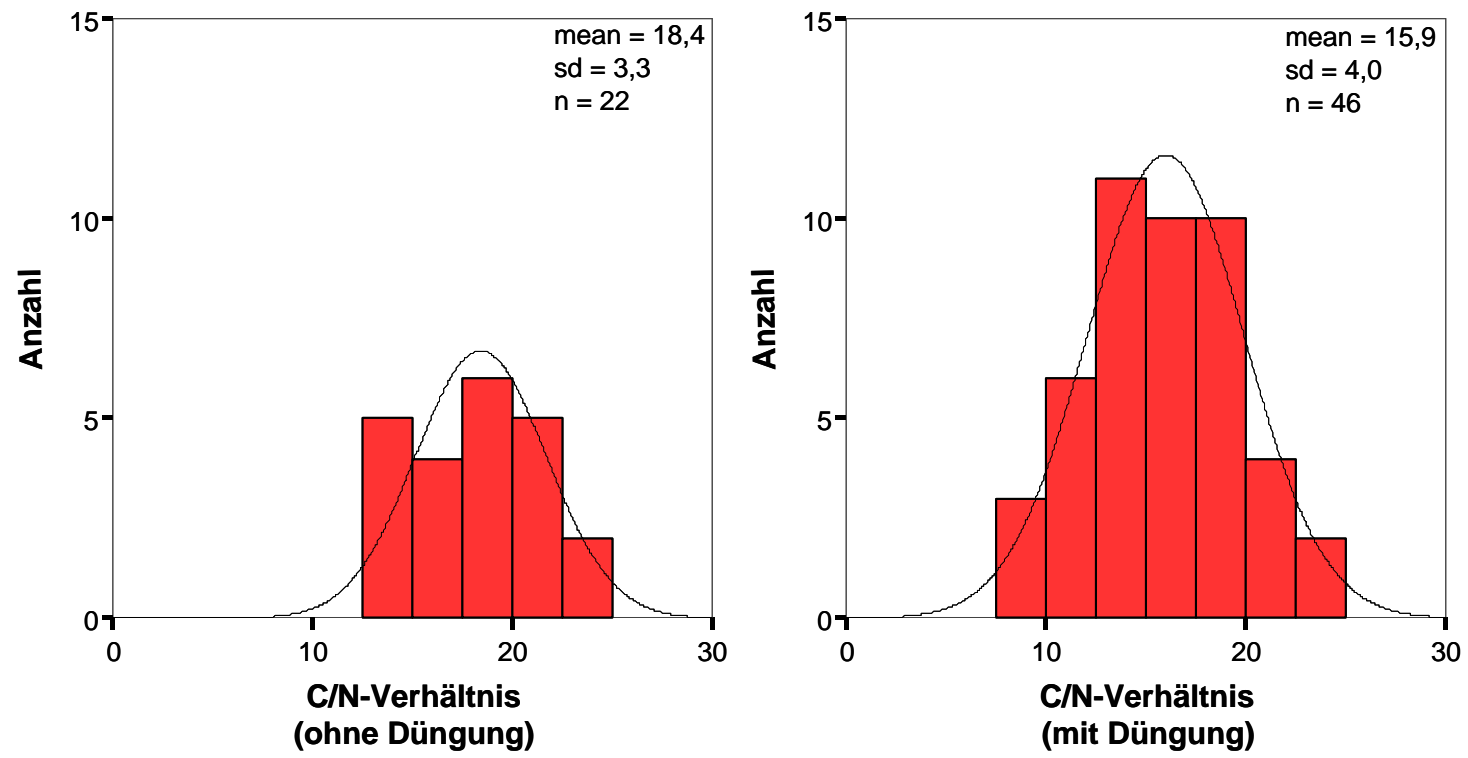

Abb. 47: Häufigkeitsverteilung der C/N-Verhältnisse je nach Düngung (Datenkategorie 3)

Ein weiteres Boden beschreibendes Merkmal ist der pH-Wert. Dieser ist von 66 ungedüngten und 91 gedüngten Flächen bekannt. Der Mittelwert liegt auf den 
ungedüngten Flächen bei 5,7 ( $\mathrm{sd}=0,6)$ und auf den gedüngten Flächen bei 5,8 ( $\mathrm{sd}=$ 0,7). Angaben zum $\mathrm{P}_{2} \mathrm{O}_{5}$-Gehalt und $\mathrm{K}_{2} \mathrm{O}$-Gehalt liegen von 53 ungedüngten und 76 gedüngten Flächen vor. Im Mittel der ungedüngten Flächen liegt der $\mathrm{P}_{2} \mathrm{O}_{5}$-Gehalt bei $30 \mathrm{mg}(\mathrm{sd}=11,4)$ und der gedüngten Flächen bei $28 \mathrm{mg}(\mathrm{sd}=9,4)$ je $100 \mathrm{~g}$ Boden. Der $\mathrm{K}_{2} \mathrm{O}-$ Gehalt der ungedüngten Flächen liegt im Mittel bei $14,6 \mathrm{mg}(\mathrm{sd}=6)$ und der gedüngten Flächen bei $15,1 \mathrm{mg}(\mathrm{sd}=12,9)$ je $100 \mathrm{~g}$ Boden.

\subsubsection{Analytische Statistik}

Die ungedüngten und gedüngten Parzellen der Exaktversuche werden zunächst mittels der Faktorenanalyse untersucht. Die in die Faktorenanalyse eingeflossenen Variablen sind anhand des KMO-Maßes und des MSA-Wertes ausgewählt worden. In der Tab. 18 und Tab. 19 sind die Ergebnisse der Faktorenanalyse von den ungedüngten Parzellen dargestellt. Durch die eine gebildete Komponente (Faktor) werden $60 \%$ der Varianz beschrieben.

Tab. 18: Eigenwert und erklärte Varianz der Faktorenanalyse (ohne Düngung I Datenkategorie 3)

\begin{tabular}{lc}
\hline & Komponente 1 \\
\hline Eigenwert & 3,01 \\
\% der Varianz & 60,11 \\
Kumulierte \% & 60,11 \\
\hline
\end{tabular}

Welche Variablen in die Komponente eingeflossen sind, zeigt die mit Hilfe der Hauptkomponentenanalyse ermittelte Komponentenmatrix (Tab. 19). Da in diesem Fall keine zwei Komponenten ausgewiesen sind, kann keine Rotation durchgeführt werden. Die Faktorladung entspricht der Korrelation zwischen einer Variablen und einem Faktor. Je höher diese Ladung ist, desto besser misst diese Variable (Item) den Faktor (relevante $=$ fett gedruckt). Die Komponente 1 besteht aus den Variablen $\mathrm{N}_{\min }$-Frühjahr, N-Saldo, Ackerzahl, Gesamt-N Gehalt und dem $\mathrm{C}_{\mathrm{t}}$-Gehalt.

Tab. 19: Komponentenmatrix mit der entsprechenden Faktorladung (ohne Düngung $I$ Datenkategorie 3)

\begin{tabular}{lc}
\hline Variablen & Komponente 1 \\
\hline $\mathrm{N}_{\min }$ Frühjahr (0-90cm) & 0,851 \\
Gesamt-N (Boden) & 0,927 \\
$\mathrm{C}_{\mathrm{t}}$ (Boden) & 0,654 \\
$\mathrm{~N}$-Saldo & $-0,694$ \\
Ackerzahl & 0,717 \\
\hline
\end{tabular}


Die hier ermittelte Komponente dient als Variable in der weiteren Untersuchung. So sind mittels der Regression Faktorwerte geschätzt worden. Der Einfluss der Faktorwerte auf den Herbst $\mathrm{N}_{\min }$-Gehalt wird dann mittels der Korrelation nach Pearson untersucht (Abb. 48). Die Komponente 1 zeigt einen deutlichen signifikanten Zusammenhang zum Herbst $\mathrm{N}_{\min }$-Gehalt auf.

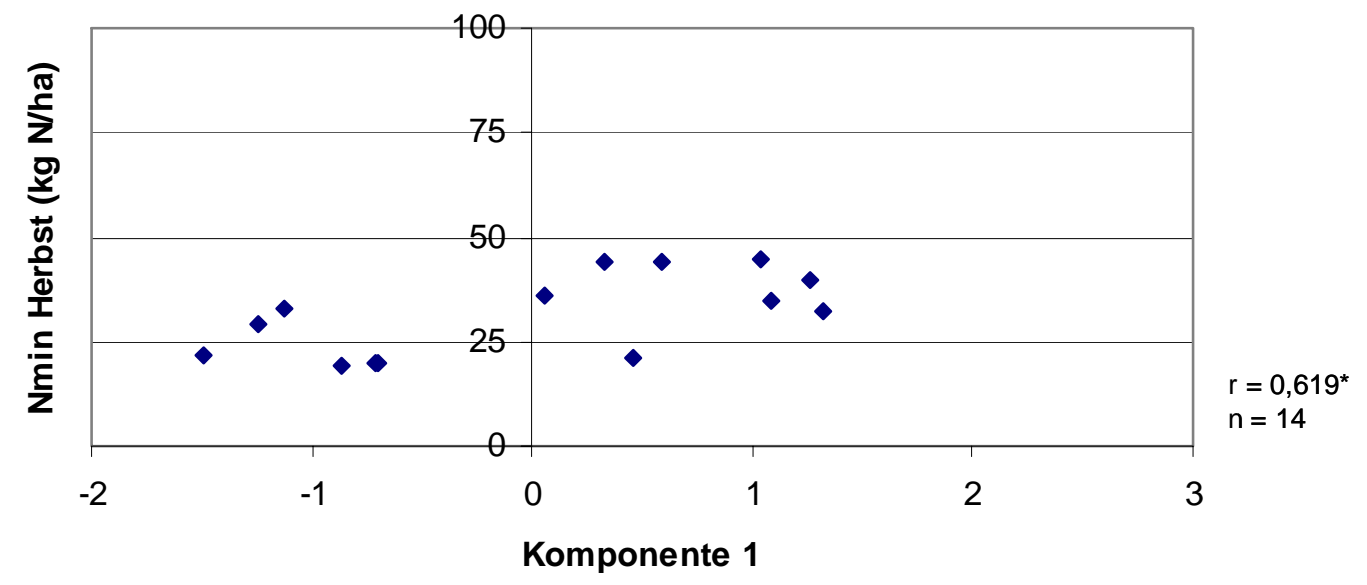

Abb. 48: Beziehung zwischen dem Herbst $\mathrm{N}_{\min }$-Gehalt und der Komponente 1 der Faktorenanalyse (ohne Düngung / Korrelation nach Pearson I Datenkategorie 3)

Das Ergebnis der Faktorenanalyse der gedüngten Parzellen in Tab. 20 zeigt, dass die ersten zwei Komponenten (Faktoren) 86\% der Varianz beschreiben.

Tab. 20: Eigenwerte und erklärte Varianzen der Faktorenanalyse (mit Düngung / Datenkategorie 3)

\begin{tabular}{lcc}
\hline & Komponente 1 & Komponente 2 \\
\hline Eigenwert & 2,62 & 1,70 \\
\% der Varianz & 52,31 & 33,86 \\
Kumulierte \% & 52,31 & 86,16 \\
\hline
\end{tabular}

Inwieweit die einzelnen Variablen in die Komponenten eingeflossen sind, zeigt die rotierte Komponentenmatrix (Tab. 21). Die Komponente 1 wird im Wesentlichen durch die Variablen $\mathrm{N}_{\min }$-Frühjahr, $\mathrm{N}$-Saldo und Düngung erklärt. In der Komponente 2 sind es der Gesamt-N Gehalt und der $\mathrm{C}_{\mathrm{t}}$-Gehalt des Bodens.

Tab. 21: Rotierte Komponentenmatrix mit der entsprechenden Faktorladung (mit Düngung I Datenkategorie 3)

\begin{tabular}{|c|c|c|}
\hline \multirow[t]{2}{*}{ Variablen } & \multicolumn{2}{|c|}{ Komponente } \\
\hline & 1 & 2 \\
\hline$N_{\min }$ Frühjahr $(0-90 \mathrm{~cm})$ & $-0,832$ & 0,308 \\
\hline Gesamt-N (Boden) & $-0,491$ & 0,805 \\
\hline$C_{t}$ (Boden) & 0,004 & 0,965 \\
\hline N-Saldo & 0,928 & $-0,058$ \\
\hline Düngung (min. + org.) & 0,906 & $-0,128$ \\
\hline
\end{tabular}


Die hier ermittelten Komponenten dienen als Variablen in der weiteren Untersuchung. So sind mittels der Regression Faktorwerte geschätzt worden. Der Einfluss der Faktorwerte auf den Herbst $\mathrm{N}_{\min }$-Gehalt wird dann mittels der Korrelation nach Pearson untersucht (Abb. 49). Die Korrelation nach Pearson weist die nahezu gleichen Ergebnisse wie eine partielle Korrelation auf, daher wird die Korrelation nach Pearson beibehalten. Die Komponente 2 zeigt einen signifikanten Zusammenhang zum Herbst $\mathrm{N}_{\min }$-Gehalt auf. Die Variablen Gesamt- $\mathrm{N}$ und $\mathrm{C}_{\mathrm{t}}$-Gehalt scheinen den größten Einfluss auf den Herbst $\mathrm{N}_{\min }$-Gehalt auszuüben.

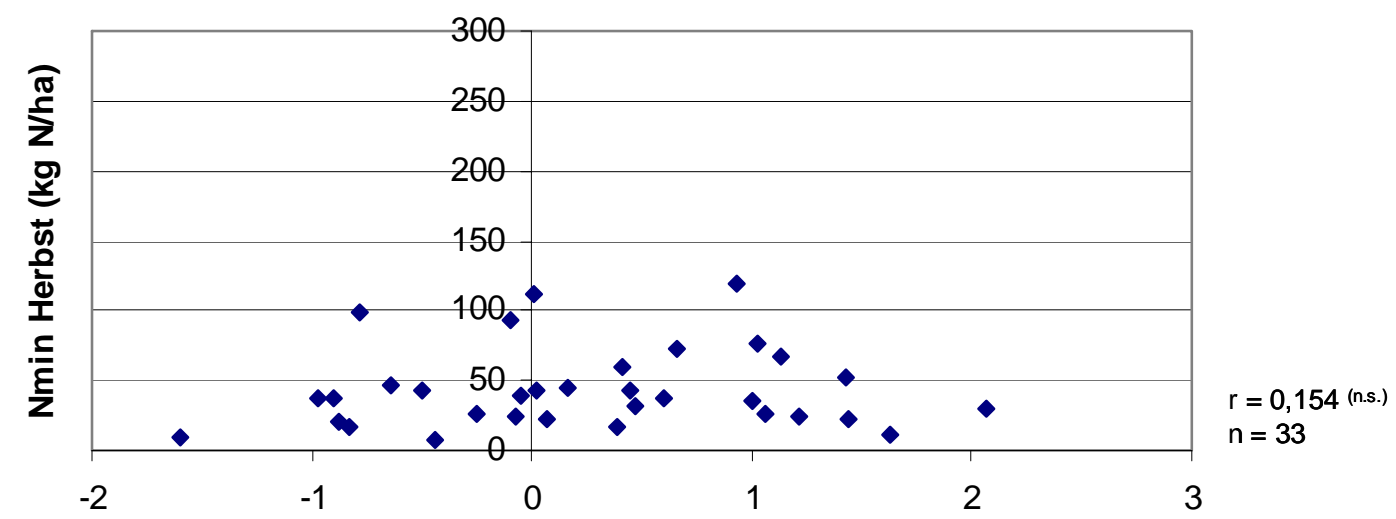

Komponente 1

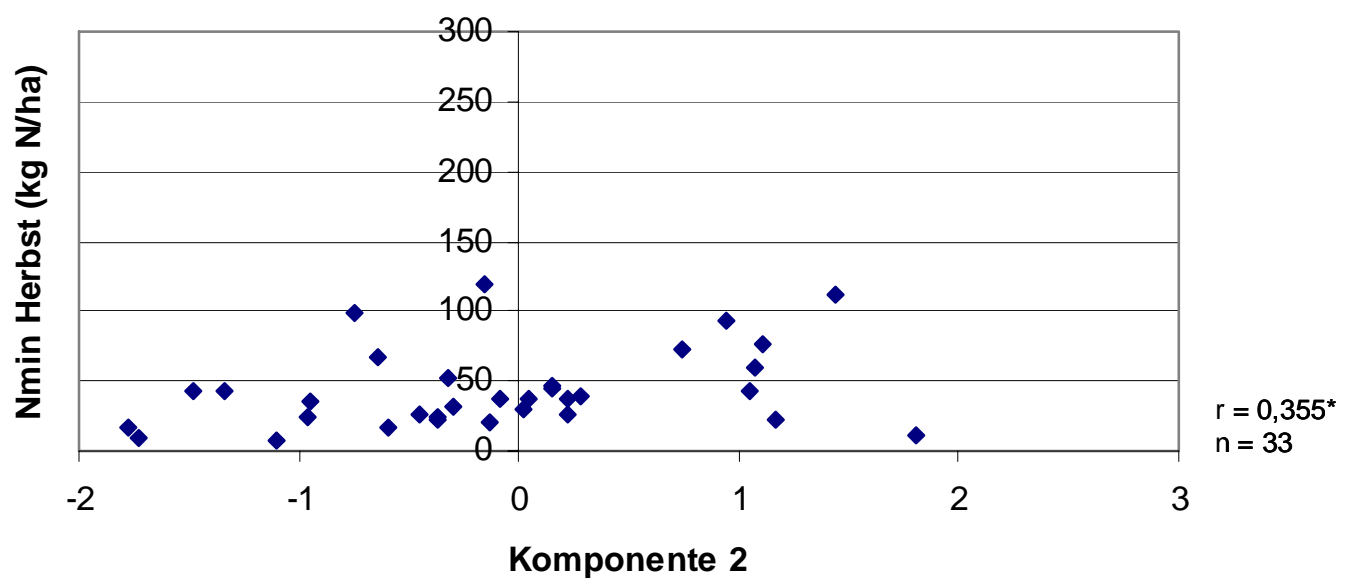

Abb. 49: Beziehung zwischen dem Herbst $\mathrm{N}_{\min }$-Gehalt und den einzelnen Komponenten der Faktorenanalyse (mit Düngung / Korrelation nach Pearson / Datenkategorie 3)

Nach der Analyse der Struktur der Daten mittels der Faktorenanalyse werden die Daten in den nachfolgenden Diagrammen ausgewählten Variablen gegenübergestellt. Das in Abb. 50 dargestellte Ergebnis zeigt, dass sowohl die ausgebrachte Düngermenge als auch der Herbst $\mathrm{N}_{\min }$-Gehalt auf den gedüngten 
Flächen stark streut, und dass es keinen Zusammenhang zwischen diesen beiden Merkmalen gibt.

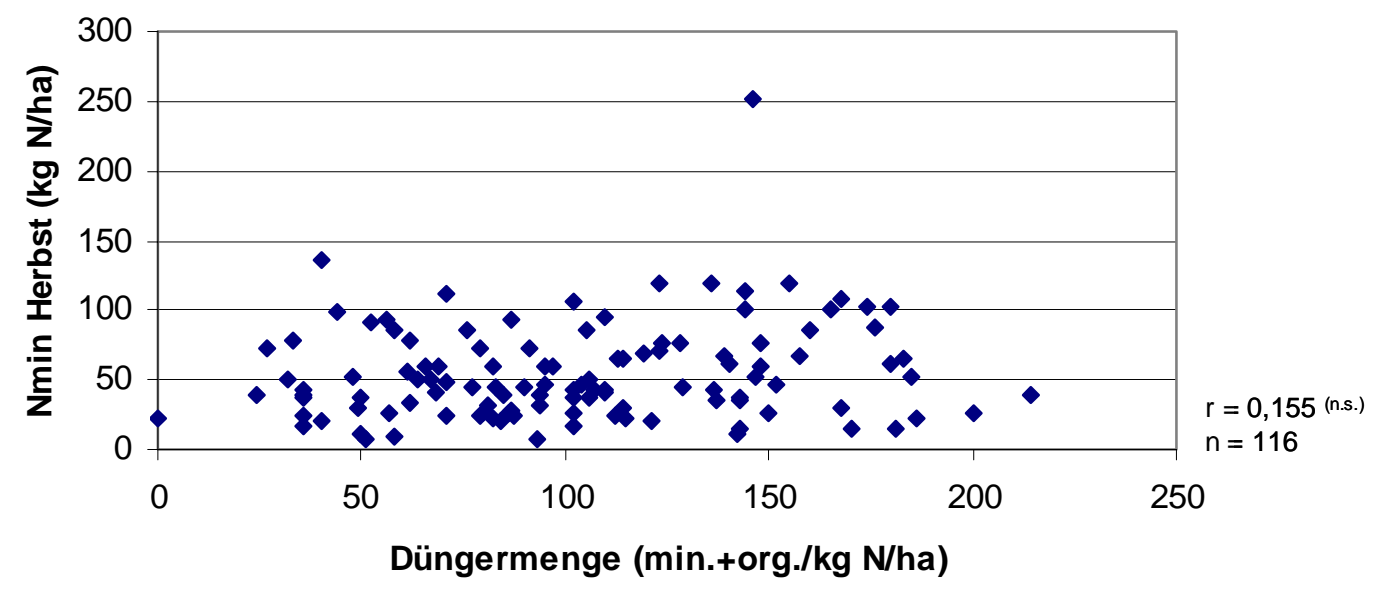

Abb. 50: Herbst $\mathrm{N}_{\min }$-Gehalt im Verhältnis zur ausgebrachten mineralischen und organischen Düngermenge (Korrelation nach Pearson / Datenkategorie 3)

Eine weitere Differenzierung der Abb. 50 nach der Nutzungsart führt nicht zu signifikanten Zusammenhängen. Zur Prüfung des Einflusses der Bodenart auf den Zusammenhang zwischen der Düngermenge und dem Herbst $\mathrm{N}_{\text {min }}$-Gehalt sind in der Tab. 22 Korrelationen nach Pearson berechnet worden. Die Korrelationskoeffizienten zeigen keine signifikanten Zusammenhänge auf.

Tab. 22: Korrelation (Pearson) zwischen der N-Düngermenge (mineralisch/organisch) und dem Herbst $\mathrm{N}_{\text {min }}$-Gehalt (0-90cm / Datenkategorie 3)

\begin{tabular}{lcc}
\hline Bodenart (gruppiert) & Korrelationskoeffizient (r) & Anzahl (n) \\
\hline leicht & $0,076^{(\text {n.s. }}$ & 81 \\
mittel & $0,341^{(\text {n.s. }}$ & 18 \\
schwer & $-0,478^{(\text {n.s. }}$ & 4 \\
leicht / mittel / schwer & $0,123^{(\text {n.s. }}$ & 103 \\
(zusammen) & & \\
\hline
\end{tabular}

Unterschiede in der applizierten Düngermenge zwischen den Bodenartgruppen können nicht festgestellt werden. Der Zusammenhang zwischen dem Herbst $\mathrm{N}_{\text {min- }}$ Gehalt und dem Anteil der ausgebrachten mineralischen Düngung an der gesamten Düngermenge wird in Abb. 51 verdeutlicht. Das Streudiagramm zeigt keinen Zusammenhang auf. Der Mittelwert der mineralischen Düngung liegt in Abb. 51 bei $91 \mathrm{~kg} \mathrm{~N} / \mathrm{ha}$. 


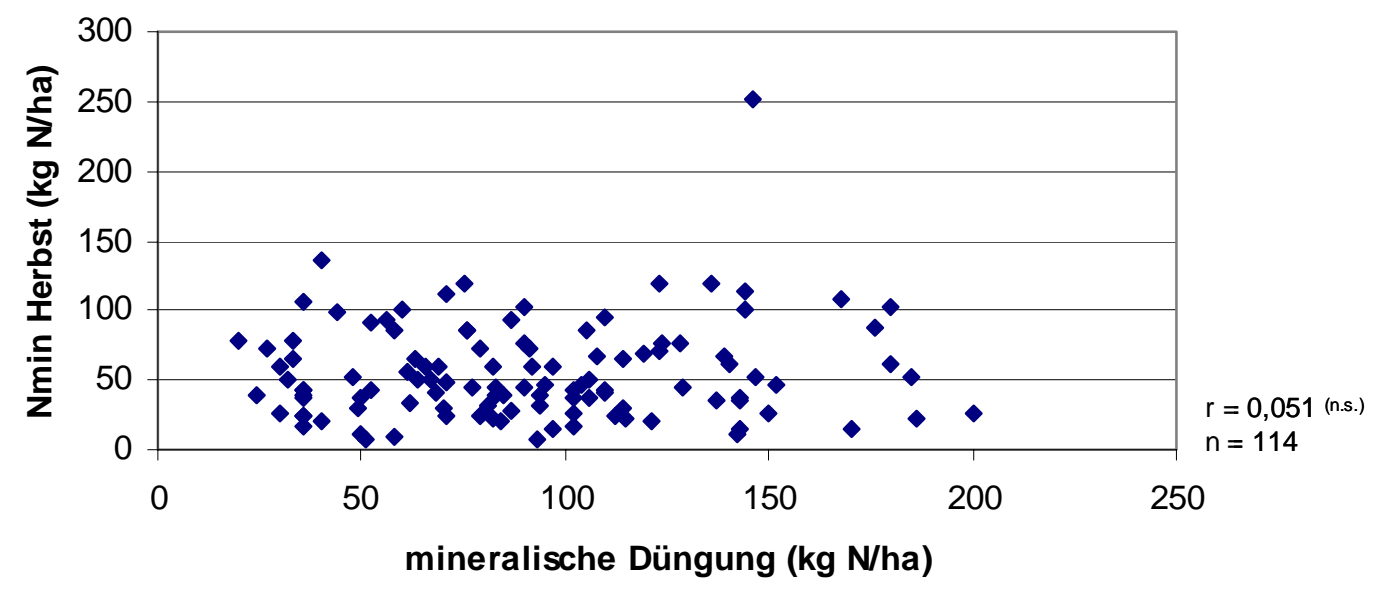

Abb. 51: Herbst $\mathrm{N}_{\min }$-Gehalte im Verhältnis zur ausgebrachten mineralischen Düngermenge (Korrelation nach Pearson I Datenkategorie 3)

In der Abb. 52 wird der Zusammenhang zwischen der organischen Düngermenge und der Herbst $\mathrm{N}_{\min }$-Menge dargestellt. Ein signifikanter Zusammenhang besteht nicht. Im Mittel wurden $76 \mathrm{~kg} \mathrm{~N} / \mathrm{ha}$ an organischer Düngung ausgebracht (Abb. 52). Auf 96 Flächen wurde keine organische Düngung appliziert.

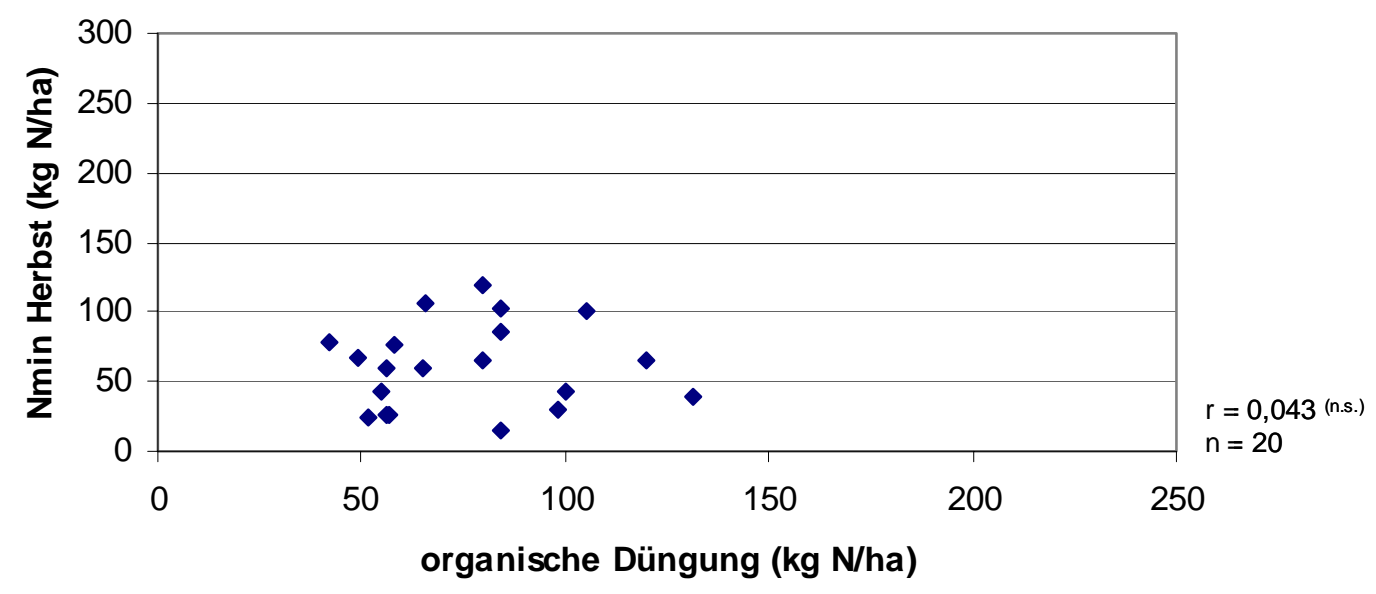

Abb. 52: Herbst $\mathrm{N}_{\min }$-Gehalte im Verhältnis zur ausgebrachten organischen Düngermenge (Korrelation nach Pearson I Datenkategorie 3)

Auf den Flächen, die nur mineralisch (110 Flächen) gedüngt wurden, zeigen sich ebenfalls keine signifikanten Zusammenhänge zum Herbst $\mathrm{N}_{\text {min }}$-Gehalt. Eine Fläche wurde rein organisch gedüngt. 21 Flächen wurden kombiniert gedüngt und auf 3 Flächen wurde keine Düngung appliziert (Anhang 20).

Zwischen dem $\mathrm{N}$-Saldo und der Herbst $\mathrm{N}_{\text {min }}$-Menge besteht auf den ungedüngten Flächen kein Zusammenhang (Abb. 53). Die gedüngten Flächen weisen eine 
schwache Korrelation auf. Der N-Saldo beträgt auf den ungedüngten Flächen im Mittel -132 kg N/ha und auf den gedüngten Flächen $-80 \mathrm{~kg} \mathrm{~N} / \mathrm{ha}$, die korrespondierenden Herbst $\mathrm{N}_{\min }$-Gehalte liegen bei den nicht gedüngten Flächen bei $34 \mathrm{~kg} \mathrm{~N} / \mathrm{ha}$ und auf den gedüngten Flächen bei $56 \mathrm{~kg} \mathrm{~N} / \mathrm{ha}$. Eine Differenzierung nach Bodenartgruppen (leicht - schwer) führt nicht zu höheren und signifikanten Korrelationskoeffizienten.

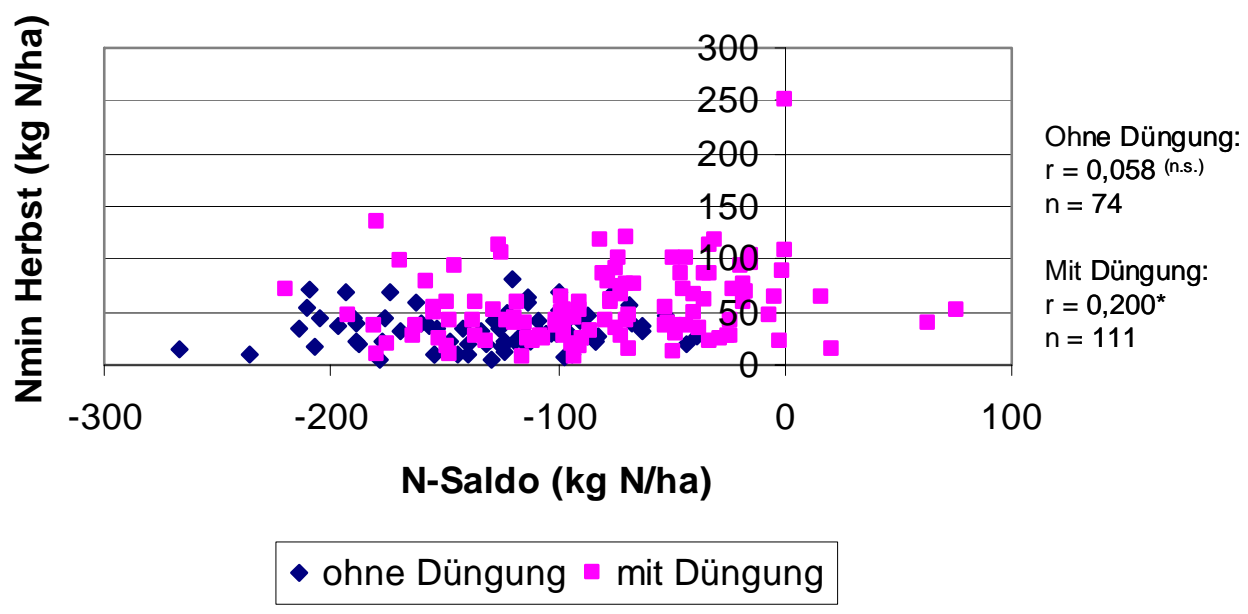

Abb. 53: Herbst $\mathrm{N}_{\min }$-Gehalte versus $\mathrm{N}-$ Saldo bei den nicht gedüngten und gedüngten Flächen (Korrelation nach Pearson I Datenkategorie 3)

Der Einfluss der Bodenartgruppen auf den Herbst $\mathrm{N}_{\text {min }}$-Gehalt ist mittels des KruskalWallis Tests untersucht worden. Es kann weder bei den Flächen mit noch ohne Düngung ein Einfluss der Bodenartgruppe festgestellt werden. Von den ungedüngten Flächen liegt keine in der Bodenartgruppe „schwer“ (Abb. 54). 

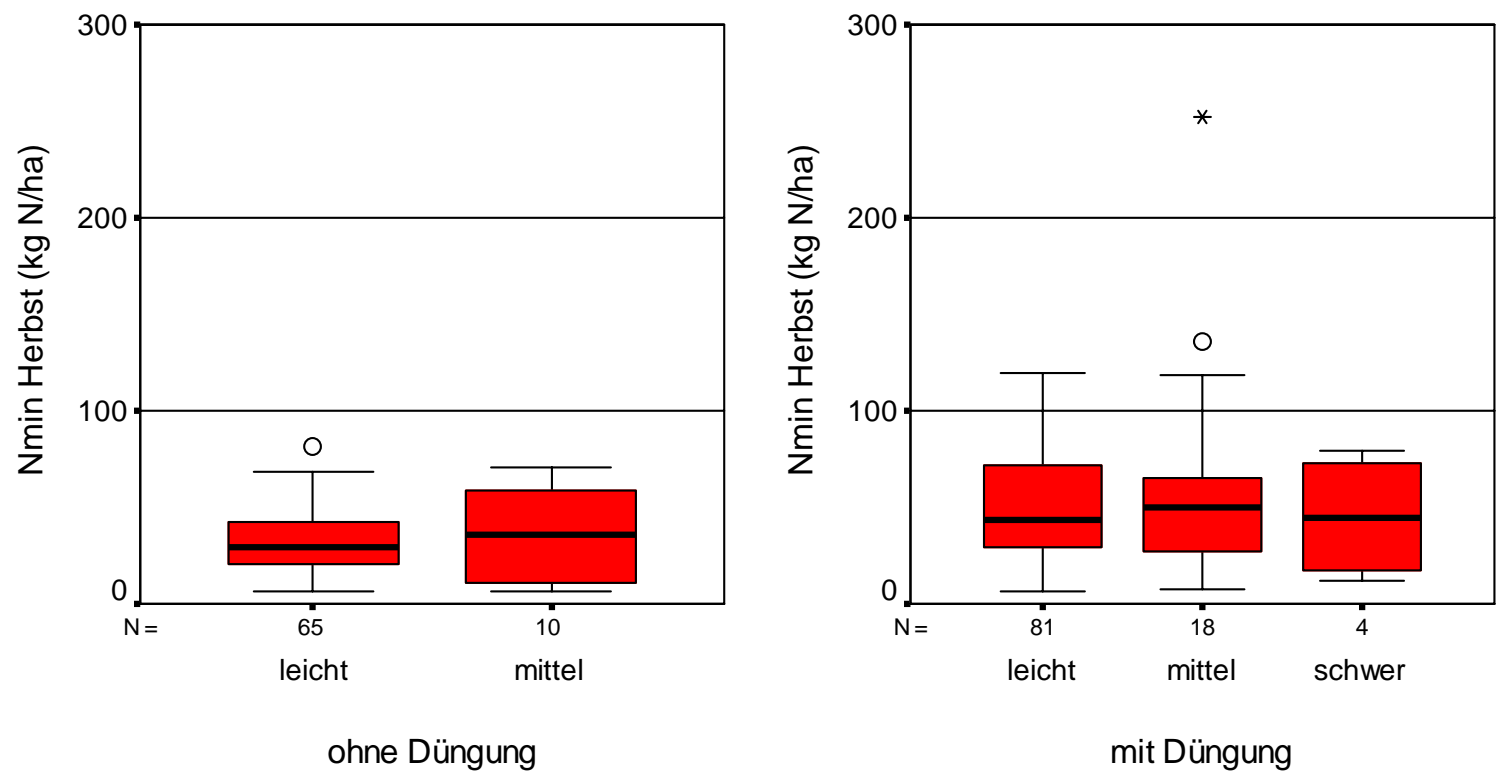

Abb. 54: Herbst $\mathrm{N}_{\min }-$ Mengen je nach Bodenartgruppe (Datenkategorie 3)

Aus der Abb. 55 geht hervor, dass keine Beziehung zwischen der Herbst $\mathrm{N}_{\min }$-Menge und dem C/N-Verhältnis besteht. Das C/N-Verhältnis ist im Mittel auf den 22 ungedüngten Flächen mit 18,4 ein wenig weiter als auf den 38 gedüngten Flächen mit 16,7 .

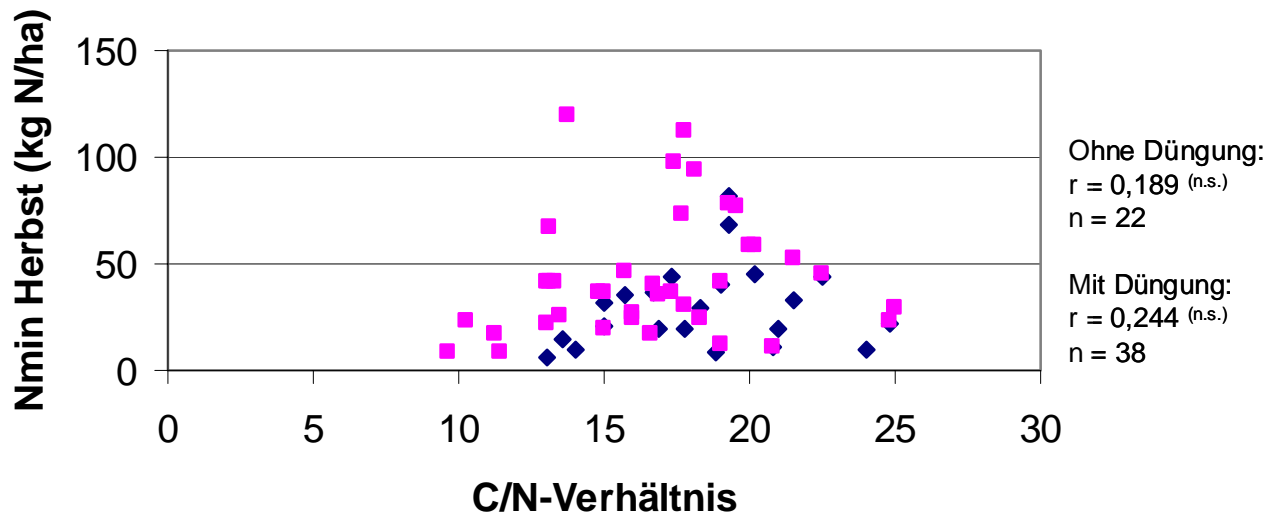

- ohne Düngung - mit Düngung

Abb. 55: Herbst $\mathrm{N}_{\text {min }}$-Gehalte in Abhängigkeit vom $\mathrm{C} / \mathrm{N}$-Verhältnis des Bodens (Korrelation nach Pearson I Datenkategorie 3)

Der Kruskal-Wallis Test bestätigt bei den gedüngten und ungedüngten Flächen einen signifikanten Unterschied $(p<0,01)$ zwischen den Bodenartgruppen und dem $\mathrm{C} / \mathrm{N}$ Verhältnis. Die Bodenartgruppen mit mehr als $12 \%$ Tonanteil weisen engere $\mathrm{C} / \mathrm{N}$ Verhältnisse auf als die leichte Bodenartgruppe mit weniger als 12\% Tonanteil. 
Eine enge Korrelation $(r=0,741)$ besteht auf den ungedüngten Flächen zwischen dem Gesamt-N Gehalt des Bodens und der Herbst $\mathrm{N}_{\min }$-Menge (Abb. 56). Die Korrelation ist auf einem Niveau von $<0,001$ signifikant. Auf den gedüngten Flächen ist auf Grund der starken Streuung keine Beziehung nachzuweisen. Eine Differenzierung nach Bodenartgruppen ist nicht möglich, da nur wenige Werte für die Bodenartgruppen mit mehr als $12 \%$ Tonanteil zur Verfügung stehen.

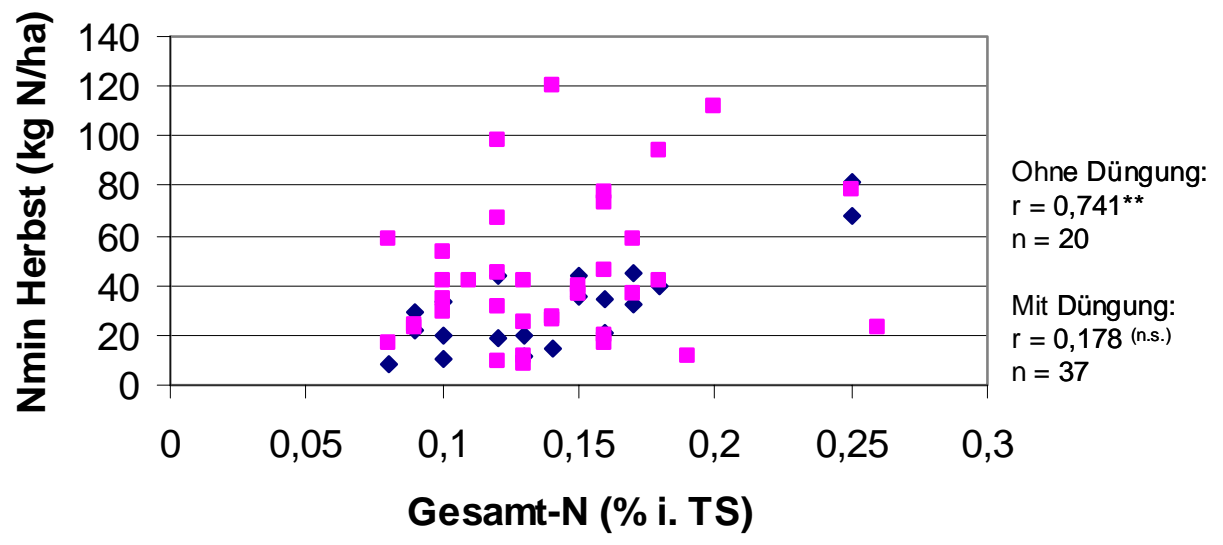

- ohne Düngung - mit Düngung

Abb. 56: Herbst $\mathrm{N}_{\min }$-Gehalte in Abhängigkeit vom Gesamt-N Gehalt des Bodens (Korrelation nach Pearson I Datenkategorie 3)

Der Zusammenhang zwischen dem $\mathrm{C}_{\mathrm{t}}$-Gehalt des Bodens und dem Herbst $\mathrm{N}_{\min }{ }^{-}$ Gehalt ist auf den 22 ungedüngten Flächen mit einem Korrelationskoeffizienten von 0,808 auf einem Niveau von $<0,001$ signifikant. Die gedüngten Flächen weisen mit Zunahme des $\mathrm{C}_{\mathrm{t}}$-Gehaltes eine stärkere Streuung auf. Deshalb ist der Korrelationskoeffizient nach Pearson mit 0,366 und einem Signifikanzniveau von 0,024 niedriger. Eine Differenzierung nach Bodenartgruppen ist auf Grund der wenigen vorliegenden Werte für die Bodenartgruppen mit mehr als $12 \%$ Tonanteil nicht möglich. 


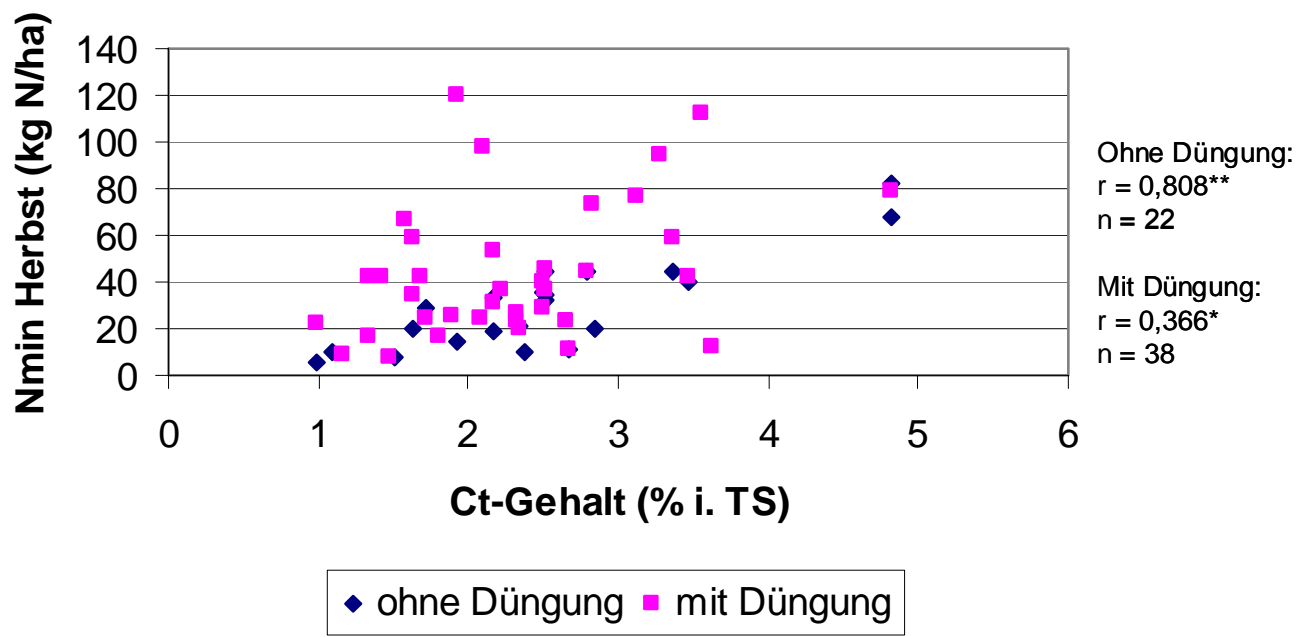

Abb. 57: Herbst $N_{\min }$-Gehalte in Abhängigkeit vom $C_{t}$-Gehalt des Bodens (Korrelation nach Pearson I Datenkategorie 3)

Die Beziehung zwischen dem Frühjahrs $\mathrm{N}_{\min }$-Gehalt und dem folgenden Herbst $\mathrm{N}_{\text {min }^{-}}$ Gehalt zeigt die Tab. 23. Die Korrelationen wurden in Abhängigkeit von den drei gebildeten Bodenartgruppen ermittelt. Wie aus der Tabelle hervorgeht, besteht zwischen den Frühjahrs und Herbst $\mathrm{N}_{\min }$-Gehalten auf den ungedüngten Flächen in der leichten Bodenartgruppe eine signifikante Korrelation. Die gleichen Flächen finden sich bei der Betrachtung aller Bodenartgruppen wieder und zeigen dementsprechend den gleichen Zusammenhang auf. Auf zwei gedüngten Flächen ist in der schweren Bodenartgruppe ein signifikanter Zusammenhang zu finden.

Tab. 23: Korrelation (Pearson) zwischen dem Frühjahrs und dem nachfolgendem Herbst $\mathbf{N}_{\text {min }}{ }^{-}$ Gehalt (0-90cm / Datenkategorie 3)

\begin{tabular}{|c|c|c|c|}
\hline Bodengruppe & $\mathbf{N}_{\min }$ Termin & $\begin{array}{c}\mathbf{N}_{\min } \text { Herbst } \\
\text { (ohne Düngung) }\end{array}$ & $\begin{array}{c}\mathrm{N}_{\min } \text { Herbst } \\
\text { (mit Düngung) }\end{array}$ \\
\hline $\begin{array}{l}\text { Leicht }(<12 \% \\
\text { Tonanteil) }\end{array}$ & $\begin{array}{l}N_{\min } \text { Frühjahr } \\
\text { (vor 1. Mai) } \\
N_{\min } \text { Frühjahr } \\
\text { (ab 1. Mai) }\end{array}$ & $\begin{array}{c}, 033^{(n . s .)} \\
(n=18) \\
, 558^{\star *} \\
(n=26)\end{array}$ & $\begin{array}{c}, 367^{\text {(n.s.) }} \\
(\mathrm{n}=23) \\
, 191^{(\text {n.s. })} \\
(\mathrm{n}=38)\end{array}$ \\
\hline $\begin{array}{l}\text { Mittel (12-25\% } \\
\text { Tonanteil) }\end{array}$ & $\begin{array}{l}N_{\min } \text { Frühjahr } \\
\text { (vor 1. Mai) } \\
N_{\min } \text { Frühjahr } \\
\text { (ab 1. Mai) }\end{array}$ & $\begin{array}{c}-, 457^{(\text {n.s. })} \\
(n=4) \\
-\end{array}$ & $\begin{array}{c}-, 235^{\text {(n.s.) }} \\
(n=10) \\
, 878^{(\text {n.s. })} \\
(n=4)\end{array}$ \\
\hline $\begin{array}{l}\text { Schwer (>25\% } \\
\text { Tonanteil) }\end{array}$ & $\begin{array}{l}N_{\min } \text { Frühjahr } \\
\text { (vor 1. Mai) } \\
N_{\min } \text { Frühjahr } \\
\text { (ab 1. Mai) }\end{array}$ & - & $\begin{array}{c}1,00 * * \\
(n=2) \\
1,00 * * \\
(n=2)\end{array}$ \\
\hline Leicht, Mittel, Schwer & $\begin{array}{l}N_{\min } \text { Frühjahr } \\
\text { (vor 1. Mai) } \\
N_{\min } \text { Frühjahr } \\
\text { (ab 1. Mai) }\end{array}$ & $\begin{array}{c}-, 149^{(\text {n.s. })} \\
(n=22) \\
, 558 * * \\
(n=26)\end{array}$ & $\begin{array}{l}, 096^{\text {(n.s.) }} \\
(n=35) \\
, 232^{(\text {n.s. })} \\
(n=44) \\
\end{array}$ \\
\hline
\end{tabular}


In Abb. 58 ist der Einfluss der Ackerzahl auf die Herbst $\mathrm{N}_{\text {min }}$-Menge dargestellt. Eine Beziehung zwischen der Ackerzahl und den Herbst $\mathrm{N}_{\min }$-Mengen kann nicht festgestellt werden.

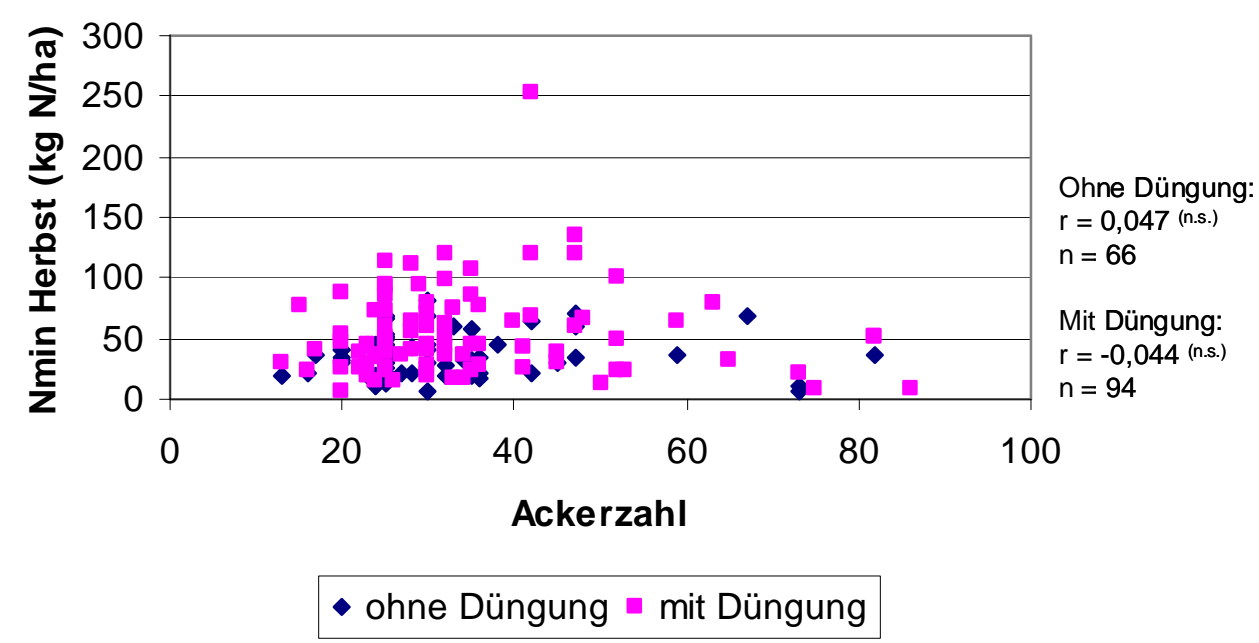

Abb. 58: Herbst $\mathrm{N}_{\min }$-Gehalte in Abhängigkeit von der Ackerzahl (Korrelation nach Pearson I Datenkategorie 3)

Eine Beziehung zwischen dem pH-Wert des Bodens und dem $\mathrm{N}_{\min }$-Gehalt im Herbst ist mittels der Korrelation nach Pearson nicht nachzuweisen. Die $\mathrm{P}_{2} \mathrm{O}_{5}$ Gehalte des Bodens zeigen ebenfalls keinen Einfluss auf den Herbst $\mathrm{N}_{\min }$-Gehalt.

Die bisher mit Korrelationen untersuchten Zusammenhänge zwischen der abhängigen Variable Herbst $\mathrm{N}_{\min }$-Gehalt und den unabhängigen Variablen werden nun mittels der multiplen Regression untersucht. Die multiple Regression wird deshalb eingesetzt, weil der Einfluss eines Faktors in den Korrelationen durch einen anderen verschleiert werden kann. Für die ungedüngten Flächen wird eine multiple Regression mit der abhängigen Herbst $\mathrm{N}_{\text {min- }}$-Gehalt und den erklärenden Variablen Gesamt-N Gehalt, $\mathrm{C}_{\mathrm{t}}$-Gehalt, Frühjahrs $\mathrm{N}_{\min }$-Gehalt und dem N-Saldo gerechnet. Diese Regression wird aber auf Grund der festgestellten Autokorrelation nicht abgebildet. Die multiple Regression der gedüngten Flächen mit den unabhängigen Variablen Gesamt-N Gehalt, $\mathrm{C}_{\mathrm{t}}$-Gehalt, Frühjahrs $\mathrm{N}_{\min }$-Gehalt und dem N-Saldo wird in Tab. 24 dargestellt.

Die schrittweise multiple Regression weist mit einem $r^{2}$-Wert von 0,151 eine Beziehung zwischen der abhängigen Variable und der unabhängigen Variable $\left(\mathrm{C}_{t^{-}}\right.$ Gehalt) nach. 
Tab. 24: Multiple Regression mit dem Herbst $\mathbf{N}_{\min }$-Gehalt als abhängige Variable (Regressor I Datenkategorie 3)

\begin{tabular}{lcccc}
\hline & $\mathbf{b}$ & $\mathbf{S F}$ & $\mathbf{t}$ & $\mathbf{p}$ \\
\hline $\begin{array}{l}\text { Konstante } \\
\begin{array}{l}\text { C-Gehalt } \\
\text { (Boden) }\end{array}\end{array}$ & 1,833 & 0,750 & 2,443 & 0,020 \\
& 1,157 & 0,494 & 2,344 & 0,026 \\
& & & & \\
\hline & $\mathbf{r}^{2}=\mathbf{0 , 1 5 1}$ & $\mathrm{F}=5,496$ & $\mathrm{p}=0,026$ & $\mathrm{n}=33$ \\
\hline
\end{tabular}

Einfluss der Vor- und Zwischenfrucht auf den Frühjahrs und Herbst $\mathbf{N}_{\text {min }}$-Gehalt Zur Aufdeckung der Wirkung des Zwischenfruchtanbaues vor dem Maisanbau auf die folgenden Frühjahrs und Herbst $\mathrm{N}_{\min }$-Mengen sind die zur Verfügung stehenden Daten zum Zwischenfruchtanbau in „Brache“, und „Nicht Leguminose“ eingeteilt worden. Eine Beeinflussung der Frühjahrs und Herbst $\mathrm{N}_{\text {min }}$-Menge durch den Anbau einer „Nicht Leguminosen“ Zwischenfrucht gegenüber einer Brache kann mittels des $\mathrm{t}$-Tests nicht nachgewiesen werden. Nur von wenigen Flächen liegen Angaben zur Frühjahrs $\mathrm{N}_{\text {min }}$-Menge und zur Zwischenfrucht vor, deshalb beschränkt sich die Abb. 59 auf die Herbst $\mathrm{N}_{\min }$-Menge nach dem Anbau von Mais. Unterschiede in der ausgebrachten Düngermenge („mit Düngung“) zur Hauptfrucht Mais, je nach dem ob eine Zwischenfrucht vorhanden war oder nicht, können nicht festgestellt werden.
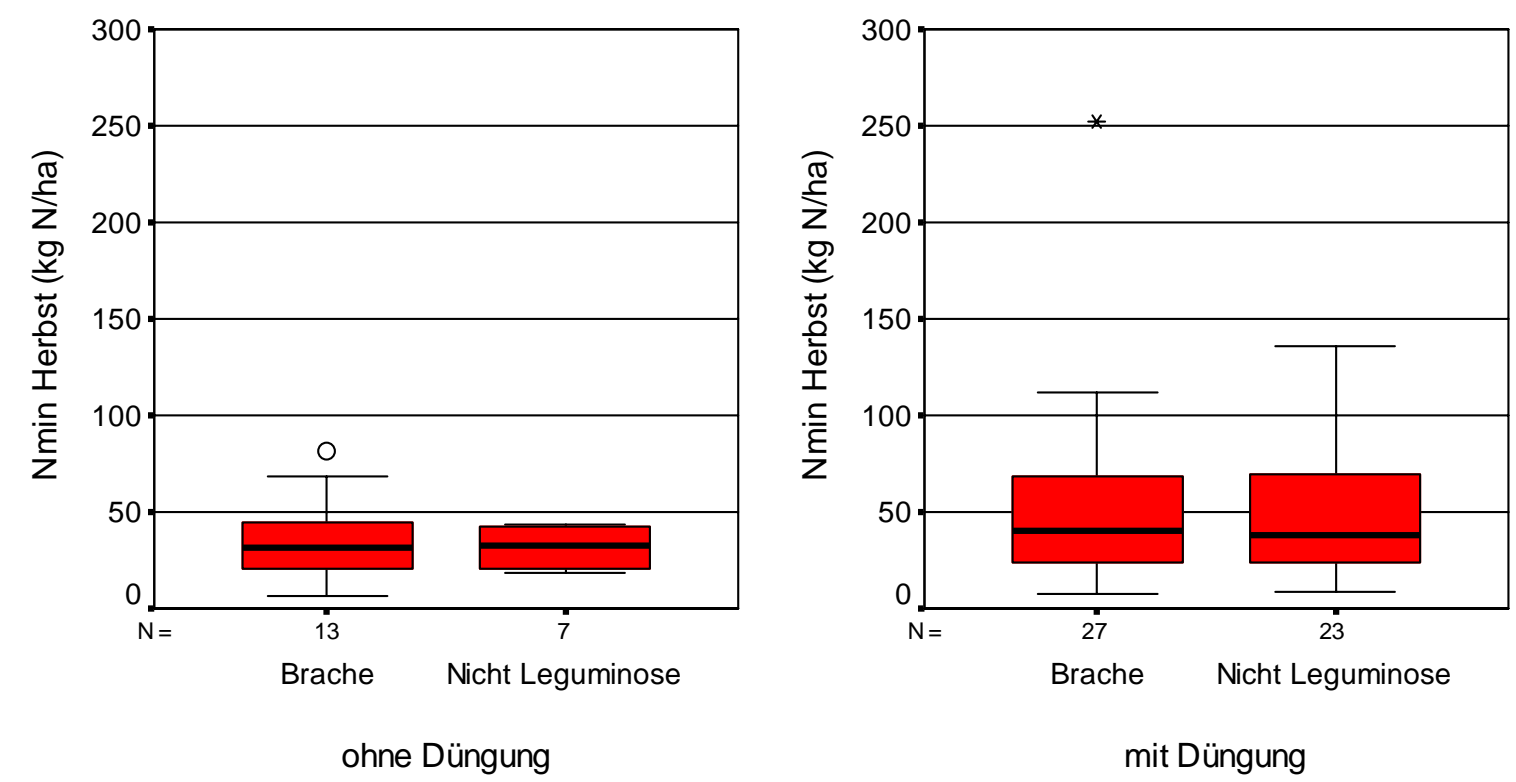

Abb. 59: Verteilung der Herbst $\mathrm{N}_{\min }$-Gehalte (kg N/ha) in Abhängigkeit von der „Brache“ oder dem Einsatz einer „Nicht Leguminosen“ Zwischenfrucht vor dem Maisanbau (Datenkategorie 3)

Der Einfluss des Frühjahrs $\mathrm{N}_{\min }$-Gehaltes durch die Vorfrucht kann nicht untersucht werden, da die Anzahl der Werte zu gering ist. Der Einfluss der Vorfrucht auf den 
Herbst $\mathrm{N}_{\min }$-Gehalt wird in Abb. 60 dargestellt. In die Abbildung sind nur Vorfrüchte aufgenommen worden, die auf mindestens $5 \%$ der Flächen standen. Aus den Abbildungen geht hervor, dass die Unterschiede in den Herbst $\mathrm{N}_{\min }$-Gehalten weniger als $25 \mathrm{~kg} \mathrm{~N} / \mathrm{ha}$ im Median betragen. Die Herbst $\mathrm{N}_{\min }$-Menge nach dem Anbau von Mais ist bei den gedüngten und ungedüngten Flächen nach der Vorfrucht Weidelgras im Gegensatz zu den anderen Vorfrüchten höher. Eine statistische Untersuchung wird auf Grund der geringen Flächenanzahlen nicht durchgeführt.
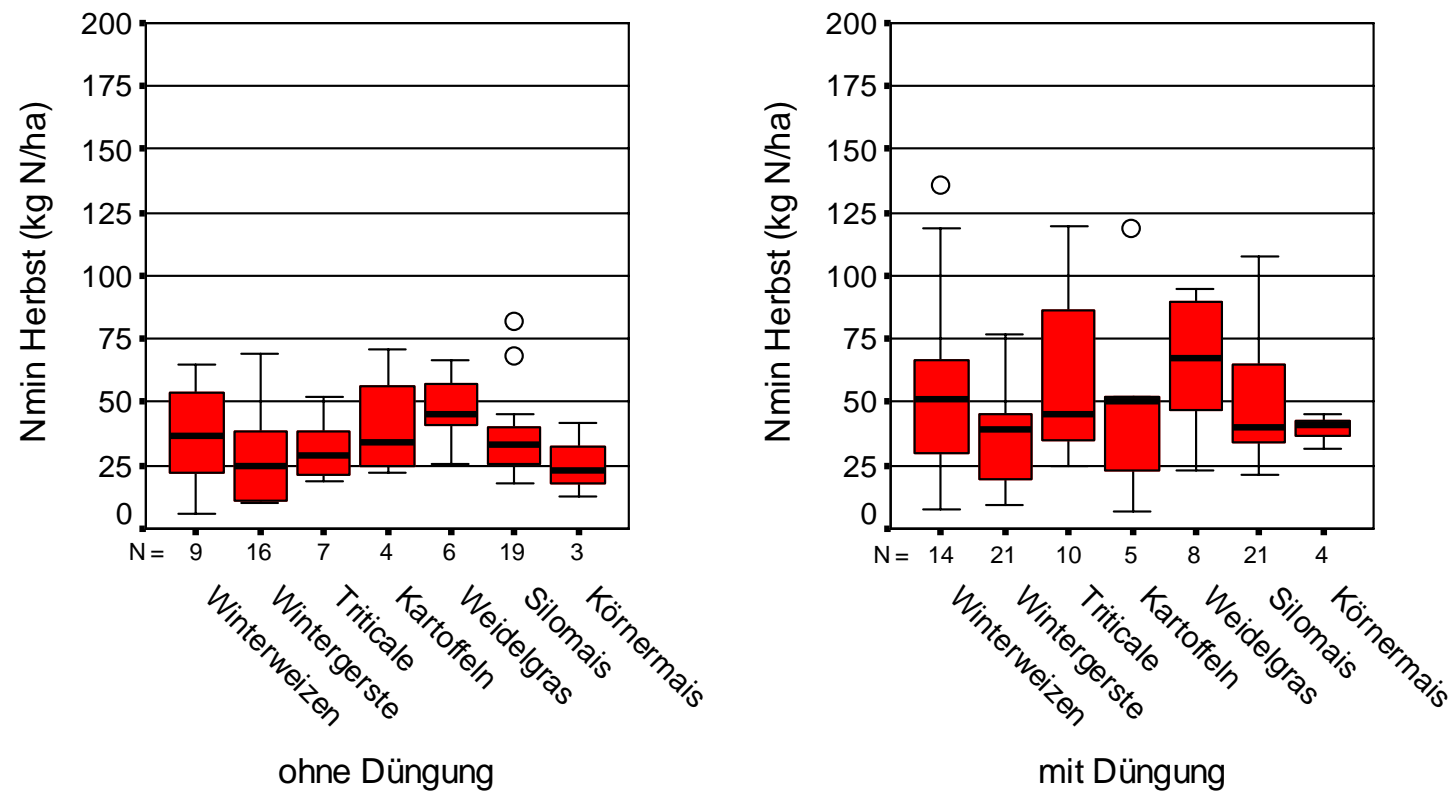

Abb. 60: Verteilung der Herbst $\mathrm{N}_{\min }$-Gehalte in Abhängigkeit von der Vorfrucht vor dem Maisanbau (Datenkategorie 3)

\section{Einfluss der Witterung auf die Herbst $\mathrm{N}_{\text {min }}$-Menge}

In der Tab. 25 ist kein Einfluss der Witterung auf den $\mathrm{N}_{\text {min }}$-Gehalt im Herbst festzustellen. Weder die Temperatur noch die Niederschläge in den Monaten vor der $\mathrm{N}_{\min }$-Beprobung zeigen signifikante Korrelationskoeffzienten auf. So kann auch keine Beziehung in den ungedüngten oder gedüngten Flächen mittels einer Korrelation zwischen den aufsummierten Niederschlägen von Mai bis einschließlich August oder September und den Herbst $\mathrm{N}_{\min }$-Gehalten nachgewiesen werden. 
Tab. 25: Korrelation (Kendall) zwischen der Witterung (Temperatur und Niederschlag) und dem Herbst $\mathrm{N}_{\min }$-Gehalt (0-90cm / Datenkategorie 3)

\begin{tabular}{|c|c|c|c|c|c|c|c|}
\hline & \multicolumn{6}{|c|}{ Monat vor der Beprobung } \\
\hline & & Mai & Juni & Juli & August & September & Oktober \\
\hline \multirow[t]{4}{*}{ Temperatur } & Ohne &,$- 001^{\text {(n.s.) }}$ & ,038 &, $122^{(\text {(n.S.) }}$ &, $101^{\text {(n.s.) }}$ &, $096^{\text {(n.S.) }}$ &,$- 333^{\text {(n.s.) }}$ \\
\hline & Düngung & $(n=58)$ & $(n=58)$ & $(n=58)$ & $(n=58)$ & $(n=38)$ & $(n=3)$ \\
\hline & &, $013^{(\text {n.s. })}$ &, $011^{\text {(n.s.́. }}$ &,$- 094^{(\text {n.s.s.) }}$ &,$- 090^{\text {(n.s.) }}$ &,$- 043^{\text {(n.s.) }}$ &, $000^{(\text {n.s.s. }}$ \\
\hline & Düngung & $(n=73)$ & $(n=73)$ & $(n=73)$ & $(n=73)$ & $(n=46)$ & $(n=4)$ \\
\hline \multirow[t]{4}{*}{ Niederschlag } & Ohne &, $018^{(\text {n.s. })}$ &,$- 139^{\text {(n.s.) }}$ &,$- 108^{\text {(n.s.) }}$ &,$- 017^{\text {(n.s.) }}$ &,$- 144^{\text {(n.s.) }}$ &,$- 333^{\text {(n.s.) }}$ \\
\hline & Düngung & $(n=58)$ & $(n=58)$ & $(n=57)$ & $(n=57)$ & $(n=38)$ & $(n=3)$ \\
\hline & Mit &,$- 038^{(\text {n.s.s.) }}$ &,$- 032^{(\text {n.s.s.) }}$ &,$- 041^{\text {(n.s.s.) }}$ &, $057^{(\text {n.s. })}$ & $104^{(\text {n.s. })}$ &, $333^{\text {(n.s.s.) }}$ \\
\hline & Düngung & $(n=73)$ & $(n=73)$ & $(n=72)$ & $(n=72)$ & $(n=46)$ & $(n=4)$ \\
\hline
\end{tabular}




\section{Diskussion}

Das Ziel dieser Arbeit war es, durch die Zusammenführung und gemeinsame Verrechnung einer großen Anzahl von Feldversuchen sowie von Daten, die im Rahmen der Wasserschutzgebietsberatung erhoben wurden, die Bedeutung wichtiger produktionstechnischer und standortbezogener Faktoren sowie deren Wechselwirkungen für die Nitrataustragsgefahr im Maisanbau zu analysieren. Dazu sind im Ergebnisteil drei Datenkategorien gebildet und deren Ergebnisse getrennt dargestellt worden. Dies geschah vor dem Hintergrund, dass auf Grund der Literaturaussagen und dem unterschiedlichen Kontext der Erhebungen von ungleichen Ergebnissen zwischen den Daten aus der Praxis und den Exaktversuchen ausgegangen worden ist. Des Weiteren wurde erwartet, durch die verschiedenen Datensätze die auftretenden Effekte bzw. Interaktionen besser eingrenzen zu können.

Die Diskussion erfolgt nun kategorieübergreifend. Es werden die Einflüsse der Düngermenge, des Viehbesatzes, des N-Saldo, des Standortes und der Fruchtfolgegestaltung auf den Herbst $\mathrm{N}_{\min }$-Gehalt unter Berücksichtigung von Interaktionen diskutiert. Den Abschluss bildet eine Diskussion über die Aussagekraft von Erhebungen (Praxisdaten) im Vergleich zu Exaktversuchen.

\subsection{Einfluss der Düngermenge auf den Herbst $\mathrm{N}_{\min }$-Gehalt im Standortkontext}

Der Düngung wird ein großer Einfluss auf die Nitratkontamination der Grundwässer zugeschrieben (WANTULLA et al. 1988). Eine nicht bedarfsgerechte Düngung eines Pflanzenbestandes kann zu einem zunehmenden Nitrateintrag in das oberflächennahe Grundwasser führen (AMBERGER, 1983; SCHWEIGER, 1989; BRANDHUBER und HEGE, 1991b; BENKE et al., 1999; GOULDING, 2000). Insbesondere eine überhöhte Düngung des Maises kann hohe Rest $\mathrm{N}_{\min }$-Mengen und Nitratauswaschungen verursachen (LORENZ et al., 1991; JOKELA, 1992; GEBAUER und SCHAAF, 1993; WIEKER und LÜTKE ENTRUP, 1995). ROUVÉ und BOGACKI (1987); STICKSEL et al. (1994b) und TIMMERMANN (1994) stellten fest, dass durch die Reduzierung des Stickstoffeinsatzes die Nitratgehalte nach der Maisernte zurückgehen. Dementsprechend fanden VERBRUGGEN et al. (1998) 
einen Anstieg der $\mathrm{N}_{\min }$-Gehalte nach der Ernte in Abhängigkeit von der Höhe der Düngung vor. Hingegen zeigte sich in den Untersuchungen von WANTULLA (1987) auf bindigen Lößlehmböden nicht generell ein Einfluss der Düngung auf den Herbst

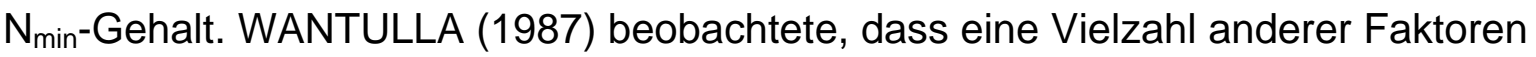
die düngungsspezifischen Unterschiede überdeckten. Nach CZERATZKI (1973) hat die aktuelle N-Düngung nur eine geringe Bedeutung auf den N-Haushalt und die Nitratverlagerung. Dass der Herbst $\mathrm{N}_{\min }$-Gehalt nicht mit der $\mathrm{N}$-Düngung im Jahr der Probenahme korreliert, stellte KETELSEN (2001) auf Auenlehmen und Lößböden fest. RIEß (1993) konstatierte in seinen Untersuchungen auf schwach pseudovergleyter Parabraunerde aus Lößlehm, dass die Düngung nur einen relativ geringen Eingriff in die N-Dynamik des Bodens darstellt. Die natürliche Mineralisation der organischen Substanz und die jahresspezifischen Witterungsverhältnisse überlagerten nach RIEß (1993) den Düngungseffekt stark. In den hier durchgeführten Analysen kann kein genereller Einfluss der Höhe der mineralischen oder organischen Düngung auf die Herbst $\mathrm{N}_{\min }$-Gehalte in den Datenkategorien nachgewiesen werden. Nach WANTULLA et al. (1988) und KETELSEN (2001) wäre es auf Grund der sehr komplexen Dynamik des Stickstoffs im Boden keinesfalls ungewöhnlich, dass die $\mathrm{N}_{\min }$-Gehalte nach der Ernte weder mit der Menge noch dem Zeitpunkt der N-Düngung in unmittelbarem Zusammenhang stehen.

Dass die Düngung keinen Einfluss auf den vorgefundenen $\mathrm{N}_{\min }$-Gehalt im Herbst hat, kann bei einer separaten Betrachtung der einzelnen gedüngten Exaktversuche aus der dritten Datenkategorie nicht generell bestätigt werden. Hier zeigen sich in verschiedenen Versuchen Einflüsse der ausgebrachten Düngermenge auf den vorgefundenen $\mathrm{N}_{\min }$-Gehalt im Herbst. Da das Ausmaß der düngebedingten Zunahme des Herbst $\mathrm{N}_{\min }$-Gehaltes der Einzelversuche sehr wahrscheinlich nicht groß genug ist, um die starken Unterschiede der Einzelstandorte im Hinblick auf das Grundniveau des Herbst $\mathrm{N}_{\min }$-Gehaltes zu dominieren, scheint die Zusammenlegung der unterschiedlichen Einzelstandorte zu dem vorgefundenen diffusen Bild zu führen. Inwieweit die Herbst $\mathrm{N}_{\min }$-Gehalte streuen ist besonders gut bei den ungedüngten Exaktversuchen zu sehen. Deshalb ist geprüft worden, ob Standorteffekte den möglichen Einfluss der Düngung bei der Verrechnung in Datenkategorien überlagerten. 
Zunächst wurde der Einfluss der Bodenart auf den $\mathrm{N}_{\min }$-Gehalt im Herbst untersucht, da die Herbst $\mathrm{N}_{\min }$-Gehalte sich in den Untersuchungen von KLINGER und TIMMERMANN (1988) je nach Bodenart unterscheiden. Auch STURM und ISERMANN (1987) beobachten ausgeprägte Standorteinflüsse auf den Restnitratgehalt nach der Ernte. RÜCK und STAHR (1996) fanden hingegen auf 61 Flächen kaum einen Einfluss der Bodenart auf den Herbst $\mathrm{N}_{\min }$-Gehalt vor.

Eine Differenzierung der Herbst $\mathrm{N}_{\min }$-Mengen zwischen den Bodenarten ist in der vorliegenden Arbeit nur in den Daten der Kategorie 2 nachzuweisen. Die Verteilung der eingeflossenen Bodenarten (leicht - schwer) war in dieser Datenkategorie am gleichmäßigsten. Die Herbst $\mathrm{N}_{\text {min }}$-Gehalte liegen im Mittel der leichten Standorte geringfügig über denen der sorptionsstärkeren Standorte. Hierbei ist aber die größere Streuung innerhalb der leichten Standorte zu beachten, was darauf hindeutet, dass weitere, nicht eng mit der Bodenart verknüpfte Faktoren Einfluss auf die Herbst $\mathrm{N}_{\text {min }}{ }^{-}$ Gehalte nehmen. In den anderen Datenkategorien liegen die $\mathrm{N}_{\min }$-Gehalte im Herbst nahezu auf gleicher Höhe. Ein Einfluss der Bodenarten auf den Zusammenhang zwischen der gesamten Düngermenge und dem Herbst $\mathrm{N}_{\min }$-Gehalt kann in keiner der drei Datenkategorien festgestellt werden. Weder die leichten noch die schweren Standorte zeigen eine spezifische Einflussnahme. Selbst sehr hohe Düngermengen vermochten keinen gerichteten Einfluss auf den $\mathrm{N}_{\min }$-Gehalt im Herbst auszuüben. Somit lässt sich festhalten, dass auf den verschiedenen Standorten weitere Faktoren einen Einfluss auf den Grad der Auswirkung der N-Düngung und den folgenden $\mathrm{N}_{\min }{ }^{-}$ Gehalt im Herbst haben.

\subsection{Einfluss des Flächenbilanzsaldos auf den Herbst $\mathrm{N}_{\text {min }}$-Gehalt}

Der Boden nimmt die Stickstoffeinträge durch Dünger, durch die Atmosphäre und durch die biologische N-Fixierung auf (JARVIS et al., 1996). Dieser Stickstoff kann den angebauten Früchten dienen oder aber auch verloren gehen (z.B. durch Auswaschung) (JARVIS et al., 1996).

In der Nährstoff-Einzelschlagbilanz wird die Nährstoffzufuhr zu einer Fläche der Nährstoffabfuhr gegenübergestellt (ANTONY et al. 2001). Das Ergebnis der Bilanz beeinflusst längerfristig den Bodenvorrat an Nährstoffen (KLASINK und STEFFENS, 1997). Eine Bilanz lässt nach KÖPPEN (1997) Rückschlüsse über die Effizienz der organischen und mineralischen Düngung, sowie die Nährstoffverluste zu. Als 
Maßnahme zum Nachweis einer sachgerechten Düngung kann somit die vereinfachte N-Bilanz (N-Saldoberechnung) dienen (BAUMGÄRTEL et al., 1989). Das Gefährdungspotential einer N-Auswaschung kann nach HERSEMANN und LAMMEL (1990) auf der Basis einer flächenbezogenen Stickstoffbilanz verdeutlicht werden. Die Einzelschlagbilanz ermöglicht eine Abschätzung der langfristigen Auswirkung der Bewirtschaftungsweise auf die Sickerwasserqualität (ANTONY et al., 2001). Eine Beziehung zwischen dem N-Saldo und der Nitratkonzentration im Sickerwasser unter Maismonokultur stellten BOBE et al. (2003) fest. In den Untersuchungen von WEISE und SCHELLER (1997) weisen die Schläge mit niedrigen $\mathrm{N}$-Überschüssen in der Flächenbilanz auch niedrige $\mathrm{N}_{\min }$-Gehalte nach der Ernte auf. Diese Aussage beschränkt sich allerdings auf Böden, deren Gehalt an organischer Substanz nicht sehr hoch ist. Eine enge Beziehung zwischen einer vereinfachten Bilanz, dem $\mathrm{N}_{\text {min }}$-Gehalt des Bodens nach Vegetationsende und der Nitratkonzentration im Sickerwasser stellten HEROLD et al. (1996) fest. Hingegen sehen APPEL et al. (1993) in der schlagbezogenen N-Bilanz kein geeignetes Instrument zur Beurteilung der Nitratauswaschungsgefahr. Insbesondere bei der Fruchtart Mais lässt die N-Bilanz nach LORENZ und STEFFENS (1997) keine Aussage über die Höhe der Auswaschung zu. Der Herbst $\mathrm{N}_{\min }$-Gehalt korreliert nach Ansicht von KETELSEN (2001) und ANTONY (1993) nicht oder nur schwach mit den schlagbezogenen N-Salden im Jahr der Probenahme.

Eine Beziehung zwischen dem N-Saldo und dem $\mathrm{N}_{\min }$-Gehalt ist in dieser Untersuchung nur in der Datenkategorie 3 auf den gedüngten Flächen, in Form einer sehr schwachen positiven Korrelation, nachzuweisen. Aus den anderen Daten der Kategorie 1, 2 und den ungedüngten Flächen der Kategorie 3 ist keine entsprechende Aussage abzuleiten, da die Streuung zu stark und nicht gleichgerichtet ist. Die Aufnahme des N-Saldos als Variable in die Komponente 1 der Faktorenanalyse bei den ungedüngten Flächen der Datenkategorie 3 ist dadurch zu erklären, dass der N-Saldo mit der Bodenbonität einhergeht. Da keine Düngung appliziert wurde, lässt sich in diesem Fall der N-Saldo mit dem N-Entzug gleichsetzen. Über die Bodenqualität, welche in den anderen bodenbezogenen Variablen dieser Komponente ihren Niederschlag findet, wird das Wachstum der Maispflanze und damit der Ertrag und folglich der Entzug beeinflusst. Daher besteht eine Beziehung zwischen dem N-Saldo und den anderen ausgewiesenen Variablen dieser Komponente der Faktorenanalyse. 
Die vorgenommene Differenzierung der vorliegenden Daten nach Bodenartgruppen erbringt in keiner der Datenkategorien eine weitere Klärung der Streuungsursachen. Eine nicht hinreichende Qualität der hier lediglich kategorial erfassten Daten aus der Wasserschutzgebietsberatung ist als eine mögliche Streuungsursache nicht auszuschließen. Da aber selbst in den Erhebungen und auf den ungedüngten Flächen der Kategorie 3 mit exakten Messwerten keine direkte Beziehung zwischen dem $\mathrm{N}$-Saldo und dem $\mathrm{N}_{\min }$-Gehalt nach der Ernte zu finden ist, dürfte die Ursache eher in dem N-Nachlieferungspotential des Standortes zu suchen sein. Auch APPEL et al. (1993) sehen den Grund für eine unzureichende Aussagekraft schlagbezogener N-Bilanzen in der Variabilität des N-Nachlieferungspotentials der Standorte.

\subsection{Rolle des Viehbesatzes und Einfluss der Düngerart auf den Herbst $\mathrm{N}_{\text {min }^{-}}$ Gehalt}

Der Viehbesatz steht in enger Beziehung zu den anfallenden Exkrementen der Tiere. Es wird ein Großteil des mit dem Futter aufgenommenen Stickstoffs von den Tieren wieder ausgeschieden. Die Exkremente weisen je nach Tierart, der Fütterung und dem Wassergehalt unterschiedliche Stickstoffkonzentrationen bei der Düngung auf. Nach HOEGEN und WERNER (1998) werden insbesondere auf den mit Gülle gedüngten Praxisflächen höhere Rest $\mathrm{N}_{\min }$-Gehalte gefunden als auf den mineralisch gedüngten. Hohe Viehbesätze verursachen nach HARTMANN (1993) steigende Nitratgehalte im Grundwasser. ERLENBACH (1987) sieht in einer hohen Viehbesatzstärke negative Folgen für den Grundwasserschutz. In viehlosen Betrieben wird nach HEGE und BRANDHUBER (1990) vielfach intensiver Ackerbau ohne übermäßige Nitratauswaschung praktiziert. Aus ihren Untersuchungen leiten ORTSEIFEN und SCHEFFER (1997) ab, dass zur Entlastung der Gewässer mit Nitrat zunächst die Gebiete mit intensiver Viehhaltung auf marginalen Standorten extensiviert werden sollten. Hingegen besteht bei BRANDHUBER und HEGE (1991b) zwischen dem Viehbesatz und der Nitratkonzentration im Sickerwasser kein Zusammenhang. Nach SOLANSKY (1987) berücksichtigen die viehhaltenden Betriebe den Güllestickstoff und reduzieren dementsprechend den Einsatz von mineralischen Düngern zu Mais. 
In der eigenen Untersuchung ist ein etwaiger Einfluss der Viehbesatzstärken auf die Herbst $\mathrm{N}_{\min }$-Gehalte in keiner der untersuchten Datenbestände nachzuweisen. Eine Korrelation der Nitratgehalte unter Schlägen mit Wirtschaftsdüngung und dem Viehbesatz konnten auch HEGE und BRANDHUBER (1990) nicht feststellen. Dass die Bodenart die Auswirkungen des Viehbesatzes auf den $\mathrm{N}_{\min }$-Gehalt im Herbst beeinflusst, ist anhand der vorliegenden Datenlage nicht nachzuvollziehen. In der Datenkategorie 1 befinden sich hauptsächlich leichte Böden, während in Datenkategorie 2 mittelschwere Böden überwiegen. Dass Betriebe mit hohen Viehbesatzstärken von mehr als $2 \mathrm{GV} / \mathrm{ha}$ weniger mineralischen Dünger ausbringen, ist in den Daten aus der Wasserschutzgebietsberatung in der Tendenz erkennbar. In den Daten der Kategorie 2 ist dagegen kein Einfluss der Viehbesatzstärke auf die mineralische Düngermenge festzustellen. Die ausgebrachte organische Düngermenge in diesen beiden Datenkategorien ist von der Höhe des Viehbesatzes abhängig. Mit höheren Viehbesätzen nimmt auch die applizierte organische Düngermenge zur Fruchtart Mais zu. Die von etlichen Autoren aufgeführten Zusammenhänge zwischen der Viehbesatzdichte und dem Nitrataustrag lassen sich demnach nur durch eine überproportional hohe Ergänzungsdüngung plausibel erklären. Da die gesamte Düngermenge aber keinen gerichteten Einfluss auf den Herbst $\mathrm{N}_{\min }$-Gehalt zeigt, wird im Folgenden untersucht, ob die Art der ausgebrachten Düngung zu einer weiteren Klärung beitragen kann. Eine Aufteilung der gesamten Düngermenge nach den Anteilen an mineralischen und organischen Düngemitteln zeigt, dass die mineralische Düngung in der Datenkategorie 1 eher als Ergänzung der organischen Düngung betrachtet wird. Hingegen wird in der Datenkategorie 2 auf vielen Flächen rein mineralisch gedüngt. Die Exaktversuche der Datenkategorie 3 werden hauptsächlich mineralisch gedüngt. In Bezug zum Herbst $\mathrm{N}_{\text {min }}$-Gehalt erbringt die Aufteilung der gesamten Düngermenge nur in den Daten der Kategorie 2 eine weitere Erklärung. Der $\mathrm{N}_{\min }$-Gehalt im Herbst zeigt in der Datenkategorie 2 die Tendenz mit zunehmendem Anteil der organischen Düngung zu steigen. Es konnte mittels Korrelationsanalyse diese Tendenz aber nicht signifikant $(p=0,051)$ belegt werden. In der Faktorenanalyse und der multiplen Regression zeigen sich hier zwar schwache, jedoch statistisch gesicherte Zusammenhänge auf.

Die Betrachtung der rein mineralisch oder rein organisch gedüngten Flächen zeigt in keiner der 3 Datenkategorien eine Beziehung zum Herbst $\mathrm{N}_{\min }$-Gehalt. Deshalb lässt 
diese Untersuchung keine Aussage darüber zu, ob die alleinige organische oder die alleinige mineralische Düngung einen höheren Einfluss auf den Herbst $\mathrm{N}_{\min }$-Gehalt hat. Nach HOEGEN und WERNER (1998) werden insbesondere auf den mit Gülle gedüngten Praxisflächen höhere Rest $\mathrm{N}_{\min }$-Gehalte gefunden als auf den mineralisch gedüngten. Da diese Feststellung auch mit dem Einfluss der organischen Düngung auf die N-Nachlieferung des Bodens zusammenhängen kann, wurden in der Vergangenheit viele Untersuchungen zu den Auswirkungen der applizierten Dünger auf die Veränderung des N-Nachlieferungsvermögens der Böden und die damit verbundenen N-Verluste gemacht (SCHRÖDER et al., 1986). Auf organisch gedüngten Flächen werden nach HECHT et al. (1980) größere N-Mengen freigesetzt als auf mineralisch gedüngten Flächen. Das N-Nachlieferungspotential wird laut WERNER et al. (1985) durch jahrelange Gülledüngung erhöht. Hohe Stallmistgaben führen nach HÜLSBERGEN et al. (1992) sowie BALIK und OLFS (1998) zu beträchtlichen $\mathrm{N}_{\mathrm{t}}$-Anreicherungen. Nach ALBERT (2001) und HAO et al. (2003) wird durch organische Düngemittel der Gesamt-N Gehalt des Bodens in der Krume und damit das schwer zu kalkulierende N-Nachlieferungs- und Auswaschungspotential erhöht. Auch in den Daten aus der Wasserschutzgebietsberatung kann eine schwache positive Korrelation zwischen der Menge an ausgebrachter organischer Düngung und der Höhe der Gesamt-N Gehalte festgestellt werden. Dieser Befund könnte darauf zurückzuführen sein, dass die betreffenden Flächen schon von jeher höher mit organischer Düngung versorgt worden sind. Andererseits konnte in den Versuchen von WERNER et al. (1985) und RICHTER et al. (1997) der $\mathrm{N}_{\mathrm{t}}$-Gehalt des Bodens durch unterschiedliche Düngung mit mineralischen oder organischen Düngern nicht beeinflusst werden. Auch KÖRSCHENS (1998a) stellte in 18 Jahren nur sehr geringe Veränderungen im $\mathrm{N}_{\mathrm{t}}$ und $\mathrm{C}_{\text {org }}$-Gehalt durch extreme Änderungen im Düngeregime fest. Da die Struktur der Daten in der Kategorie 1 eine Trendanalyse nicht zulässt, muss es hier offen bleiben, ob die aktuelle organische Düngung und die $\mathrm{N}_{\mathrm{t}}$-Gehalte des Bodens in einem ursächlichen Zusammenhang stehen. In der ersten und zweiten Datenkategorie kann keine Beziehung zwischen den $\mathrm{C}_{\mathrm{t}^{-}} / \mathrm{N}_{\mathrm{t}^{-}}$ Gehalten des Bodens und dem Viehbesatz festgestellt werden. In der dritten Datenkategorie liegen keine Angaben zum Viehbesatz vor. 


\subsection{Einfluss mineralisationsrelevanter Bodenmerkmale $\left(C_{t}, N_{t}, C / N\right.$, Frühjahrs $\mathbf{N}_{\min }$ ) auf den Herbst $\mathbf{N}_{\min }$-Gehalt}

Die organische Substanz des Bodens wird durch den $\mathrm{C}_{\mathrm{t}^{-}}$und $\mathrm{N}_{\mathrm{t}}$-Gehalt des Bodens charakterisiert (KÖRSCHENS, 1992). Durch die organische Substanz werden nahezu alle bodenbiologischen und die Mehrzahl aller bodenchemischen Prozesse bestimmt. Sie ist somit ein wesentliches Merkmal der Bodenfruchtbarkeit (KÖRSCHENS, 1992). Die Gesamt-N Gehalte der hier untersuchten Böden liegen vorwiegend zwischen 0,1 und $0,2 \%$. Dieses ist nach SCHEFFER und SCHACHTSCHABEL (2002) der übliche Bereich von Mineralböden im gemäßigten humiden Klima. Die $\mathrm{C}_{t}$-Gehalte variierten überwiegend zwischen 1 und $3 \%$.

Nach NIEDER und RICHTER (1986) sehen viele Autoren die Korngrößenzusammensetzung des Bodens als den wichtigsten Standortfaktor, der einen Einfluss auf den Humusgehalt hat, an. Die Ergebnisse von BROGOWSKI et al. (1974) weisen darauf hin, dass der Humusgehalt mit zunehmenden Korngrößen abnimmt. KÖRSCHENS (1998b) stellte in 13 Europäischen Versuchen eine enge Beziehung des Kohlenstoff- und Gesamt-N Gehaltes mit dem Tonanteil fest. Die Gehalte an Kohlenstoff und Gesamt-N im Boden steigen nach KÖRSCHENS (1998b) mit zunehmendem Tonanteil. Dass der Kohlenstoffgehalt mit zunehmenden Tonanteil im Boden generell ansteigt, kann durch die eigene Untersuchung nicht bestätigt werden. Die Daten aus der Wasserschutzgebietsberatung und den Erhebungen zeigen keine Tendenz auf. Lediglich der Gesamt-N Gehalt des Bodens nimmt in den Daten aus den Erhebungen mit zunehmendem Tonanteil zu. Auch SPRINGOB (2003) kann die Zunahme der Kohlenstoffgehalte in Abhängigkeit vom Tonanteil, insbesondere für Sandböden, nicht bestätigen. Nach SPRINGOB (2003) nehmen die C-Gehalte, bedingt durch zunehmende Anteile an schwer abbaubaren organischen Substanzen, in Norddeutschland von Ost nach West zu. Dies sei verknüpft mit der Podsolierung, Heidebildung, Plaggennutzung sowie rezenten Grundnässeeinflüssen und habe mit dem Ton nur insofern zu tun, als dessen Abwesenheit die Bildung solcher Materialien begünstige.

Die Fähigkeit eines Bodens, den N-Bedarf der Maispflanze über eine Wachstumsperiode durch $\mathrm{N}$-Mineralisierung zu decken, ist abhängig von der organischen N-Reserve im Boden, der Natur der organischen Rückstände und der Mineralisationskapazität der Bodenorganismen. Der bodenbürtige Stickstoff 
verursacht laut WANTULLA et al. (1988) eine Grundbelastung der Bodenlösung mit Nitrat. Nach FEIGE und RÖTHLINGSHÖFER (1990) liegt eine der Hauptursachen für den zunehmenden Nitrataustrag in den gestiegenen Bodenstickstoffgehalten. Zwischen dem $\mathrm{N}_{\mathrm{t}}$-Gehalt und dem N-Nachlieferungsvermögen des Bodens besteht eine enge Beziehung (HECHT et al., 1980). In den eigenen Untersuchungen besteht zwischen den $\mathrm{N}_{\text {min }}$-Mengen im Herbst und dem $\mathrm{N}_{\mathrm{t}}$-Gehalt des Bodens bei allen Datenkategorien ein markanter Zusammenhang. Dieser zeigt sich insbesondere auf den nicht gedüngten Parzellen der Exaktversuche.

Die Faktorenanalyse der Daten aus den Exaktversuchen gruppiert den Gesamt-N Gehalt und den $\mathrm{C}_{\mathrm{t}}$-Gehalt des Bodens in die gleiche Komponente. Diese Komponente weist die beste Beziehung zum Herbst $\mathrm{N}_{\min }$-Gehalt auf. Die vorgenommene Gruppierung der Faktorenanalyse ist mit der engen Beziehung dieser beiden, den Boden beschreibenden Merkmale zu erklären. So korreliert der Gehalt an organisch gebundenem Stickstoff im Boden eng mit dem $\mathrm{C}_{\mathrm{t}}$-Gehalt (KÖRSCHENS, 1992; NEU, 1999). Generell ist der Stickstoffhaushalt der Böden eng mit dem Kohlenstoffhaushalt gekoppelt (FRANKO et al., 2001). Laut ZIEGLER (1991) nimmt der Nitratstickstoff bei Vegetationsende mit dem Humusgehalt des Bodens zu. PFEFFERKORN (1993) wies auf den Zusammenhang hin, dass bei hohen $\mathrm{C}_{\mathrm{t}^{-}}$ Gehalten auch stark erhöhte $\mathrm{NO}_{3}$-Gehalte im Wurzelraum auftreten. Hohe Kohlenstoffgehalte im Boden erhöhen damit die Gefahr von N-Verlusten (KÖRSCHENS, 1999).

In der eigenen Untersuchung kann in allen drei Datenkategorien ein signifikanter Zusammenhang zwischen dem $\mathrm{C}_{\mathrm{t}}$-Gehalt und dem Herbst $\mathrm{N}_{\text {min }}$-Gehalt und bis auf die gedüngten Exaktversuche auch zwischen dem $\mathrm{N}_{\mathrm{t}}$-Gehalt und dem Herbst $\mathrm{N}_{\text {min }}$ Gehalt festgestellt werden. In der Regression stellt sich dann mal der $C_{t}$ und mal der Gesamt-N Gehalt als das bessere Charakteristikum für die Beschreibung des Zusammenhanges der organischen Substanz mit dem Herbst $\mathrm{N}_{\text {min }}$-Gehalt dar. Dieses lässt sich mit der engen Korrelation des in der organischen Substanz des Bodens gebundenen Gesamt-N mit dessen C-Gehalt erklären (NEU, 1999).

Die N-Freisetzung ist laut HECHT et al. (1980) nicht nur von der Höhe der $\mathrm{N}_{\mathrm{t}^{-}}$und $\mathrm{C}_{\mathrm{t}^{-}}$ Gehalte im Boden abhängig, sondern auch von Unterschieden in der organischen Substanz bezüglich ihrer Mineralisierbarkeit.

Der klassische Indikator für die Humusqualität und ein Maß für den Abbaugrad der organischen Bodensubstanz ist das C/N-Verhältnis (CAPRIEL, 2003). Das C/N- 
Verhältnis wird in den Erhebungen und Exaktversuchen von der leichten Bodengruppe (<12\% Tonanteil) zu den schweren Bodengruppen ( $>12 \%$ Tonanteil) enger. Aus einem engen C/N-Verhältnis resultiert nach HUGGER (1992), SCHEFFER und SCHACHTSCHABEL (2002) sowie MÜLLER-THOMSON et al. (1998) eine hohe biologische Aktivität, die zu einer starken Stickstoffmineralisation führt. Eine entsprechende Beziehung zwischen dem C/N-Verhältnis und den im Herbst vorgefundenen $\mathrm{N}_{\text {min }}$-Mengen kann nur in der Datenkategorie 2 und speziell für die $\mathrm{N}_{\text {mon}}$-Projekt Daten festgestellt werden. Sogar auf den ungedüngten Flächen der Datenkategorie 3 ist keine Beziehung festzustellen.

Nach SPRINGOB (2003) stellt ein C/N-Verhältnis von ca. 15 bei Sandböden eine Schwelle dar. Wird es weiter, dann scheint die N-Freisetzung reduziert. Wird es enger, steigt die N-Freisetzung rasant an. Eine starke $\mathrm{N}$-Freisetzung bei $\mathrm{C} / \mathrm{N}$ Verhältnissen, die enger als 15 sind, lässt sich in den Daten der Wasserschutzgebietsberatung (insbesondere Sandböden) anhand der herbstlichen $\mathrm{N}_{\min }$-Gehalte nicht nachvollziehen. Bei $\mathrm{C} / \mathrm{N}-$ Verhältnissen größer 15 ist aber eine Tendenz zu niedrigeren Herbst $\mathrm{N}_{\min }$-Gehalten zu erkennen. Auch in den Daten aus den Exaktversuchen, welche nicht nur Sandböden enthalten, zeigt sich ein ähnlicher Effekt.

Der Herbst $N_{\min }$-Gehalt korreliert nach KETELSEN (2001) mit den Frühjahrs $N_{\min }{ }^{-}$ Gehalten im gleichen Jahr, wodurch das schlagspezifische Ausmaß der NNettomineralisation zum Ausdruck kommen dürfte. Die Korrelation der Frühjahrs $\mathrm{N}_{\min }$-Gehalte mit den Herbst $\mathrm{N}_{\min }$-Gehalten kann auch in allen drei Datenkategorien dieser Untersuchung vorgefunden werden. Insbesondere die Frühjahrs $\mathrm{N}_{\text {min }}$-Mengen, die nach dem 1. Mai erhoben wurden, korrelieren stärker mit den herbstlichen $\mathrm{N}_{\text {min- }}$

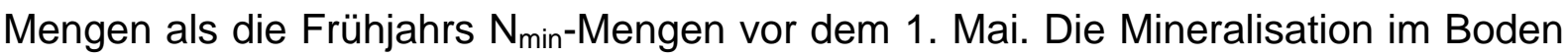
wird dementsprechend durch die spätere $\mathrm{N}_{\min }$-Beprobung im Frühjahr besser erfasst. Dieses stellten auch RICHARDS et al. (1999) fest. 


\subsection{Einfluss der Fruchtfolgegestaltung vor dem Maisanbau auf den Frühjahrs und Herbst $\mathbf{N}_{\min }$-Gehalt}

\section{Zwischenfrucht}

Die Gründe für den Zwischenfruchtanbau sind heute hauptsächlich in der Verminderung von Bodenerosion und der Reduktion des Nitrataustrages in Verbindung mit der N-Konservierung zu sehen (MAIDL und AIGNER, 1998). Insbesondere über die Wintermonate brachliegende Flächen neigen zur Auswaschung von Nährstoffen in tiefere Bodenschichten (EICHLER und ZACHOW, 2002; MERBACH et al., 1997; MERBACH und WURBS, 1997).

Die als Zwischenfrucht eingesetzten Fruchtarten haben nach EICHLER und ZACHOW (2002) einen signifikanten Einfluss auf die N-Aufnahme. Speziell die kruziferen Fruchtarten (Ölrettich, Senf, Raps, Rübsen, Raphanobrassica), aber auch Phacelia und Gräser nehmen sehr hohe Mengen an Stickstoff aus dem Boden auf (LÜTKE ENTRUP und ZERHUSEN, 1992; EICHLER und ZACHOW, 2002). Die Reihenfolge der N-Aufnahme ist nach MAIDL und AIGNER (1998) bei Phacelia < Winterraps < Ölrettich und < Senf. Der Einfluss der N-Aufnahme und der daraus resultierenden $\mathrm{N}$-Freisetzung der Zwischenfrüchte auf die vorgefundenen $\mathrm{N}_{\min }{ }^{-}$ Gehalte im Frühjahr und im Herbst wird im Folgenden diskutiert.

Anhand der Frühjahrs $\mathrm{N}_{\text {min }}$-Mengen in den Wasserschutzgebietsdaten ist $\mathrm{zu}$ erkennen, dass die Böden unter den abfrierenden Zwischenfrüchten Senf und Ölrettich, die vor der Hauptfrucht Mais angebaut wurden, höhere $\mathrm{N}_{\min }$-Gehalte aufweisen als die winterharten Zwischenfrüchte. Daher scheint es, dass die abfrierenden Zwischenfrüchte (Senf, Ölrettich) unmittelbar nach ihrem frostbedingten Absterben bei nachfolgend mild-feuchter Witterung beträchtliche Mengen des fixierten Stickstoffs wieder frei setzen und dadurch höhere $\mathrm{N}_{\text {min }}$-Gehalte im Frühjahr bewirken. Diese Feststellung wird auch in den Versuchen von BERGER et al. (1993), BERGER et al. (1996), BECKER at al. (1995) und VAN DIJK et al. (1997) gemacht. Offensichtlich speichern die winterharten Zwischenfrüchte in dieser Untersuchung den im Herbst aufgenommen Stickstoff bis zur Aussaat des Maises, da die $\mathrm{N}_{\text {min }}{ }^{-}$ Mengen im Frühjahr niedriger sind. Auch BERGER et al. (1993) und BERGER et al. (1996) stellten fest, dass die winterharten Zwischenfrüchte den aufgenommen Stickstoff länger speichern und damit die N-Verlagerung im Vergleich zu den abfrierenden Zwischenfrüchten auf ein Minimum reduzieren. 
Auf den im Winter brachliegenden Flächen werden ähnlich hohe $\mathrm{N}_{\min }$-Mengen im Frühjahr gefunden wie bei den winterharten Zwischenfrüchten. Auch in der Datenkategorie 2 kann zwischen der Brache über Winter und dem Anbau von nicht legumen Zwischenfrüchten vor der Hauptfrucht Mais kein Unterschied in den Frühjahrs $\mathrm{N}_{\text {min }}$-Mengen festgestellt werden. Dies lässt auf Auswaschungsverluste unter den Flächen ohne Zwischenfruchtanbau über den Winter schließen. Bei EICHLER und ZACHOW (2002) zeigte der Anbau von verschiedenen Zwischenfrüchten hinsichtlich der $\mathrm{N}_{\min }$-Mengen im Frühjahr keine gesicherten Unterschiede auf. Die Variante „Brache“ lag von den $\mathrm{N}_{\min }$-Mengen mit den anderen Zwischenfrüchten im Frühjahr gleich auf, was nach EICHLER und ZACHOW (2002) auf hohe N-Verluste schließen lässt. Nach BOSSHART (1984) liegt nämlich die Nitratkonzentration des Sickerwassers unter brachliegenden Feldern wesentlich höher als von ständig bedeckten Flächen.

Ein signifikanter Einfluss der vorherigen Zwischenfrucht auf den Herbst $\mathrm{N}_{\min }$-Gehalt nach dem Maisanbau lässt sich in der Datenkategorie 1 nicht feststellen. Tendenziell scheint aber der freiwerdende Stickstoff von Winterraps den Herbst $\mathrm{N}_{\min }$-Gehalt nach dem Anbau von Mais in der Datenkategorie 1 leicht zu erhöhen. Somit könnte nach SCHRÖDER et al. (1996) das Problem der Stickstoffverluste durch den Anbau von Zwischenfrüchten vom Winter in die Folgekultur verschoben werden, da durch die NFreisetzung aus den Zwischenfrüchten ein genereller $\mathrm{N}$-Überschuss beim Mais bestehen kann. Insbesondere die genaue Kalkulation der N-Nachlieferung aus einer eingearbeiteten Zwischenfrucht ist nach THORUP-KRISTENSEN und NIELSEN (1998) zurzeit nicht möglich und eine Abschätzung schwierig. Die Düngung zur Hauptfrucht Mais auf den mit Zwischenfrüchten bestellten Flächen lag in der Datenkategorie 1 gegenüber den Brache Flächen um nahezu 30 kg N/ha höher. Der Mais wurde nach der Zwischenfrucht Winterraps gegenüber den anderen Zwischenfrüchten eher gering mit Stickstoff gedüngt, daher ist der höhere $\mathrm{N}_{\text {min- }}$ Gehalt im Herbst wahrscheinlich durch eine stärkere N-Nachlieferung aus der Zwischenfrucht zu erklären. Die Herbst $\mathrm{N}_{\min }$-Mengen nach dem Anbau von Mais sind in der Datenkategorie 2 beim vorherigen Anbau einer Zwischenfrucht gegenüber der Brache geringfügig niedriger. Dieses kann darauf beruhen, dass die Düngung zur Hauptfrucht Mais bei mit Zwischenfrüchten bestellten Flächen im Gegensatz zur Brache im Mittel um $30 \mathrm{~kg} \mathrm{~N} / \mathrm{ha}$ niedriger war. In den Exaktversuchen zeigen sich keine Beeinflussungen der Herbst $\mathrm{N}_{\text {min }}$-Mengen durch den Zwischenfruchtanbau vor 
Mais. Auch die ausgebrachten Düngermengen zur Hauptfrucht Mais sind nicht signifikant unterschiedlich.

\section{Vorfrucht}

Jede Frucht hinterlässt in Abhängigkeit von der Art, Dauer der Vegetationszeit, Intensität des Einsatzes ertragssichernder Produktionsmittel unterschiedlich große Mengen an Ernte- und Wurzelrückständen. Diese Rückstände variieren in ihrer stofflichen Zusammensetzung und damit auch in der Freisetzungsgeschwindigkeit der pflanzenverfügbaren Nährstoffe (BAEUMER, 1992). Die N-Mineralisation aus den Ernterückständen stellt somit einen zentralen Vorfruchteinfluss dar, der sowohl für die N-Versorgung einer nachfolgenden Hauptfrucht als auch für die Sickerwasserbelastung mit Nitrat von Bedeutung ist (KAUL, 1998). Die Geschwindigkeit der N-Mineralisation einer eingearbeiteten Vorfrucht hängt von deren C/N-Verhältnis ab (WEBB et al. 1997). Falls der freigesetzte Stickstoff nicht mit den Niederschlägen ausgewaschen wurde, kann er von der Nachfrucht aufgenommen und in Pflanzenmasse umgesetzt werden (BAEUMER, 1992). Ob der

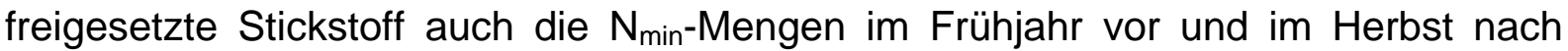
Mais beeinflusst, wird im Folgenden diskutiert.

Die Frühjahrs $\mathrm{N}_{\min }$-Mengen in der Datenkategorie 1 liegen bis auf die Vorfrucht Triticale auf gleichem Niveau. Nach dem Anbau von Triticale folgt aber immer eine Zwischenfrucht, so dass der Einfluss auf die $\mathrm{N}_{\min }$-Menge nicht eindeutig zugeordnet werden kann. Bei den anderen Vorfrüchten ist keine eindeutige Tendenz auszumachen, ob eine Zwischenfrucht nach der Vorfrucht angebaut worden ist oder nicht. Die vorgefundenen $\mathrm{N}_{\min }$-Mengen unterscheiden sich kaum. Die brachliegenden Flächen nach der Vorfrucht Mais weisen, im Gegensatz zu den mit einer Zwischenfrucht bestellten Flächen, keine signifikanten Unterschiede in den $\mathrm{N}_{\min }{ }^{-}$ Mengen im Frühjahr auf. So scheint der Maisanbau in Selbstfolge nicht generell mit hohen $\mathrm{N}_{\min }$-Mengen nach dem Winter einherzugehen.

In der Datenkategorie 2 sind die Frühjahrs $\mathrm{N}_{\min }$-Mengen nach der Vorfrucht Raps vor dem Anbau von Mais tendenziell höher als beim Anbau von Getreide, Hackfrüchten, Zuckerrüben und Mais, welche nahezu auf gleicher Höhe liegen. Auf sechs der dargestellten Winterrapsflächen stand nach dem Rapsanbau keine Zwischenfrucht, diese sechs Flächen zeigen die gleiche Tendenz zu höheren $\mathrm{N}_{\min }$-Mengen im Frühjahr. Speziell der Raps zeigt auch in den Frühjahrs $\mathrm{N}_{\min }$-Gehalten von HEGE et 


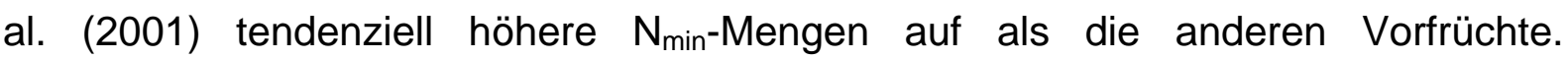
Insbesondere der Winterraps hinterlässt in den Ernterückständen hohe Mengen an schnell mineralisierbaren Stickstoff (WILDENHAYN, 1999). Körnerleguminosen und Mais lassen gegenüber Getreide und Hackfrüchten nach ihrem Anbau höhere $\mathrm{N}_{\text {min- }}$ Mengen im Herbst zurück (TIMMERMANN, 1989). In der Datenkategorie 3 wird der Einfluss der Vorfüchte auf den vorgefundenen $\mathrm{N}_{\min }$-Gehalt im Frühjahr nicht untersucht, da nur von wenigen Flächen die Vorfrucht und der dazugehörige $\mathrm{N}_{\text {min- }}$ Gehalt vorliegen.

Dem Anbau verschiedener Vorfrüchte kann in den Datenkategorien 1 und 2 keinerlei Einfluss auf die Herbst $\mathrm{N}_{\text {min }}$-Mengen nach dem Anbau des Maises nachgewiesen werden. In der Datenkategorie 3 sind bis auf die Vorfucht Weidelgras ebenfalls keine Effekte der Vorfrüchte auf den Herbst $\mathrm{N}_{\min }$-Gehalt nach dem Anbau von Mais in den Datenkategorien festzustellen. Die leicht höheren $\mathrm{N}_{\min }$-Gehalte nach dem Maisanbau beim Weidelgras als Vorfrucht lassen sich möglicher Weise durch den Umbruchtermin des Weidelgrases erklären. Ein zu spätes Umbrechen von Weidelgräsern vor dem Anbau von Mais kann zu erhöhten $\mathrm{N}_{\min }$-Mengen nach der Maisernte führen (RENIUS et al., 1992). Ein etwaiger Düngungseinfluss nach dem Anbau des Winterrapses und vor der Aussaat des Maises kann nahezu ausgeschlossen werden, da diese Feststellung auch auf den ungedüngten Flächen gemacht wurde. Die gedüngten Flächen der Datenkategorie 3 wurden nach dem Winterrapsanbau und vor der Maisausaat im Durchschnitt in sehr geringem Maße mit Stickstoffdünger versorgt. 


\subsection{Einfluss der Witterung auf den Herbst $\mathrm{N}_{\min }$-Gehalt}

Die Witterung vermag die Gefahr der Nitratauswaschung zu beeinflussen (FREDE und DABBERT, 1999). Mit dem Anstieg der Niederschlagsmenge und -häufigkeit nehmen die Sickerwasserbildung und damit die Auswaschungsgefahr von Nährstoffen ins Grundwasser zu (RIEß, 1993). Für die Höhe des $\mathrm{N}_{\text {min }}$-Gehaltes im Herbst ist nach HEYN und SCHAUMBERG (1994) die Niederschlagsmenge von JuliOktober die entscheidende Größe. Dass die Niederschlagshöhe in den Monaten vor der $\mathrm{N}_{\min }$-Beprobung im Herbst den $\mathrm{N}_{\min }$-Gehalt beeinflusst, wird in den Daten aus der Wasserschutzgebietsberatung und in den Erhebungen festgestellt. Die gebildete Niederschlagssumme von Mai bis einschließlich September zeigt schwache negative Korrelationskoeffizienten auf. Dies deutet auf eine Abnahme des vorgefundenen $\mathrm{N}_{\text {min }}$-Gehaltes bei steigenden Niederschlägen hin. Dafür kann es grundsätzlich zwei Erklärungsansätze geben, entweder hat durch den steigenden Niederschlag der Ertrag und damit der Entzug zugenommen oder aber es fanden Auswaschungen statt.

Ein Nährstoffverlust aus landwirtschaftlichen Nutzflächen durch Sickerung während des Sommers wird von SIEMENS et al. (1999) selbst auf Sandböden als unwahrscheinlich eingeschätzt. Auch der Herbst $\mathrm{N}_{\min }$-Gehalt wird nach SCHWEIGERT (1996) kaum durch Auswaschung beeinflusst. Daher könnte diese Korrelation auch durch eine Ertragssteigerung erklärt werden. Dem zu Folge stiege der Ertrag mit zunehmendem Niederschlag und der Mais entzöge dem Boden dadurch mehr Stickstoff. Dieser Erklärungsansatz ließ sich allerdings mittels einer Korrelation zwischen dem Niederschlag und dem Ertrag weder in den Erhebungen noch in den Exaktversuchen nachweisen. Somit ist es wahrscheinlich, dass insbesondere im Mai und Juni, sowie im September und Oktober auf den leichten Standorten der Wasserschutzgebietsberatung Nitratauswaschungen auftraten. STICKSEL et al. (1994a) wiesen in ihren Untersuchungen nach, dass sommerliche Auswaschungen unter Mais auftreten. Auch nach ARNOLD et al. (1993) kann es insbesondere auf leichten Standorten beim Anbau von Silomais während dessen Jugendentwicklung zu Stickstoffverlusten kommen. Gemäß KETELSEN (2001) sind auf leichten Sandstandorten auch Auswaschungsverluste vor der Herbst $\mathrm{N}_{\text {min }^{-}}$ Beprobung möglich. In der Datenkategorie 2 gehen insbesondere auf Böden mit einem Tonanteil von mehr als 25 Prozent höhere Niederschläge mit geringeren $\mathrm{N}_{\text {min- }}{ }^{-}$ 
Mengen einher. Die Klärung der Ursachen scheint mit den erfassten Daten in dieser Kategorie nicht möglich, da die Wasserbindung dieser Böden nach SCHEFFER und SCHACHTSCHABEL (2002) höher als auf Sandböden ist. In der Datenkategorie 3 ist weder bei den gedüngten noch ungedüngten Flächen eine Korrelation feststellbar.

Da nach ANTONOPOULUS (1999) die Stickstoffmineralisierungsrate nicht nur durch den Bodenwassergehalt beeinflusst wird, sondern auch durch die Bodentemperatur, wird im folgenden der Einfluss der Temperatur auf den Herbst $\mathrm{N}_{\min }$-Gehalt diskutiert. Der Mineralstickstoff im Boden besteht größtenteils aus Nitrat, der Rest ist Ammonium, dass bei Temperaturen von mehr als $10^{\circ} \mathrm{C}$ ebenfalls schnell zu Nitrat umgewandelt werden kann (ANTONY et al., 2001). Mit zunehmender Temperatur steigt die N-Mineralisation an (SCHWEIGERT, 1996). Nach MALTZ (2001) besteht zwischen den Herbst $\mathrm{N}_{\text {min }}$-Gehalten und der Temperatur vor der Beprobung eine relativ deutliche Abhängigkeit. In den hier vorliegenden Daten der Kategorie 1 zeigt sich im Hochsommer (Juli, August) eine Zunahme der Herbst $\mathrm{N}_{\min }$-Gehalte bei steigender Temperatur. In der Datenkategorie 2 findet dagegen durchgehend bei einer zunehmenden Temperatur eine Abnahme der Herbst $\mathrm{N}_{\min }$-Gehalte statt. Nach SCHWEIGERT (1996) kann mit zunehmender Temperatur die Nährstoffaufnahme der Pflanzen stärker sein als die Mineralisation. Das würde bedeuten, dass der mineralisierte Stickstoff auf den leichten Böden der Datenkategorie 1 nicht komplett vom Mais aufgenommen werden kann. Hingegen würde der Mais auf den schwereren Böden mehr Stickstoff aufnehmen als durch die Mineralisation freigesetzt worden ist. Die negativen Korrelationskoeffizienten im Mai und Juni der Datenkategorie 1 könnten durch eine schnelle Bestandesetablierung erklärt werden. Eine warme Witterung im Frühjahr fördert nach AUFHAMMER et al. (1991) ein zügiges Auflaufen und den Bestandesschluss, wodurch schnell erhebliche Stickstoffmengen aufgenommen werden. In den Exaktversuchen kann kein Einfluss der Temperatur auf den Herbst $\mathrm{N}_{\text {min }}$-Gehalt mittels der Korrelation nachgewiesen werden.

Da die Temperatur und der Niederschlag gleichzeitig auf das Pflanzenwachstum als auch auf die Mineralisation im Boden wirken, ist die Interpretation der Auswirkungen ohne genaue Bestandes- und Ortskenntnisse sehr schwierig. Daher können in diesem Abschnitt nur mögliche Ursachen aufgezeigt werden. 


\subsection{Vergleich der Aussagekraft von Erhebungsdaten mit Erkenntnissen aus Exaktversuchen}

Ausgangspunkt der Arbeit waren die Thesen, dass

1. die Vereinheitlichung der Rahmenbedingungen im Versuchswesen (ceterius paribus - Prinzip) zur Aufdeckung der vornehmlich produktionstechnisch beeinflussten Haupteffekte im Zuge der Versuchsplanung zur Reduzierung bzw. Dimensionseinengung bei der Ergebnisinterpretation verleiten kann und

2. Erhebungsdaten in solchen Fällen einen größeren Informationsgehalt besitzen, da sie die vielfältigen und sich gegenseitig beeinflussenden Faktoren der Umgebungsmatrix (Umwelt) in sich tragen.

Inwieweit sich diese Thesen durch die Arbeit stützen lassen und welche über den vorliegenden Untersuchungsgegenstand hinausgehenden, verallgemeinerbaren Aussagen zum Erkenntnisgewinn komplexer pflanzenbaulicher bzw. umweltrelevanter Fragestellungen gewonnen werden konnten, soll im Folgenden unter Beachtung methodischer Aspekte diskutiert werden.

Die Arbeit eignet sich für eine derartige Betrachtung in besonderer Weise, da verschiedene Datenquellen unterschiedlicher Datenumfänge und -qualitäten auf die gleiche Fragestellung hin untersucht wurden. Die drei Datenkategorien unterscheiden sich im Umfang und in der Präzision der erhobenen Daten. Während die Datenkategorie 1 aus der Wasserschutzgebietsberatung neben Messdaten auch Schätzwerte beinhaltet, enthält Datenkategorie 2 ausschließlich Messdaten. Beide Datenkategorien wurden auf Praxisschlägen erhoben. In die Datenkategorie 3 fliessen ausschließlich Exaktversuche ein. Insgesamt geht mit zunehmender Datenpräzision eine Reduktion der auswertbaren Datensätze und damit der Umweltinformationen einher.

Bemerkenswerterweise konnten aus allen Datenkategorien, ungeachtet ihrer Unterschiede hinsichtlich Erhebungsumfang und -schärfe, ähnliche Ergebnisse und Zusammenhänge extrahiert werden. Der Einfluss des Gesamt-N und $\mathrm{C}_{\mathrm{t}}$-Gehaltes auf den vorgefundenen Herbst $\mathrm{N}_{\text {min }}$-Gehalt war in allen drei Datenkategorien nachweisbar. Es wurde sogar in den Daten der Wasserschutzgebietsberatung, die 
teilweise auf Schätzungen basierten, ähnliche Abhängigkeiten des Herbst $\mathrm{N}_{\min }$ Gehaltes wie in der Zusammenstellung von Exaktversuchen vorgefunden. Das zeigt, dass sich wesentliche Einflüsse auf die Prüfmerkmale durchaus in Erhebungsdaten niederschlagen und mit den angewandten statistischen Methoden relativ sicher detektierbar sind. Auswertungen von Erhebungsdaten können daher zu einem ähnlichen Erkenntnisgewinn beitragen wie Exaktversuche. Der eindeutige Vorteil in der Untersuchung von Praxisdaten liegt allerdings darüber hinaus in der Möglichkeit, sehr viele unterschiedliche Umweltkonstellationen (Standort, Witterung, Management) in die Untersuchungen einzubeziehen. In strengen faktoriellen Versuchsansätzen auf Parzellenbasis ist es aus Kapazitätsgründen nur in Ausnahmefällen möglich, den Standort in einer hinreichenden Abstufung in das Versuchsdesign aufzunehmen.

Der Nachteil von Erhebungen besteht naturgemäß darin, dass die unadjustierte Datenstruktur eine Auftrennung des die Prüfmerkmale beeinflussenden Umweltkomplexes erheblich erschwert. Kann die Umweltabhängigkeit eines Zusammenhanges (in diesem Fall der Beziehung N-Düngung $\Rightarrow$ Herbst $\mathrm{N}_{\min }$-Gehalt) noch festgestellt werden, ist eine Benennung der konkreten Variationsursache häufig nicht mehr zu bewerkstelligen. Dies hat auch im Zwang zur Variablenaggregation aus Gründen der Vermeidung von Multikollinearität seine Ursachen und ist damit zumindest teilweise ein immanentes Problem der multivariaten statistischen Methoden.

Insofern ist eine sinnvolle Kombination der Auswertung umfangreicher Erhebungen mit dem Instrumentarium des Feldversuchswesens eine empfehlenswerte Strategie des Erkenntnisgewinns. Dies gilt insbesondere für Fragestellungen mit sehr komplexen Wirkungsmechanismen, wie sie im Bereich der Agrar- und Umweltforschung häufig auftreten. Dabei kommt einer übergreifenden Auswertung von Erhebungsdaten eine Doppelrolle zu: Zum einen können praxisrelevante, komplexe Faktorenwirkungen aufgedeckt werden, die mittels orthogonaler Versuchsanstellungen unter kontrollierten Bedingungen näher zu untersuchen und zu beschreiben sind. Ein Beispiel hierfür wäre die in der Faktorenanalyse vorgefundene Komponente, welche die N-Mineralisation näher beschreibt. Hierzu wären weitere gezielte Feldversuche auf unterschiedlichen Standorten mit dem Focus auf das Merkmal der N-Mineralisation sehr zu begrüßen. 
Zum anderen kann die Umweltrobustheit und damit die Verallgemeinerbarkeit von in Einzelversuchen mit spezifischer Umweltmatrix aufgedeckten Zusammenhängen validiert werden. Anhand der gesammelten Daten zeigt sich, dass Aussagen von verschiedenen Versuchsanstellern, wie beispielsweise BÜCHTER (2003) oder LORENZ et al. (1991), nicht generell vor dem Praxishintergrund und auf verschiedenen Standorten haltbar sind.

Diese auf der vorliegenden Datengrundlage getroffenen Ausführungen werden im Übrigen auch durch Literaturangaben bestätigt. So ist es bemerkenswert, dass Autoren, die sich ebenfalls auf Erhebungsdaten stützen (KETELSEN 2001), zu vergleichbaren Ergebnissen gelangten. Auch KETELSEN (2001) war es nicht möglich, eine Korrelation zwischen den vorgefundenen Herbst $\mathrm{N}_{\text {min }}$-Mengen und der $\mathrm{N}$-Düngung bzw. den $\mathrm{N}$-Schlagbilanzen nachzuweisen. Als Ursache für die insgesamt vorgefundene hohe Streuung der Herbst $\mathrm{N}_{\min }$-Gehalte führte KETLESEN (2001) Standorteffekte an. 


\section{Schlussfolgerungen}

Aus dieser Arbeit lassen sich eine Reihe von Schlussfolgerungen und Empfehlungen ableiten, die sowohl die weitere wissenschaftliche Arbeit als auch die konkrete Beratungstätigkeit in den Wasserschutzgebieten betreffen. Die Anregungen werden aus Gründen der Übersichtlichkeit unterteilt in Empfehlungen für die Forschung, die produktionstechnische Beratung sowie in Hinweise für die weitere Erhebung von Daten im Rahmen der Zusatzberatung.

\section{a) Schlussfolgerungen für die Forschung}

- Die wichtigste wissenschaftliche Erkenntnis aus den Daten besteht in der Bestätigung der These, dass die Höhe der Düngung einen geringeren Anteil der N-Überhänge vor Eintritt der Sickerwasserperiode verursacht als bislang angenommen. Es gilt daher für zukünftige Versuchsanstellungen, die Standortparameter intensiver zur Erklärung der zu beobachtenden Prüfmerkmalsausprägung heranzuziehen.

- Bei der Prüfung und zukünftigen Entwicklung von produktionstechnischen Maßnahmen zur Reduzierung der Nitratausträge beim Anbau von Mais, sollte die Wirkung der Maßnahmen auch auf stark mineralisationsfreudigen Standorten getestet werden. Nur hierdurch ist eine klare Aussage zur allgemeinen Wirksamkeit zu erzielen. Sinnvoll wäre es in Zukunft ein Schema zu entwickeln, nach dem der Wasserschutzberater die Wirksamkeit von produktionstechnischen Maßnahmen auf verschiedenen Standorten einschätzen kann.

- Die sehr aufwendige Erfassung und Aufbereitung der meteorologischen Daten (Tageswerte) vermag nicht in dem Maße zur Ursachenklärung beizutragen wie dies erwartet werden durfte. Daraus ist zu schlussfolgern, dass ein hoher Versuchsaufwand zur Erfassung von Witterungsdaten nur dann gerechtfertigt erscheint, wenn er mit der zeitnahen und ebenfalls kontinuierlichen Erfassung von Prüfmerkmalen einhergeht, die in einem engen kausalen Zusammenhang zu den Witterungsparametern stehen. Ansonsten liefern gröbere Modelle eine für die Dateninterpretation hinreichende Information. 


\section{b) Schlussfolgerungen für die produktionstechnische Beratung}

- Trotz der hier festgestellten begrenzten Wirkung der Düngermenge auf die $\mathrm{N}_{\min }$-Menge vor Beginn der Sickerwasserperiode bleiben die Empfehlungen zur Bemessung der Düngung ein zentrales Beratungsinstrument. Das Ausmaß der düngebedingten Zunahme des Herbst $\mathrm{N}_{\min }$-Gehaltes der einzelnen Standorte scheint nicht groß genug zu sein, um die starken Standortunterschiede im Hinblick auf das Grundniveau der Herbst $\mathrm{N}_{\min }{ }^{-}$ Gehalte zu dominieren. Es ergeben sich aus den Daten allerdings Hinweise, die in diesem Zusammenhang größere Beachtung finden sollten. So wird beispielsweise deutlich, dass viele Maisanbauer bei einem stärkeren Einsatz organischer Dünger, der in den Auswertungen nicht per se negativ zu bewerten war, nicht in adäquater Weise mit einer Drosselung der mineralischen Ergänzung reagieren.

- Ein weiterer Aspekt besteht darin, dass auf den mineralisationsfreudigen Böden die rein arithmetische Berücksichtigung des Frühjahrs $\mathrm{N}_{\text {min }}$-Gehaltes (Abzug vom Sollwert) nicht hinreichend ist. Die Umsetzung dieser Erkenntnis in der Beratung wird dadurch erschwert, dass für die Abschätzung der Mineralisationsfreudigkeit eines Standortes derzeit noch keine gleichzeitig verlässlichen wie einfach zu handhabenden Methoden verfügbar sind. Hier ist die Wissenschaft weiter gefordert, praktikable Lösungen zu erarbeiten. Wie die Auswertungen erkennen lassen, eignet sich das C/N-Verhältnis hierzu kaum. Daher erscheint es angebracht, zunächst den Empfehlungen von HUGGER (1992) zu folgen und das langjährige Ertragsverhalten in 0Düngefenstern zur Einschätzung heranzuziehen. Die pragmatische Kategorisierung von Böden nach Bodenart, Humusgehalt und dem Ausmaß organischer Düngung, wie sie VON FISCHER (1993) zur Abschätzung der Mineralisationsneigung vorschlägt, wird durch die vorliegende Untersuchung gestützt, da enge Beziehungen dieser Größen zur erhobenen $\mathrm{N}_{\text {min }}$-Menge feststellbar waren.

- Es lassen sich aus den Daten keinerlei Hinweise dafür ableiten, dass ein Herauslösen des Maises aus der Selbst- in eine Wechselfruchtfolge per se Erfolg versprechend im Sinne einer Reduzierung der $\mathrm{N}_{\min }$-Mengen wäre.

- Die Wirksamkeit von produktionstechnischen Maßnahmen zur Reduktion des Nitrataustrages ist beim Anbau von Mais stark standortabhängig. Daher 
sollten die eingesetzten Maßnahmen auf den Standort und dessen Mineralisationsfreudigkeit abgestimmt werden.

- Höhere Viehbesatzdichten können zu höheren Anteilen von organischer Düngung an der gesamten Düngung führen. Hierbei gilt es zu beachten, dass ein höherer Anteil organischer Düngung nicht per se, sondern nur bei nicht sachgemäßer Anrechnung, schädlich sein kann.

\section{c) Schlussfolgerungen für die weitere Datenerhebung}

- Im Hinblick auf die zukünftige Verbesserung der Nutzung der gewonnenen Daten aus der Wasserschutzgebietsberatung wäre es sinnvoll, eine einheitliche Datenbank zumindest innerhalb eines Bundeslandes aufzubauen. Für diese Datenbank müssten Mindestdatenanforderungen definiert werden, die sowohl die zu erhebenden Merkmale und Kenngrößen als auch deren Aufzeichnung regeln. So sollten neben den Herbst $\mathrm{N}_{\min }$-Gehalten und dem Termin der Probenahme auch Angaben zum Standort und den wichtigsten Bewirtschaftungsdaten enthalten sein (Art, Menge und Termin der Düngung, Erntetermin, mindestens kategoriale Erträge, Zwischenfrucht, Vorfrucht, Viehbesatz etc.). In diesem Zusammenhang wäre es dann auch mit höherer Wahrscheinlichkeit möglich, die Aussageschärfe des Herbst $\mathrm{N}_{\text {min }}$-Gehaltes zu erhöhen.

- Alternativ wäre zu überlegen, ob nicht ein Kernerhebungsbereich geschaffen werden sollte. Innerhalb dieses Bereiches könnte eine begrenzte, aber streng nach Standort- und Betriebskriterien ausgewählte Anzahl an Schlägen konstant über mehrere Jahre präzise beprobt werden. 


\section{Zusammenfassung}

Das Ziel der vorliegenden Arbeit war es, die Bedeutung wichtiger produktionstechnischer und standortbezogener Faktoren sowie deren Wechselwirkungen für die Nitrataustragsgefahr im Maisanbau zu analysieren. Dies sollte durch die Zusammenführung und gemeinsame Verrechnung einer großen Anzahl von Feldversuchen sowie von Daten, die im Rahmen der Wasserschutzgebietsberatung erhoben wurden, erreicht werden.

Dazu wurden drei Datenkategorien gebildet. Kategorie 1 (466 Flächen) repräsentiert Daten aus der Wasserschutzgebietsberatung, die neben Messdaten auch Schätzwerte beinhaltet. Datenkategorie 2 (414 Flächen) stellt ebenfalls Praxiserhebungen dar, deren Merkmalserfassung jedoch ausschließlich auf Messdaten basieren. In die Datenkategorie 3 (137 Flächen) fließen ausschließlich wiederholte Feldversuche ein. Mit zunehmender Datenpräzision ging eine Reduktion der auswertbaren Datensätze sowie der die Erhebungsumwelt beschreibenden Variablen einher. Die Aufteilung der Daten in Kategorien wurde vorgenommen, da von ungleichen Ergebnissen zwischen den Daten aus der Praxis und den Exaktversuchen auszugehen war. Literaturhinweise und der unterschiedliche Kontext der Erhebungen gaben Anlass zu diesem Vorgehen. Des Weiteren wurde erwartet, durch das je nach Datenbestand unterschiedliche Variableninventar die auftretenden Effekte bzw. Interaktionen besser eingrenzen zu können.

Sowohl der Umfang der mit den ökologischen Zielgrößen im mutmaßlichen Zusammenhang stehenden Merkmale, als auch deren vielschichtige gegenseitige Beeinflussung erforderten es, mit multivariaten statistischen Verfahren zu arbeiten.

In allen Datenkategorien konnten, ungeachtet ihrer Unterschiede hinsichtlich Erhebungsumfang und -schärfe, ähnliche Ergebnisse und Zusammenhänge extrahiert werden. Auswertungen von Erhebungsdaten vermögen offenbar zu einem ähnlichen Erkenntnisgewinn beizutragen wie Exaktversuche.

Als ein wesentliches Ergebnis der Untersuchung stellt sich heraus, dass ein genereller, d.h. durch die Umweltmatrix nicht diskriminierter Einfluss der Düngermenge auf den Herbst $\mathrm{N}_{\min }$-Gehalt nicht nachgewiesen werden kann. Vor dem Hintergrund der Vielzahl an einbezogenen Standort- und Umweltbedingungen kann die mineralische Düngung, anders als bei der Betrachtung einzelner Versuchsstandorte, keinen markanten Einfluss auf den vorgefundenen $\mathrm{N}_{\min }$-Gehalt 
im Herbst ausüben. Demnach lässt der $\mathrm{N}_{\min }$-Gehalt im Herbst keine eindeutigen Rückschlüsse auf die vorangegangene Stickstoffdüngung $\mathrm{zu}$, weil in dieser Untersuchung die Standortmerkmale, und hierbei hauptsächlich die des Bodens, das Niveau der Herbst $\mathrm{N}_{\min }$-Gehalte maßgeblich determinieren. Der Herbst $\mathrm{N}_{\min }$-Gehalt lässt sich in dieser Untersuchung am besten durch den Gesamt-N Gehalt, den Kohlenstoffgehalt und den Frühjahrs $\mathrm{N}_{\min }$-Gehalt des Bodens beschreiben. Die aufwendig erhobenen meteorologischen Daten vermochten nicht in der aus der Literatur erwarteten Stärke zur Erklärung der Herbst $\mathrm{N}_{\min }$-Gehalte beitragen. Aufgrund der bekanntermaßen starken Sensitivität der C4-Pflanze Mais gegenüber Temperatureinflüssen wurde dieser Befund nicht erwartet, lässt sich jedoch teilweise mit der äußerst losen und statistisch nicht abzusichernden Beziehung zwischen dem $\mathrm{N}$-Saldo und dem Herbst $\mathrm{N}_{\text {min }}$-Gehalt erklären. Somit reduziert sich der die Höhe des potentiell auswaschungsgefährdeten mineralischen Bodenstickstoffs maßgeblich bestimmende Umweltkomplex zumindest innerhalb der schwerpunktmäßig untersuchten Räume auf die Standortmerkmale.

Die Effekte der düngeunabhängigen produktionstechnischen Maßnahmen auf die Reduzierung des Herbst $\mathrm{N}_{\text {min }}$-Gehaltes ließen sich nicht in gleicher Weise wie die Düngemaßnahmen auswerten, da eine ungleich geringere Anzahl an Datensätzen, zudem noch mit unterschiedlicher Verteilung in den einzelnen Datenkategorien, mit entsprechenden Angaben vorlagen. Dennoch ließen sich innerhalb einzelner Datenherkünfte Tendenzen aufzeigen, welche die positive Bedeutung einzelner Maßnahmen, beispielsweise des Zwischenfruchtbaus, anzeigen. Es erscheint durchaus lohnenswert, bei fortgesetzter gezielter Datensammlung diese Problematik mit einem erweiterten Datensatz weiter zu verfolgen. Ein Einfluss der Viehbesatzdichten auf die Herbst $\mathrm{N}_{\text {min }}$-Gehalte ist hier in keiner der untersuchten Daten nachzuweisen. 


\section{Summary}

\section{Investigation into the variability and causality of the potential for nitrate leaching in the cultivation of Zea mays in Germany}

The objective of this work was to analyse and determine the impact of important management and soil and site-related factors and their interactions on the risk for nitrate leaching following the cultivation of maize. The idea was, to base this study on the analysis of a pool of data from a large number of field trials as well as from data collected by consultants for farmers in water protection areas.

The data were divided into three categories: category 1 (466 fields) represents data from the consultancy in water protection areas, which includes measurements as well as estimates. Category 2 (414 fields) also contains data from farm surveys but all values are measured. Data from scientific field trials with replications belong to category 3 (137 fields). The more precise the data are, the smaller are the data sets, and the smaller is the number of variables that describe the environment from which data were taken.

The reason for dividing the data into categories was that different results were expected from data from farm surveys and scientific experiments. This hypothesis was underpinned by a literature survey and the distinctly different context of the data collection by consultancies, surveys or scientific experiments. Furthermore, it was to be expected that by the different set of variables in each category the effects and interactions could be better isolated and become clearer.

The amount and range of traits that are supposed to be in some context with the ecological criteria and also the complex mutual interference made it necessary to apply multivariate statistical procedures. Despite the differences in data coverage and accuracy between the categories, it was possible to extract similar results and relationships. It seems to be the case that the analysis of data from surveys add similarly to the understanding as do scientifically planned experiments.

One of the main results of the research was that a general influence of the amount of $\mathrm{N}$ fertiliser on the amount of soil mineral nitrogen $\left(\mathrm{N}_{\min }\right)$ in autumn could not be accounted for. Against the background of a multitude of site, soil and environmentrelated factors, the mineral $\mathrm{N}$ fertiliser showed no marked effect on the actual $\mathrm{N}_{\min }$ content in autumn, which is different from the results of an individual experimental 
site. According to this, the $\mathrm{N}_{\min }$ in autumn does not allow to accurately estimate the previous $\mathrm{N}$ fertilisation. This is so as site-related factors and especially the soil conditions significantly determine the level of $N_{\min }$ in autumn. The level of $N_{\min }$ in autumn could best be explained by the total soil $N$, the $C$ content and the $N_{\min }$ in spring.

Contrary to expectations, the extensive survey of meteorological data did not contribute to the elucidation of the autumn $\mathrm{N}_{\min }$ although maize as a C4 plant is expected to be influenced by differences in temperature. This missing effect can, at least partly, be explained by the fact that the relationship between the $\mathrm{N}$ balance and autumn $\mathrm{N}_{\min }$ was found to be very weak. Therefore, it is the more site-related factors that contribute to the differences in the amount of mineral $\mathrm{N}$ prone to leaching, at least for the areas and soils covered in this investigation.

The effects of the more technical factors on the reduction of the autumn $\mathrm{N}_{\min }$ could not be an analysed in the same way as the effect of fertilisation due to insufficient data. There were, nevertheless, within data categories, tendencies towards positive effects of certain measures like, for instance, the cultivation of cover crops. It seems to be promising to pursue the presented investigations further with a continuing and systematic data collection that would lead to an even more extended data set.

The livestock density had no significant effect on the $\mathrm{N}_{\min }$ in autumn in any of the categories. 


\section{Literaturverzeichnis}

Addiscott, T. M., (1996): Measuring and modelling nitrogen leaching: parallel problems. Plant and Soil, $181,1-6$

Albert, E., H. Lippold, (1997): NPK-Bilanzen in langjährigen Dauerversuchen mit differenzierter mineralisch-organischer Düngung. VDLUFA Schriftenreihe 46, Kongressband 1997, 343-346

Albert, E., (2001): Wirkung einer langjährig differenzierten mineralisch-organischen Düngung auf Ertragsleistung, Humusgehalt, Netto-N-Mineralisierung und N-Bilanz. Arch. Acker- Pfl. Boden 46, $187-213$

Amberger, A., (1983): Stickstoffaustrag in Abhängigkeit von Kulturart und Nutzungsintensität im Ackerbau. In: Nitrat ein Problem für unsere Trinkwasserversorgung? Arbeiten der DLG, Band 177, 83-94

Amberger, A., (1989): $\mathrm{NH}_{3}$-Verluste aus der Anwendung organischer und anorganischer Dünger. VDLUFA Schriftenreihe 30, 103-108

Amberger, A., (1996): Pflanzenernährung. 4. Auflage, Ulmer - Verlag

Antonopoulos, V. Z., (1999): Comparison of different models to simulate soil temperature and moisture effects on nitrogen mineralization in the soil. J. Plant Nutr. Soil Sci. 162, 667-675

Antony, F., (1993): Die N-Belastung landwirtschaftlicher Flächen als Grundlage der Quantifizierung und Optimierung des N-Haushaltes von Agrar-Ökotopen. Dissertation, Universität Göttingen

Antony, F., (2001): Praxiseinsatz der Herbst $\mathrm{N}_{\min }$-Methode als Erfolgskontrolle -Anforderungen, Möglichkeiten und Grenzen-. NLFB-Tagung, 26.04.2001, Hannover

Antony, F., C. von Buttlar, L. Fiedler, B. Gödecke, J.Hölscher, A. Löloff, H. Schültken, H. Wacker, (2001): Anwenderhandbuch für die Zusatzberatung Wasserschutz. Niedersächsisches Landesamt für Ökologie, Hildesheim

Appel, T., S. Gronimus, M. Kistner-Othmer, M. Schmücker, (1992): Schlagbezogene N-Bilanzierung in Wasserschutzgebieten unterschiedlicher Bewirtschaftungsintensität. VDLUFA Schriftenreihe 35, 234-237

Appel, T., M. Schmücker, U. Schultheiß, (1993): Möglichkeiten und Grenzen schlagbezogener NBilanzen zur Reduzierung der Nitratbelastung in Wasserschutzgebieten. VDLUFA Schriftenreihe 37, $137-140$

Arnold, U., Ch. Hartmann, H. Goldbach, (1993): Zur Problematik von Grenzwerten: Auswaschung von Nitrat und Ammonium bei Silomais auf einem leichten Sandboden Nordostbayerns. VDLUFA Schriftenreihe 37, 601-604

Aufhammer, W., E. Kübler, H.-W. Becker, (1991): Stickstoffaufnahme und Stickstoffverlagerungspotential unter Maisbeständen. Mais 4/1991, 30-32

Aufhammer, W., E. Kübler, (1997): Einfluss von Reihenweite und Untersaat auf den Silomaisertrag und den $\mathrm{N}_{\text {min }}$-Gehalt im Boden nach der Ernte. Die Bodenkultur, 48 (3), 1997, 151-157

Backhaus, K., B. Erichson, W. Plinke. R. Weiber, (2000): Multivariate Analysemethoden. SpringerVerlag, 9. Auflage

Baeumer, K., (1992): Allgemeiner Pflanzenbau. 3. Auflage, Verlag Eugen Ulmer, Stuttgart

Balik, J., H. W. Olfs, (1998): Einfluss mineralischer und organischer N-Düngung auf Parameter der Bodenfruchtbarkeit in einem 5jährigen Mais-Monokulturversuch. Agribiol. Res. 51 (4), 319-328 
Baumgärtel, G., T. Engels, H. Kuhlmann, (1989): Wie kann man die ordnungsgemäße N-Düngung überprüfen? DLG-Mitteilungen 9, 472-474

Becker, R., K. Schmaler, K. Richter, (1995): Der Einfluss der Bodenbearbeitung beim Anbau winterfester Zwischenfrüchte auf die Ertragsbildung der Folgefrucht Silomais. 39. Jahrestagung der Arbeitsgemeinschaft Grünland und Futterbau, TU München Weihenstephan, 174-178

Becker, K. W., (1999): Zusammenfassender Bericht über den Workshop: $\mathrm{N}_{\min }$-Bestimmungen und deren Anwendung in der Praxis am 23. März in Graz. 8. Gumpensteiner Lysimetertagung, 13. und 14. April 1999, 31-34

Beckmann, T., H. Hasenpusch, M. Kücke, H.J. Altemüller, (1991): Strukturstörungen beim Einbau von keramischen Saugkerzen auf unterschiedlichen Böden. Mitteilungen Dt. Bodenkundl. Gesellsch., 66, I, 107-108

Benke, M., (1992): Untersuchungen zur Nitratauswaschung unter Grünland mittels der SaugkerzenMethode in Abhängigkeit von der Nutzungsart (Schnitt/Weide), der Nutzungshäufigkeit, der Bestandeszusammensetzung (mit/ohne Weißklee) und der Stickstoffdüngung. Dissertation, Universität Kiel.

Benke, M., M. Kayser, B. Thomann, A. Kayser, (1999): Begleitende Untersuchungen des oberflächennahen Sickerwassers mittels der Saugkerzenmethode zur kontinuierlichen Erfassung der Verlagerung und Auswaschung von Nitrat-Stickstoff, Phosphat und Kalium aus landwirtschaftlich genutzten Flächen. Forschungsbericht des Forschungs- und Studienzentrum für Veredelungswirtschaft Weser-Ems der Fakultät für Agrarwissenschaften der Universität Göttingen.

Berg, M., G. Haas, U. Köpke, (1997): Grundwasserschonende Landbewirtschaftung durch organischen im Vergleich zu integrierten und konventionellen Pflanzenbau. VDLUFA Schriftenreihe 46, 615-618

Berger, G., K. Richter, K. Schmaler, (1993): Zur Wirkung des Zwischenfruchtanbaus auf die winterliche $\mathrm{N}_{\text {min }}$-Dynamik im Boden und die N-Konservierungsleistung unter den spezifischen Bedingungen der sandigen Böden des nordostdeutschen Tieflandes. Mitt. Ges. Pflanzenbauwissenschaften, 6, 21-24

Berger, G, K. Schmaler, K. Richter, (1996): N-Aufnahme verschiedener Zwischenfrüchte und ihr Einfluss auf die winterliche $\mathrm{N}_{\min }$-Dynamik sandiger Böden. Arch. Acker- Pfl. Boden., 40, 217-229

Bischoff, W.-A., J. Siemens, M. Kaupenjohann, (1999): Stoffeintrag ins Grundwasser Feldmethodenvergleich unter Berücksichtigung von preferential flow. Wasser und Boden, 51.Jahrg. $12 / 99,37-42$

Blume, H. P., B. Deller, R. Leschber, A. Paetz, S. Schmidt, B. M. Wilke, (2000): Handbuch der Bodenuntersuchung. Beuth Verlag $\mathrm{GmbH}$, Berlin - Wien - Zürich

Bobe, J., M. Wachendorf, M. Büchter, F. Taube, (2003): Vergleich der Nitratkonzentrationen im Grundund Sickerwasser bei variierter Düngungsintensität im Dauergrünland und in einer Maismonokultur Erste Ergebnisse. Mitt. Ges. Pflanzenbauwiss. 15, 190-193

Bosshart, U., (1984): Einfluss der Stickstoffdüngung und der landwirtschaftlichen Bewirtschaftungsweise auf die Nitratauswaschung ins Grundwasser. Dissertation, Gesamthochschule Kassel

Brandhuber, R., Hege, U., (1991a): Maisanbau fördert Nitratauswaschung - besteht der Vorwurf zu Recht? Mais 2/91, 12-14

Brandhuber, R., Hege, U., (1991b): Nitratbelastung des Sickerwassers unter Acker- und Grünland viehhaltender Betriebe - Ergebnisse von Tiefenuntersuchungen. VDLUFA Schriftenreihe 33, $203-208$

Brockerhoff, W., (1994): Düngeplanung mit der N-Bilanzierung? Mais 4/1994, 149-150

Brogowski, Z., K. Dobranski, Konecka-Belten: (1974): The distribution of energetic compounds in mechanical particles of some soils. Polish J. Soil Sci., 2, 99-107 
Brosisus, F., (2002): SPSS 11. MITP Verlag, 1. Auflage

Büchter, M., (2003): Nitratauswaschung unter Grünland und Silomais in Monokultur auf sandigen Böden Norddeutschlands. Dissertation, Universität Kiel

Bühl, A., P. Zöfel, (2002): SPSS 11 - Einführung in die moderne Datenanalyse unter Windows. Pearson Education Deutschland $\mathrm{GmbH}$, 8. Auflage

Capriel, P. (2003): Humusversorgung der Böden. Tagung LfL Agrarökologie, März 2003, Freising

Czeratzki, A., (1973): Die Stickstoffauswaschung in der landwirtschaftlichen Pflanzenproduktion. Landbauforsch. Völkenrode 23, 1-18

Dinnes, D. L., D. L. Karlen, D. B. Jaynes, T. C. Kaspar, J. L. Hatfield, T. S. Colvin, C. A. Cambardella, (2002): Nitrogen management strategies to reduce nitrate leaching in tile-drained midwestern soils. Agron. J. 94, 153-171

Dosch, P., R. Gutser, (1995a): Gasförmige N-Verluste $\left(\mathrm{NH}_{3}, \mathrm{~N}_{2} \mathrm{O}\right)$ unterschiedlich applizierter und aufbereiteter Güllen auf Grünland. VDLUFA Schriftenreihe 40, 717-720

Dosch, P., R. Gutser, (1995b): N2O-Freisetzung nach Applikation von Gülle zu Mais und Grünland. Mitteilungen Dt. Bodenkundl. Gesellsch, 76, 535-538

DVWK, (1990): Gewinnung von Bodenwasserproben mit Hilfe der Saugkerzen-Methode. DVWKMerkblätter 217

Eberdorfer, D., (2002): Gülledüngung zu Körnermais. Mais, 2/2002, 70-71

Eichler, B., B. Zachow, (2002): Beitrag des Zwischenfruchtanbaus zur Reduzierung von Stickstoff- und Phosphatverlusten aus der Landwirtschaft. 46. Jahrestagung, Arbeitsgemeinschaft Grünland und Futterbau, 29. bis 31. August 2002, Rostock, 130-133

Engel, T., A. Mangstl, (1988): Entwicklung des $\mathrm{N}_{\min }$-Gehaltes im Herbst und Winter auf Praxisschlägen. Mitt. Ges. Pflanzenbauwiss. 1, 12-14

Erlenbach, K. H., (1987): Anforderungen an Wasserschutzgebiete - Konsequenzen für die Landwirtschaft. Z. f. Kulturtechnik und Flurbereinigung 28, 161-166

Estler, M., H. Knittel, (1996): Praktische Bodenbearbeitung. Verlags Union Agrar, 2. Auflage

Eulenstein, F., H. Drechsler, K. W. Becker, B. Meyer, (1993): Einfluss des Betriebstyps auf die Nitratverlagerung in mächtigen Lößdecken. Mitt. Ges. Pflanzenbauswiss. 6, 213-216

Feige, W., R. Röthlingshöfer, (1990): Nitratauswaschung aus zwei unterschiedlich bewirtschafteten Ackerböden. Z. f. Kulturtechnik und Landentwicklung 31, 89-95

Franko, U., J. Seeger, T. Schmidt, J. Dreyhaupt, (2001): Einfluss von Standort und Nutzungsintensität auf den N-Haushalt von Ackerböden. 9. Gumpensteiner Lysimetertagung, 24. und 25. April 2001, 33-36

Frede, H.-G., S. Dabbert, (1999): Handbuch zum Gewässerschutz in der Landwirtschaft, Verlag ecomed, 2. Auflage

Fürstenfeld, F., T. Hetterich, (1992): EUF-Bodenuntersuchung und Düngeberatung in einem. Mais 4/1992, 33-34

Gebauer, W. G., H. Schaaf, (1993): Ergebnisse kombinierter organischer und mineralischer NDüngung zu Silomais - Untersuchungsergebnisse im Rahmen der Wasserschutzgebietsberatung im Landkreis Hersfeld-Rotenburg. VDLUFA Schriftenreihe 37, 153-156

Geyer, B., H. Marschner, (1990): Charakterisierung des Stickstoffversorgungsgrades bei Mais mit Hilfe des Nitrat-Schnelltestes. Z. Pflanzenernährung. Bodenk. 153, 341-348 
Gölz-Huwe, H., W. Simon, B. Huwe, R. van der Ploeg, (1989): Zum jahreszeitlichen Nitratgehalt und zur Nitratauswaschung von landwirtschaftlich genutzten Böden in Baden-Württemberg. Z. Pflanzenernähr. Bodenk., 152, 273-280

Goulding, K. W. T., C. P. Webster, (1992): Methods for measuring nitrate leaching. Aspects of Applied Biology, 30, 63-70

Goulding, K., (2000): Nitrate leaching under arable and horticultural land. Soil Use and Management $16,145-151$

Gröblinghoff, F. F., (1992): Strategien umwelt- und bedarfsgerechter Düngung im Anbau von Mais Bodenuntersuchungsabhängiges Verfahren, In: „Zielsetzung und Strategien der integrierten Pflanzenproduktion am Beispiel des Maisanbaues,", Pflanzenbau - Symposium in Soest am 14. und 15. Juli 1992

Gutser, R., K. Vilsmeier, (1988): Mineralisation verschiedener Zwischenfrüchte und N-Verwertung durch Pflanzen. Kali-Briefe 19, H. 3, 199-211

Gutser, R., R. Manhart, (1990): Mulchsaat von Mais bereits praxisreif? Mais 1/1990, 33-36

Gutser, R., B. Wagner, (2000): Gute Noten für den Maisanbau. Mais, 2/2000, 48-51

Häckel, H., (1999): Meteorologie. Eugen Ulmer Verlag, Stuttgart, 4. Auflage

Hahnenkamm, O., G. Kormann, M. Peterrenas, (2000): Magisches Dreieck für Mais. Dlz 4/2000, 20-25

Hamm, A., (1991): Studie über Wirkungen und Qualitätsziele von Nährstoffen in Fließgewässern. Academia - Verlag Sankt Augustin

Hao, X., C. Chang, G. R. Travis, F. Zhang, (2003): Soil carbon and nitrogen response to 25 annual cattle manure applications. J. Plant Nutr. Soil Sci. 166, 239-245

Hartmann, C., (1993): Boden- und kulturspezifische Richtwerte für den Nitratgehalt des Bodens im Herbst - Erfahrungen aus Oberfranken 1989-1992. VDLUFA Schriftenreihe 37, 95-98

Hecht, W., H. Stumpe, J. Garz, (1980): Der Einfluss von langjährigen unterschiedlichen Düngungs- und Anbaumaßnahmen auf das Stickstoffnachlieferungsvermögen von Ackerböden. Arch. Acker- uPflanzenbau u. Bodenk. 24 (9), 593-597

Hege, U., R. Brandhuber, (1990): Nitratbelastung des Sickerwassers bei intensivem Ackerbau. VDLUFA Schriftenreihe 32, 211-216

Hege, U., (1996): Bodenuntersuchung auf $\mathrm{N}_{\min }$. Mais 2/1996, 60-61

Hege, U., E. Kressirer, R. Raupenstrauch, (1998): Nitratgehalte des Sickerwassers - Ergebnisse von Tiefenuntersuchungen. VDLUFA Schriftenreihe 49, 573-576

Hege, U., H. König, K. Offenberger, (2001): Zehn Jahre Stickstoffmonitoring in Bayern. VDLUFA Schriftenreihe 00/2001, 1-8

Herold, L., M. Kerschberger, E. Höpfer, (1996): Beziehungen zwischen N-Bilanz und $\mathrm{N}_{\text {min }}$-Gehalt des Bodens im Herbst. VDLUFA Schriftenreihe 44, 587-590

Hersemann, H., J. Lammel, (1990): Stickstoffbilanzen auf Schlag- und Betriebsebene und deren Konsequenzen für die Stickstoffausträge. VDLUFA Schriftenreihe 32, 283-287

Heyn, J., G. Schaumberg, (1994): 15 Jahre hessisches $\mathrm{N}_{\min }$-Testflächenprogramm - Ergebnisse und Erfahrungen. VDLUFA Schriftenreihe 38, 281-284

Hilmes, G., H. Böckler, J. Ilsemann, U. Müller, R. van der Ploeg, (1998): Abschätzung und Darstellung des Nitratauswaschungsrisikos aus landwirtschaftlich genutzten Böden im Winter am Beispiel von Niedersachsen. Wasser und Boden,50.Jahrg. 10/98, 57-61 
Hoegen, B., W. Werner, (1998): Düngungskonzepte für viehhaltende Betriebe auf Ackerbaustandorten in Nordrhein-Westfalen. Forschungsbericht 58 , Universität Bonn

Horlacher, D., H. Marschner, (1990): Schätzrahmen zur Beurteilung von Ammoniakverlusten nach Ausbringung von Rinderflüssigmist. Z. Pflanzenernähr. Bodenk., 153, 107-115

Horn, D., (1990a): Die Erstellung eines Stickstoffdüngungsmodells für Mais auf Basis von Feldversuchen und der Elektro-Ultrafiltrationsmethode. Dissertation Gießen

Horn, D., (1990b): Stickstoffdüngung nach EUF - umweltbewusst und ökonomisch. Mais 4/1990, 15-17

Hugger, H., (1989): Umweltschonender Maisanbau in Südbaden. Mais 1/1989, 20-21

Hugger, H., (1992): Stickstoffdüngung zu Mais unter Berücksichtigung der Bodennachlieferung. Mais 2/92, $14-16$

Hugger, H., (1995): Ohne Risiko bei der Düngung sparen? Mais 1/1995, 24-25

Hülsbergen, K. J., K. Rauhe, H. Scharf, H. Matthies, (1992): Langjähriger Einfluss kombinierter organisch-mineralischer Düngung auf Ertrag, Humusgehalt und Stickstoffverwertung. Kühn-Arch. 86 (2), 11-24

Iglewicz, B., D. C. Hoaglin (1993): How to detect and handle outliers. American Society for Quality Statistics Division, Vol. 16

Jacobs, G., L. Laurenz, B. Rösing, (1996): Versuchswesen Pflanzenbau. In: Kooperation Landwirtschaft/Wasserwirtschaft im Kreis Borken, Arbeitsbericht 1992-1996

Jacobs, G., (1998): Wieviel Unterfußdünger braucht der Mais?, Mais 2/98, 60-63

Jakob, F., H.-P. Vielemeyer, Podlesak, (1986): Untersuchungen zur Eignung des Nitratgehaltes von Winterweizen zu Diagnose des N-Ernährungszustandes in Feldbeständen. Arch. Acker und Pflanzenbau Bodenk. 30 (10), 607-612

Jarvis, S. C., E. A. Stockdale, M. A. Shepherd, D. S. Powlson, (1996): Nitrogen mineralization in temperate agricultural soils: Process and management. Advances in Agronomy, 57, 187-237

Jokela, W. E., (1992): Nitrogen fertilizer and dairy manure effects on corn yield and soil nitrate. Soil Sci. Soc. Am. J., Vol. 56, January-February, 148-154

Jovanovic, J. N., M. Wachendorf, F. Taube, (1999):Einfluß einer Grasuntersaat auf den TM- und NErtrag von Silomais. 43. Jahrestagung der Arbeitsgemeinschaft Grünland und Futterbau, Bremen, 100-103

Jovanovic, J. N., M. Wachendorf, F. Taube, (2000): Impact of a grass understorey on the performance of silage maize. Proceedings of the $18^{\text {th }}$ General Meeting of the European Grassland Federation, Aalborg, 446-448

Kaul, H. P., (1998): Zur Mineralisation von Stickstoff aus Ernterückständen. Mitt. Ges. Pflanzenbauwiss. 11, 65-66

Ketelsen, $\mathrm{H}$., (2001): Der $\mathrm{N}_{\min }$-Herbstwert als Instrument der Erfolgskontrolle in der Zusatzberatung Wasserschutz. Tagung des NLÖ / NLFB am 26.04.2001 in Hannover

Kirchmann, H, A. Lundvall, (1998): Treatment of solid animal manures: identification of low $\mathrm{NH}_{3}$ emission practices. Nutrient Cycling in Agroecosystems, 51, 65-71

Klasink, A., Steffens, G., (1997): Nährstoffbilanzen in einem Fruchtfolgeversuch ohne und mit organischer Düngung. VDLUFA Schriftenreihe 46, 375-378 
Klinger, S., F. Timmermann, (1988): Zielsetzung, Organisation und Ergebnisse des Vergleichflächenprogramms in Wasserschutzgebieten Baden-Württembergs. VDLUFA Schriftenreihe 28, II, 231-241

Knoblauch, S., D. Roth, I. Pfleger, (1999): Beziehungen zwischen N-Saldo, $\mathrm{N}_{\min }$ im Boden, Nitratgehalt im Sickerwasser und N-Austrag bei unterschiedlichen Ackerstandorten Thüringens. 8. Gumpensteiner Lysimetertagung, 13. und 14. April 1999, 73-76

Kolb, A., (1989): Ermittlung des Düngebedarfs. Deutsche Zuckerüben Zeitung Nr.4, Juni 1989, 12-13

Köhler, W., G. Schachtel, P. Voleske, (1995): Biostatistik. Springer Lehrbuch Verlag, 2. Auflage

Köppen, D., (1997): Bedeutung von schlagbezogenen Nährstoffbilanzen für die Analyse von Bodennutzungssystemen. VDLUFA Schriftenreihe 46, 379-382

Körschens, M., (1992): Einfluss der organischen Substanz auf bodenphysikalische Eigenschaften und N-Freisetzung. Mitt. Ges. Pflanzenbauwiss. 5, 35-38

Körschens, M., (1998a): Erträge, N-Entzüge und Humusdynamik im statischen Düngungsversuch Bad Lauchstädt, 18 Jahre nach Erweiterung der Versuchsfrage. Arch. Acker- Pfl. Boden. 43, 77-92

Körschens, M., (1998b): The importance of soil organic matter for ecological land use systems. Sustainable Agriculture for Food, Energy and Industry, 583-586

Körschens, M., (1999): Richtwerte für Humusgehalte in Ackerböden. VDLUFA Schriftenreihe 52, 131-134

Krämer, R., E. Kümhof, M. Schmidt, (1999): Düngeplanung nach der $\mathrm{N}_{\min }$-Methode - Sinn oder Unsinn? Wasser und Boden, 51 Jahrg. 4/99, 30-35

Krenn, A., E. Klaghofer, M. H. Gerzabek, (2003): Diskussion seitlicher Randeffekte bei Lysimeterexperimenten am Beispiel der Lysimeteranlage Seibersdorf. 10. Gumpensteiner Lysimetertagung, 29. und 30. April 2003, 33-36

Kücke, M., D. H. Yoon, N. Lütke Entrup, (2001): Ermittlung von Auswaschungsprozessen über Matrixund Makro- Porenfluss in unterschiedlichen Anbausystemen. Mitt. Ges. Pflanzenbauwiss. 13, 234

Lamprecht, J., (1999): Biologische Forschung: von der Planung bis zur Publikation. Filander Verlag, Fürth, 1999

Landwirtschaftskammer Weser Ems, (1999): Ergebnisse von Prüfungen und Feldversuchen mit Mais (1999).

Landwirtschaftkammer Westfalen-Lippe, (2003): Die gemessen Trockensubstanzgehalte bei der Ernte von Mais wurden der Homepage: www.lwk-wl.de entnommen.

Lang, H., (1992): Schutz für Boden und Umwelt. Neue Landwirtschaft, H.8, 36-38;

Lanzrath, U., H. E. Goldbach, (2001): Nährstoffmonitoring. 2. Jahresbericht, VDLUFA (Hrsg.), Dezember 2001

Laurenz, L., (1992): Verminderung des Nitrateintrages in die Oberflächengewässer durch Optimierung der N-Düngung zu Mais. In: „Kooperation Landwirtschaft und Wasserwirtschaft der Stevertalsperre”, Dokumentation 1992, Coesfeld

Laurenz, L. (1998): Kostengünstige und umweltgerechte Maisdüngung, Vortrag vom 22.01.1998 in Senden

Leis, A., H. Theuretzbacher-Fritz, G. v. Unold, (2001): Zusammenfassung des Workshops „Lysimeter Anforderungen, Erfahrungen, Technische Konzepte“ vom 25-26. September 2000 in Graz, Österreich.

9. Gumpensteiner Lysimetertagung, 24 und 25. April 2001 
Lochmann, R., R. van der Ploeg, B. Huwe, (1989): Zur Parametrisierung der Stickstoff-Mineralisation in einem Ackerboden unter Feldbedingungen. Z. Pflanzenernähr. Bodenk., 152, 319-324

Lorenz, F., G. Steffens, H. Vetter, (1991): Der Einfluß gestaffelter Schweinegüllegaben und unterschiedlicher Ausbringungszeitpunkte auf Ertrag und Rest-N-Gehalte im Boden. VDLUFA Schriftenreihe 33, 111-116

Lorenz, G., (1992): Gülledüngung mit ergänzenden Mineral-N-Gaben zur Erzielung optimaler Erträge bei niedrigem Nitrataustrag. Göttinger Bodenkundliche Berichte 99, 1-172

Lorenz, F., G. Steffens, (1997): Stickstoffaustrag und Stickstoffbilanz nach Gülledüngung im Lysimeterversuch. VDLUFA Schriftenreihe 46, 595-598

Lütke Entrup, N., P. Zerhusen, (1992): Mais und Umwelt, Verlag Dr. Kovac

Lütke Entrup, N., H. Wieker, G. Stemann, F.F. Gröblinghoff, (1995a): Maisdüngung nach $\mathrm{N}_{\min }$ und Sollwert, Mais 2/95, 65-67

Lütke Entrup, N., N. Brodowski, H.-U. Hensche, D. Kerstin, (1995b): Umweltrelevante Verhaltensmuster der Landwirte - Umsetzungsstrategien und Transferdefizite für den integrierten Pflanzenbau in NRW. Forschungsvorhaben des Fachbereichs Landbau Soest, Universität Gesamthochschule Paderborn, Nr.1

Lütke Entrup, N., F.F. Gröblinghoff, H. Wieker, (1997a): Ertragliche Effizienz und ökologische Relevanz verschiedener N-Düngungsstrategien. In : Umweltgerechter und ertragsorientierter Maisanbau ökologische Situation, produktionstechnische Entwicklungen und Zukunftsperspektiven-, Universität Gesamthochschule Paderborn (Hrsg.), Abteilung Soest, 102-115

Lütke Entrup, N., J. Oehmichen, H. Wieker, F.F. Gröblinghoff, (1997b): Abschlußbericht zum Forschungs- und Entwicklungsvorhaben: Stickstoffmineralisation des Bodens und Stickstoffbedarf im Maisanbau unter Berücksichtigung zeitabhängiger N-Sollwerte und verschiedener Standorte. Universität Gesamthochschule Paderborn, Abteilung Soest

Lütke Entrup, N., (2001): Zwischenfrüchte im umweltgerechten Pflanzenbau. AID 1060/2001

Lütke Entrup, N., (2002): Maisanbau positiv gesehen. Mais 4/2002, 132-137

LWK-WE (Landwirtschaftskammer Weser-Ems), (1997): $\mathrm{N}_{\min }$-Bericht 1997. Eigenverlag

Maidl, F.X. und G. Fischbeck, (1989): Neue Wege einer gezielten Stickstoffdüngung zu Mais. VDLUFA Schriftenreihe 30, Kongressband 1989, 143-148

Maidl, F.X., (1990): Stickstoffverwertung bei Mais. Mais 3/1990, 22-24

Maidl, F.X., (1997): Güllestickstoffwirkung im Ackerbau und Möglichkeiten zur optimierten Düngung im Hackfruchtbau. In: Umweltverträgliche Gülleaufbereitung und Verwertung. KTBL-Arbeitspapier 242, Darmstadt, 203-210

Maidl, F.X., A. Aigner, (1998): Bedeutung von Anbauverfahren und Zwischenfruchtart für NKonservierung und Nitrataustrag. Mitt. Ges. Pflanzenbauwiss. 11, 115-116

Maidl, F.X., E.Sticksel, R. Valta, (1999): Untersuchungen zur verbesserten Gülleverwertung im Maisanbau. 1. Mitteilung: Verwertung von Güllestickstoff durch Silo- und Körnermais bei unterschiedlichen Applikationsverfahren. Pflanzenbauwissenschaften, 3 (1), 1999, 9-16

Maiskomitee, (1999): Die Empfehlung zu den optimalen Trockensubstanzgehalte bei der Ernte von Mais wurden der Homepage: www.maiskomitee.de entnommen.

Maltz, D., (2001): Witterungsbereinigung von $\mathrm{N}_{\min }$-Werten. Tagung des NLÖ / NLFB am 26.04.2001 in Hannover 
Mannheim, T., J. Braschkat, H. Marschner, (1995): Reduktion von Ammoniakemissionen nach Ausbringung von Rinderflüssigmist auf Acker- und Grünlandstandorten: Vergleichende Untersuchungen mit Prallteller, Schleppschlauch und Injektion. Z. Pflanzenernährung und Bodenkunde, 158, 535-542

Martinez, J., G. Guiraud, (1990): A lysimeter study of the effects of a ryegrass catch crop, during a winter wheat/maize rotation, on nitrate leaching and on the following crop. Journal of Soil Science, 41 , 5-16

Merbach, W., A. Wurbs, (1997): Zeitweilige N-Konservierung durch Winterzwischenfrüchte und der Einfluss auf Folgefrüchte und N-Verlagerung. VDLUFA Schriftenreihe 46, 395-398

Merbach, W., A. Wurbs, C. Latus, (1997): Zeitweilige N-Konservierung durch Winterrübsen und ihr Einfluss auf Folgefrüchte und N-Verlagerung im Boden. Mitt. Ges. Pflanzenbauwiss. 10, 187-188

Mohaupt, V., H. Herata, M. Bach, H. Behrendt, (2000): Kläranlage saniert - Woher kommen Gewässerbelastungen heute? Vortrag: „Wasser - Berlin 2000“, 23.-27.10.2000

Müller-Thomson, U., K. Mueller, H. P. Blume, (1998): Bodenkundliche Kartierung und Untersuchungen zur Abschätzung der Nitratbelastung des Grundwassers in den Wasserschutzgebieten der Insel Föhr. Z. f. Kulturtechnik und Landentwicklung 39, 169-174

Müller, J., M. Kayser, T. Schiermann, (2003): Einflüsse düngeunabhängiger Faktoren auf ausgewählte Kenngrößen der Stickstoffverwertung von Zea mays. 47. Jahrestagung der Arbeitsgemeinschaft Grünland und Futterbau, Braunschweig, 275-278

Nemeth, K., (1988): Wissenschaftliche Grundlagen der EUF-Stickstoffempfehlung zu Getreide und Hackfrüchten. 3. Internationales EUF-Symposium Mannheim, Bd. 1, 11-46

Neu, A., (1999): Stickstoff-Immobilisations- und Mineralisierungsprozesse im Boden während der Nachernte-Phase unter besonderer Berücksichtigung der mikrobiellen Biomasse. Dissertation, Universität Bonn

Neubauer, W., R. Kohls, (1994): Ergebnisse zur konservierenden Bodenbearbeitung bei Kartoffeln und Mais unter besonderer Berücksichtigung des Stickstoffs in Pflanze und Boden. Mitt. Ges. Pflanzenbauwissenschaften, 7, 47-50

Nieder, R., J. Richter, (1986): C- und N- Festlegung in Böden Südostniedersachsens nach Krumenvertiefung. Z. Pflanzenernähr. Bodenk. 149, 189-201

Nitsch, A., (1991): Maisanbau in Wasserschutzgebieten muss kein Problem sein. Mais 1/1991, 19-21

Nitsch, A., (1992): N-Düngung nach Nitracheck-Test. In: : „Zielsetzung und Strategien der integrierten Pflanzenproduktion am Beispiel des Maisanbaues,", Pflanzenbau - Symposium in Soest am 14. und 15. Juli 1992

Nitsch, A., (1997): Maisdüngung: Viele Fragen - einfach beantwortet. Mais 2/1997, 60-63

Nitsch, A., (1999):Optimierung der N-Düngung zu Silomais mit dem Nitracheck-Test und Ausblick auf die Grünlanddüngung. 43.Jahrestagung der Arbeitsgemeinschaft Grünland und Futterbau, Bd. 1, 1999, Bremen, 63-67

Olfs, H.W., (1992): Charakterisierung des N-Umsatzes im Boden durch mikrobiologische und chemische Parameter und Bedeutung dieser Kenngrößen für die Ableitung von NDüngebedarfsprognosen. Dissertation Bonn

Olness, A., G. R. Benoit, K. Van Sickle, J. Rinke, (1990): Effect of planting date on time and rate of nitrogen accumulation by maize. J. Agron. Crop. Sci. 164, 42-53

Ortseifen, U., B. Scheffer, (1997): Der Nitrataustrag aus dem durchwurzelten Boden typischer Regionen Niedersachsens. Z. f. Kulturtechnik und Landentwicklung 38, 178-183 
Peretzki, F., (1998): Zwischenfruchtanbau jetzt organisieren. dlz 7/1998, 32-35

Peyker, W., (1998): Eng ist erfolgreich, in praxisnah 1/98, hrsg. von der Saaten - Union

Peyker, W., (2000): 30-40 cm Reihenentfernung - Maß aller Dinge? Innovation 1/2000, Broschüre der Deutschen Saatveredlung (DSV)

Peyker, W., (2004): Mais-Engsaat. Mais 2/2004, 70-71

Pfefferkorn, A., (1993): Zu Auswirkungen einer Humusüberversorgung des Bodens auf Pflanzenertrag und -qualität sowie N-Nachlieferung und Grundwasserbildung. Kühn-Arch. 87 (1), 12-22

Pospeschill, M., (2001): SPSS - Durchführung fortgeschrittener statistischer Verfahren. RRZN-Verlag, 2. Auflage

Presterl, T., G. Seitz, M. Landbeck, (1994): Stickstoffeffizienz und Sortengesundheit. Mais 2/1994, $50-53$

Presterl, T., E. Thiemt, (1999): Nährstoffeffizienz und Ertragssicherheit. Mais 4/1999, 132-134

Puhl, C., (1987): Gibt es grundwassergefährdende Fruchtfolgen? DLG-Mitteilungen 102, 60-62

Renius, W., Lütke Entrup, E., Lütke Entrup, N., (1992): Zwischenfruchtbau zur Futtergewinnung und Gründüngung, DLG - Verlag, Frankfurt (Main)

Richards, I.R., Turner, I.D.S., Wallace, P.A., 1999. Manure and fertilizer contributions to soil mineral nitrogen and the yield of forage maize. Nutrient Cycling in Agroecosystems 55, 175-185.

Richter, C., B. Heiligtag, R. Schmidt, E. Kölsch, (1997): Einfluss unterschiedlicher Düngung auf pH, N, C, und die Gehalte an CAL-extrahierbarem K und P im Boden. Z. Pflanzenernähr. Bodenk. 160, 107-111

Riekmann, C., N. Möbius, U. v. Borstel, (2003): Auswirkungen veränderter Reihenweiten bei Silomais auf Ertrags- und Qualitätsparameter. 47. Jahrestagung der Arbeitsgemeinschaft Grünland und Futterbau, Braunschweig, 225-228

Rieß, F. (1993): Untersuchungen zur Nitratauswaschung nach mineralischer und organischer Düngung von Ackerland und Grünland mittels Saugkerzen-Methode. Dissertation, Technische Universität München

Roth, D., Werner, D. (2000): Handbuch der Bodenkunde - Bewässerung. Ecomed-Verlag, 9. Erg. Lfg. 10/2000, 4

Rottmann, L., (1996): Der Anbau von Mais im System der konservierenden Bodenbearbeitung. Diplomarbeit erstellt an der Gesamthochschule Paderborn, Abteilung Soest

Rouve, G., W. Bogacki, (1987): Verringerung der Nitratbelastung des Grundwassers. Tagung in Würzburg, 9. und 10. April 1987, Landwirtschaftskammer und Fachverband N-Industrie

Rück, F., K. Stahr, (1996): Herbst $\mathrm{N}_{\min }$-Werte als Maß der Nitratauswaschungsgefährdung in Abhängigkeit von Böden und Nutzung. Agribiol. Res. 49, 2-3, 97-112

Sachs, L., (2002): Angewandte Statistik. Springer Verlag, Berlin - Heidelberg

Scharpf, H.-C., U. Wehrmann, (1979): Der Mineralstickstoffgehalt des Bodens als Maßstab für den Stickstoffdüngerbedarf ( $\mathrm{N}_{\min }-$ Methode). Plant and Soil 52, 109-126

Scheffer, B., (1994): Zum Einsatz von Stickstoffdüngern mit Nitrifikationshemmstoff in Wasserschutzgebieten. Wasser, Abwasser, 135, 15-19 
Scheffer, B., R. Bartels, (1998): Kann durch den Einsatz stabilisierter N-Dünger der Nitrataustrag aus einem Sandboden verringert werden? VDLUFA Schriftenreihe 49, Kongressband 1998, 633-636

Scheffer, F., P. Schachtschabel, (2002): Lehrbuch der Bodenkunde. Spektrum Verlag, Heidelberg Berlin

Schilling, G. (2000): Pflanzenernährung und Düngung. Eugen Ulmer Verlag, Stuttgart

Schmidt, J. P. (1992): Kritische Anmerkungen zur Ermittlung von Stoffflüssen mittels Saugkerzen Teil I: Theoretische Aspekte der Bodenlösungsgewinnung. Mitteilungen Dt. Bodenkundl. Gesellsch., 67, 143-146

Schnug, E., (1985): Multivariate statistische Verfahren als Hilfsmittel zur Auswertung mehrfaktorieller Düngungsversuche am Beispiel der Faktorenanalyse. VDLUFA Schriftenreihe 16, Kongressband 1985, 615-624

Schröder, D., G. Hoffmann, N. Weritz, (1986): Bodeneigenschaften in viehhaltenden und viehlosen Betrieben der Niederrheinischen Bucht. VDLUFA Schriftenreihe 20, 455-466

Schröder, J.J., W. van Dijk, W.J.M. de Groot, (1996): Effects of cover crops on the nitrogen fluxes in a silage maize production system. Netherlands Journal of Agricultural Science 44, 293-315

Schröder, J.J., (1999): Effect of split applications of cattle slurry and mineral fertilizer-N on the yield of silage maize in a slurry-based cropping system. Nutrient Cycling In Agroecosystems 53 (3), $209-216$

Schultheiß, U., H. Döhler, M. Schmücker, (1996): Beschränkung der Stickstoffdüngermenge in hessischen Wasserschutzgebieten - Konsequenzen für die Landbewirtschaftung. Mitt. Ges. Pflanzenbauwiss. 9, 189-190

Schweiger, P., (1989): Zum Schutz des Grundwassers. DLG-Mitteilungen 1/1989, 1-4

Schweigert, P., (1996): Der Stickstoffhaushalt des landwirtschaftlich geprägten Wassereinzugsgebietes der Innerste im nordwestlichen Harzvorland bei Hildesheim. Dissertation, Universität Hannover

Seidler, K. P., D. Klotz, (1999): Bestimmung der Sickerwasserfließgeschwindigkeit in Lysimetern Zusammenfassendes Ergebnis des Workshop in Neuherberg, 1998. 8. Gumpensteiner Lysimetertagung, 13. und 14. April 1999, 35-36

Siepen, A., (1998): Zur Eignung des CaCl2-Norg-Gehaltes zur Charakterisierung des Stickstoffnachlieferungspotentials des Bodens und Auswirkungen der zusätzlichen Berücksichtigung dieser Kenngrößen in der $\mathrm{N}_{\text {min }}$-gestützten Düngungsempfehlung zu Mais. Dissertation Bonn

Siemens, J., A. Peine, M. Kaupenjohann, (1999): Sickerung während des Sommers in Sandböden. VDLUFA Schriftenreihe 52, 557-559

Simon, W., B. Huwe, R. R. van der Ploeg, (1988): Die Abschätzung von Nitratausträgen aus landwirtschaftlichen Nutzflächen mit Hilfe von $\mathrm{N}_{\text {min }}$-Daten. Z. Pflanzenernähr. Bodenk., 151, $289-294$

Solansky, S., (1987): Möglichkeiten für eine optimierte, umweltschonende Stickstoffdüngung bei intensiver Viehhaltung. Tagung in Würzburg, 9.-10. April, Landwirtschaftskammer und Fachverband NIndustrie

Springob, G. (2003): C- und N- Freisetzungen bzw. Festlegungen in sandigen Böden Norddeutschlandes nach Nutzungsänderungen. Tagung der Landwirtschaftskammer Weser-Ems, 20.02.2003, Oldenburg

Staufer, W., E. Spiess, (2001): Einfluss unterschiedlicher Fruchtfolgen und nachwachsender Rohstoffe auf die Nitratauswaschung. 9. Gumpensteiner Lysimetertagung, 24. und 25. April 2001, 47-50

Stemann, G., N. Lütke Entrup, (1990): Maisanbau mit Grasuntersaaten. Mais 2/1990, 27-32 
Stemann, G., (1992): Begrünungsmaßnahmen während des Anbaues von Mais zum Boden- und Umweltschutz. In: Zielsetzungen und Strategien der integrierten Pflanzenproduktion am Beispiel des Maisanbaues. Universität-Gesamthochschule Paderborn, Abteilung Soest (Hrsg.), 28-34

Sticksel, E., (1994): Experimentelle Untersuchungen und Modellrechnungen zum boden- und nutzungsbedingten Nitrataustrag in einem Trinkwasserschutzgebiet. Dissertation, Technische Universität München

Sticksel, E., F.-X. Maidl, G. Fischbeck, (1994a): Reduzierung des Nitrataustrages unter Silomais auf Böden hoher N-Austragsgefährdung durch angepasste Düngungsstrategien. Agriobilol. Res. 47,3-4 1994, 324-334

Sticksel, E., F.-X. Maidl, G. Fischbeck, (1994b): Nitratbelastung des Sickerwassers auf extrem auswaschungsgefährdeten Böden in Abhängigkeit von Kultur und Düngungsmaßnahme. Mitt. Ges. Pflanzenbauwiss. 7, 51-54

Sturm, H., K. Isermann, (1987): Standort und Bewirtschaftung sind entscheidend. DLG-Mitt. 2, 74-79

Thomas, G. W., R. L. Blevins, R. E. Phillips, M. A. McMahon, (1973): Effect of a killed sod mulch on nitrate movement and corn yield. Agronomy Journal, 65, 736 - 739

Thorup-Kristensen, K., E. Nielsen, (1998): Modelling and measuring the effect of nitrogen catch crops on the nitrogen supply for succeeding crops. Plant and Soil $203,79-89$

Timmermann, F., (1989): Schutz des Wassers und des Bodens im Rahmen der ordnungsgemäßen Landwirtschaft. VDLUFA Schriftenreihe 30, 23-41

Timmermann, F., (1994): Auswirkungen einer verminderten Stickstoffdüngung im Rahmen von Extensivierungsmaßnahmen auf Ertrag und Nitratauswaschungspotential. VDLUFA Schriftenreihe 38, 155-158

Tollenaar, M., M. Mihajlovic, T. J. Vyn, (1993): Corn growth following cover crops: Influence of cereal cultivar, cereal removal and nitrogen rate. Agron. J. 85, 251-255

Valta, R., (1996): Gezielte N-Düngung zu Mais - ohne Mineraldünger? Mais 2/1996, 52-55

Valta, R., (1999): Optimierung der Flüssigmistverwertung im Maisanbau durch verlustfreie Applikation der Gülle und bedarfsgerechte N-Düngungsmaßnahmen. Dissertation, Technische Universität München.

Van Dijk, W., J. Schröder, L.t.Holte, W.d.Groot, (1997): Binden Zwischenfrüchte ausreichend Stickstoff? Mais 2/1997, 64-67

Verbruggen, I., J. Michels, L. Carlier, E. v. Bockstaele, (1998): Reduced N-fertilization on forage crops; effect on soil nitrate nitrogen after harvest and on yield and quality of the crop. Ecological Aspects of Grassland Management 17. EGF Meeting 1998, 599-603

Vetter, H., G. Steffens, (1986): Wirtschaftseigene Düngung. Verlagsunion Agrar.

Vetter, H., G. Steffens, (1988): Bodenbewirtschaftung und Nitratbelastung des Grundwassers bei einem Wasserwerk in Weser-Ems. Z. f. Kulturtechnik und Flurbereinigung 29, 129-140

Von Fischer, D., (1992): N-Düngung zu Mais selbst bilanzieren. In: Zielsetzung und Strategien der integrierten Pflanzenproduktion am Beispiel des Maisanbaues. Universität Gesamthochschule Paderborn, Abteilung Soest (Hrsg.), 68-73

Von Fischer, D., (1993): Mit der Bilanzierung Geld sparen? Mais 2/93, 74-77

Wantulla, A., (1987): Möglichkeiten zur Verringerung des Nitrataustrags bei Gülleanwendung in einer Silomaismonokultur durch Anbau von Zwischenfrüchten und den Einsatz von Dicyandiamid (DIDIN). Dissertation, Universität Bonn 
Wantulla, A., F. J. Vollmer, W. Kühbauch, (1988): Ganzjährige Bodenwasseruntersuchungen zur Bestimmung von Mineralisation und Nitrataustrag in einem schwach gedüngten Löß-Boden. Z. f. Kulturtechnik und Flurbereinigung 29, 140-147

Webb, J., R. Sylvester-Bradley, F. M. Seeney, (1997): The effects of site and season on the fate of nitrogen residues from root crops grown on sandy soils. Journal of Agricultural Science 128, 445-460

Webster, C. P., M. A. Shepherd, K. W. T. Goulding, E. Lord, (1993): Comparisons of methods for measuring the leaching of mineral nitrogen from arable land. Journal of Soil Science, 44, 49-62

Webster, R., (2001): Statistics to support soil research and their presentation. European Journal of Soil Science, June 2001, 52, 331-340

Weihermüller, L., R. Kasteel, H. Vereecken, (2003): Räumliche Wirkung einer Saugkerze bei der Extraktion von Bodenwasser - Ergebnisse einer Simulation. Mitteilungen Dt. Bodenkundl. Gesellsch., $102,149-150$

Weise, G., J. Pickert, R. Schuppenies, (1994): Praxisversuche zum Einfluss von winterharten und abfrierenden Zwischenfrüchten vor Mais im Vergleich zur Winterbrache auf die N-Dynamik im Boden und den Maisertrag. VDLUFA Schriftenreihe 38, 235-238

Weise, G., P. Scheller, (1997): Untersuchungen zu den Stickstoffüberschüssen und deren Verminderung in Futterbaubetrieben mit Sandböden und Niedermoorgrünland. VDLUFA Schriftenreihe 46, 411-414

Werner, W., H. W. Scherer, D. Drescher, (1985): Untersuchungen über den Einfluss langjähriger Gülledüngung auf N-Fraktion und N-Nachlieferung des Bodens. Z. Acker- und Pflanzenbau, 155, 137-144

Werner, W., (1991): Eignung organischer N-Fraktionen zur Kennzeichnung des NNachlieferungspotentials von Böden. Mitteilungen Dt. Bodenkundl. Gesellsch., 66, Heft 2, 1051-1054

Werner, W., H.-P. Wodsak, (1992): Endbericht des Forschungsvorhabens: Untersuchungen zum organisch gebundenen Stickstoffvorrat und Stickstoffnachlieferungspotentials von Böden des Wassereinzugsgebietes des Rheinlandes. Agrikulturchemisches Institut Bonn

Werner, W., (1995): Nährstoffbilanz im Blickfeld von Landwirtschaft und Umwelt. Fachtagung des Verbandes der Landwirtschaftskammern und des Bundesarbeitskreises Düngung am 29./30. März 1995, Würzburg, 111-128

Wieker, H., N. Lütke Entrup, (1995): Verbesserung der Maisdüngung nach $\mathrm{N}_{\min }$ und Sollwert. VDLUFA Schriftenreihe 40, 993-996

Wiesler, F., W.J. Horst, (1988): Sortentypische Unterschiede in der Nutzung des Stickstoffangebotes bei Mais. Mitt. Ges. Pflanzenbauwissenschaften, 1, 121-122

Wiesler, F., W.J. Horst, (1993): Differences among maize cultivars in the utilization of soil nitrate and the related losses of nitrate through leaching. Plant and Soil 151, 193-203.

Wildenhayn, M., (1999): Effizienz pflanzenbaulicher Maßnahmen zur Verminderung der Nitratproblematik in Wassergewinnungsgebieten. Mitt. Ges. Pflanzenbauwiss. 12, 75-76

Ziegler, A., (1991): Nitrat- und Ertragsentwicklung auf Vergleichsflächen in Wasserschutzgebieten Baden-Württembergs. VDLUFA Schriftenreihe 33, 239-244

Zscheischler, J., M. C. Estler, W. Staudacher, F. Groß, G. Burgstaller, H. Streyl, T. Rechmann,.(1990): Handbuch Mais. Verlagsunion Agrar 


\section{Anhang}

Anhang 1: Häufigkeitsverteilung der Herbst $\mathrm{N}_{\min }$-Gehalte $(\mathrm{kg} \mathrm{N} / \mathrm{ha} / 0-90 \mathrm{~cm})$ in den Haupterhebungsjahren (1996-1999 / Datenkategorie 1)
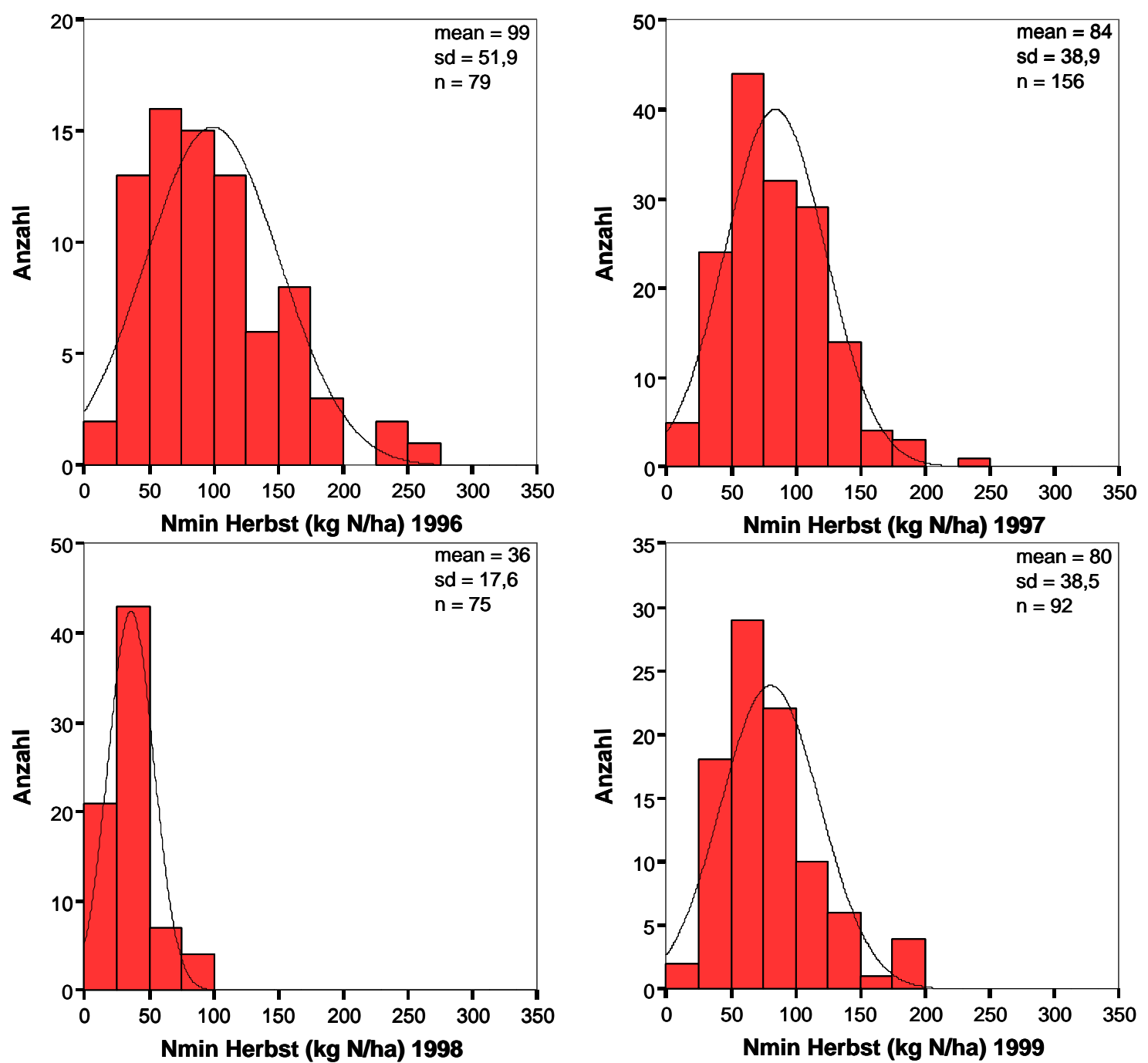
Anhang 2: Häufigkeitsverteilung der Frühjahrs $\mathrm{N}_{\min }$-Gehalte $(0-90 \mathrm{~cm})$ differenziert danach ob sie vor dem 1. Mai oder ab dem 1 Mai gezogen wurden (Datenkategorie 1)

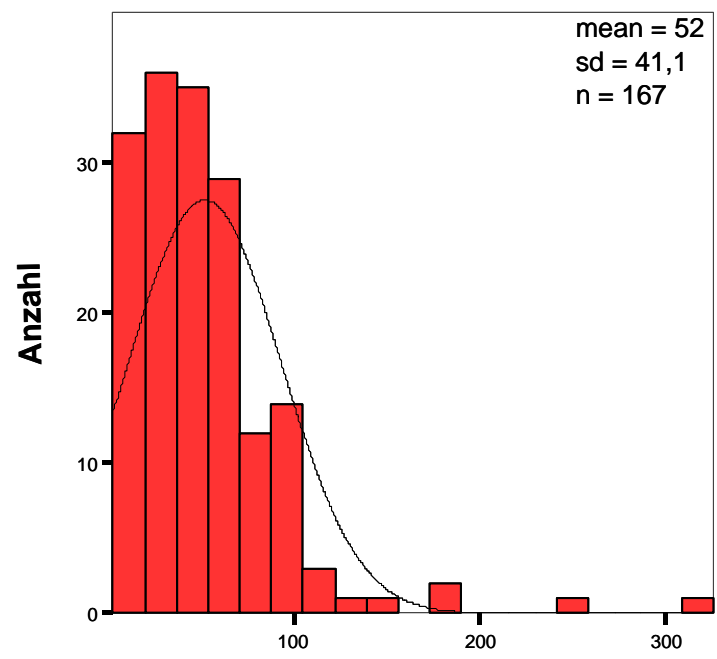

Nmin Frühjahr (kg N/ha/vor 1. Mai)

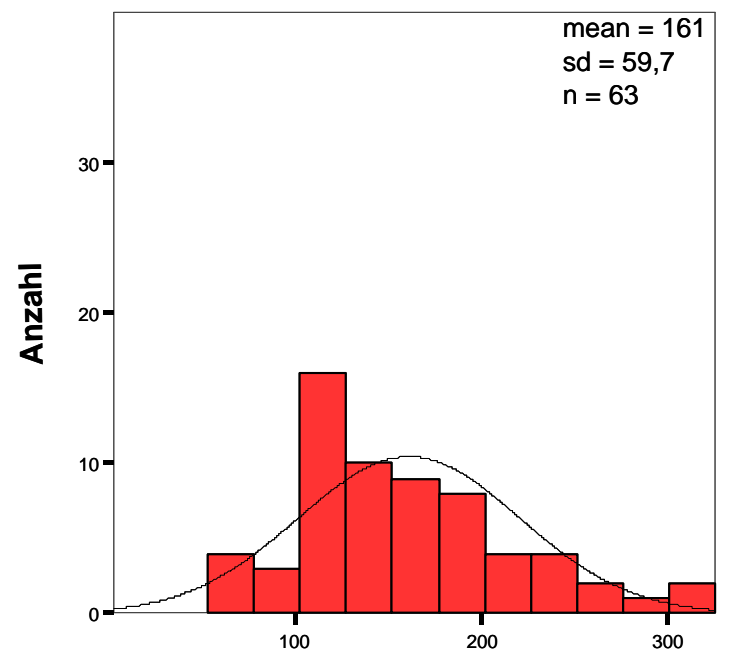

Nmin Frühjahr (kg N/ha/ab 1. Mai)

Anhang 3: Häufigkeitsverteilung der Silo- und Körnermaiserträge (Datenkategorie 1)
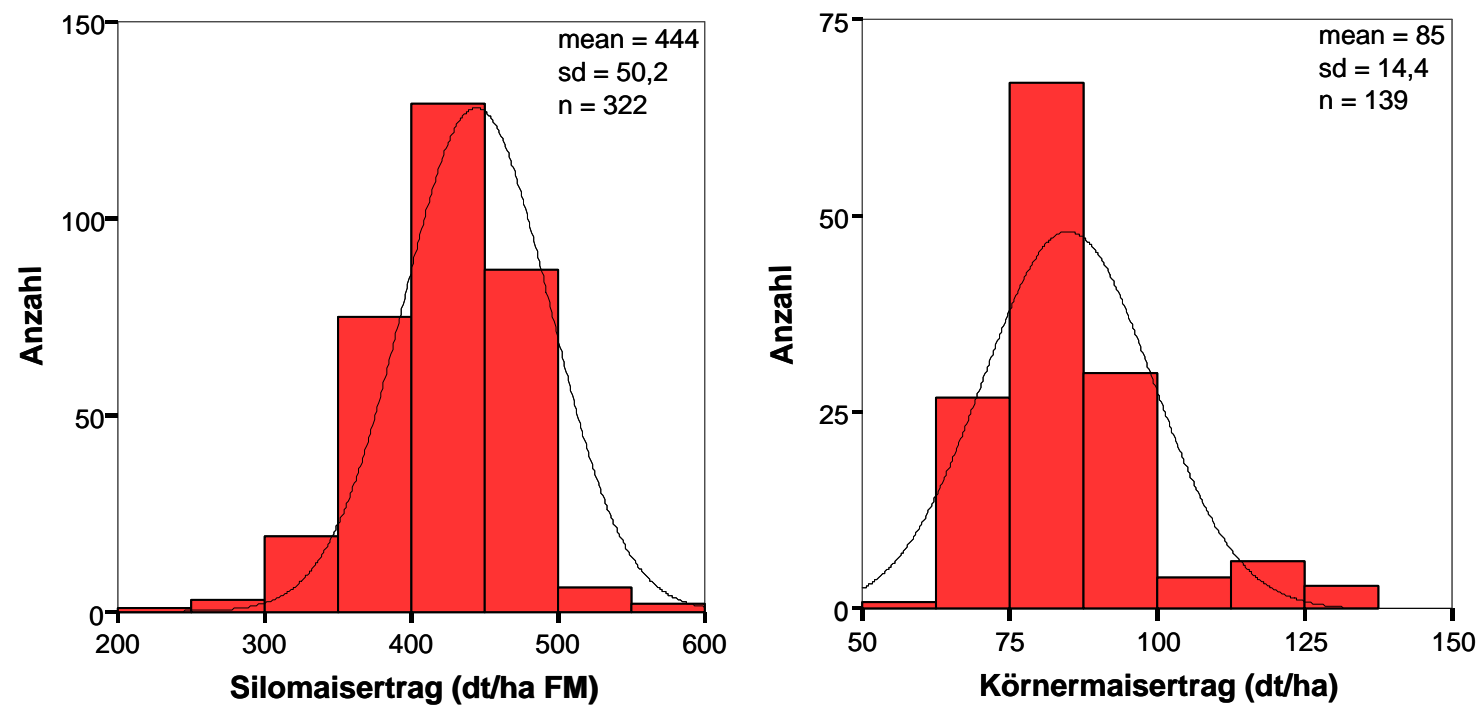
Anhang 4: Häufigkeitsverteilung der Vorfrüchte, getrennt nach den Hauptfrüchten Silo- und Körnermais (Datenkategorie 1)

\begin{tabular}{lcc}
\hline Vorfrucht & Silomais & Körnermais \\
\hline Brache/Stillegung & 2 & 1 \\
Winterweizen & 19 & 22 \\
Winterroggen & 44 & 13 \\
Wintergerste & 79 & 45 \\
Sommergerste & 25 & 8 \\
Hafer & 3 & 1 \\
Triticale & 17 & 15 \\
Winterraps & 3 & 1 \\
Speisekartoffeln & 11 & 6 \\
Feldgras & 3 & 0 \\
Weidelgras/Grünland & 4 & 1 \\
Silomais & 96 & 12 \\
Körnermais & 4 & 9 \\
\hline Gesamtflächen & $\mathbf{3 1 0}$ & $\mathbf{1 3 4}$ \\
\hline
\end{tabular}

Anhang 5: Häufigkeitsverteilung der Zwischenfrüchte, getrennt nach den Hauptfrüchten Silound Körnermais (Datenkategorie 1)

\begin{tabular}{lcc}
\hline Zwischenfrucht & Silomais & Körnermais \\
\hline Brache & 53 & 34 \\
Winterweizen & 1 & 0 \\
Winterroggen & 46 & 5 \\
Winterraps & 21 & 9 \\
Auflaufgetreide & 0 & 2 \\
Ölrettich & 4 & 6 \\
Senf & 117 & 72 \\
Winterrübsen & 2 & 1 \\
Phacelia & 8 & 1 \\
Senf/Raps & 3 & 1 \\
Senf/Ölrettich & 0 & 1 \\
Senf/Raps/Rübsen & 1 & 0 \\
Futterrüben & 6 & 0 \\
Mischung (z.B. Landsberger G.) & 0 & 1 \\
Feldgras & 25 & 2 \\
Weidelgras/Grünland & 3 & 1 \\
Untersaat & 5 & 2 \\
\hline Gesamtflächen & $\mathbf{2 9 5}$ & $\mathbf{1 3 8}$ \\
\hline
\end{tabular}

Anhang 6: Gruppierung der Bodenarten nach leicht, mittel und schwer aus Angaben nach DIN4220 und der Reichsbodenschätzung (LANZRATH und GOLDBACH, 2001)

\begin{tabular}{|c|c|c|c|c|c|}
\hline \multirow{2}{*}{$\begin{array}{l}\text { Ton- } \\
\text { gehalt } \\
\%\end{array}$} & \multirow{2}{*}{$\begin{array}{l}\text { Bodenart- } \\
\text { gruppe }\end{array}$} & \multicolumn{4}{|c|}{ Bezeichnung nach } \\
\hline & & DIN 4220, Te & & Reichsbode & chätzung \\
\hline $\begin{array}{l}<5 \\
5-12\end{array}$ & leicht & $\begin{array}{l}\text { Sand } \\
\text { schw. lehmiger Sand }\end{array}$ & $\begin{array}{l}\text { S } \\
\text { IS }\end{array}$ & $\begin{array}{l}\text { Sand } \\
\text { anlehmiger Sand }\end{array}$ & $\begin{array}{l}\mathrm{S} \\
\mathrm{SI}\end{array}$ \\
\hline $\begin{array}{l}12-17 \\
17-25\end{array}$ & mittel & $\begin{array}{l}\text { stark lehmiger Sand } \\
\text { sandiger Lehm } \\
\text { schluffiger Lehm }\end{array}$ & $\begin{array}{l}\text { St.IS } \\
\text { sL } \\
\text { LL }\end{array}$ & $\begin{array}{l}\text { lehmiger Sand } \\
\text { st. sandiger Lehm } \\
\text { sandiger Lehm }\end{array}$ & $\begin{array}{l}\text { IS } \\
S L \\
S L\end{array}$ \\
\hline $\begin{array}{l}25-45 \\
>45\end{array}$ & schwer & $\begin{array}{l}\text { toniger Lehm } \\
\text { sandig-toniger Lehm } \\
\text { lehmiger Ton } \\
\text { Ton }\end{array}$ & $\begin{array}{l}\mathrm{tL} \\
\text { stL } \\
\text { IT } \\
\mathrm{T}\end{array}$ & $\begin{array}{l}\text { Lehm } \\
\text { schwerer Lehm } \\
\text { Ton }\end{array}$ & $\begin{array}{l}\text { LT } \\
\text { T }\end{array}$ \\
\hline
\end{tabular}


Anhang 7: Durchschnittlicher Temperaturverlauf $\left({ }^{\circ} \mathrm{C}\right)$ in den Haupterhebungsjahren (Datenkategorie 1)

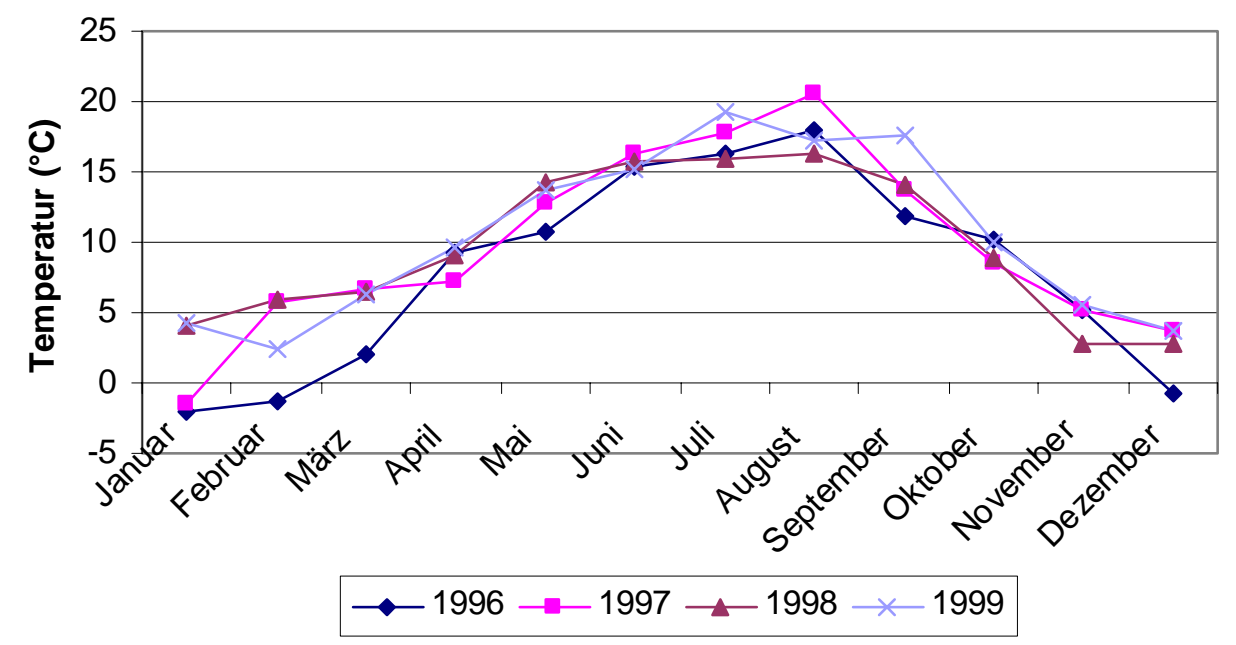

Anhang 8: Durchschnittlicher Niederschlag $(\mathrm{mm})$ in den Haupterhebungsjahren (Datenkategorie 1)

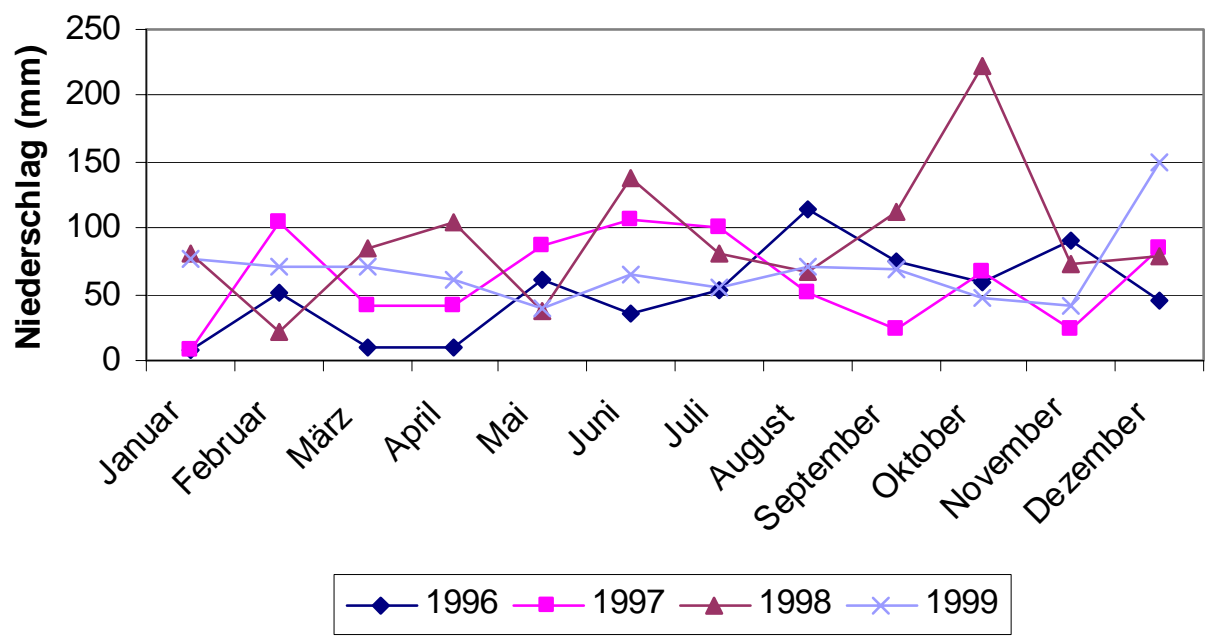


Anhang 9: Durchschnittliche potentielle Evapotranspiration ( $\mathrm{mm}$ ) nach HAUDE in den Haupterhebungsjahren (Datenkategorie 1)

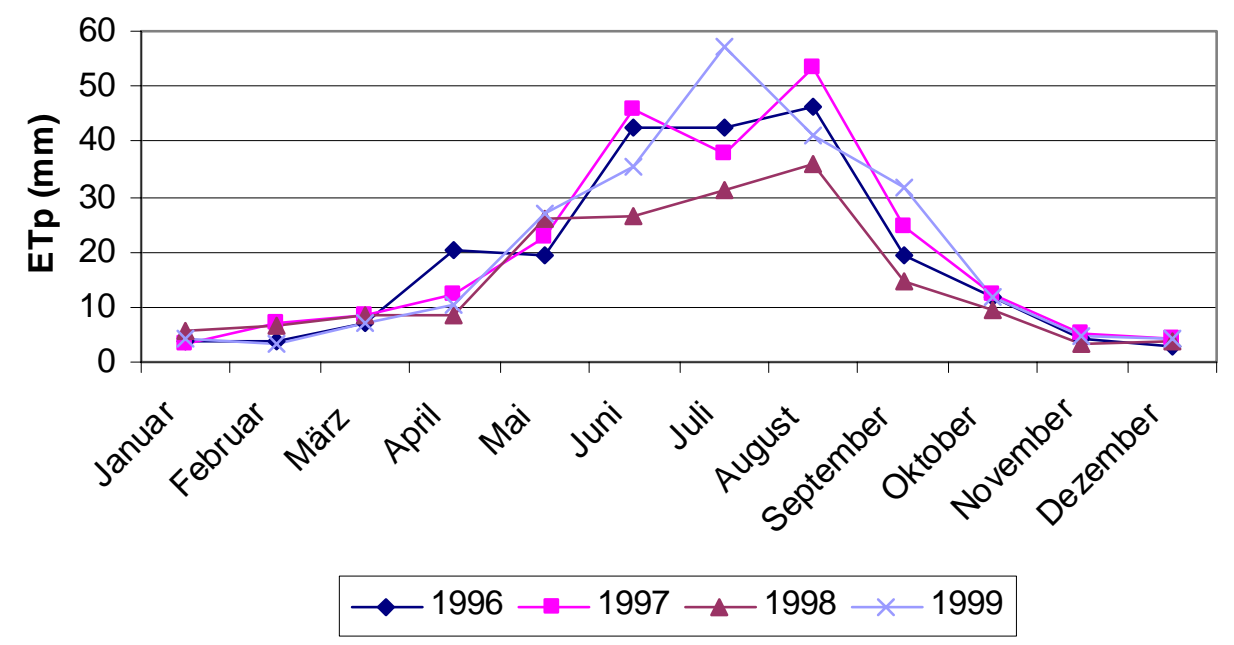


Anhang 10: Häufigkeitsverteilung der aufgewendeten Düngermenge (mineralisch und organisch) in $\mathrm{kg} \mathrm{N} / \mathrm{ha}$. Das erste Diagramm (oben links) beschreibt alle Daten der Datenkategorie 2. Das Zweite (oben rechts), das Dritte (unten links) und das vierte Diagramm (unten rechts) beschreiben die Häufigkeitsverteilungen der Düngermenge je nach Versuchsansteller. (Die Versuchsansteller werden in der X-Achsen Bezeichnung genannt I Datenkategorie 2)
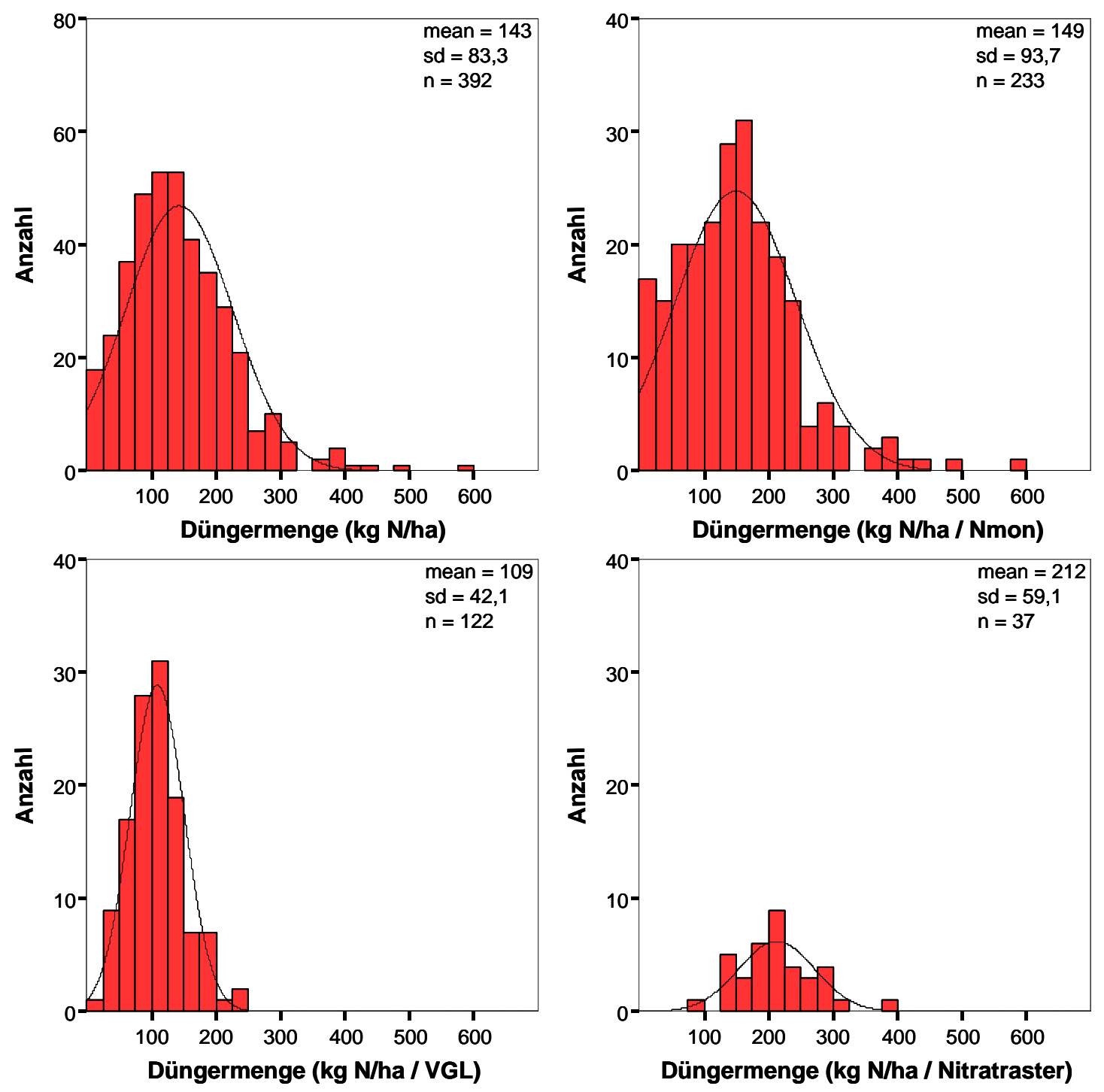
Anhang 11: Häufigkeitsverteilung der Silomaiserträge (links Frischmasse (FM)/ rechts Trockenmasse (TM) / Datenkategorie 2)
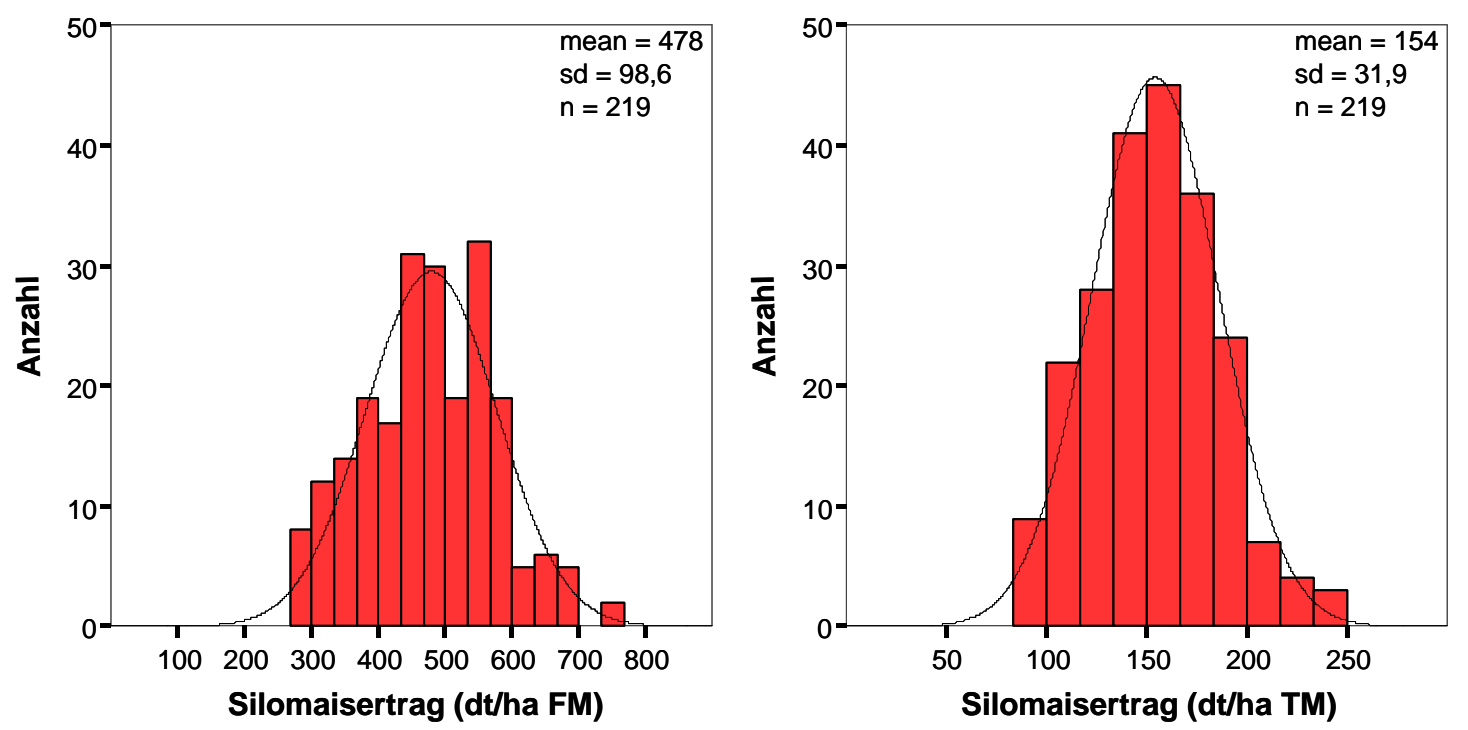

Anhang 12: Häufigkeitsverteilung der Körnermaiserträge (links Frischmasse (FM)/ rechts Trockenmasse (TM) I Datenkategorie 2)
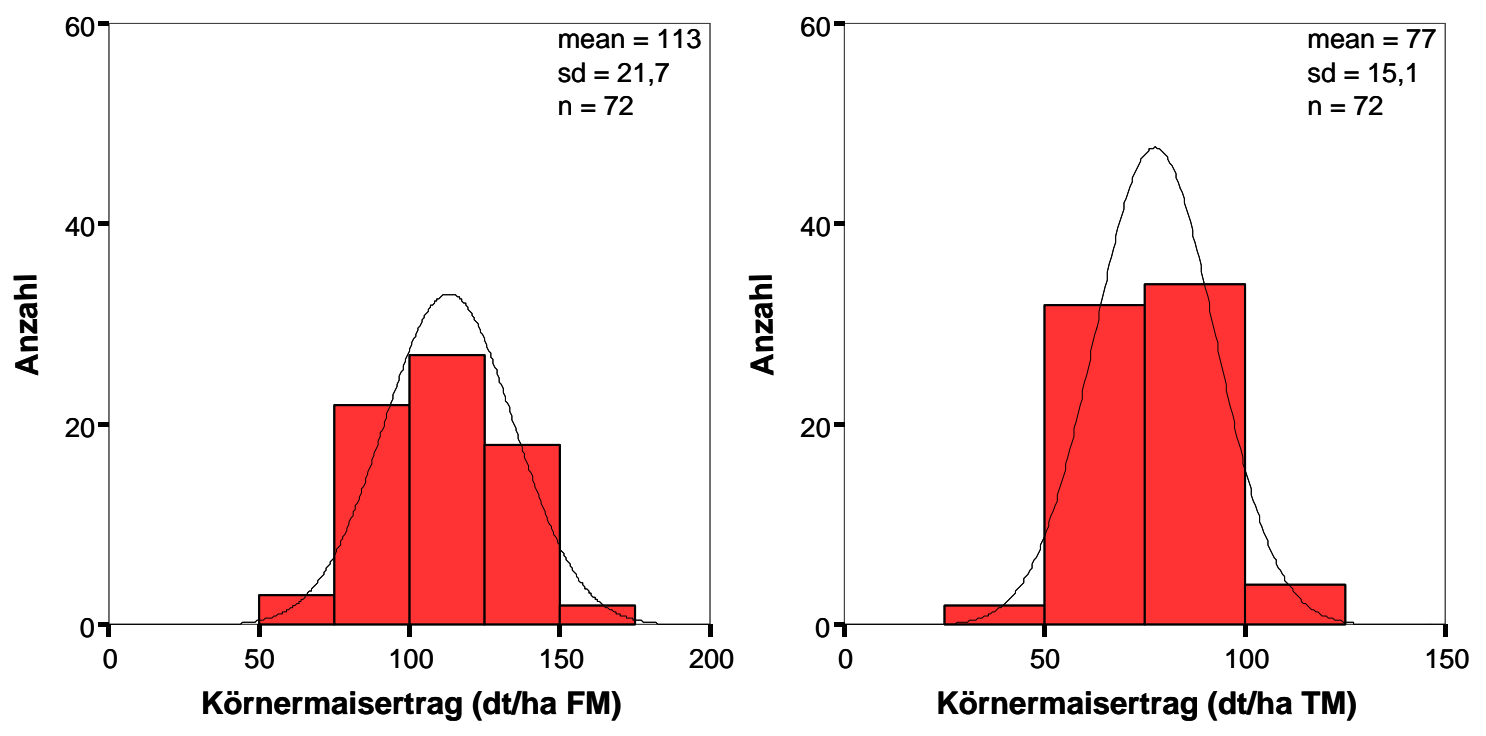
Anhang 13: Häufigkeitsverteilung der Vorfrüchte, getrennt nach den Hauptfrüchten Silo- und Körnermais (Datenkategorie 2)

\begin{tabular}{lcc}
\hline Vorfrucht & Silomais & Körnermais \\
\hline Brache / Stillegung & 2 & 2 \\
Winterweizen & 65 & 34 \\
Sommerweizen & 3 & 0 \\
Winterroggen & 10 & 5 \\
Wintergerste & 55 & 14 \\
Sommergerste & 42 & 9 \\
Hafer & 5 & 2 \\
Getreidegemenge & 2 & 0 \\
Durum & 0 & 1 \\
Triticale & 14 & 2 \\
Grassamen & 1 & 0 \\
Winterraps & 9 & 0 \\
Sonnenblumen & 0 & 1 \\
Öllein & 1 & 0 \\
Hackfrucht & 9 & 0 \\
Zuckerrüben & 9 & 3 \\
Frühkartoffeln & 0 & 1 \\
Speisekartoffeln & 2 & 3 \\
Feldfutter & 2 & 0 \\
Klee & 0 & 1 \\
Kleegras (<50\% Klee) & 3 & 0 \\
Feldgras & 4 & 0 \\
Körnerleguminosen & 2 & 0 \\
Körnererbsen & 1 & 1 \\
Ackerbohnen & 1 & 0 \\
Silomais & 42 & 7 \\
Körnermais & 1 & 12 \\
Gemüse & 1 & 0 \\
Erbsen & 2 & 0 \\
sonstige & 1 & 0 \\
\hline & &
\end{tabular}

Anhang 14: Häufigkeitsverteilung der Zwischenfrüchte, getrennt nach Silo- und Körnermais (Datenkategorie 2)

\begin{tabular}{lll}
\hline Zwischenfrucht & Silomais & Körnermais \\
\hline Keine Zwischenfrucht & 159 & 39 \\
Nicht Leguminose & 93 & 43 \\
Leguminose & 1 & 0 \\
\hline
\end{tabular}


Anhang 15: Häufigkeitsverteilung der Frühjahrs $\mathrm{N}_{\min }$-Gehalte $(0-90 \mathrm{~cm})$ differenziert danach ob sie vor dem 1. Mai oder ab dem 1 Mai gezogen wurden (Datenkategorie 3)
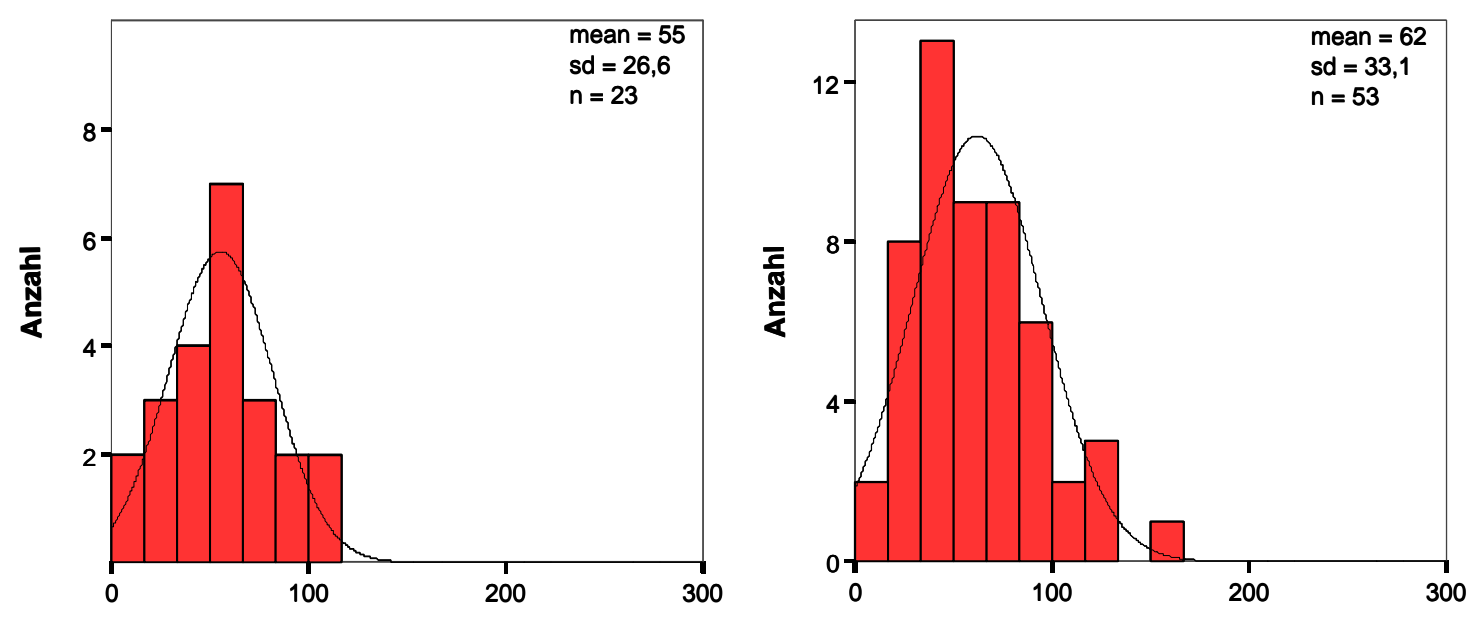

Nmin Frühjahr (kg N/ha/vor 1. Mai) (ohne Düngung)
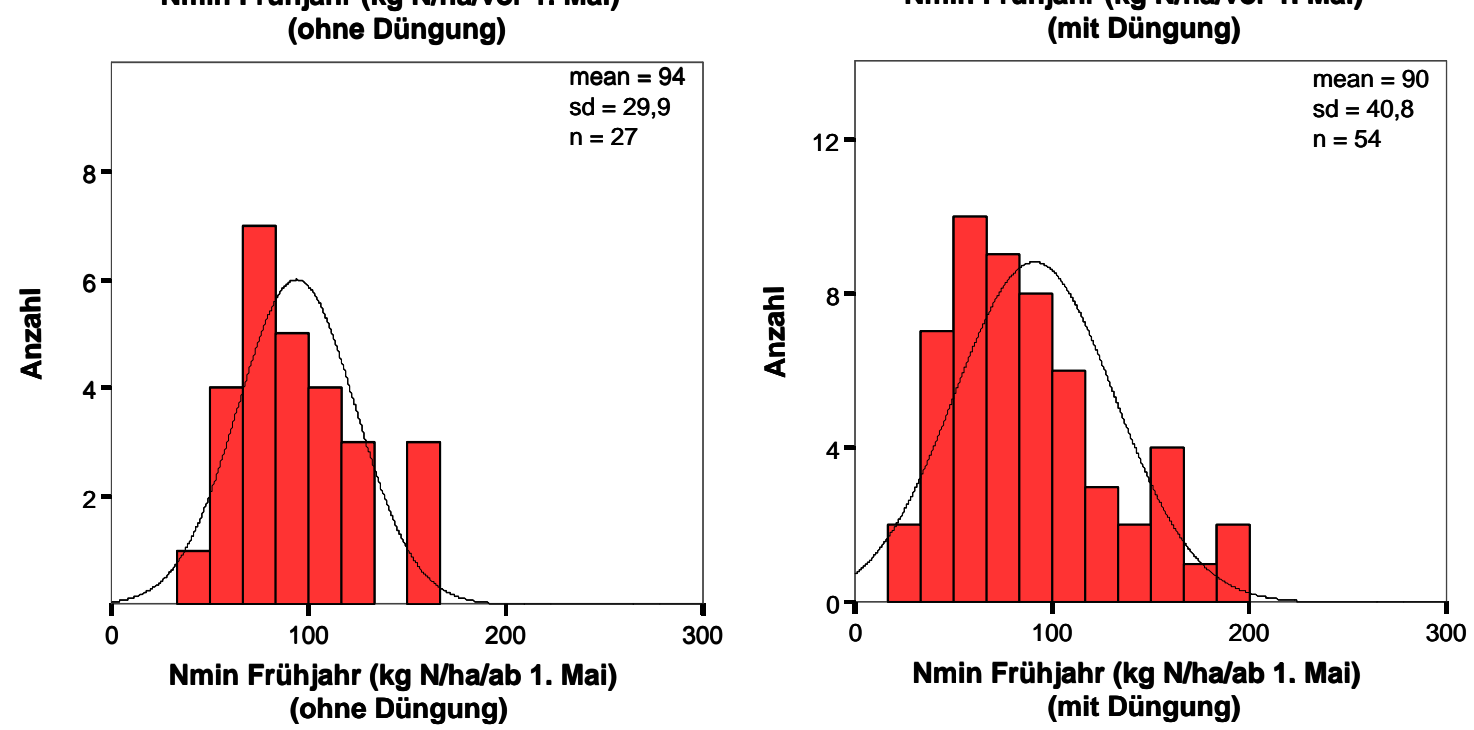
Anhang 16: Häufigkeitsverteilung der Silomaiserträge (Datenkategorie 3)
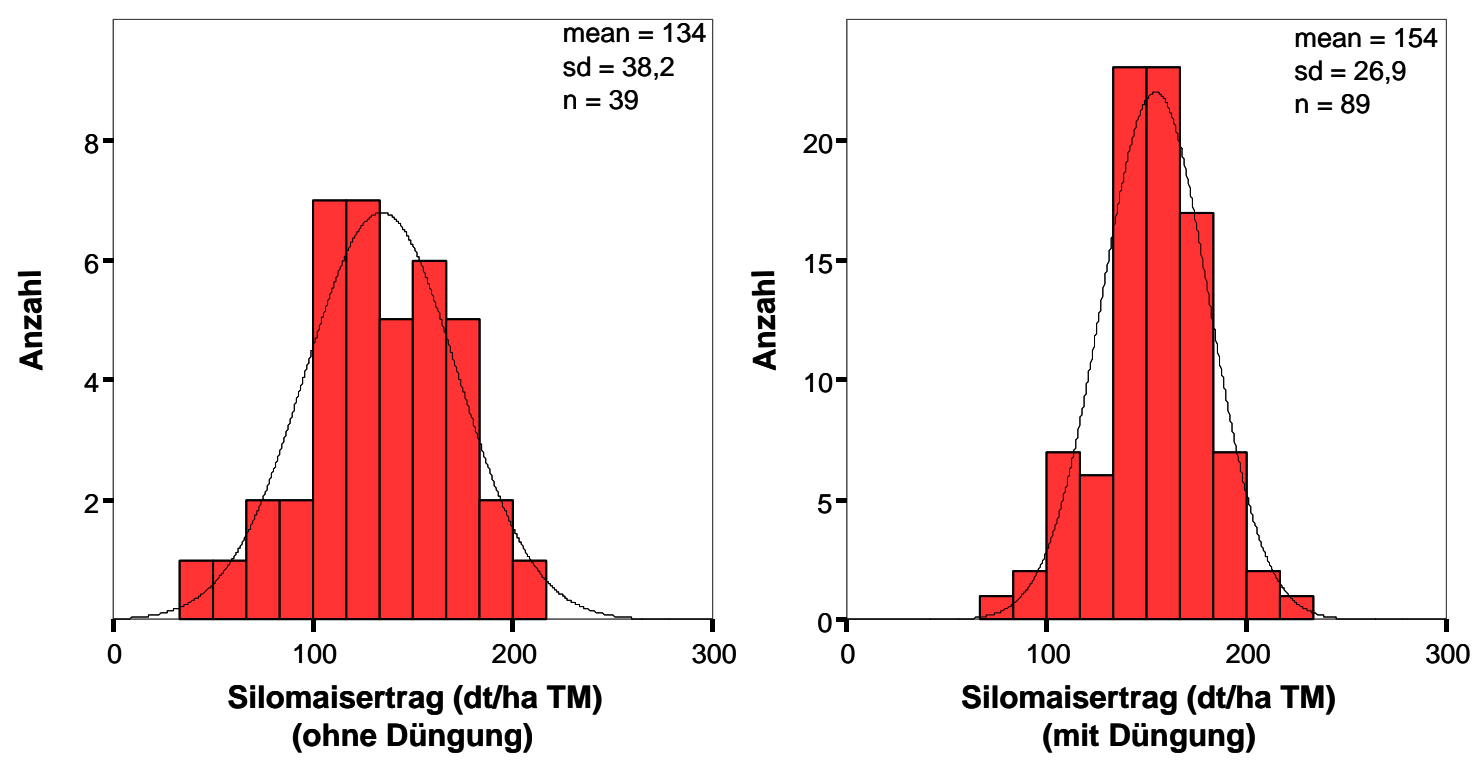

Anhang 17: Häufigkeitsverteilung der Körnermaiserträge (Datenkategorie 3)
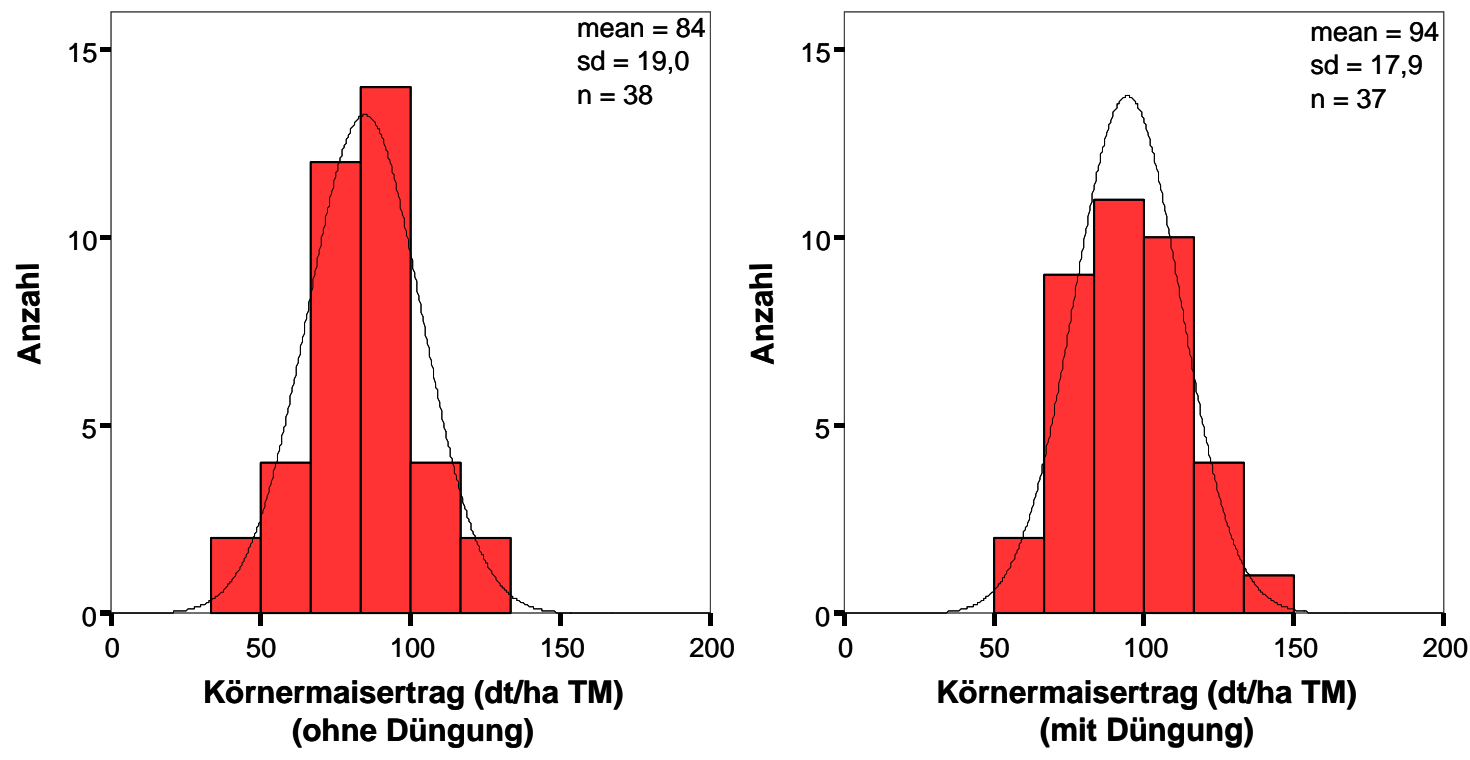
Anhang 18: Häufigkeitsverteilung der Vorfrüchte (Datenkategorie 3)

\begin{tabular}{lcc}
\hline Vorfrucht & Ohne Düngung & Mit Düngung \\
\hline Winterweizen & 10 & 16 \\
Winterroggen & 3 & 3 \\
Wintergerste & 16 & 26 \\
Sommergerste & 0 & 2 \\
Hafer & 0 & 1 \\
Triticale & 7 & 11 \\
Winterraps & 2 & 3 \\
Senf & 1 & 2 \\
Kartoffeln & 4 & 6 \\
Feldfutter & 0 & 1 \\
Kleegras & 1 & 3 \\
Feldgras & 0 & 1 \\
Weidelgras & 6 & 10 \\
Silomais & 20 & 24 \\
Körnermais & 4 & 6 \\
Gemüse & 1 & 1 \\
Sonderkultur & 1 & 1 \\
\hline
\end{tabular}

Anhang 19: Häufigkeitsverteilung der Zwischenfrüchte (Datenkategorie 3)

\begin{tabular}{lcc}
\hline Zwischenfrucht & Ohne Düngung & Mit Düngung \\
\hline Brache & 13 & 33 \\
Winterroggen & 0 & 2 \\
Winterraps & 4 & 6 \\
Senf & 1 & 12 \\
Phacelia & 0 & 1 \\
Hackfrucht & 2 & 3 \\
Weidelgras & 0 & 4 \\
Untersaat & 0 & 1 \\
\hline
\end{tabular}

Anhang 20: Aufteilung der eingesetzten Düngemittel

\begin{tabular}{lccc}
\hline & Wasserschutzgebietsdaten & Erhebungsdaten & $\begin{array}{c}\text { Exaktversuche } \\
\text { (mit Düngung) }\end{array}$ \\
\hline $\begin{array}{l}\text { rein mineralisch } \\
\text { rein organisch }\end{array}$ & 53 & 160 & 110 \\
keine Düngung & 16 & 25 & 1 \\
kombiniert & 0 & 39 & 3 \\
(min. + org.) & 431 & 190 & 21 \\
\hline
\end{tabular}




\section{Danksagung}

Herrn Prof. Dr. J. Isselstein danke ich für die Überlassung des interessanten Themas, für seine Diskussionsbereitschaft und den daraus resultierenden Hinweisen und Ratschlägen zur Fertigstellung dieser Arbeit.

Ich danke Herrn Prof. H. Van den Weghe für die Übernahme des Korreferates.

Dr. J. Müller und Dr. P. Hugenroth danke ich für die fachliche und verwaltungstechnische Betreuung des Projektes.

Den Mitarbeitern des Forschungs- und Studienzentrum für Veredelungswirtschaft Weser-Ems für Ihre permanente Unterstützung und Hilfsbereitschaft.

Mein ganz besonderer Dank gilt den Leuten die diese Arbeit durch die Bereitstellung Ihrer Daten erst ermöglicht haben.

Der Deutschen Bundesstiftung Umwelt für die Finanzierung dieses Forschungsprojektes durch Ihr Stipendienprogramm.

Meinen Eltern und Silke für die Unterstützung während der gesamten Zeit, die während der Arbeit an Werk-, Sonn- und Feiertagen des Öfteren auf mich verzichten mussten. 


\section{Lebenslauf}

$\begin{array}{ll}\text { Name } & \text { Schiermann } \\ \text { Vorname } & \text { Thorsten } \\ \text { Geburtsdatum } & 15.05 .1977 \\ \text { Geburtsort } & \text { Steinfurt (Borghorst) } \\ \text { Familienstand } & \text { ledig }\end{array}$

\section{Schulausbildung}

1983-1987

Grundschule, Neuenkirchen

1987-1993

Gymnasium, Neuenkirchen

1993-1995

Fachoberschule für Agrarwirtschaft, Münster

\section{Studium}

1995-1998

Gesamthochschule Paderborn,

Fachbereich Agrarwirtschaft, Soest

Abschluss: Diplom-Ingenieur (FH)

1998-2000

Georg-August-Universität Göttingen,

Fachbereich Agrarwissenschaft, Göttingen

Abschluss: Master of Science (M. Sc.)

$2000-2004$

Georg-August-Universität Göttingen,

Promotion am Forschungs- und Studienzentrum für

Veredelungswirtschaft Weser-Ems, Vechta

\section{Praktika}

1993-1994

Landwirtschaftliches Praktikum, Greven

\section{Berufstätigkeit}

seit Juli 2004

Angestellter der Firma Krone, Spelle 